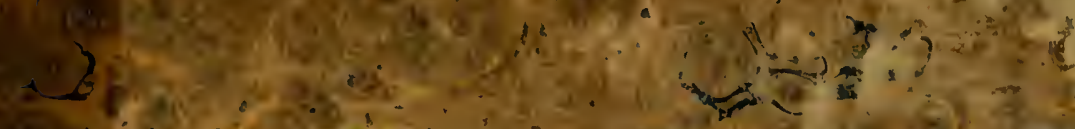
$\theta_{\infty}$

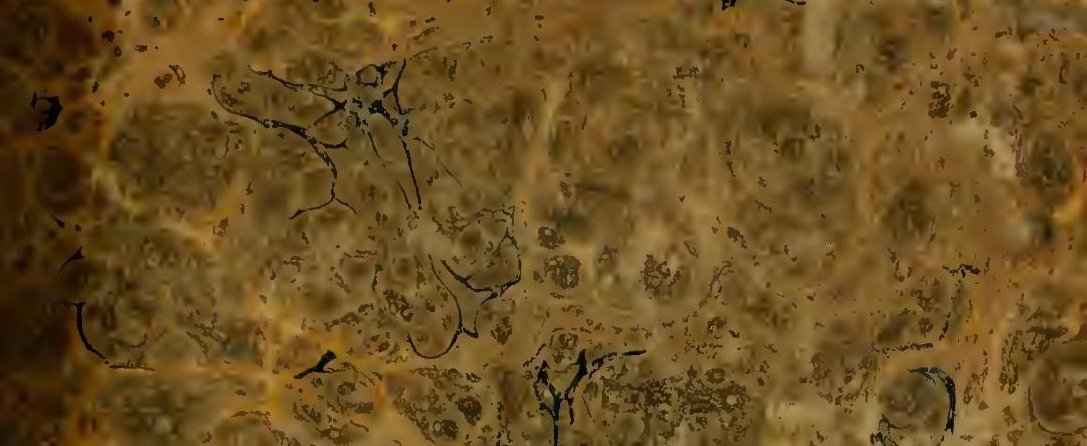

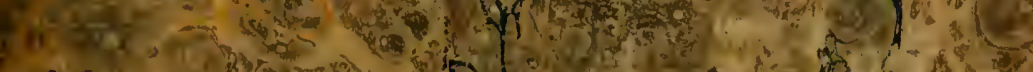

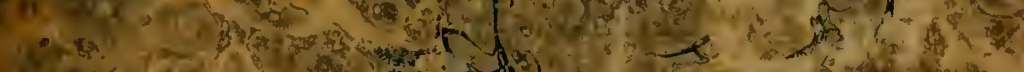

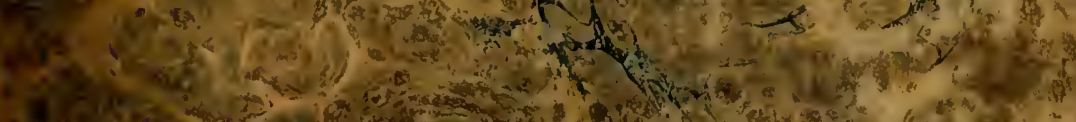

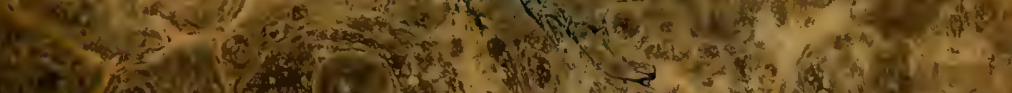

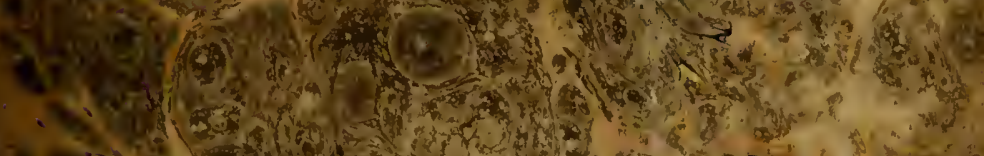

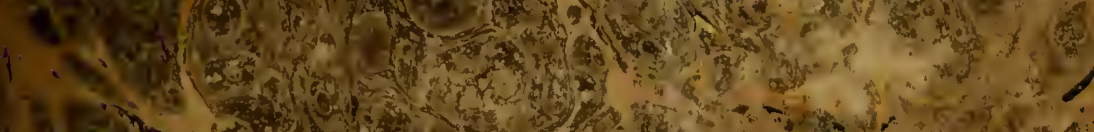
2.

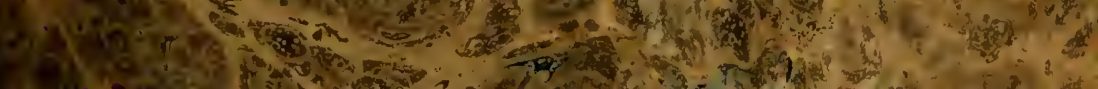

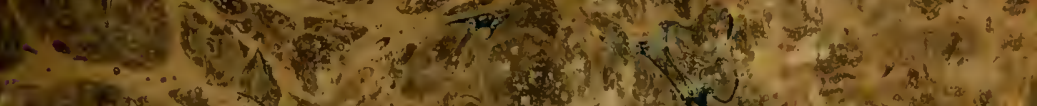

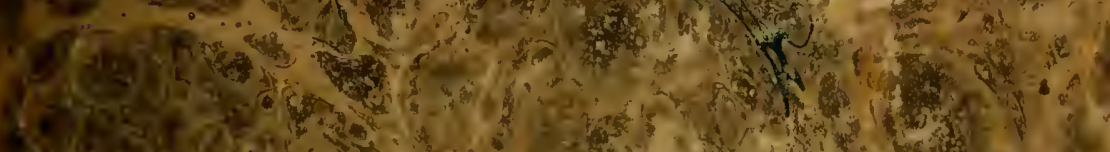

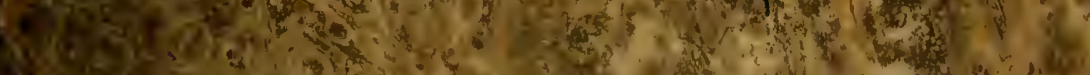
17.

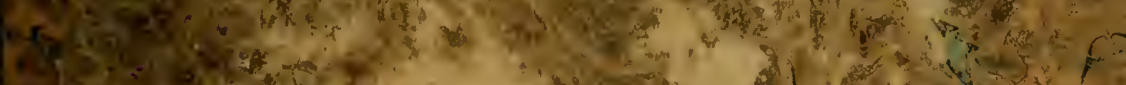

(2)

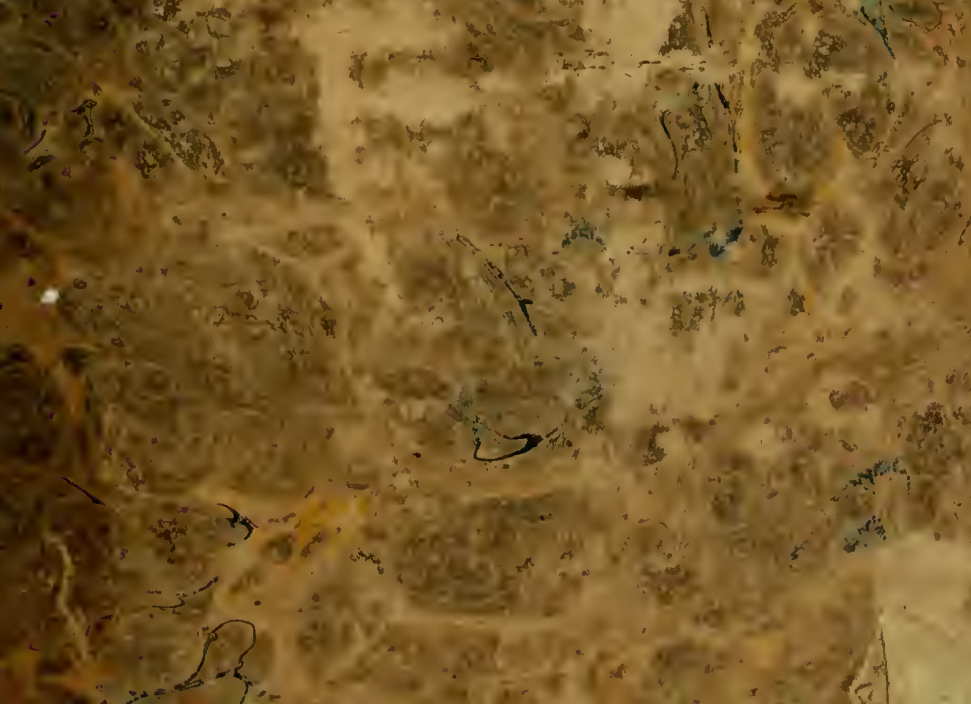


DUPLICATA DE LA BIBLIOTHÊQUE

DU CONSERVATOIRE BOTANIQUE DE GENEVE

VENDU FNN 1022 



\section{MONOGRAPHIE}

\section{DU GENRE ROSIER.}




\section{On trouve cet ouvrage aux adresses suivantes:}

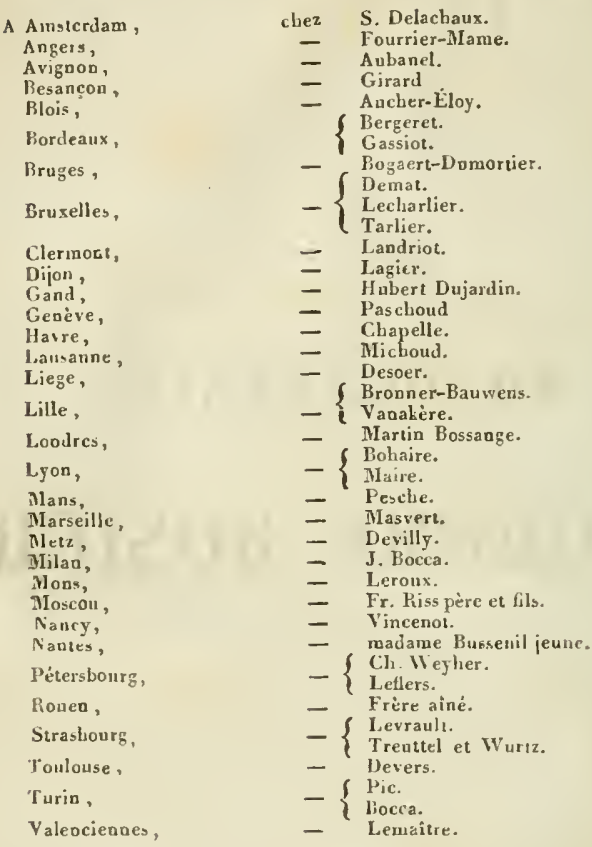

Et cucz les princıpans graimers et marchands tleuristes de Paris.

A Pollwiller, pres Mulhausen, département du Haut-Rhin, cliez MM. Baumann freres, botanistes et pépiniéristes. Cette maison, où l'on peut se procurer tout ce qu'il y a de rare et de nouveau en fait de végétaux, est en relatiou avec les principaux établissentens de culture de toutes les contrées.

A Annonay, département de l'Ardèclıe, clıez MIM. Jacquemę Bonnefon père et fils, malchands grainiers et pépıniéristes, dont la maison mérite a tous égards la confiance dont elle joutt.

A Tarascon (Bouches-du-Rhône), cliez M. Audibert, botaniste et pépiniériste, dont l'ẻtablissement est reconımandable par l’ètendue de ses pépinières et de ses relations.

A Ville-d'Avray, par Serre's, pres Paris, chez M. Codefioy, pépiniériste. Il a des catalogues imprinués.

I'ARIS. - INIPLHERIE DE FAIN, RUE RACINE, N०.4, PLACE DE L'UDÉYY. 


\section{MONOGRAPHIE}

\section{DU GENRE ROSIER,}

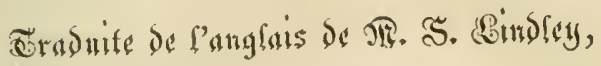

AVEC DES NOTES DE M. L. JOFFRIN, ET DES CHANGEMENS IMPORTANS,

SUIVIE

D'UN APPENDICE SUR LES ROSES

CULTIVEes DANS LES JARDINS DE PARIS

ET ENVIRONS.

\section{PAR M. DE PRONVILLE,}

MEMBRE DE LA SOCIÉTÉ n'agriculture DE versailles et de Plusieurs autres sociétés.

PHIX: 3 fr. 50 c.

Lien AR'

PH:W roth

i3i:raAich

PARIS,

AUDOT, LIBRAIRE-EDITEUR

DE L'HERDIF DE L'AMATEUR, DU BON JARDINIER, elc,

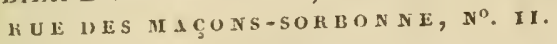

I 324 . 


$$
\begin{aligned}
& .156 \\
& 25 \\
& 1824
\end{aligned}
$$




\section{AVANT-PROPOS.}

La traduction de la Monographie des Roses de M. Lindley a été commencée vers la fin de $10{ }^{22}$. De nouvelles observations faites en 1325 mont engagé à y faire quelques changemens, et c'est ce qui en a retardé l'impression. Enfin cet ouvrage, qui vient d'ètre accueilli par la Société d'agriculture et des arts du département de Seine-et-Oise, va être livré au jugement des botanistes et des amateurs qui ne connaissent pas celui de M. Lindley. Je ne crois pas n'ètre écarté de l'exacte inpartialité dont je me fais toujours un devoir. Je me suis servi des conseils de M. Bose, dont les connaissances en histoire naturelle n'ont pas besoin d'éloges, et des lumières de M. Joffrin, qui a séjourné long-temps en Angleterre et qui a étudié avec fruit les herbiers de S. Joseph Banks, du chevalier Lambert, et le jardin botanique de Curtis.

Les établissemens royaux du Jardin des Plantes et du Luxembourg, la pépinière de M. Noisette et celles 'de MM. Vibert et Godefroy, m'ont été fort utiles tant pour les espèces botaniques que pour la comparaison entre elles des variétés it tleurs doubles. J'ai eu soin de marquer avec des guillemets les passages et les observations qui m'appartiennent.

Mais, dira-t-on peut-ĉtre , dans quelques années le domaine des roses peut s'enrichir de plusieurs espèces nouvelles? Quel est l'ouviage complet sou's 
ce rapport? il n'en existe pas. Nous ne cultivons pas même toutes les espèces qui sont mentionnées dans ce recueil. Mon but a été de faire connaître dans tous ses détails un mode de classification fondé sur des caractères assez saillans pour les avoir choisis parmi beaucoup d'autres; je les ai vérifiés sur la plus grande partie du genre. Je me contente de la satisfaction que j'en ai éprouvée, tout le mérite de l'exécution devant rester au botaniste anglais, lequel, entouré d'aussi nombreux matériaux, a si bien su les mettre en oeuvre.

Le Synopsis Rosarum qui précède la description des espèces, a déjà paru en I 822 sous le titre de Sommaire, et, pour ne rien laisser à désirer sur l'histoire du rosier, j'ai cru devoir rapporter dans l'introduction ce qui concerne les auteurs; cette partie est extraite de ma Nomenclature. 


\section{TABLE ALPIIABETIQUE}

\section{DES ABREVIATIONS.}

AFzel. Tent. prim.

Аїт. Kew.

\їт, Kew, ed. alt.

Aıl. Ped.

Andr. Ros.

Bast. Maine-et-Loire.

Bast. Suppl.

BuUH. Hist.

BadH. Pin.

Belland. Act. Taur.

BesL. Eyst. vern.

Buв. Taur.-Canc.

Bosc. Dict. d'Ag.

Вrot. Lus.

Brown. in Salt's.

Bull. Par.

Cuus. Hist.

Cuus. Cur. post. Crantz. Austr.
$\Lambda$.

Afzelius. De Vegetalibus, etc. Tentamen primum; Upsal.

IV. Aïton. Hortus kewensis, editio prima; London, 1789,3 vol. in-8.

Idem, editio secunda; London, I8ro-13, 5 vol. in-8.

C. Allioni. Flora pedemontana; Taurini , 785 , 3 vol. in-folio.

Monogiapliy of the genus Rosa, by H. C. Andrews; Loodon, i 787 et suiv.

l..

T. Bastaud. Essai sur la Flore du département de Maine-et-Loire; Angers, 1 \$og, 1 vol. in-12.

Ldem. Supplément à la FI. de Maine-et-Loirc, 1 vol, in-12.

S. Bauhio. Historia plantarum universalis; Ebroduni, 165o-51, 3 vol. in-folio.

G. Bauhin. Pinax Theatri botanici; Basilea, ed. secunda, $16 \mathrm{jl}$, in- $\{$.

L. Bellandi. Mćmoires de l'académie des Sciences de Turin.

N. Besler. Hortu's eystettensis; Nuremberg, i612, $x$ vol, in-folio.

Marschal de Buberstein. Flora Taurico-Caucasica; Cherkovix, 18o8, 1 vol. in-8.

L. Bosc. $\Lambda$ rt. Rosier du nouveau Dictionnaire d'agriculture; Paris, 1809 , $13 \mathrm{vol}$. in. 8 , seconde édition, 1821,16 vol. in-S.

Drotero. Phytologia lusitanica selecta; Olisponi, 1801 , Fasc. in-S.

R. Brown. Article du voyage d'Abyssinie par N. Salt.

Dulliard. Plantes figurées des environs de $\mathrm{Pa}$ i's

C.

Clusias ou de Lécluse. Rariorum plantarum Historia ; Antwerpia, $161 \mathrm{I}$, in-folio.

Idem. Curre posteriores, opus post lumum, 1611 . II. S. N. Craut\%. Stirpium austriacorum faseieuli ; Vienne, $1,62-69$. 
Cupan. Pamph. ed. Cort. Mag.

DC. Decand. Fl. fr.

Desf. Atl.

Desf. Cat.

Desv. Journ.

Dill. Eltlı.

Dop. Hist.

Dov. Pempt.

Doos. Cant. ed.

Dom. Cour. Bat. c.

Duroi. Harbk.

EHr. Beitr.

Fl. dan.

Gat. Montanb.

Ger. En.

Ciss. Fad. $-\Lambda$ ls.
Cupani, auteur de l'Hortus catholicus, 1696 . W. Curtis. The hotanical Magazine; London!, $1787-9^{8}, 12$ vol. in -8 .

D.

A. P. Decandolle. Artiele Rosier dans la troisième édition de la Flore francaise, par messienrs de la Mlarck et Decandolle; Paris, 1815 , $6 \mathrm{vol}$. in-8.

R. L. Desfontaines. Flora atlantica; Paris, I $79^{8-99,2}$ vol. in 4 .

Idem. Tableau de l'école de Botanique du Mus. d'Histoire naturelle de Paris; sec. éd., r8o4.

N. A. Desvaux. Observations critiques sur les espéces de rosiers propres au sol de la France, dans le Journal de Eotaniqque; Paris, $18_{1} 3$.

S. S. Dillenius. Hortus elthamensis; Londini, $173_{2}, 2$ rol. in folio.

fiemb. Dodoneus. Historia plantarum tuguriorum; Antwerpiæ, 1569 , in-8.

Ejusdem stirpium historix Pemptades 6; Antwerpia, in-fol.

S. Doon. Hortus cantabrigensis, editioseptima ; Londini; $1 \mathrm{~S}_{12}$, 1 vol. in-8.

Dumont de Courset. Le Botaniste cultivateur, premicre édition; Paris, $1 \mathrm{So}_{2}-5,5 \mathrm{vol}$. in- 8 ; seconde édition, 6 rol. in-8, is I I ; Supplément, 1814 .

S. P. Duroi. Harbkesche Wilde Baumzucht; Brauschweig, $1771-72,2 \mathrm{vol}$. in -8 .

$$
\text { E. }
$$

F. Ehrliart. Beitrage zùr naturkunde; Hanover et Osnabruck, $1787-9 \mathbf{1}, 7$ vol. in-8.

F.

Oeder. Flora danica; Hafnire, $176 r-18,6$, 8 vol. in-folio.

G.

Gattcau. Description des plantes qui croissent aux environs de Moatauban; 1789 , I vol. in-S.

Gerarde. The Hcrball, or génćral History of ['lants inlarged by Johnson; London, 1633 , in-fol.

C.C. Gmelin. Illora badensis-alsatica; Carlsrulie, ${ }_{1}$ So $5-8,3$ vol. in-8. 
Gooan. Monsp.

Goosw. Illust.

HaLL. Helv.

lierm. Diss.

Ilofrm. Deutch. FI.

Huds. Angl.

Hlumb. et Bonpr.

$\mathbf{J}_{\Delta \mathrm{cc} \text {. Obs. }}$

J

JAcQ. Austr.

JAcQ. Schœenb.

Кгоск. Siles.

L.APeyr. Pyi.

LAWR. Ros.

LeErs. Herb.

LeJ. Fl. de Spa.

Lexs. llal.

LightF. Scot.

Lival. Mon.

Liкx. Spec.
A. Gouan. Hortus regius monspeliensis; Lugduni, I 762 , in-8.

Gouan. Illustrationes et observationes botanica; Tiguri, $177^{3}, 1$ vol. in-fol.

H.

A. de Haller. Ilistoria Stirpium indigenarum Helvetia ; Berna, 1568,3 vol. in-folio.

S. Hermann. Dissertatio inauguralis botanicomedica de Rosâ; Argentorati, 1762 , in-4.

lloffmann. Deutchland's Flora; Erlangen, 1791 , 3 vol. in -12 ,

IV. Hludson. Flora anglica ; London, ${ }_{1-62} 6_{2}$, 1 vol. in-8.; seconde édition, 1578 , 2 vol.in-8. Nouveaux genres et espèces de Plantes recueillies en Amérique par MIII. de llumboldt et Bompland.

J.

De Jacquin. Observationes botanicæ; Viennæ, 176 - 71,4 fascic. in -4 .

Ejusdem. Fragmenta botanic $x, 1764$; part. 4, in-4.

Ejusdem. Floræ austriacæ icones; Viennæ, $177^{3} \rightarrow-8,5$ vol. in-folio.

Ideme. Deseription des plantes di jardin impérial de Schoenbrun; Viennie, 1797,2 parties.

$\mathrm{K}$.

$\Lambda$. Krocker. Flora silesiaca renovata, etc.; Uratislaviæ, $r S_{7} \rightarrow 9^{\circ}$, in- 8 .

L.

Picat de la Peyrouse. Flore des Pyrénées, avec fig. : Toulouse, 1794 .

Idem. Ilistoire abrégée des Plantes des Pyrénécs; Toulouse, 1813 , 1 vol. in- 8 .

Collection des Roses gravées et coloriées, par miss Lawrence ; London, $179^{6-99}$, in-folio.

S. D. Leers. Flera herbernensis ; Colonix, 1789 , 1 vol. in-8.

Lejeune. Flore des environs de Spa ; Liége, $1511-13,2$ vol. in 8 .

F. W. Leyser.Flora halensis; Halæ, 1783, 1 vol. in-8.

Lightfoot. Flora scotica; Londini, 1777, 2 vol. in-8.

S. Lindley. The botanical History of Roses; London, $1 \$ 20$, I rol. in-8.

Caroli à Linné. Species Plantarum, ed. prima ; llolinix, 1753,2 vol. in-s. 
Linn. Spec.

Lis. H. Cliff.

Lois. In Desv, journ.

Lour, Coch.

$\mathrm{M}_{\Lambda \mathrm{R} \text { SH. Arb. }}$.

MER. Par.

Micu. Bor. Am.

M!LL. Dict.

Moessch. Meth.

Ne1L. Journ. phil.

Ourv. Voy.

PaLl. In nov, Act. Pctrop.

Pall. Iiuss.

Pers. Syn.

Pet. Gaz.

Pluck. Amalth.

Poir. Enc.

Prosv. Nom.

Proxi. Somm.

Pursa. Am. sepl.
Ejusdem. Editio secunda ; $1762-63$.

Ejusdem. Hortus Cliffortianus.

Loiseleur des Longschamps, auteur de la Flora gallica; Paris, 1807,2 vol. in-12, avec supplément, 18เo. Cité par M. Desvaux, dans le Journal de Botanique.

J. de Loureiro. Flora cochinchinensis ; Ulyssiponi (Lisbonne), $159^{\circ}, 2$ vol. in-4.; secunda. ed., curante Willdenow, Berlin, 2 vol. in-8.

\section{M.}

Marslıall. Arbustum americanum; Philadelphie, $1-58$, in-8.

F. V. Mérat. Nouvelle Flore des environs de Paris. $18_{12}$, 1 v. in-8.; $2^{\mathrm{e}}$. éd. Paris, $1 \$_{21}, 2$ v. in-18.

A. Michaux. Flora borealis americana; Paris, 1803,2 vol, in-8.

Dictionnaire des Jardiniers, par Ph. Niller, Trad. de M. de Chazelles. Paris, 1785,8 vol. in-4., avec supplément, 1789 , 2 vol. in -4 .

C. Moensch. Methodus Plantarum horti et agri marburgensis; Marburg, 1794, 1 vol. in-8.

N.

M. Neil. Article dans le Journal philosophique d'Édimbourg.

0.

Voyage en Perse par M. Olivier, de l'Institut.

P.

P. S. Pallas. Nova Acta Academix scientiarum imperialis petropolitanie.

ldem. Voyage dans l'empire de Russie, 1771 $\rightarrow 6$. Traduit de l'Allemand.

C. H. Persoon. Synopsis Plantarum; Parisiis, 1805-7, 2 vol. pet. in-12.

V. Petiver.Auteur de l'llistoria naturalis, etc. Londini, 1664,3 vol.in-folio.

L. Plucknet. Analtheum bolạuicum; Londini. Encyclopédie méthodique, partie des la botanique, article hosier, par W. Poiret; Paris; 1804 , vol. in- 4 , et supplément par le même.

Nomenclature raisonnce du genre vosier, par M. de Pronville ; Paris, 1818 , in-8.

Sommaire de la Monographie du Rosier par Je même; Paris, ${ }_{1822}$, in 8.

I: P'ursh. llora borealis-americana ; London, 1804, 2 vol, in-s. 
Qór. Fl. hisp.

Ra11. Hist.

RАб. Enum.

+ Ren. Rios.

X Roess. Ros.

Rотн. Germ.

Rox в. Fl, ind. in. Retz. Scand.

Rieign. Act. Laus.

Sal. Ilist. Allert.

Sav. l'is.

Schleich. Cat.

Schranck. Monæ.

Scop. Carn.

Suxer. Helv.

Spreng. Plant. non cogn.

Sмเтฺน. Brit.

Sиiтн.

Tual. Sylv here.

Thor. Prods.

Tounx. lnst.
Q.

Quer. Flora llispanica.

R.

Benj. Kajus. Ilistoria Plantarum ; London , 1686 -I 704,3 vol. in-fol.

Ant. Ran. Enumeratio rosarum circa Virceburgum, etc.; Nuremberg, 1816, I vol.in-8.

P. S. Redouté. Les Roses avec le texte par M. C. A. Thory; Paris, 1817,2 vol. in-folio.

Roessig. Les Roses avec traduction du texte allemand. Io fasc. in-4. Leipsick, $1800-17$.

A. IV. Roth. Tentamen Florx germanicx; Lipsix, $1788-1801,3$ vol. in-8.

Roxburgh. Flora indica, manuscrit ; Londres.

A. S. Retzius. Floræ scandinavix Prodromus. llolmix, 1779, in-8. - Seconde éd., Lipsia , $179^{5}$.

L. heigner. Description de quelques espèces dc Rosiers dans les mémoires de la Société des sciences physiques de Lausanne; i 784 .

S.

R. A. Salisbury. Prodromus Stirpium in horto ad Chapel Allerton viventium; Londini, $179^{6}$, in-8.

G. Savi. Flora pisana; Pisis, 1598, 2 vol. in-S. Schleicher. Catalogue des Plantes de la Suisse.

F. P. Schranck. Auteur de la Flore de Bavicre; Munich, 1789 .

S. Scopoli. Flora carniolica ; Vienna, 1772 , 2 vol. in-8.

S. R. Suter. Flora helvetica, etc ; Turin, 1802 , 2 vol. in-12.

Sprengel, aulcur d'une llistoire de la Botanique ancienne et moderue en allemand.

S. J. Smith. Flora britannica; Londini, 1800 $-4,3$ vol. in- 8 .

Idem. Edition de l'Encyclopédie de Rees; Londres , $179^{\circ}$ et années suivantes.

\section{T.}

Thalius. Icones Sylva liercinix. Francofurti, I 585, in-4.

Prodrome de la Monographie du genre fiosicr, par M. Thory; Paris, 1820 , 1 vol. in-12.

S. Piton de Tournefort. Institutiones rei herba- 


\section{viij}

Tourn. Coroll.

riæ; Parisiis, $1217-19$, curante $A$. de Jussieu, 3 vol. in -4 .

Ejusdem. Corollarium institutionum rei herbarix; Parisiis, 1 ; 03 , in -4 .

Thurl. . Par.

Vent. Cels.

V1LL. Delph.

S. L. Thuillier. Nouvelle flore des environs de Paris; 1599,1 vol. in-8.

$$
\text { V. }
$$

E. L. Ventenat. Description des Plantes nouvelles $\mathrm{du}$ jardin de M. Cels; Paris, 1800 , in-folio.

Villars. Histnire des plantes du Dauphiné; Grenoble, i $76-88,3$ vol. in-8.

\section{W.}

IV $A$ LDEST. et KIT. W18. Werth.

WILLd. S. pl.

IVILLD. Enum.

Waldestein et Kitaibel. Flora hungarica.

Wibel. Primitiarum Flore Wertheimiensis Prodromus. Senæ, I 797 , in-8.

Caroli à LinnéSpecies Plantarum, curante C.L. Willdenow; Berolini, 1797-1810, 5 vol. in-8.

Ejusdem. Enumeratio Plantarum horti regi botanici beroliniensis; $1809-13,2 \mathrm{v}$. in-8. 


\section{MONOGRAPHIE}

DU GENRE ROSIER

FONDE SUR DE NOUVELLES OBSERVATIONS DE'S BOTANISTES

La Rose est la reine des fleurs. L'Orient, berceau des premier's hommes, est aussi sa patrie, et.les coteaux fleuris sur lesquels s'appuie la chaine sourcilleuse du Cancase se sont pares les premiers de ce charmant arbuste, et donnaient en même temps leur nom à la plus belle race humaine. La rose a été célébrie dans tous les siècles; elle a offert aux naturalistes le sujet de compositions qui joignaient au mérite d'une discussion approfundie celui de détails intéressans pour les gens dı monde. Chacun d'eux a pu se dire comme le Corrége : anch' io son pittore. Quelquelois mêne des botanistes, animés par les grâces du sujet, ont revêtu leurs descriptions des couleurs les plus brillantes (1). La littérature et la poésie surtout se sont empressées dans tous les temps de chanter ce végétal, le plus bel ormement de nos parterres et de nos bosquets; elles lui doivent leurs plus charmantes productions. Anacrion et ses traducteurs, Mu. Henri Étienne, Paolo Rossi, de St.-Victor, ainsi que Thompson, l'abbé Delille, Ducis, Bernard, de lemze, etc., ont épuisé toutes les ressources de la poúsie descriptive, et semblent n'en laisser atlcune à leurs successeurs pour compléter l'histoire et le panégyrique de la rose (2).

1)epuis Hippocrate, qui nous a parlé des vertus de ce végétal, bien des volumes ont été écrits sur ses propriétés médicales, et l'un de ses plus ardens défenseurs n'a pas hésité d'assurer que la pharmacopée devait se réduire au rosier. S'il est

(i) Voyez l'article de M. Poiret, dans le supplément de l'Encyclopédie methodique, tome IV, 2 e, partie.

(2) Ainsi que les organistes ambulans s'cmparent des airs favoris de notre Opéra-Comique, de même les compositeurs d'almanaclis ont ronlu célébrer la rose; mais parmi ces productions, Ic petit livre de M. Charles Malo mérilc' d'être distingué. 
judicicux d'écarter de notre récit tons les contes riöicules quî out été débités sur la rose, et dont un médecin de Nancy s'est empressé de grossir ses ourrages, il est de notre devoir de rappeler en peu de mots ce que les anciens avaient fait pour la ruse.

Comme emblème de la jeuncsse, la rose était dédiée à l'Aurore; elle l'était à Vénus sous celui de l'amour et de la beauté, ct des grâces légères à Cupidun. Elle fut donnée par ce dernier comme une amorce à Harpocrate, dieu du silence, d'où est renu peut-être l'usage rapporté par Rusenherg, et qui arait licu chez les peuples d" Nord, de suspendre une rose au plafond, au-dessus du haut bout de la table, lorsqu'ils voulaient que ce qui se disait entre enx dans leurs festins demeurât secret. C'est sans doute aussi l'origine de l'ancien dictun : sous ta rose. La mythologie, qui embellit ses fables, et qui nons dunne une si haute idce de l'imagination des Grecs, a supposé qu'originairement les roses étaient blanches, mais qu'elles furent colorées par le sang de Vénus, blessee au pied par leur's aiguillons en voulant soustraire Adonis aux fureurs de Mars. "Toutefois, Théophraste thion pensent que ce fut le sang d'Adonis qui en altéra la blancheur: D'autres rapportent que l'Amour se laissa tomber en dansant devant les dieux, et qu'il renversa un vase de nectar, lequel, en tombant sur la terre, colora les roses.

“ L'Olympe, en le voyant, sourit,

"Et sur la plante répandit

"Du nectar la douce rosće.

( SAIN'T-YICTOR, trad. d'ANACPEON.)

Les Turcs ont une superstition d'un autre genre, ct, au rapport de Buslueck, ils atiribuent l'origine de la rose à la sueur de leur prophite Mahomet.

Une rose d'or étuit regardée comme un piésent si honorable que les rois seuls pouvaient la recevoir. Elles étaient consacries par les papes le jour du vendıedi saint pour être offirles aux souverains dont ils voulaient ménager la hante protection. La flem était un emblème de la fragilité du corps, comme le métal dont elle était composée celui de l'immortalité de l'âme. Boécie dit que Guillaume, roi d'Écosse, en reçut une du pape Alcxandre III; l'histoire cite encore de pareils dons. Le cachet du fameux Luther, qui représentait une rose, avait la même signification. Les empereurs romains employaicnt des roses pour hororer le talent et la valeur de 
Leurs généliux, auxquels ils permettaient d’ajouter cette fenr aux ornemens de leurs boucliers : coutume qui a subsisté long-temps après la destruction de l'empire, et dont il reste encore des vestiges dans les armoiries de quelques anciennes familles de l'Europe.

"Les ducs et pairs, dit Sauval, soit qu'ils fussent princes ou mêne fils de France, les rois et reines de Nalarre, étaient jadis obligés de donner des roses an Parlement en arril, mai et juin. On ignore la cause d'une semblable coutune, et l'on n'est pas nour plus lort instruit sur la manicre dont elle s'observait. Nous sommes senlement rertains que le pair qui étilit appelé à faire cetle cérémonie fuisait joucher de roses, de fleurs et d'herbes odoriférantes, toutes les chambres slu parlement, et avant l'audience réunissait dans un déjeuner splendide les présilens, les conseillers, et unême les grelfiers et huissiers de la cour. Il allait ensuite dans chaque chambre, faisant porter derant lui un grand bassin d'argent, lequel contenait antant de bouquets de r'oses, d'oeillets, et d'autres fleurs de soie ou naurelles, qu'il y avait d'otliciers, avec un pateil nombre de conronnes composées des mêmes fleurs rehaussées de ses armes. On lui donnait ensuite audience dans la graud'chambre, puis il assistait it la messe avec le parlement entier. Tant que durait la cérémonie, l'audience exceptíe, il y avait un concert de hautbois qui allait ensuite donuer des sérénades aux présidens avant leur dîner. Il faut abserver de plus, $1^{\circ}$. que celui qui écrivait sous le greffier avait son droit de roses; $2^{\circ}$. que le pulement avait son faiseur de roses appelé le rosier de la cour; $5^{\circ}$. que les pairs devaient acheter de lni celles dont se composaient leurs présens. La présentition les roses se faisait généralement pour tous ceux qui avaient des pairies daus le ressort du parlement de Paris.

"Sous le règne de Francois $I^{\text {er }}$. il y eut, dit Ilénault, dispute entre le duc de Montpensier et le duc de Nevers sur la bailtée des roses au parlement. Le parlement ordonna que le duc de Montpensier les baillerait le premier, à cause de sa qualité de prince du sang, quoique le duc de Nevers fât plus ancien pair que lui. Parmi les princes du sang qui se soumirent a cette cérémonie, on compte encore les ducs de Vendôme, de Beaumont, d'Angoulême et beaucoup d'autres. On trouve même qu'Antoine de Bourbon, roi de Navarre, s'y assujettit en qualité de duc de Vendôme. Heuri IV, n'étant encore que 
rui de Navare justifia an procureur général que ni lui ni ses prédécesseurs n'avaient jamais manqué de satistaire à cette redevance. Elle a eessé entièrement dans le XVIIe. siecle sans qu'on en puisse fixer précisément l'époque. Il y a quelque ipfarence que ce fut sous le ministère du cardinal de Richelicus. »

( Tabl. hist. de Paris, 1. 1., 154.)

Comme ol,jet de culture elles ont toujours été tris-recherchérs, et, dans le dessein d'en ifugmenter la leautí, on u'a négligé dans la pratique aucun moyen pour en oblenir des fleurs doubles. bans les temps inodernes, on al vu paraîtue une mulitude d'individus répandus dans tous les jardins, dont la beauté ne peut être comparée qu'i l'extrême difficulié de remonter à leur souche originaire. Ce sernit une erreur de croire que les roses doubles sont d'une origine móderne, puisqu'il en est fait particulièrement mention par Hérodote, Alhéuće et Théophraste, et notamment par Pline, qui en compta plusieurs espèces, dont le $R$. centifolia. Il est singulier qu'il n'ait fait aucune mention de la rose de Pastum ni d'aucune de celles qui croissent dans celle partie du royaume de Naples. Une pareille omission rend impossible de décider sur le bifera rosaria Pcesti de Virgile. Le sempervi. rens est le seul rosier que M. Woods ait trouvé dans les euvirons de Prstum.

Le now de rose est dérivé, selon Théï, du celtique, rhood ou rhudd, qui signifie rouge, d'oùil pense que sont dérivés les synonymes, en langue armorique rhos, rhodon en grec, et rosha en slavon.

les botanistes moderues se sont atiachés à décrire les espices conuues de leur temps, les uns par des icones ou figures gravées, les autres par de bomes énumérations on des descriptions détaillées. On trouve dix figures de rosiers représentées daus les I cones stirpium (1) de Lobel. Dix-neuf espieces et un plus grand nombre de variétés soni indiquées dans le Pincix (2) die C. Bablin. Hurray, continuateur de Linne, ('n décrit vingt et une espèces dans son Systema v'e. getabiizum (3); mais d'autres botanistes plus récens, tels que Willdenow et Persoon, en ont augmenté le nombre en profitant des nouvelles dícouvertes el eu faiscint eux-mêmes

(1) Plantarum sen stippium Icones. Tom. r. 2. Antwerpix. 1581-9r.

(2) Car. Bauhini, Pinax theatri botanici. Basilce, 1620.

(3) C. Linsé, System. vegetabilium, curante So. And. Murray, rol. in- $8^{\circ}$. Lioltiuge. $178 \%$. 
de nombrenses rechordes. Le Species phantarum (1) du premier contient trente-neul espéces de rosiers at ic Synopsis plantarum de Person quarante-six. Parmi les botinistes cultivateurs, il faut distinguer Miller (2), dout le dietionnaire a été traduit et augmenté d'un supplément par M. de Chazelles, amateur. Cette edition, qui date dejai de 1985 , renferme trente et une espreces. Ce que Miller dit des varictés donbles cultivies dans le jardin de. Chelscia et dans cenx des riches particuliers d'Angletere, litissait heaneoup i disirer; mais $\mathbf{M}$. le professeur Martync, de l'université de Cambridge, qui a entierement refondu cet ouvrage, en a fait disparaîte une foule de répétitions inutiles, et il a désigné d'uac manière plus précise les nombreuses variétés que l'on cultive dans sa patrie; il les a placés dans un ordre plus naturcl en se conformant toutefois a celui de Willdenow. La plupart de ces espèces et variétés avaient été gravées à Londres el coloriées d'apres nature par mademoiselle Lawrence. Cut ouvrage remarquable est pourtant inféricur à la belle collection des passiflora que mademoiselle Lilwrence avait dijii publiée.

Mais nous arons aussi nos Miller. M. Dumnont de Cinrset, qui cultive pris de Boulogne, avecautant de succis yute de discernement, une des plus belles collections de vingétaux que nous ayons en France, a donnć dans son Bolaniste cultivateur (3), une description de trente-sept espèces de rosiers. Ses observations sont justes pour la plupart, et confirmées par sa propre expérience. L'Fncyulopédie méthodique, cet immense magasin des connaissances humaines, levait renfermer la botanique. Cette partie a été supérieurement tritéc par M. de la Marck, dont le nom est cher aux naturalistes et que des circonstinces du temps forcirent it abandonner l'ítude des plantes. Ce dictionnairc, continué arec les mêmes soins par H. Poiret, lait monter le nombre des espéces à soixante-cinq, sans compler ni éaumírer donze autres moins connues. Nous pensons que, pour éviter une partic des nombreuses difficultés que présente lit nomencli-

(r) C. Linné, Species plantarum, cur. C. L. Willdenow. Berolini. $1797^{-1810}$

(2) Diclionnaire des jardiniers, de P. Miller; trad. de l'Anglais par une société de gens de lettres. 10 vol. in-4\%. Paris. 1785.

(3) Le Dofaniste cullivatcur; par M. Dumont le Courset, 2 . d 6 vol. in-So, Paris, $18 \mathrm{I}$. 
ture, il serait prudent it un botaniste de n'admettre comme espèces que celles qui sont non-seulement décrites d'une manière précise mais encore dont on peut vérifier les caractères distinctifs sur des échantillons bien conservés, si on ne le peut toutefois sur les plantes vivantes, ce qui vaudrait infinim.nt inieux. Il faudrait même, prour appuyer son opinion, donner des figures exactes, comme l'a fait M. Lindley dans sa monographie, dont nous parlerons avec détail. Le nombre des expèces ne serait pas beauconp diminué, mais la classification deviendrait plus facile.

Ilais un ourrage qui a complété la nomenclature et les connaisances sur l'édocation du rosier, est le dictionnaire d'Arriculture dans lequel $\mathbf{M}$. Bo:c, auteur de cet intéressant article, a dissipé en grande partie l'obscurité que la culture a répandue sur les especes et variétés de ce bean genre. Il en decrit quarante-deux, parmi lesquelles on en trouve qui n'araicnt pas été déterminćes avaut lui. Ies rapports et les diflërences spécifiques sont discutés arec celte sagacité qui n'appartient qu'au prolesscur exercé, et l'examen approfondi que l'auteur a pu faire, surtout depuis qu'il lirige les pépinieres du gouicrnement, doit domer plus de poils à ses décisions.

Dire que M. Redouté a commencé et qu'il poursuit arec persévérance une collection de figures iles principales especes et variétés de rosiers, c'est annonicer un ourrage digne de l'auteur des Liliacées, des Jardins de Cels et de Malınaison; 0دvrage supérieur, au moins quant aux figures. à cetix de l'arkinson, de Roessig et de Mlle. Lawrence. On doit louer le discernement et la concision de l'auteur du texte, M. Thory, qui a rassemblé en peu de pages toul ce que les amateurs pouvaient apprendre d'intéressant sur chacune des especces que M. Redouté a dessinées. L'ouvrage ne devait se composer dans le principe que de cent vingt figures, mais les nombreux matériaux des aulenrs les ont engagés à étendre beaucoup cette belle collection.

Les connaissances que cet ouvrage a procurées à M. Thory sur les rosicrs, partie dı règne végétal qu'il arait dija étudiée, l'out ungagé probablconent á donner au public une monographie à laquelle il travaille encore, mais qu'il a fait précider d'un prodrome (1) ou idée du système qu'il se propose de

(1) Prodrome de la monographie des espices et rariétis cornues du 
suivre. Ce systime ne s'aceorde point arec celni qui tait l'objet de cette traduction.

Ce système se compose de 5 paragraphes et de 2.5 groupes. Les paragraphes sont les réunions artificiclles qui ue se lient poiat ensemble, puisque le premier a rapport aux diflerentes situations des liges: le second est itabli d'après les diver'es modifications des lolioles; le troisicuse, d'anrés les inodifications des tubes turinines ou accompionés de hractees; Ic quatrième est fondé sur les considérations des étamines; et le cinquième d'apris celle des styles. Ces grandes divisions, dont plusieurs n'embrassent point un grand nombre d'espèces, sont dejji un inconvénient pour l'orlte général.

Sur 25 grroupes, 11 a 12 ne sont composés que d'une seule espèce, ce qui ferait douter si l'auteur a voulu grouper des espèces ou des variétés. Mais les variétés, se rattachant aux espèces qui les ont produites, ne doirent pas être comptées dans les divisions générales, surtout si l'ouvrage est écrit antant pour les botanistes que pour les cultirateurs. Je crois aussi que les termes de la science doivent être justes dans leur emploi, surtout iorsqu'ils ont déjà une acception générale. Ainsi le not tube employé pour ovaire ou fruit me paraît défectueux; ce mot signifie, d'après les meilleurs lexicographes, tuyau, conduit; il est alfecté particulièrement à ceux de verre ou de métil dout on se sert dans les expériences; et, de tous les fruits connus des rosiors, je ne vois guère que celui du damasecna (4 saisons), anquel i! pourrait convenir. Je n'en fais pas un reproche i H. Thory, car H. Lindley s'en est servi lui-même (tube of the calix), et je me suis vu forcé de l'imiter.

Si l'on a trouví que le terme division calycinale était trop Inng, j’aurais désiré qu'un autre mot que lanière l'eat remplacé ; car il ne peut être juste que pour la série clés cinnamomce, et non généralement. car ces divisions sont trèscourtes dans le gallica, l'arvensis et plusiemrs antres; je me servirai de sépate (sepaliı) avec l'adjectil qui les caractérise.

La R. rapa de lose ne sanrait être placée parmi les turbinate. Son fruit est constamment alrondi à sa base, même dans sa unaturité; il n'a done point la forme d'une toupie a jouter, caractere de ce groupe.

Que l'onme permelte de douter que Iespomponiance puisgenre Rosier; par Cl. Ant. Thory, membre de plusieurs sociétés savantes. Paris, 1820 , vul. in-12. 
sent constituer un groupe : je pense que les plantes qui le composent appartiennent à des espéces diflértutes. Ainsi la rose de St.-François est évidemment un parvifolia, comme le pompon des fleuristes est une variélé du centifolia. La rosa parvifotia de Willdenow peut être consilérée comme espèce. de vouldr is aussi que l'on supprimât les noms de pays comme trop ragues, surtout lorsqu'ils rappellent à l'obserrateur un trop grand nombre d'espéces : je veux parler ici dı septième groupe americana, et du $24^{\circ}$. indice. Quant au $10^{\circ}$ hudsoniance, qui n'est composé que d'une espèce, comme elle est dédiée an célèbre voyageur de ce nom, cela détruit toute ambiguïté. Je tronve en général, et pourme résumer, que la plupart des divisions de ce système sont fondées sur des caractires trop minutieux et trop rariables pour éclaircir les difficultís qui s'accumulent de jour en jour. Mais les réunions sont artificielles, comme l'auteur le dit lui-même; il les a établies dansle but louable sans doute d'aider l'amateur à soltir du labyrinthe formé par les diverses théories; mais je doute que, malgré son esprit d'observation, il atteigne ce but si désiré.

Il n'en est pas de même de l'ouvrage de Bl. Lindley. Cette monographic, quoique présentant, comme tout ce qui sort de l'esprit humain, quelques côtés faibles, est au moins fondée sur des rapports naturels assez tranchans. Elle l'est aussi sur une foule d'observations neuves, et dont la plupart mont paru très-justes. M. Lindley a vait ì sa disposition des matériaux que l'on chercherait vainement en France, les herbiers les plus précieux, lont celui de linné, que possède, ainsi que ses manuscrits, S. James Smith, les vastes collections de Banks, du chevalier Lambert et de il. Sabine, des communications des naturalistes voyageurs sur les espèces asiatiques peu connues, des recherches inultipliées sur l'organisation des p'antes vivantes faites a diflérentes époques : tous ces matériaux bien employés donnent une grande im. portance a l'ouvrage de M. Lindley. Ses observations sur les espéces connues militent en fareur de celles qui ont trait i lles espèces étrangères, et que nous pourrons peut-être vérifier anssi Jorsque la seience recevia plus d'encouragement.

M. Lindley, ainsi que II. Thory, et avant eux M. Poirct, a cru devoir négliger les deux grandes sections linnéennes relatires à la forme des fruits. "On a cru jusqu'ici, dit-il, tirer des fruits des caracteres très-évidens, et il n'y aumit 
rien de micux si cescaractires et nent constans. Ilulhenrusement, ilarrive que peude parties de la plante sout allssi sujettes i varier, non-seulement quant a la surlace, mais anssi quaut a la torme et à la dimension. Cette remarque s'appliqua spicialement aux tomentosa, canina et rubiginosa, où l'un trouve toutes lés diversités de furme, surfiace, ete. Cependant quelques espèces sont beancoup moins polymorphes. Mins soit que nous les commaissions moins, ou qu'elles soient moins sujettes à varier, je u'oserais pas décider sur ce point. res cinnamomea sont un exemple de la plus grande constance ile formes, les canina et villosa. an contraire, présentent les formes les plus variables daus leurs fruits."

Je serai plus harui que M. Lindley, et je ne balancerai pas a admetire au moins la première section les fruits grlobuleux ou obronds. Presque toutes les espècos renfermées dans les cinq premières divisions de Lindley conservent cette forme; elle se retrouve aussi dans quelques espéces des antres divisions; mais dans celles-ci l'on peut remarquer aussi d'autres formes. C'es considérations m'ont porté,par respect pour la mémoire de Linné, ¿̀ conserver a la premiere section le caractice de fruits globuleux ou obronds, et de fruits ovales ou variables a la seconde. Les folioles des rosiers de IIIa première section sont généraleınent plus petites (le $R$. parvifolia excepté) que dans la seconde; ces mêmes folioles sont lisses, souvent luisantes dans cette premiere section, ce qui est aussi moins cominun dans la seconde. Les divisions calycinales (sépales) sont presque toutes simples et prolongíes en alène au delá du bouton et même de la fleur épanouie. Ce sont des caractères assez constans et allssi sors que ceux des aiguillons qui disparaissent des vieux raneaux, et aussi par la culture, puisque l'on a aujourd'hui des $R$. diemascena qui en sont presque dépourvus. M. Decandolle, dans sa Théorie élémentaire, convient que la considération des aiguillons est trop variable pour s'y arrêter sérieusement, quoiqu'elle le soit moins que la présence on l'absence des poils sur une ou plusieurs parties de la plinte. Cependant H. Lindley a cru ces caractères d'une assez grande valeut pour les employer dans les divisions de sa monographic, et j'ai vérifié sui toutes les espéces que je possède nne asse grande constance dins ces calacteres pour les employer aussi.

La sixième division de Lindley présente dan s ses espéces. ainsi que les suivantes, des fruits ovales ovoides, en parlic 
variables. Ce sera ma deuxieme section a fruits ovales ot rariables. Elle renferme ¿i peu près la moitié des espèces. Ces deux sections me paraissent naturelles. Je ne ferai pas comme le bon M. Buchoz, qui ne reconnaissait que deux espices de rosiers, l'une sauvige, et l'autre cultivie; mais le's onze tribus dont se compose le genre auraient pu être considérées par ce médecin comme des espèces, et leurs caracteres généraux se rapporter à ceux des especes proprement dites.

Le titre de cet ouvrage annonce qu'il est entrepris d'après celıi d'un naturaliste fort exercé, mais dont les travaux ne sont point connus en France. J'ai adopté sa méthode à quelques modifications près, et j’ai fondu, en quelque sortc, ses observations avec colles que quinze ans de culture d'une collection considérable m'ont offerts. Quoique je ne partage pas entierement ses opinions relativement aux deux sections fondées sur la forme des fruits, il est de mon devoir de faire connaitre les raisons sur lesquelles il appuie son système, afin que les lecteurs puissent fixer leurs idées sur son importance.

Les documens que nous avons sur les roses européennes, et les recherches nombreuses dont elles ont été l'objet, sont devenus si étendus, que l'on peut douter qu'il existe dè viritables limites entre ce qu'on appelle les espèces. C'esi ce que soupconnait fortement Linné quand il a dit : Species timitibus difficillimè circumscribuntur, et forle natura. non eas posucrit.

Mais il n'avait aucun moyen de se satisfaire à cet égard. Gérard et d'autres l'out assuré, quoique la vérité de cette opinion ait été contestée par Haller, et la plupart des botanistes qui sont venus après lui. Un examen partiel, mais satislaisant, peut faire découvrir le chainon qui unit les espèces européennes aux étrangères. Les roses canina et spinosissima peuvent être prises pour exemple el faire voir les points de différence el de resemblance. Commencons par le spinosissima. Ce rosier est réuni au $R$. rubella par la variélé melanocapa du dernier. Sa variété pilosa le lie avec le R. involuta, lequel, dans un étal plus vigoureux. devient le doniana de Woods, chainon intermédiaire entre linvoluta et le Sabini. Celni-ci devient villosa par le $R$. gracilis qui lient de l'un et de l'autre. Par un retour particl a son apparence origindire, c'est-i-diri, en perdant ses poils, mais 
conservant ses glandes, le $R$. involuta devient le myriacantha, autre brinche du spinosissima, en p.ıssint par le rubella, qui devient $R$. alpina par la variéte naine de ce slernier.

Avant l'aller plus loin et d'examiner jusqu'i quel point ces circonstances peurent influer sur la classitication du genre, il n'est pas hors de propos de chercher ce que l'on doit entunilre par espèce. Cuvier nous a dit que e'est l'umion d'individus des. cendus l'un de l'autre, ou de parens communs, ou de ceux qui leur ressemblent comme ils se ressembleut l'un l'autre. (lìgn. anim., 1.19.)

Decandulle défnit l'espece: un assemblage de tous les individus qui se ressemblent plus qu'ils rie ressemblent à d'autres et qui peuvent, par une fécondation réciproque, produire des indivilus fertiles, susceptibles de se reproduire d'euxmêmes par la génération, de manière que l'on puisse supposer, par analogie, que tous sont descendus originairement d'un seul individu. (Théor. élém. ed. 2 , 193.) Maintenant, si ces défnitions non hypothétiques sunt le criterium par lequel une espèce doit être jugée arant que d'être adınise comme telle, il résulte des principes que j'ai énoncés que toute la partie européenne du grenre, doit être coutondue ensemble sous un titre spécifque, mesure dont l'absuritite saute aux yeux, puisqu'en l'adoptant il n'en résulterait qu'une dispute de mots ; car il serait toujours nécessaire de distinguer certaines modifications auxquelles il importerait peu de donuer le uoun d'espèce ou de variété.

Pourquoi distinguons-nous les espèces des genres, si ce n'est pour donner de la précision à nos idées, et tenir un lanrage correct en indiquant certaines modifeations de la structure, mais d'une importance inférieure à celles qui distingnent les genres, et dont les limites supposées sont déterminées par ce qu'on appelle le caractere spécifique.

l'ar espéce, je désire que l'on entende un assemblage d'individus quii différeut sous plusieurs rapports du reste du genre, mais ayant entre eux plus de points de contact qu'avec d'untres, leur union étant d'après cela naturelle. Hais, comIne j'ai essayé de le faire voir, il n'y a point de limites entre les espèces, et il est impossible de donner des définitions rigoureuses. C'est dans la ferme conviction de cette vériti que j'ai contrepris la révision du genre, et sur ces principes que j'ai composé cet ourragre. 
Eu examinant les causes du desordre qui rìgne dans la classification, je me suis assuré qu'une grande difficulté ponvait être résolue en cessant d'insister sur les faibles distirictions a l'appui desquelies nombre le botanistes ont etabli leurs espèces. Il est évident que là où l'on ne peut sáisir qu'un pelit nombre de caractères vagues, une pareille marche, loin de leur donner quelque importance, les divisera jusqu'à ce qu'ils soient confondus. Il ne suffit pas pour constituer une espèce, qu'elle puisse être distinguée de toutes les autres par un eultivateur expérimenté; car, s'il en était ainsi, la plupart des nombreuses variélés de poinmes, de poires et de prunes qu'un jardinier reconnaît facilement, auraient droit, a vec rai. son, à des distinctions spécifiques.

Une seconde cause de confúsion vient le Linné lui-même, lorsqu'il partagea les espèces en deux divisions, distinguées par les ovaires ou fruits (germinibus) ronds ou ovales. Il était dilficile de se fixer sur un caractère plus variable, et cependant il a été adopté, à quelques exceptions près, par la plus grande partie des botanistes.

Quelques exemples prourent que l'on a cssayé de changer cet orlre; mais comme le plan proposé n'était guère meil- leur que celui de Linué, il a obtenu peu de succès, en sorie que dans les nomenclatures complètes du genre qui ont paru depuis et dans l'Encyclopédie des roses, rédigée par Suith, l'ancien mode de livision a prévalu (1).

M. Woods a été le premier qui ait rejelé les préjugés en faveur de cette inéthode, et, dans un excellent mémoire sur les roses de l'Angleterre, il les a déterminées et arrangées d'après un système qui lui est propre. Quelque dificulté qui existe enire nous relativement aux limites des espèces, il ne peut $y$ aroir que pen ou point de différence d'opinion sur les divisions primaires, parce qu'elles sont naturelles. Il a d'abord signalć quelques caracteres importans, parmi lesquels les poils et les aiguillons méritent d'être mentionnés. Je reconnais a vec satisfaction l'arantage que j'ai tiré de ce système, ot, pour montrer l'importance que j'y altache, je l'ai pris pour base du mien. Il est vrai que les alditions et changemens que j’ai cru devoir y fiire ont élé considérahles; mais ils sont tels que M. Wood lni-même les aurait probablement adoptés s’il cot filit entrer daus son plan les échantillons cxotiques. Il

(1) Voyez ce que j’ai ćcril page 9 
a vone cependiut que ses divisions sont faites sans avoir égard aux roses de la Graude. Bretagne, et à cet igard son arrangement est défectueux.

En 18ı, un an après que M. Wood eat lu son mémoire à Ia Société linncénne, le docleur Ambroise Rau publia son Enumération des roses des environs de Wurtzbourg, disposćes d'iprés une nonvelle méthode. Ses remarques sur les espèces snnt exactes et utiles; mais le mode de classement me farait vicieux. Cot ouvrage mérite pourtant de fixer l'altention par le soin que l'auteur a mis en tratant sou sujet. Ce sont les deux seuls essais de classification du genre Rosier, dont il est nécessaire de parler. Dans' l'un et l'autre les espéces sont beaucnup trop multipliées el quelques-uns de leurs caractères sont peu satisfaisans.

Dans la disposition suivante, mon but principal a été de les r'endre naturels. Pour l'effectuer il fallait chercher à connaître toutes les espìces et les soumettre individuellement à une analyse scrupufeuse qui me mettait en état d'assurer jusqu’à quel point les rapports généraux el particuliers inarchaient ensemble.

De ces caractires qui sont tirés des meilleures et plus constantes espèces, j'ai choisi les plus saillans. Ce n'est point à moi à décider si je l'aj fait d'une manière satisfaisante. Je suis pourtant convaincu de la nécessité d'observer les plantes vivantes pour obtenir une lonne classification de rosiers (1). Cependant je soumets au jugement des botanistes une série d'observations sur la perminence respective, ou la disposilion à varier des modifications de chaque organe particulier.

Quoique le port (habitus) des rosiers ue soit pas instantané, il peut être quelquefois employé utilement quand ses différences ont pour cause la croissance des drageons. Lursqu'ils sont courbés, l'on distingue les canina el les rubiginosa des 'villosa dans lespuels ils sont droits. Les rejetons flagellilormes de l'arvensis empêchent de le confundre arec le systila, et leur disposition a lamper sépare le sempervirens du prostrata. Néanmoinz le cinnamomea renferme des plantes dont l'une a les rejetons droits, et les autres

(1) C'est un grave inconvénient sans doufe de ne pouvoir observer que sur le sec; mais, à quelques égards seulement, il est inćvitable. Nous serions moins embarrassés si tous les botanisles s'en étaient tenus là, et n'avaient pas grossi leurs prodromes d'espéces publiécs sans éclsantillons ni ligures gravécs. 
courbés. La même remarque est applicable au tomentosa.

J'ai cru nécessaire d'établir une distinction entre les branches (rami) el les rameaux (ramuli), entendant par les clernicrs toutes les pousses latérales qui sont produites dans la même siison; aiusi le $R$. lutescens est bien distingré du spinosissima par les aiguillous serrés de ses luranclses et la rudesse de ses ranteaux. Dans le $R$. laxa les branches sont dipourvacs d'aignillons. Le lucida est giani d'aiguillons infrastipulaires, le R. rubella est armé jusqu'i ses extrémités. Dans l'espéce la plus voisine, $R$. stricta, les rameaux sont presque nus. I.e R. histrix les a entièrement couverts de petits poils rudes, tondis que les branches en sont totalement dépourries.

Armaest un terme qui exprime la prísence des poils et des aiguillons mêlés sans ordre. Setwe sont de petits aiguilions droits terminés par une glande. Elles se distinguent des glandes rélles par leur rigidité, une plons grande dimension et leur temlance à se convertir en aiguillons. Elles existent, je crois, à quelques périodes dans toutes les espéces sur les jets prorenant des racines où elles sont promplement converties en aiguillons par la perte de leurs glandes. Lin général, elles disparaissent au bout d'un an. Plusieurs de mes divisions les plus naturelles sont fondées d'après leur présence sur les hranches. Les spinosissima sont sépurés des canina par ce caractère. Le turbinata est séparé de ses alliés les plus proches paree qu'il n'en a point. Le R. histrix est séparé du reste de sa division parce que ses rameanx en sont pourvus. Ils sout sujets cependant à quelques anomalies dont on ne saurait rendre compte.

Le $R$. canina en fait voir quelques-unes dans sa tribu ou d'ailleurs on ne les rencontre pas ordinairement. Il elr est

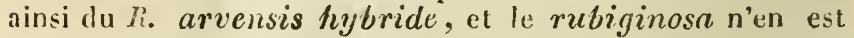
point exempt. Ces exceptions sont toutefois peu importantes. Elles ne doivent pas servir dans l'emploi du calactère des soies, pour le classement des espèces. Il faut remarquer qu'un organequi, sur les branches, est important pour l'observateur, est très-rariable sur contes les autres parties, notamment sur les fruits el pédoncules. Les $P$. rubiginosa et tomentosa produisent des fruits nus et soyeux, et quelquefois indifféremment sur la même tige. Le $R$. canina est souvent garni de soies sur ses pédoncules, rarement sur les fruits. Le spinusissima, le carolina et d'autres sont irgicment disposés d 
varier à l'égard de leurs pédoncules tantôt mus, tantôt soyeus. 11 y a pourtant des especees qui ne sont pas sujettes à de pareilles variations quant a la surface de leurstruits. l'ar exemple les R. involuta, Sabini, villosa, lavigata, sini$c a$, etc. , ront jamais élé trouvés inermes.

Les canina se distinguent facilement des villosa par la forme de leurs aignillons, et des rubiginosa par l'inégalité de ces mêmes aiguillons. Leur présence un lenr absence sur les pétioles est trop sujette it varier pour être considérée comme caractère constant ( 1 ) : lorique leur situation est infrastipulaire, connue dans la plus grande partie des cinnamomea, ils fournissent un caractère important; mais nous ne pouvons bien en juger que d'après des plantes vivantes, parce que la plus grande partie des especes pourvues d'ai- guillons épars, ne conservent souvent dans la dessiccation que ccux placés sous les stipules. Le fruit de quelques espèces est garni de soies ou petits airuillons persistans.

Jes glandes, qui se distinguent des poils principalement par leur odeur, sont pour l'ordinare attachées i la surface inférieure des léuilles. Elles servent à séparer les rubiginose des canina, le mynacantha du spinosissima, et notamment le R. brugnonii du moschata. Le R. pulverubita est le seul qui les porte à la surfice supéricure des leuilles. La substance remarquable, comnue sous le nom de mousse, el que l'on remarque sur le centifolia et le rubiginosa, peut être prise pour des glandes sous une forme particuliere.

La pubescence des branches, des péduncules, des fruits et des sépales est le seul caractère invariable que j'ile observé dans les rosiers. Je dois, avec raison, considérer comme certaines les distinctions que l'on en tire. Elle est ou persistante ou carduque. Sous le premier point de vue, elle devient un criterium important du setigera, et caractérise les berberifolia, ferox et bracteata. La premièr: des trois se distingute des autres par le défaut de pubescence sur le fruit. Quand elle est caduque elle devient seulement d'une importance spécifipue. Et sous ce denxieme point de vue, elle sert à distinguer le rosier abjssinica du sempervirens, le glutinosa du rubiginosa. Elle distingue partienlièrement le $R$. microcarpa dans la tribu des banksice. La circonstance de la

(r) Aussije ne le considère que comme caractère sous-spécifique, comme on verra dais la mnnoraphic. 
pubesccnce sur les feuilles est bien moins importante. Je in'en suis servi quelquefois, mais seulement comme un caractère sccondaice. J'ai ru des canina arosir des leuilles pubescentes et d'autres lisses. Dans cette espéce et dans le tomentosa, il y a une gradation complète d'une parfaite nudité à la pubesconce la plus épaisse. Le carotina a ses feuilles tonenteuses ou glahres; il en est de même du spinosissima, du semperforens, de l'arvensis et de plusicurs autres. Néanmoins, je n'ai jamais trouvé de duvet sur les feuilles du fraxinifolia, et celles du cinnamomea ne sont jamais parfaitement glabres.

Les stipules procédent de claque côté de la base du pétiole auquel elles sont plus ou inoins allhérentes. Certaines espéces, telles que le bracteata, l'involucrata, et parfois le canina, indiquent la nature de ces appendices en les produisant sous la forme de feuilles. Elles different de celles auxquilles elles sont attachées par leur petitesse et leur forme moins régulière. Leurs modifications ne sont pas nombreuses; mais quand elles ont lieu il faut s'y arrêter. Las série des banksiana est caractérisée par des stipules subulées, peu adhérentes au pétiole, et caduques comme dans le pêcher et le brugnonier. Les stipules pectinces du multiflora se distinguent parfaitement des especes arec lesquelles il a d'autres rapports. Elles sont étroites dans le majalis, larges dans le cinnamomea, aplaties et ondulées dans le lucida, contournées dans le carolina et le $W$ oodsii; elles ont unc direction constante dans le spinosissima, et brusquement divariๆ̨uées à l'extrémité dans le sulfurea. Dans le berberifotia, clles deviennent confluentes à l'exclusion des feuilles, et, leurs fonctions étant altérées, elles prement une texture plus ferme et plus rigide qu'à l'ordinaire.

Les feuilles sont toujours pinnées. Leur densité peut être quelquelois considérée comme dans le sempervirens, lequel, entre antres caractères, se distingue de l'arvensis par cetle particularité. Leur conleur mérite attention, si elle n'e pas due ì la pubescence. La teinte glauque du rubrifotia contribue à le distinguer du canina, et le taxa du tucida. La forme des folioles ne doit être employée que quand elle est trìs-remarquable; elles varient de l'ovale à- l'elliptique, de l'orbiculé à l'ovale; on remarquera que les formes lancéolíes et obtuses sont les plus constantes dans les folioles, et ce dernicr caractère suffirait pour séparer le $R$. sericea de l'espèce 
la plus voisine. La concavité des folioles n'est ordinairement d'aucune importance; cependant c'est un trait remarquable dans le $\boldsymbol{R}$. lutea, dont les folioles sont recourbées en cuillère, et confirment l'aflinité de cette espèce avec le rubiginosa, qui jouit de la même particularité (1). La rugosité des folioles fournira fréquemment de vrais caractères spécifiques. Elle distingue les villosa des canina, de l'acicularis et du reste de sa série, et le cinnamomea du majalis, Les folioles luisantes du $\boldsymbol{R}$. ferox ne ressemblent nullemeut aux folioles opaques du fantchatica. Les dentelures simples et duubles ne peuvent guère servir que dans des circonstances particulières; elles sont généralement trés-variables. Il est de certains canina pour lesquels l'oeil le plus exercé ne pourrait decirler si les fulioles sont dentées on surdentées. M. Woods assure que le myriacantha a de doubles dentelures. Dans tous mes échantillons ils sont simplement dentés. Une variété du mebiginosa, que $\mathbf{H}$. Wood appelle Borreri, a produit une année toutes les feuilles simplement dentées, et l'année suivante de surdentées. Cependant je crois que la double serrature de l'involuta empecchera qu'on ne le confonde avec le spinosissima.

Dans diverses espéces, la floraison varie de la forme la plus simple à la plus composie ; elle paraît conserver cependant un nombre assez régulier. Dans quelques séries, les fleurs sont solitaires comme dans les pimpinellifolice. C'est ce que l'on recounait par l'absence des bractées, dont la production concourt a l'auginentation des fleurs. Si dans une espèce bractéolée la fleur est solitaire, elle ne se trouve dans cet état que par l'avortement d'autres fleurs latérales. Le premier degré de la composition s'opère par une fleur munie d'une bractéc à sa base, et située sur un des côtés de la fleur primordiale. Quand l'inflorescence est augmentée, c'est encore par une semblable addition de bractées secondaires; en sorte que celles qui étaient latérales à l'égard de la fleur primordiale, deviennent centrales pour celles qui les accompagnent de nouveau. Ainsi la floraison doit toujuurs être considérée comme commençant par la fleur centrate, qui s'épanouit la première, et dont le pédoncule est plus court. C'est pourquoi l'on doit regarder comme rameaux toutes les ra-

(1) Je l'ai également remarquée sur plusieurs variétćs du li. alba. 
mifications sans fleurs, bicn qu'elles soient égaleraent sans fenilles.

Examinons, par exemple, le $R$. moschata dans son état le plus composé. La masse des ramifications se compose de ramilles alternes, pourvues ordinairement d'une feuille axillaire. Chacune de celles-ci produit d'autres ramilles altcrnes, la feuille infra-axillaire étant peut-être réduite à une seule divarication. Ces dernières sont encore divisées en nouvelles ramilles, et sielles sont arrivées au dernier état de composition, comme cela arrive ordinairement, chaque ramille est termiruée par une fleur sans bractée, et alors on peut dire que la floraisun est établie. La fleur primordiale s'épanouissant d'ahord, doit être la plus parfaite (I), et si l'ou veut en l'examinant s'issurer du nombre des ovaircs, qui est plus constant que dins les fleurs latérales, il ne faut pas confondre les premières a vec celles-ci, qui peurent devenir, accidentellement solititires et privées de bractées.

La formc des fruits (2) est un caraclère trop rarialsle pour être employée utilement: toutes les variations de forme se trouvent dans le canina el le tomentosa.

La forme des divisions calycinales (sépales) peut être quelqueiois considérée, mais rarement les degrés de division. Dans le Gracteata, elles sont larges, courles et pectinées; l'arvensis les a moins allongées qu'elles ne sont ordinairement. Néanmoins l'on doit toujours avoir égard à leur persistance. Elle distingue en quelque sorte le $W$ oodsiz du carolina, caractérise tous les pimpinellifutia, et fournit le principal diagnostique des villosa et canina. Leur réflexion contribue à séparer le damascena du centifotia et l'alba de quelques autres. Leur allongement est un trait marquant dans le rubrifolia, et doit être souvent employé pour distinguer de simples échantillons du cinnamomea de certaines variétés du tomentosa.

Les pétales offrent rarement des diffúrences notables. Ils

(I) La fleur centrale est plus forte et d'une meillenre forme, comme on peut le voir dans les centi/olia, damascena, etc.

(2) (Tube of the calyx.) Je répète encore que ie ne puis m'accoutumer à nommer tube un organe arrondi, renflé daus le milieu, ou ovoïde. Je sais bien que c'est le nom assigné par quelques auteurs à la partic inférieure dn calice; mais ici le calice devient fruit, et à une scule exception (le damascena) il n'est jamais tubiforme. Toutefois jc suis obligé de ne conformer à l'usage actuel. 
sont concaves et développés dans la plupart des espèces; refléchis intérieurement à leur extrémité dans le $R$. involuta, crispés dans le carolina, terminés en pointe dans le taurenceana.

Les ilamines varient seulement en nombre, et, sous ce rapport, elles doivent être rarement employées, sinon comme caractère secondaire. Par exemple, dans le rubrifolia et le reste de sa tribu, elles sont en trés-petit nombre. Dans les bracteate elles sont au contraire excessivement nombreuses: pour l'ordinaire elles restent adhérentes à l'orifice du fruit jusqu'i sa maturite. Hais dans le sempervirens, le moschata, le semperflorens, et quelques autres, elles tombent presqu'en même temps que les pétales. Cette particularité entre autres fait distinguer la dernière espèce de l'indica. Dans la première, elles sont communément environ quatre fois aussi nombreuses que les ovaires. Dans les spinosissima, reversa et indica, deux fois; dans les bracteata, ferox, acicularis, 5 fois, et $S$ fois aussi nombrenses dans le $R$. laurenceana.

L'ovaire peut, en certains cas, servir à faire distinguer les espèces; mais pour cela il est absolument nécessaire de les exanniner dans la neur primordiale. Les villosa en ont de 50 a 40 , ainsi que les rubiginosa. Les canina en ont de 15 à 25 , excepté le caucasea, dans lequel elles se trouvent de 50 à 60 ; dans le taurenceana ils se trouvent réduits à 7 .

Les styles par leur exscrtion et cohésion caractérisent une sćrie dont les espèces eonservent un rapport naturel. Il y a cependant une plante, le $h$. setigera, douée de la mêıne prarticularité et qui différe essentiellement sous d'autres rapports. Ses stipules subulées l'ont finit placer dans la tribu des banksianea.

a J'ai déjà dit plus haut ce que M. Lindley pensait sur l'importance attachée à la considération dı fruit. J'ai cru trouver dans ses divisions mêınes deux grindes sections qui les séparent en un nombre à peu près égal d'espèces. La plupart des auteurs, et récemment MII. Bose et Dumont Courset, ont adopté l'opinion de Linné á cet égard, et je ne fais ici que modifier les titres de leur's sections. *

Les espèces sont toutes renfermées entre le $70^{\circ}$. et le $20^{\circ}$. degré de latitude nord, excepté le $R$. Montezuma du Mexique, tronvé au $19^{\circ}$ degré nord, à la hanteur de plus de 9,300 
pieds au dessus du nircau de la mer (1). Mais M. de Humboldt a calculé que sous les tropiques la dininution de calorique est d'un degré par chaque go toises d'élévation verticale, en sorte qu'à cette hauteur la chaleur serait à peu près la même que celle des contrées situées à 29 degrés đe l'équateur; il suit de là que sa situation est essentielleınent la même que celle des principaux paralleles européens, et qu'il (le $R$. Montezuma) se rapproche plus de ses co-espèces européennes que de celles de son propre continent.

On a trouvé la moitié des espèces en Asie; des 39 qu'elle produit, 18 sont originaires des possessions russes et contrées adjacentes; la plupart de celles-ci. ont des rapports nombreux avec la partic européenne du genre; 5 d'entre elles sont communes à l'Europe et à l'Asie; quant au reste, une, qui est peut-être un genre distinct ( $\boldsymbol{R}$. berberifolia), a été découverte en Perse, 15 en Chine, et 2 de celles-ci avec 4 autres dans le nord de l'Inde. Une de celles-ci (du Népaul) (2) a de grands rapports avec le $R$. moschata de la partie septentrionale de l'alinique. Les espéces de la Chine et de l'Inde ont un port différent des autres, excepté le sericea et le macrophylla de Gossan, qui tiennent des uns et des autres an premier aspect. C'est de l'Asie, cette vaste contrée qui mérite le nom de terre des roses, que l'on doit attendre le plus grand nombre d'espèces nouvelles. Quant à celles de la Crimée, nous les connaissons peu, et l'on dit cependant qu'elles y croissent a vec une grande profusion. M. Nloor Craft a trouvé de petits buissons de roses à Niti, sous la latilude de $30^{\circ} 50^{\mathrm{m}}$. nord, qui commencaient seulement à montrer des fenilles le 9 de juin; il y a lieu de croire que la Chine produit encore d'autres espèces non décrites.

L'Europe possède 25 espèces dont les $\frac{5}{6}$ se trouvent entre le $40^{\circ}$. et le $50^{\circ}$. degrés. Les contrées rentermées daus ces limites doivent donc être considérées comme leur patrie naturelle. Dars les pays situés au midi de cette zone, flles paraissent en plus petit nombre que dans le nord. L'Angleterre, qui

(1) Il faut faire attention, si l'on voulait réduire en toises, qu'il s'agit ici de pieds anglais qui n'ont environ que 11 de nos pouces.

(2) Cette espèce, du Népaul, a éte rapportée par Olivier des jardins d'Hispahan, où elle croissait en arbre de la grosseur d'un poinmier. Cultivée pendant 7 à 8 ans dans une hâche, chez Cels, où elle a lleuri et fructifié, elle n'a jamais parı différer dı Rosa moschata (Bosc). 
se trouve juste dans les liusites septentrionales, en pusiede 10 , le Danemarck 7, et la Ilollande 13 espéces; landis que l'Espagne, le Portugal et le Levant, qui sont ì peu près à la même distance vers le sud, n'en offrent à l'observation que 4 espèces. Plusieurs sout particulières à eertinins districts, comme les reversa, myriacantha, hybernica el involuta; d'autres àdes contrées, comure le majalis de Suéde et de Danemarck, et le glutinosa du Levant. Un petit nombre est seulement confiné aux extrêmes limites de localité llu genre; ainsi le spinosissima est commun anx déserts glacés de l'Islande, et aux brûlans rivages de la Méditerranée. Le: canina crô̂t depuis les confins de l'Angrermanie jusqu'anx régions les plus méridionales de l'Europe, d'où il s'étend en Égypte.

Dans les régions septentrionales de l'Afrique deux espèces sont particulières à ces contrées et deux autres sont communes a vec l'Europe.

Quatorze espèces ont été trouvées dans l'Amérique septentrionale. Excepté le Montezumce et le stricta, elles ont une ressemblance générale avec quelques roses d'Europe (1). Il n'est pas hors de propos de dire que le $R$. Lavigata des lorêts de la Géorgie a tant de rapport avec le sinica de la Chine, qu’il n'est pas lacile de les distinguer. Ce derıier se vend da:ıs quelques pépinières de Londres comune un rosier d'Amérique, sous le nom de rose cherokensis.

* Nous cultivons dans les environs de Paris beaucoup de rosiers indigènes étrangers ¿i la France. Je crois qu'il est bon de remarquer que ces rosiers conservent dans notre climat une partie des habitudes de leur patrie. C'est ce que l'on peut remarquer dans la succession qui s'opère dans la chute des feuilles. Le $R$. ferox dı Caucase se dépouille le premier. Après lui vient le $\boldsymbol{R}$. alba originaire d'Italie. Le $R$. glutinosa du Levant; le sulfurea et le lutea suivent de près. Énsuite viennent les sinnumomea ef les rosiers américains, excepté le carolina florida qui est bifere. Les pimpinellifolia (2) se dégarnissent avant les cinnamomea, et dans le mêm: temps les centifolia, villosa, turbinata et rubiginosa, le damascena et l'espece suivante belgica perdent leurs feuilles par

(1) Notre Rosa cinnamomea renferme, dans sa série, la plus grande partie des roses ancéricaines.

(2) Les alpina, qui sont de cette série, se dépouillent en même temps. Le semperflorens les conserve par an hiver doux; mais il ne peut supporter 11 \& 12 degrès. 
la base ainsi que la variété portlandica, tandis que le gallica, qui les conserve plus long-temps que les autres, les perdà l'extrémité de ses rameaux; et une preuve qu'il faut séparer les pompons, c'est que le St.-François (gallica) les conserve aussi long-temps que ses co-variétés, tandis que les burgundiaca et variétés sont entièrement nus.» 


\section{ROSA.}

\section{PREMIERE SEGTION. - FRUIT'S GLOBULEUX ou OBRONDS.}

\section{PREMIÈRE TRIBU. - SIMPLICIFOLIAE.}

Folia simplicia, exstipulata (receptaculum impube, PaLlas ). Feuilles simples, sans stipules; réceptacle nu.

\section{PREMIERE ESPĖCE. - ROSA BERBERIFOLIA.}

R. Simplicifolia. S sllıs. Hort. aller. 35g. Parad. lond. 16r. c. figr. -Olivier. Voy. s. $4 \cdot 9$. all. t. 43.

R. Berberifolia. Geminibus globosis, peduneulis que aculeatis ; canle aculeis subgerminatis, uncinatis; foliis simplicibus; sulosessilibus. WILLd. Sp.pl. 1. p. 106.-P'ALL. in nov. Aet. petrop. 10.379. t. ro. f. 5. - Aix. Kew. ed. alt 3.25S. - Surtis iri Recs in 1.-liedout. Tos. 1. 27. t. 2.- Juss. Gen. 452 .

Se trouve en abondance près d'Amaden, son sol naturel. M1chacx. (Olivier.) Dans les chimps, an pird des monts Elvind. Olyvier. - Au désert de Sungarico. (Sievers). V. v. cult. in llort. Cels, Malmaison, etc. V.s s], iu Herl, gen. Il li.

(On ne connaît pas, du moins jusqu’à présent, de sous-espéces ni de variélés de ce Rosier).

Ce rosier, si différent de tinus les autres par son feuillage, a 2 ou 3 pieds de hauteur suivant quelques botanistes, mais seulement a pied d'élévation d'après Olivier, ce qui s'accorde avee les échantillons qu'il a rapportés et que j'ai rus dans l'Herbier général du Muséun d'Histoire naturelle. Il a une apparence bleuâtre; ses branches sont menues, et leur pubescence disparaît sur les ramilles. Aiguillons menus, arqués, presque décurrens à leur base, quelquefois 2 à 2 à la naissance des feuilles ou des ramilles. Feuilles sessiles, droites, simples, étroites, ovales, simplement dentées à l'extrémité, fortement duvelées, inermes; point de stipules. 
Ftaurs sulitaires, sins bractes scyphiformes (d'une odeur ducce, Olivier ). Fruit cotonneux, presque rond, et couvert jusqu'aux sépales d'aiguillons aeiculaires et inćgaux. Sépales cotonneuses et entieres. Pélales d'un jaune foncé avec une tache cramoisi-obscur à l'onglet. P'eu d'étamines. Styles velus. (Fruit eouronné par les sépales, et d'un vert clair, Pallas.) Ovaires, 25 , oblongs et noirûtres.

Quoique le nom que M. Salisbury a donné à eette belle plante ait été publié avant celui de Pallas, et qu'il soit plus convenable, comme l'observe Smith, toutefois celui de berberifolia ayant été universellement adopté, je ne eroirais pas pouvoir faire céder la convenance à la priorilé, qui d'ailleurs n'est soutenue que par un auteur peu important. L'ensemble de cette plante diffire du reste du genre d'une manière remarquable; ct réritablesnent l'absenee des stipules, qui ne peuvent être inétamorphosées en aiguillons, comme l'i conjecturé 11. de Jussieu, nous induirait à el établir un genre particulier, surtout si le réceptacle est dépourvu de poils, comme Pallas l'assure, et ce que nous n'avons encore pu vérifier. Peut-être aussi n'est-il pas impossible que la plante soit sans feuilles, en supposant que ce qui en porte le nom soit des stipules confluentes. C'est le seul rosier qui porte des aiguillons composés.

Des districts particuliers dans le nord de la Perse, et le désert de Songari dans la Tartarie chinoise, sont les seules contrées où l'on ait trouvé jusqu'à présent cette jolie plante. Olivier la vit couvrant les plaines prés d'Amadan, et dans plusieurs autres lieux du voisinage. Si nous pouvons juger de ses dimensions par la belle figure que ll. Riclouté en a donnée, les jardiniers français doivent avoir le talent de la ctiltiver beaucoup mieux que les nôtres (1). Peut-être la qualité salée du sol peut éclairer les amateurs sur sa culture. Elle fleurit au printemps.

(1) Les peintres en miniature doivent, pour réussir, faire ressembler en bean, et il arrive que l's peintres d'histoire naturelle, notamment des plantes, font ressembler en grand. L'exactitude des proportions est de rigueur à ce qu'il me semble. Curtis et Andrews ne sont pas plus exempts de ce reproche. (Note du traducterur.)

Le fait est vrai; mais le pied dessiné par Redonté au Jardin des Plantes, lequel était greffé sur un spinnsissima, était trois fois plus gros dans toutes ses parties que les échintillons apportés par Olivier ; j'en ai un exemplaire. Cela a lieu tontes les fois que l'on greffe une especce sur une plus forte plante en bon sol. (bosc.) 


\section{IIe. TRIBU. - FEROCES.}

Caractère particulier. - Rameaux et fruits revêtus d'un duvet peristant. Fruit nu. Les plantes pourvues de ce caractire forment une réunion d'espèces peu nombreuse, mais naturelle et très-saillante. Ce sont des arbrisseaux peu élevés, qui perdent leurs feuilles en automne, ef se font remarquer alors par l'aspect dénué de leurs branches cuuvertes de nombreux aiguillons. Leur fruit, qui est dépourvu de duvet, les distingue sans peine de la Iribu suivante dans laquelle la pubescence est trés-apparente.

\section{ESPËCE. - ROSA FEROX.}

R. Armis confertissimis, inæqualibus, conformibus.

R. Ferox. Lawr. Roses. t. 42.- Br. in Aiton Kew. ed. alt. 3. 262.S.мiтu in Rees in 1. - Lindu. in Edwards's. Reg. t. 420.

R. Kamtschutica. Redoote. Roses. 1. 47. t. I2.

IIab. in Caucasio. (Аıтом), V. v. cult.

4 ou 5 pieds de haut. Branches tomenteuses, inclinées, couvertes d'aiguillons inégaux, rigides, effilées, pâles, pubescentes ou sétigères. Feuilles brillantes, d'un vert éclatant et ridées. Stipules larges, dilatées à leur extrémité supérieure, tomenteuses, bordure marginale glanduleuse et contournée. Pétioles glanduleux et sétigères, portant des aiguillons jaunâtres, menus et presque droits. Folioles de 5 à 9 , elliptiques, dentées, presque point surdentées, lisses en dessus, velues et pâles en dessous. Fleurs rouges. larges et solitaires; sans bractées, ou, s'il y en a, elles sont larges, presque orbiculaires, et bordées de poils glanduleux. Pédoncules tomenteux. Sépales étroites, triangulaires, parfois un peu composées et duvetées. Pétales concaves, presque en cœur, ondulés. Élamines, 150-185. Disque un peu élevé. Ovaires, 50-60. Styles velus, séparés en dehors. Fruits d'une teinte écarlate très-fraîche. Partie supérieure du pédoncule nue. Péricarpe jaune et soyeux.

La rose hérisson (hedge hog), nom sous lequel le $\boldsymbol{R}$. ferox est connu dans les jardins, paraît avoir été publiée pour la première fois par miss Lawrence, qui l'avait probablement 
abtenue de la collection de M.M. Le et Kennedy; car ce sont ces laborieux cultivateurs qui l'ont fait connaître les premiers.

M. 'T hory l'a étrangement confondue avec le kamischatica qu'il considire coume ayant produit le ferox par la culture. Tout botaniste qui aura soigneusement observé les deux plantes vivantes, reconnaîtra que cette descendance est peu probahle; outre la différence entre les aiguillons, sur laquelle est fondé leur caractère spécifique, je puis ajouter que le liamtschatica est plus grand que le $R$. ferox; ses feuilles sont opaques, peu brillantes, plus petites, arec une différence d'aspect, changeant de couleur et tombant au commencement de l'automne arant que celles du $R$. ferox ne soient fances; son fruit est aussi plus petit et plus court que les sépales, qui ne paraissent pas disposées ì se composer. Dans le $R$. ferox, au contraire, clles sout plus sourent composées qu'autrement, et dans plis d'une circonstance j'ai obserré que deux d'entre elles élaient des feuilles parfaites. Les autres conservent la forme indiquée prar le vieux distique :

Quinque sumus fratres, etc.

Si la plante est tenue dans un état vigoureux en la taillant de près, elle deviendra très-remarq!able par ses fleurs purpurines qui paraissent arant des especes plus odorantes.

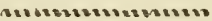

\section{ESPËCE. - ROSA RUGOSA.}

R. Armis confertissimis, subæqualibus, pedunculo aculeato. Lindo. Mon. p. 5. cum fig.

R. Rugosa. Thunb. Jap. p. 213.-Willd. Sp. 2. p. 10jo.-Pers. Syn. 2. 4S. - ऽмати in Rees in loc.

liamanas Japonorum. THUNв.

ll Vamanas? Icones japunens. in Bibl. Dasks.

Mab. In Japoniâ (Thone.).

Ce rosier n'est connu que par le rapport de Thunberg dont la description contient peu de caractères qui le distinguent du R. ferox et du kamischatica, Les habitans du Japon l'appellent ramanas au dire de Thunberg.

Dans la collection des dessins japonais de la Bibliothéque de S. Jos. Banks, un trouve un rusiur appelé Vamanas, qui se rapporte assez arec la description de Thunberg, et qui est probablement le même, ainsi que la ressemblance des noms semble l'indiquer. 
Jes branches sout uenues (enurertes de duvet, 'Th.). armées d'aiguillons très-rapprochés, droits et presqu'égaux (inégaux, Tlı.); point de stipules ; pétioles (tnmenteux, Th.) avec quelques aiguillons épars et rigides. Folioles ovales, rugueuses, simplement dentées (ohtuses et mucronées, duvetées en dessous, Th. ) ; les nervures très-serrées. Fleurs solitaires; point de bractées; pédoncules (duvetés, Th.), chargés d'aiguillons droits, courls, épais, verticillés, élargis a leur base. Calice (globuleux, Th.), olsrond et nu; sćpales entières, rúfléchies, convertes de poils (Th.); très-itroites. Deux d'entre elles ont l'extrémité foliacée et dentée. Pétioles émarrinés.

En supposant que cette plante soit celle de Thunber r, ce dont nous pouvons á peine douter, on la distinguera aisément de ses voisines par ses nombreuses folioles , par ses aiguillons presque égaux, et par les petits aiguillons droits et verticillés du pédoncule, dont la forme contraste évilemment avec ceux que l'on remarque d'ordinaire sur cette partie.

" Il parait certain qu'avant la figure donnée par Lindley, nous ne pouvions établir de différence entre le rugosa et le liamtschatica, puisque celui que nous prenions pour ce dernier est le ferox qui croît dans une rérion plus rapprochée de la nôtre."

\section{4․ US̈PËCE. - ROSA KAMTSCHATICA.}

R. Aculeis infra-stipularibus, falcatis, unajorihus; foliis opacis.

R. Kamtschatica. Vent. Cels. t. 67. - 11т. Kiew. ed. alt. $3.350 .-$ Pers. Syn. 2. 47. - Sutru in liees in loc. - Lisdery in Edward's Reg. t. 419 .

Hab. in Kamtschatcæ locis siccis sarosis. Nelson. ( $/$. v. cult. et s. sp. llerb. Baxks.)

Haute de 3 et 4 pieds, revêtue comme le R. fcrox; branches duveties, brun-pâle, inclinées, pubescentes, garnies de poils et d'aiguillons qui tombent souvent dans la vieillesse. Aiguillons stipulaires larges, courbés, placés de deux à trois ensemble; les intermédiaires plus courts. Feuilles grises, opaques. Stipules larges, dilatées à l'extrémité, ordinairement courertes de poils, contournées sur les borls et frangées de grlandes. Pétioles inermes et duvetés. Folioles 5 à 9, ovales, émoussées, simplement et profondément dentées, serratuses, 
calleuses à l'extrémité: les folioles sont nues en dessus, tomenteuses et pâles en dessous. Fleurs solitaires, d'un rouge foncé. Bractées elliptiques, presque nues. Pédoncules pourpres, velus à leur base. Calice globuleux et nu. Sépales très-étroites, duvetées, faiblement glanduleuses, un peu élargies à l'extrémité, plus longues que les pétales qui sont cordiformes et mucronés. Étamines 160 à 1 70 . Disque élevé, plus apparent que dans le R. ferox. Ovaires, 5o. Styles velus, séparés. Fruits sphériques, écarlates, plus petits que dans le ferox. Péricarpes petits, brillans et lisses.

On considere ce rosier comme nouvellement introduit dans les jardins de l'Europe; mais il élait connu par des descriptions vers la fin du dernier siècle. S. James Smith en possède un pied récolté au jardin de Chelséa en 1791; et l'on voit dans l'herbier de Linné des specimens avec des fruits, désignés sous le nom china, que je regarde comme la même plante. C'est cependant M. Ventenat qui l'a tait connaître plus particulièrement dans son Jardin de Cels. Elle fleurit dans la plus grande partie de l'été à des intervalleș peu réguliers. Les seuls exemplaires bien conservés que j'aie vus sont dans l'herbier de Banks : ils ont été recueillis par Nelson dans le dernier voyage du capitaine Cook. Ils différent de la plante cultivée par des fulioles ovales et plus nombreuses, par des fleurs plus petites, et par plus de dissemblance dans la forme des aiguillons.

\section{III'. TRIBU. - BRACTEATA}

Caractères particutiers.-Dans les plantes de cette série, les raneaux et les fruits sont revêtus d'un duret persistant. Elles se trouvent dans le continent de l'Asie, depuis le Népaul jusqu'en Chine, et elles se distinguent facilement des précédentes par le duvet fort épais de leur fruit, caractère qui leur est particulier. Les feuilles sont épaisses, ordinairement luisantes; les aiguillons placés deux à deux sous les stipules; les sous-espèces qui constituent cette séric peuvent être considérées comme présentant dans leurs organes de fructification le plus parfait développement du genre. Les étamines varient de 550 à 400 ; les ovaires, de 140 à 170 . Les premières sont deux fois et les derniers trois fois aussi nombreux que dans la 
série précédente, qui tient, selon toute apparence, le second rang dans l'échelle du développement.

\section{5'. ESPECE. - ROSA INVOLUCRATA. ( LINDL. morl. p. 8.)}

fi. Foliolis lanceolato-ellipticis infra-tomentosis; bracteis contiguis, pectinatis.

R. Inolucrila. Rопхв. Fl. ind. ined.

R. Palustris. Buchanani. MS.

Hab. in Nepalia (Buchanan), Bengalia, tempore fervido ineunte florifera, pluvioso fructifera. Roxв. MS. China, ic. Sinens. (V. v. cult. et s. spec in Herb. LaMBERT.)

Branches d'un brun pâle, flexihles et couvertes d'un duvet très-doux. Aiguillons généralement nus, à base élargie ou prolongée, d'un brun clair, placés par paires sous les stipulcs qui sont distinctes, soyeuses et divisées à leur limbe en plusieur's segmens capillaires composés, bordés de glandes éparses. Ces segmens sont plus courts sur les pousses vigoureuses; les parties dégargées se terminent alors par unc petite feuille pinnée. Pétioles faibles, soyeux, armés de quelques petits aiguillons. Folioles de 5 à 9 , elliptiques, lancéolées, obtuses, brusquement dentées, d'un vert nbscur, mais soyeuses, et plus pâles en dessous dans la partie supérieure (rarement nues). Fleurs blanch's, presque solitaires, entourées de 5 ou 4 feuilles rapprochées. Bractées pectinées, Iaineuses ainsi que les courts pédoncules. Tube du calice globuleux déployant des sépales entières; pétales émarginés, plus longs que le calice. Disque large et épais. Styles velus et un peu saillans.

"Je suis rederable à M. Lambert d'avoir pu examiner des échantillons de cette nouvelle espèce : ils ont èté recueillis dans le Népaul par le docteur Buchanan qui les a trouvés dans des marécnges, quoique le docteur Roxburgh n'en fasse pas mention dans son manuscrit dı Flora indica, où il donne des détails sur cette espece dont il conserve la dénomination. Elle a été importée depuis pen des Grandes-Indes par Mil. Whilley et compagnie, de Fulham, dans la betle collection desquels je l'ai vue croître vigoureusement : elle me parait digne de trouver place dans nos jardins. Elle ne pourrait être confondue qu'arec le $R$. bracteata, duquel ses 
feuilles étroites et de peu d'apparencé, et gercẻes en dessous (pruinosis) la distinguent suffisamment. En outre, ces bractées sont notablement distantes des fleurs. Une figure prise dans une collection de dessins chinois qui appartient à M. Cattley, prouve que cette plante se trouve a la Chine comme dans l'Inde.

De pense, comme M. Lindley, que le Rosa clinophylla de 1 . Thory (Red., Roses 1, 45, t. 10, et Thory, Prodr., p. 126) ne saurait être le même que notre involucrata. La description présente des différences remarquables."

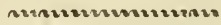

\section{ESPËCE. - ROSA BRACTEATA.}

R. Foliolis oblongis, obtusis, glaberrimis; bracteis appressis, pectinatis LINDL. Mhn. p. 10.

R. Bracterta. WeMdr. Obs. p. 5o. Hort. herrenhus, 7.t. 22-Vent. Cels.t 28. - Renout. Fios. т.35. t. 6. - Brow. Nom. p. 106.

R. Lucida. Lous. Rios. t. 8\}.

R. Macamueu. Den. Couns. Bot. cult. fide. - Pedout. idem.

Var. f. Scabriusculis, ranis setigeris; aculeis minonibus, rectiusculis. Linde. loc. cit.

R. Bracteata. Moencu. Meth. Suppl. 29o. - JacQ. Fragm. 30 t. 34.

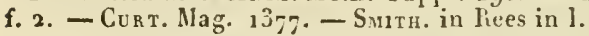

Hab. in Foutan, hoxв. (Var. $\beta$ in Chine provincia Tchetkiang, Staunton. (V. v. cult. et s. sp.)

Arbuste touffu, d'un vert foncé. Branches droites, fortes, robustes et cotonneuses. Aiguillons courbès en bameçn, très-forts, un peu soyeux et placés pur paires sous les stipules. Siipules presque distinctes, velues, pectinées; segmens capillaires; la partie supérieure dilatée, quelquefois en folinles pinnées. Pétioles presque nus, armés de quelques petits aiguillons forts et crochus. Folioles, de 5 à 9 , crinelées, presque ovales, aplaties, luisantes, nues des 2 côtés, d'un vert foncé en dessus, plus pâles en dessous ; nerrures peu apparentes. Fteurs éclatantes d'un blanc pur, solitaires, presque sessiles au milicu de plusieurs bractées ovales, imbriquées, soyeuses, finement pectinées en leurs bords. Tube du calice et sípales presque simples, laineux extérieurement. Pétales grands, presque ovales. Disque fort épais, aplati. Etamines, 550 à 400 . Ovaires, 140 à 170. Styles distincts, nus. Fruit splérique, rouge orangè, entièrement laineux. Púricarpes brunatres, ridès, enfoncés dans l'énorme quantiti de poils du réceptacle. 
Ce bean rosier, quoique indigène $₫$ la Chine et dans les provinces septentrionales de l'Inde, supporte très-bien nos hivers ordinaires, et produit en abondance ses fleurs d'un blanc de lait pendant la plus granile partie de l'été. fruit.

M. Lindley doit à M. Lyell l'avantage d'en avoir étudié le

Il a de fortes raisons pour croire que la variété $\beta$ pourrait, it l'aide de nouvelles observations, constituer une espice distincle. En effet, elle est beancoup p!us petite; clle forme un buisson tris-serré; les aiguillons sunt plus laibles et plus droits, et la tige est soyeuse, ce qui n’a pas lieu dans l'espèce proprement dite. 'oute cette tribu est particulière au continent de l'Asie, où elle croît à des degrés de latitude assez rapprochés. C'est à lord Hacartney, anbassadeur en Chine, que nous derons l'acquisition de cette belle plante.

\section{ESPE்CE. - ROSA' LYELLII.}

R. Foliolis ohlongis, lanceolatis, glabris; bracteis distanlibus, integris; floribus cymosis. Linde. Mon. p. 12. f. 2.

Hab. in Nepalit. W aldich (v. s. sp. Ilerb. Banкs). Amicissimo Carolo Lyellio, ann. botanices indigen $x$, præcipuè cryptogamicæ pcritissimo, susceptique nostri fautori accrimo, dicata.

Petit arbuste ayant l'apparence d'un bracteata. Branches fortement velues, sans soies (poils rudes). Aiguillons droits, placés par paires sous les stipules. Fcuilles épaisses, étalées, plus longues que les articulations de la tige. Stipules velues, adhérentes, divisées à leur limbe en plusienrs petits seginens étroits, un pell frangés de glanc'es. Petioles colonneux, armés de quelques airuillons crochus. Folioles 7, oblongues, lancéolées, très-luisantes, nues de's delx côtés, excepté la nervure principale qui est cotonneuse; dentelures simples. Fleurs en cime. Bractées distantes du calice, linéaires, Iroites, grises, entières. Pédicctles givrés, allongés, glaniluleux. Tube du calice et Sépales presque simples, plus courls que les pétales, cotonneux. Les pétales et autres parties de la fructification, paraissent être les mêmes que dans le bractcata.

J'ai saisi arec plaisir l'occasion de dédier une aussi belle espèce à mon exccllent aıni M. Lyell, dont les connaissances 
étendues dans cette partie et sa complaisance à les communiquer méritent cet hommaŕ à tant de titres.

Elle a été envoyée depuis peu du Népaul, arec une grande quantite de plantes intéressantes, par le $\mathrm{D}^{\mathrm{r}}$. Wallich; ses bracties étroites et entières, situées à une distance notable des fleurs, la font distinguer au premier coup d'œil du reste de la tribu.

\section{IV . TRIBU. - CINNAMOMEAE.}

Caractères particuliers.-Setigeræ, Y. inermes, bracteatæ. Foliola lanceolata, glandulosa. Discus tenuis (nequaquan incrassatos ). Les rosa alpina el acicularis de la tribu suivante ont aussi des bractées, mais les sépales tombent arant la maturité du fruit.

Cette tribu se distingue particulièrement par ses feuilles Inngues, lanceolćes et dépourvues de glandes; ses rejetons droits (surculi); un aspect dense (compacte); ses fleurs rouges, jamais solitaires, sinon par a vortement, souvent corymbifères et toujours pourvues de bractées; un disque presqu'imperceptible, mais un peu épais; un fruit rouge, petit, rond, perdant ses divisions calicinales ( sepala) après la maturité; un petit péricarpe lisse el luisant; les rejetons ordinairement soyeux (setigeræ) à leur base, rarement à leur extrémité, si ce n'est dans une ou deux espèces; tous ces caractères concourent à distinguer suffisamment celte tribu de ses voisines.

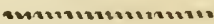

\section{8* LSPÈCE. - ROSA NITIDA.}

R. Pumila. $\Lambda$ rmis confertissimis, gracilibus; foliis nitidis, angustè Janceolatis, planis. LINDL. Mon. p. I3. f. 3.

R. Nitida. Wirld. Enum. $54^{\prime}$.- Pursh. Am. septr. 1. n. 3. - Sмгтн in Rees in 1. - Poir. Enc. suppl. $7 \mathrm{~J} 1$.

12. Rubrispina. Bosc. Dict. d'agr. p. 246.

R. Blanda. Pursh. l. c. n. т. et in suppl.

R. Redulea rubescens. Redout. Ros. I. Io3. t. 36.

Hab. in Terrâ-Novâ. Herb. BAvcks. (V. v. c. et s. sp.)

Buisson bas et rougeâtre. Branches droites, très-divisées, toutes couvertes d'aiguillons faibles, dissemblables et entreurêlés de soies. Les feuilles très-claires ou luisantes, d'un ver't 
foncé, prenant unc conleur jourpre en antomne. Stiputes splaties, nues, frangées de glandes, entières ou peu denties, ovales a leur cxtrémité. Pétioles faibles, nas. Fotioles le 3 d̀ 7 , étroites, lancéolées', nues; dentelures simples; nervures peu remarquables. Cimes en corymbes panciflores. Bractées lisses, ovales, lancéolées, ondulécs, contournées. Pédoncules couverts de soies presque égales. Tubes du calice soyeux et presque sphérique. Sépates étroites, plus courles unue les pétales, saus poils ni duret. Pétales presqu'en cœur, tiès-ronges et brillans, concaves et presque droils. Étamines 100 à 150 . Disque épais et aplati. Ovaires 50 ì 55 . Styles distincts, velus, renfermés. Fruic écarlate, brillaut, sphérique, romprimé, un peu hispide.

Jolie petite espèce d'un rouge éclatant. Ses fleurs, en forme de coupe, different prodigieusement du $R$. btanda avec lefuucl Pursh l'a conlondue mal ì propos; car le fruit, d'après l'inspection du sujet qui se trouve chez M. Sabine, prouve cuil a changé le caractére du blanda dans son supplément. II est possible qu'il ait eu une autre plante en vue en faisant son nitida; mais en cela même rien ne peut le justifier. On l'appelle communément dans les jardins la Rosenaine du LaGrador. La $27^{\circ}$. fig. de $\mathrm{H}^{1 \mathrm{ll}}$. Lawrence paraît en être une mauvaise copie, et cependant le savant auteur de la monographie de l'Encyclopédie de Rees l'attribue au blanda, suivant la seconde édition de l'Hortus kezvensis. Quant au R. rubispiuct de M. Bosc, j'hésite à le rapporter comme synonyus; mais le $R$. redutea rubescens de Thory est certainement notre plante. Quelle est la différence qui existe entre celleci et le type du redutea? C'est ce que je ne puis découvrir.

Jecultive un rosier de M. Noisette, provenant de la collection de Dupont, qui me paraît avoir de grands rapports avec le R. nitida.

\section{9 . ESPÈCE. ROSA RAPA. (Bosc.)}

1.. Elatior, diffusa. Ramis inermibus ; foliolis olslongis, undulalis, lucidis; fructu hemispherico. Linol. Mon. p. 13.

R. Rapa. Bosc. Dict. d'agr.-Desf. Cat. hort. par. 273. - Poir. Cul. suppl. p. 7 10. - Pidout. Koses. 1. 7. t. 2. - Phonv. Num. 27.

R. Turgida. Pers. Syn. 2. 49.

R. Fraxinifolia. Doir. Coors. Bot. cult. fide Poiret.

Var. B. Hudsoviana. Kamis strictis; floribus corymbosis, numerosis. R. Hudsoniana. ThоR. Prodr. p. 62. 
Hab. in Americæe septentrionalis provinciis calidioribus. Frasrn. ( $V$. v. cult. et s. sp.Var. ß. baie d'Hudson. Thок.)

Arbuste plus élevé que le $R$. blanda, mais d'un port moins touffu; sans soies, ou bien un petil nombre d'aiguillons sétiformes, pâles, dégénérant en poils rudos. Rejets très-rouges, couverts d'aiguillonset de soies cramuisics, inégaux et épars, dont les plus grands sont courbés et comprimés. A mesure qu'ils diminuent de dimension, ils deviennent plus droits et finissent par se changer en soies. Feailles distantcs, teintes de rouge, plus colorées en automne. Stiputes nues, aplaties, ondulées ou étroites ou très-dilatécs, fimement dentées. Pétioles armés d'un petit nombre d'aiguillons courts et druits, eutremêlés de glandes. Folioles de 5 à 9 , à dentelure simple ou double, ondulies sans aucune pubescence. Cimes portaut plusicurs fleurs surmontées par plusieurs pousseses. Bractécs orales, lancéolées avec une pointe, longues et ouvertı:s, mais finement dentées. Pédoncules rudes à cause des soies et des glandes. Tube dı calice en coupe, aussi scabre à soll extrémité que les jédoncules. Sépales composées, terminées par une pointe plus lougue que les pétales, hispides en dehors. Pétales doubles, d'un rouge chair, plus petits que ceux du R. lucida. Disque presque oblitéré. Fruit rouge foncé, courohimé de sépales réfléchics, rond, avec un très-grand réceptacle rempli de styles velus.

Très-luclle espèce à cause de ses fleurs doubles et nombreuses. Le premier naturaliste qui en a fait mention est M. Bosc, dans le Dictionuaire d'agriculture, dont il est le principal auteur. Mais M. Bosc n'a point dit, du moins dans son dictionnaire, que ce rosier l'at originaire de l'Écosse, et si cette erreur a ćté copiée depuis peu des auteurs français, elte n'est point de son fait. La figure donuée par M. Redouté est d'une couleur plus verte que je ne l'ai vue mulle part. Je possède des échantillons recueillis dans les parties inćridionales des Etats-Unis par $\mathbf{M}$. James Fraser, et je suis redevable à 11. Robert Swect de très-beaux fruits qu'il produit rarement. Il y a long-temps que je connais cette plante, et je ne puis la considèrer comme variété du tucida; mais ils doivent être placés a la suite l'un de l'autre, dans une disposition naturelle du ginr'. Le Rosa lucida est un arbuste très-louffu, avec des feuilles nounbreuses et rapprochées, et des aiguillons sous les stipules; les l'umeaux sont corymbifères à leur extrémité; le 
réceptacle petit : tous caractères qui ne se retrouvent point dans le $R$. rapa.

La variété $B$. hudsoniana porte des rameaux plus effilés; ils sont lerminés par des corymbes de fleurs duubles, d'un rose pâle, plus nombreuses quc dans l'espece, mais n'ouvrant pas toujours bien. (étte varif́té, d'un port assiz gracieux à cause de ses rameaux réfléclise, a été dédice au célèbre royageur Hudson. Elle croît, selon 11. Thory, dans la haie qui porte son nom; mais je n'en ai aucune ccrtitude.

\section{ESPĖCE. - ROSA LUCIDA.}

R. Compacta. Aculeis rauulorum stipularibus; foliolis oblongis, inhricatis, planis, lucidis; fructu depresso-globoso. Lindu. Wion. 17.

R. Carolina. Fragans; foliis mediotenus serratis. Dibl. Elth. 325. t. 2;5. f 3,6 .

R. Rubra lucida. Roess. Ros. t. 7. et 1. 25. f. ז.

R. Lucida. Евн. Beitr. 4. 22.-WilLd. Sp. 2. 1068.-Mornsch. Meth. 687. - JacQ. Fragm. 71. t. 107. f. 3. - Pers. Syn. 2. 48.-Punsu. $\Lambda$ m. sept. n. 4. - Smiтn. in Rees in 1.- Redoute. Ros. 1.45. t. 11. - p Ronv. Nom. p. 26.

$H a b$. in Americ septentrionali, à Noveboraco in Carolinam usque (PURsh.); juxta Boston in æuquoreis et ad margiaes paludum (BIGELow.) ( $V$. v. c. et s. sp.)

Buisson ramassé, haut de 4 à six pieds, quelquefois moins. Branches droites, brun - rougeâtre, luisantes, a vec quelques aiguillons faibles sous les stipules, quelques soies assez rares. Rejets quelquefois très-soyeux dans la inoitié de leur partie inférienre. Feuilles très-serrées, se développant irrégulièrement. Stiputes sans pubescence, plates, luisantes, droitcs, créneléus, ayant leurs extrémités finement dentées ; dents terminées quelquefois par une glande. Pétiotes nus ou peu cotonneux par dessous, armés d'aiguillons forts et triscourts. Folioles 9, ovales lanceolées, nues des deux côtés, très-rapprochées, ondulées; dentelure simple et grossière; la paire inférieure souvent très-petite. Fleurs surm ontées par les feuilles et les nouvelles pousses, très-reuges, corymbifúres. Bractées concaves, contournées en leurs bords, ovales lancéolées aiguës, nues des deux parts, finement dentées, ou hordées de glandes pédicellées. Pédoncules presque, uns, pas plus longs ijue le fruit. Tube du calice globuleux et comprimé, couvert de soies (1). Sépales simples, ovales avec

"( 1 ) J'ai remarqué que, dans les cnvirons de Paris, les calices du 
une longue pointe, couvertes de soies dans la partie antérieure. Pétates presquu'orales, en cœur, un peu plus larges que les sépales. Disque aplati, peu épais. Riéceptacte éle vé au centre. Styles très-velus mais peu saillans. Fruit globuleux, compriıé, d'un rouge clair.

Celte espece n'est pas rare dans lés jardins, où l'on voit ses belles fleurs it la fin de l'été. Les différences qui existent entre elle et la précédente ont déjia été indiquées. Ses feuilles luisantes la distinguent tant du $R$. carolina que du $R$. taxa. Le savant président de la société Linncenne de Londres n’a probablement pas bien connu notre plante, car il n'aurait pas désavoué sa conformité avec la figure de Dillenius qui est trìs-exacte, et il n'aurait pas cité le $R$.alpina de miss Law rence, talb. -5 , var. 5 d'Aiton, qui est sans contredit le $R$. blanda de Jacquin, et non lı: R. Fraxinifotia. Cependant il a dans son Herbier de beaux spécimens indigènes recueillis par Bigelow ; et, i en juger par l'étiquette, il parait que l'espèce est commune dans les marais de l'Amérique septentrionale.

\section{$11^{\circ}$ ESPËCE. - ROSA LAXA.}

R. Diffusa. Ramis vimineis, subinermibus; foliolis oblongis, undu. latis, opacis, glaucescentibus. Linot. Mon. p. 18. t. 4

R. Carolina. E. A 1 r. Kew. ed. alt. 3. 260.

f. Carolina pimpinellifolia. ANDREws's Roses?

$H a b$. in Americâ septentrionali. ( $V$. v. cult.)

Arbuste étalé. Branches rondes, d'un brun rougeâtre, luisantes; aiguillons assez droits; rameaux privés d'aiguillons; les jeunes pousses portent dans leur partie inférieure de courts et faibles aiguillons, avec quelques soies. Feuilles non luisantes, rapprochées. Stipules étroites, s'élargissaut au sommet glanduleux. Pétioles velus, d'un vert rougeâtre, garnis de poils, de glandes et de faibles aigıillons. Foliotes de 7 à 9 , elliptiques, lancéolées, glauques, nues, onduices; nervures peu saillantes. Fleurs roses, ordinairement 2 ensemble. Bractées ovales et frangées, donbles nues. Pédoncules glanduleux. Tube du calice sphérique, garni de quelques poils. Sépalestriangulaires, lancéolées, presque entières ou pè denlées à leur extrémité, plus courtesque les pétales, velues, glan-

lucida et de quelques autres Rosicrs américains étaient rarement couverts de soies ou de glandes pédicellées." 
dululises et soyeuses antirieurement, particulièrement is la base. Pétales aplatis; disque oblitéré. Fruit inconnu.

Il est souvent cultivé sous le nom de Rosier de Caroline étalk. Ce n'est cependant pas a vec le $R$. carolina qu'on peut le confondre, puisque son port, ses feuilles glauques, ses stipules ouvertes l'en distinguent toujours. Le $R$. lucida en approche bien davantage. Il en differe sous les rapports suivans de manière i ne pas s'y tromper: A peine un aiguillon se voit sur les rejets; les rameaux sont faibles, souvent inermes, et toujours italés; les feuilles jamais luisantes portent une teinte gliurque. Il ne porte jamais de fruit, tandis que le luciula en lournit abondamment. L'époque de la floraison est différente, car le laxa fleurit au commencement de l'été. Je n'ai jamais vu de spécimen indigène, mais on n'élève aucun doute sur son pays natal. Il n'est pas sar que le $R$. carolina étale de malemois"lle Lawrence snit le même que celui-ci.

\section{2". ESPËCE. - ROSA PARVIFOLLA.}

R. pumila. Stipulis linearibus; aculeis acicularibus; foliolis lanceolatis, glabriusculis, argutè cerratis; calycibus viscosis. LiNDLEY. Mon. p. 20.

t. 25 ?

R. Carolina. Duros. Harbk. 2. 35\%. - S.M. Insects of Georgia. Y. 49.

R. Hunilis. MI ARSR. Arb. 136.

R. Parviflora. Eur. Beitr. 4. 21.-W1Lld. Sp. 2. 1068 . - Pens. Syn. 2. $48 .-P_{\text {Unsh. }} \Lambda \mathrm{m}$. sept. n. 2.-Sмiтh. in Rees in loc.-Pkonv. Nom. p. 25 .

R. Caroliniana. Mschaux. Borealis Am. 1. 293. The Pensylvanian rose. $-\mathrm{L}_{\Delta \mathrm{W}}$. Ros. t. 3 , et 66 .

R. Carolina b et d. A 1 T. Kew ed alt. 3. 260

Hab. in collium declivibus Novehoraco Carolinæ. Punsn. $r$. cult.)

Espèce basse, faible et étalée. Rejets couverts de quelques poils éphémères. Branches faibles, d'un brun rougeâtre, ar. mées d'une paire d'aiguillons aciculaires sous les stiputes; celles-ci tout-i-fait nues, très-étroites, un peu courbées, leur extrémité est plate et divariquée. Pétioles nus; folicies ordinairement 5 , un peu luisantes, lancéolées, pointues, fueinent et simplement dentées; nervures peu apparentes, celle du milieu un peu velıe. Flcurs carnées, jâles, ordiaairement par 2. Bracties orales, en cour, ponctures, unp eu velucs. Pédoncules couverts de glandes piulitlés, ainsi 
que le tube ilu cialice, qui est rond, petit et déprimé. Sepales ovales avec unc pointe aiguë, presque simples; horls cotonneux, visqueux : et glanduleux. Pétales Irès-nombreux dans la variété double, qui est la séule que j'aie vue.

La rose double de Pensylvanie est une des plus jolies que l'on puisse compter parmi celles de l'Amérique, et le céde i peine aux plus brillantes variétés du gallica; ses fleurs élégantes, à demi ouvertes, du rose le plus délicat, sa forme naine et tonflue, l'ont l'ait généralement rechercher, malgré les difficultés de sa culture et spécialement de sa propagation. Je crois que le terrain de brnyère lni eonrient parfaitement. Ehrhart, avec son exactitude ordinaire, est le premier qui la fit distinguer dı carolina et du tucida. Les observations de Mullenberg sur la plante qui se trouve dans l'herbier de M. James Smith me paraissent appartenir au tucida avec d'autant plus de raison, que ce dernier auteur cite les figures de mademoiselle Lawrence: sous le nom de $R$. carolina, ce qui n'anrait pas eu lieu s'il avait observi la véritable plante. Et cependant le $R$. carolina de Smith (Insectes de la Géorgie ) doit être celui-ci vraisemblablement, car la citation du pays natal est la même que dans l'Encyclopidie de Fiees. Je suis redevable ì 11 . Achille Richard d'une description détaillée du $R$. caroliniana de Michaux, qui m'engage a le rapporter au parviflora. On voit dans la collection de M. Lambert un spicimen de nos jardins ayant les feuilles presque linéaires.

\section{ESPECE. - ROS A WODSII.}

R. Stipulis sepalisque conniventibus; foliolis oblongis, obtusis, glabris. Lispl. Mon. p. 21 .

R. Luteo-nigra. Рhonv. Nom. p. 24.

$H a b$. juxta flumen Missouri. America septentrionalis. ( $V$. v. cult. HERIT. SABINE.)

Arbuste peu élevé, perpendiculaire, peu remarquable. Branches rembrunies. Aiguillons très-nombreux, droits, laibles, épars, garnis de quelques poils, souvent stipulaires à l'extrémité des branches. Rameaux souvent sans aiguillons ct inermes. Feuilles sans pubescence. Stipules trèsètroites, aiguës, contournées et frangées de glandes. Pétioles armés d'aiguillons visqueux. Fotioles de $\eta$ a 9 , de même que 
dans li. rubellu, luisantes, plates, simplement dentées. plus pâles en dessous. Flcurs roses au printemps. Fruils nus, ovales, a vec des sépales courtes, conniventes, entièrement privées le glandes ainsi que les pérloncules.

Quoique l'un puisse trouver parmi les rosiers nouvellement découverts en Angleterre une espèce digne de porter le nom de M. Wond, Jont j'ai déjá eu l'occasion de faire connaître le mérite, nous avons fait choix de cette espèce américaine, M. Sabine et moi, pour lui en faire hommage. Mlalgré les détails incomplets que j'ai pu en donner, il est évident qu'elle se: distingue de toutes celles de la même tribu. Je l'ai vue cultive pu:ı la jremière fois dans les jardins de M. Sabine, a Northmimms, dans une saison tardive. Dípouillé de ses feuilles, il rappelle le cinnamomea; mais, par ses caractères particuliers, il se rapproche du carolina, surtout i vause de la convolution de ses stipules. Ses nombreuses ranifications, ses aiguillons faibles, ses folioles courtes et luisantes, le distinguent aussi des autres espèces voisines.

Ln outre, il fleurit au printemps. Son fruit est nu et les sépales conniventes. M. Sabine assure que e'est cette plante qui a été envoyée en France d'une de nos pépinières comme un nouveau rosier d'Amérique, portant des fleurs noires et jaunes, et cité sous ce rapport, dit M. Lindley, dans l'ouvrage de 11 . de Pronville. On le croit indigène du Missouri.

"C'est à M. Noisette que je dois la première connaissance du $R$. W Woodsii. Il å fleuri depuis à Versailles, dans la collection de $\mathbf{1 1}$. Vallée, et j'en possède un pied qui pousse vigoureusement, et que je tiens de $\mathrm{HI}$. Sabine: il a fleuri chez uoi l'anuée dernière $(1823)$.»

\section{ESPECE. - ROSA CAROLINA.}

R. Stipulis convolutis; foliolis lanceolatis; sepalis patentibus. LiNd. Mon. p. $23 . t .5$

R. Carolince. Hem. Sp. 6o3. -Wuzd. Sp. 2. 1069-Lam. Ros. t. 3亿? - A $_{1 \mathrm{~T}}$ Kew. ed. alt. 3. 260. - Pers. Syn. 2. 48. - Porsu. Am. sept. wo. 8. - S.ritu. in hees in loc. - Redout. Kos. 1. 81, t. $2 S$.

R. Virginiana. Dogor. Harbk. 2. 353. - Roessig. lios. t. 13.

R. Palustris. Moensch. Arb. I53. - Doos. Cant. ed. 8. p. 169.

R. Curymlosa. Enr. Beitr. 4. 21.-Monn. Cat. 5o.

R. Pensyluanica. Mich А хx. Borealis Am. 1. 295.

R. Curoliniana. B. 29. Bost. 121.

R. Hudsoniana. Revovt. Ros. $1.9 \%$ t. 35. 
Var. B. Florios; foliis impubibus, tenerioribus.

R. Floricla. Doox. Cant. ed. 8. 169 .

R. Enneophilla. Rafn. Schm. Précis des découvertes.-QoodER. í - Desv. Sourn. L. 268.

Var. C. Genula. Ramis debilibus; floribus geminatis.

R. Gemilla. Wır d. Enum. 5.44. - Poir. Enc. suppl. 711.-(Voyez pour les détails p. 204.)

Hal, in palustribus Novi-Angliæ, Virginiam usque. Porsh. ( $V . \nabla$ : cult. et s. sp.)

Il s'élìve de 2 a 8 pieds (1); ses tiges sont droites, vertes ou d'uu rouge obscur. Aiguillons solitairi's ou géminc's sous les stipules; les aiguilions des rejetons sont plus serrés et peuvent être considérés comme des soies (2). Feuilles opaques. Stipules très-longues, étroites, réfléchies et même contournées, sinon à l'cxtrémité, qui est étalée, nue, dentée et liangée quelquefois en ses bords. Pétioles cotonneux, garnis de petits aiguillons. Folioles $\eta$, lancéolees, finement el simplement dentées, nues, d'un vert foncé en dessus, cotonniuses, un peu glauques par dessous, caduques vers l'automne. Cimes composćes d'une ou plusieurs fleurs apparaissant après les chaleurs de l'élé. Bractées lancérilées, très-concaves, pointues, cotonneuses en delors. Pédoncules et calice entièrement hispides; ce dernier est sphérique et d'un vert coloré. Sépates entières, a vec une pointe longue et cotonneuse en ses bords. Pétules concaves et aplatis, ordinairement plus larges que les sépales, d'un ronge foncé, ondulés. Disque peı apparent. Styles velus. Fruit écarlate, rond, hispide. ne perdant les sépales qu'il sa parlaite maturité.

Les massils d'arbustes sont tonjonrs embellis par ce rosier, lorsque l'on distinguc ses cimes nombreuses au milieu des autres fleurs. Dans les marais, où il est indiggene, il est sujet ì varier en bauteur, en forme, dans la pubesceace des feuilles et dans le nombre des fleurs. Il n'est pas moins disposé í varier par la culture; sa dimension moyenue est de 6 pieds. Ordinairement les pédoncules sont courts ; mais, lorsqu'ils sont nombreux et allongés, ce Rosier devient le corymbosi d'Ehrharl; si ses dimensious sont plus grandes et ses rejetons plus jûtes, c'est le Rosa palustris. Dans la variété $\odot$, qui est de basse tige, a les rameanx amincis et les corymbes peu nom-

(1) Je ne l'ai jamais vu, aux environs de Paris, atteindre au delá de cinc pieds.

(2) Cette disposition des rejetnns est commune à un grand nombrc d'especes, mais surtout anx $f^{e}$. et 5 e. tribus. 
breuk en lieurs, mais qui est bifere, on rutronve le $R$. virginica de nos pépinières. Une augnentation de pubescence l'a fait conlondre avec le pensylvanica; et la même espéce trisluxuriante et dipourvac d'aiguillons dans ses rejetons a cite confondue avec le $R$. hudsoniana.

"Li'on voit par ces détails, comme je l'avais déjia dit dans ma Nomenclature, que le $R$. carolina était fort difficile a détermincr à cause de ses variations, et que M. Lindley a trés-ljien fait de les réunir dans le mêne fuisceau." .

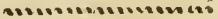 \\ $15 \circ$ ESPECE. - ROSA BLANDA.}

R. Elatior. Armis deciduis; foliolis oblongis, planis; petiolo pilusu. LiNnL. Mon. p. 25.

R. Blanda. B. Solander. MS.

1. Blanda. Aiт. Kew. 2. 202.-WVilud. Sp. 2. 1065.-Surtu. in hees in 1.-Provr. Nom. p. 19. - lal. Somm, p. s'́ .

Se trouve dans l'Amérique septentrionale, à Menzies, baie d'll udson. ( $V$. s. Ilerb. Bavks et Smith. (Lindex) et à l'tlerbier général du Jardin-Royal, ì Puris.)

Rameaux armés de soie; et d'aignillons énars, pâles, inçaux, caducs et droits. Feuilles de peu (Tapparence. Stipules longues, elliptiques, arrondies a leur extrimité et frangées de glandes. Tige cotonneuse, sans aiguillons. Folioles $5-7$, lancéolées ou plus souvent oblongues, simplement dentées, nues en dessus, colonnenses en dessous. Fleurs grandes, roses et solitaires. Pédoncules et calice sans aiguillons. Tube arrondi. Sépales ovales, pointues et entières.

Quoique cette espèce soit cullivée depuis long-temps, je n'en ai pas encore ohservé dans les jardins. Les spécimens qui in'ont servi pour cette description se trouvent daus l'Herbier de M. Banks.

Il paraît, par les documens que l'on troure dans cette précieuse collection, qu'Aiton, Inrsqu'il publia sa première cidition de l'Horlus kevensis, consulta les manuserits du docteur Solander, qui rapportent sous la mêne dénominalion deux plantes diffirentes, l'une qui est le R. froxinifolia, ut lit seconde, celle que nous venous de décrire, et qui n'y comple que comme rariété; mais on a pris pour phrase caractéristique celle d: notre blanda. Et d'ailleurs la figure donnéc par Jaequin, auteur aussi exact que savant, ne laisse alicun doute à cet igard. 
Fota. M. Sabine cultive le R. blanda; mais M. Lindley ne le connaissait pas encore vivaut, lorsqu'il a publie su monographic. Je n'ai jamais va les aiguillons rouges que sir Joseph Sinith dit avoir observés. Il est probable qu'il a fait sa description d'après la figure dessinèe par marlemoiselle Lawrence, yui ressemble au $R$. nitida. II. Nunziès a trouvé le $R$. blanda sur les côtes montagueuses nord-ouest de l'Amérique septentrionale.

\section{LSPECE. - ROSA FRAXINIFOLIA.}

R. Flatior, inermis. Ramis strictis, glaucescentibus ; foliolis opacis, undulatis, impubibus. Linde. Mon. p. 26 .

R. Virginiana. Mul. Dict. no 10.

R. Fraxinifolia. Lonk. Halz. 301. - Gmen. Bad.-1ls. 2. 413. - Kin. in Bot. reg. t. 468.

R. Blanda (a). Solanden. MS.-J J Je. Fragm. ;o. t. 105.

R. Corymbosa. Bose. Dict. d'Agr.-Desf. Cat. hort. par.

R. Rilpina.

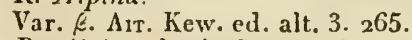

R. Alpina, lavis. Piedout. Hos. t. 5 7. t. 19-Lawr. hos. t. 78 .

Se trouve a Terre-Neuve. Herb. Banks. ( $V$. $\nabla$. cult. à Paris et euvirons.)

Au premier coup d'œil, ce rosier ressemble au li. cinnamomea. Rameaux droits sans aiguillons, d'un pourpre foncé, couverts ou,sil'on peut le dire, firdés d'une poussière bleue pâle. liejetons portant à leur base un petit nombre d'aiguillons sétifurmes. Feuilles opaques sans pubescence. Stipules longues; furt dentées vers leur extrémilé qui est aplatie. Pétioles inerimes. Folioles 5-7, lancéolées, simplement dentées, d'un vert grisâtre en dessus, glauques en dessous. Fleurs petites, rouges, portées par quelques cimes florilères. Bractées elliptiques, nues, frangées sans dentelıre. Pédoncùles plus courts que les feuilles. Tube du calice globuleux, comprimé et souvent coloré. Sépales ovales, entières avec une longue pointe intérieurement hispide. Petales presqu'en cœeur, "un peu convergens. Disque peu apparent. Style velu. Fruit petit, rond ou oblong, et d'un rouge obscur.

Entre deux dénominations, j'ai cru devoir choisir la plus ancienne, et ne pas confondre cette plante avec le $R$. blanda. Quant all nom de Miller, personne ne peut raisonnablement le sontenir. La description du $R$. corymbosa de Bosc s'ac- 
corde si parfaitement avec cette espèce, que je ne doute pas qu'elle ne soit li inême; et il était si peu vraisemblable que celle-ci fat une variété du l. blanda, que, dans la dernière édition le l'Hortus keverisis, elle a été considéréc comme ne différant point du $R$. alpina.

Elle a été recueillie à Terre-Neuve par S. Jos. Banks. Le défaut d'aiguillons distingue cette plante de la plupart de celles de la tribu. Le $R$. blanda, lorsıu'il est privé d'aiguillons, ce qui arrive souvent, se reconnaît d'abord à la villosité de ses ramcaux. Le $R$. cinnamomea, dans un état semblable, peut se reconnaître à ce même caractère, en y ajoutant ceux de la grandeur des leuilles, et la longueur des sépales qui sont contournées en leurs bords.

"Depuis la publication de la Monographie de M. Lindley, le $R$. blanda se trouve dans le jardin de $M$. Sabine; il est maintenant facile de le comparer arec ses voisins en pleine vígétation. "

\section{7․ ESPECE. - ROSA CINNAMOMEA (LINDL., Mon., p. 28.)}

R. Elatior cinerea. Ramis strictis; aculeis stipularibus, rectiusculis ; stipulis dilatatis, undulatis; foliolis oblongis, rugosis, subtùs tomentosis.

Var.. . Fonırs ovalibus, obtusis.

R. Minor, etc. BauH. Hist. 2. 38.

R. Cinnamumea. Bess. Eyst. vern. ord. 6. fol. 5 .

R. Cinnamomea. Lins. Sp. 7o3. - Willd. Sp. 2: ro65. - Alz. Ped. 2. 138. - Moenscr. Meth. 687. - Lawr. Ros. t. 34. - Bub. Tauro-Cauc. 1. 393. - Gmez. Bad.-Als. 2. 4m. - Schranck. Mon. c. ic. - Pohl. Bohem. 2. $170 .-\Lambda$ r. Kew. ed. alt. 3. 259.-Pers. Syn. 1. 47.-Eng. Bot. t. 2. 388. - S Siтн. in Rees in loc. - Rav. Enum. 52. a et b. Woods. in Act. Lin. 12. 175.-liedodt. Ros. 1. 105. t. 37. 133. t. 51.Pronv. Nom. p. 19. - Idem. Solnm. p. 19.

R. Fecundissina. Munch. Kausv. s. 279. - Doro1. Harb. 2. 3;3. HoFrm. Deutsch. A. 175. - BRAT. Lus. 1. 339. - Fr. dan. t. 1214. Rотн. Germ. 3. 557 .

R. Majalis. HerM. Diss. 8. - Desf. Atl.?

R. Var. R. Fuvvialis. Foliolis (ovatis) acutis.

R. Fluvialis. Fu. dan. 868. - Retz. Scand. 120. - Pers. Syn. 1. 47. R. Arvensis. Linv, fide $\Lambda$ fzelii.

Se trouve en Danemarck ( $F_{L}$. dan.); en Belgique (Hofrmav); en Portugal (Brotero); en Allemagne (Roth) ; en Suisse (Schleigheir, llooxer); en France (Decandolle); en Bohème (Pohl); an Caucase (Bob).

Var. $\beta$ en Danemarck (Fu. dan.); en Suisse (Hooken). ( $V$. v. c. et s. sp. Lind.. V. fig. N.) 
Ariuste gris, de 5 a 6 pieds. Rameaux droits dans l'individ: simple, faibles et étalés dans la variété double. Aiguillons forts, d'un brun pâle, presque droits, placés par naire sous les stipules. Rejelons plus couverts de soies et d'aignillons. Feuilles approchies. Stipules larges, rugueuses, concaves, rougres a leurs bords et au centre, quelquefois frangées et velues. Pétioles faibles et sans aiguillons. Fotiotes 5, rarement 7 . lancéolées, simplement deutées, rugucuses; opaques, slabres, d'un vert gris en dessus, cotonneuses et bleıâtres un dessous, concares dans les simples, et plates dans les douibles. Fleurs solitaires, ou 2 à 3 , d'un rouge pâle, petites daus la variété double comme dans les simples. Bractées larges, cotonneuses, rugueuses, concaves, bleuâtres, teintes de rouge à leur base et en leurs bords. Pédoncules nus. Tube du calice et sépales sans aiguillons; celles-ci, très-étroites, et plus longues que les pétioles, s'étalent sur la fleur, sont convergentes sur le fruit, et cotonneuses en leurs bords. Pétales concaves, presqu'en cour. Disque peu appalrent. Siyles trisvelus, distincts. Fruit rond, nu, cranoisi, couvert d'une joussière comme de cire, etcouronné sur la convergence des sépales.

Cette espèce, dont on a trouvé un individu dans le bois d'Aketon pris de Pontefract, a passé pour indigène d'Angleterre, ce que je ne crois pas fondé en raison. Elle est commune dans une grande partic de l'Europe, croît dans les buissons et fleurit de bonne heure au printemps. Mais elle so trouve en plus grande abondance dans les climats tempércis au sud que vers ceux dı norl, oủ se trouve plus particulit:rement le Majalis. Celui que nous nommons ainsi, et dont la variéti double s'appelle $R$. $d u$ St.-Sacrement, n'est qu'unc variété du cimnamomea. Celui de Desfontaines, recucilli au nord de l'Afrique, doit encore s'y rapporter. Linné a ccrtainement confondu les deux espèces, comme on le voit par son herbier (1), oủ ils sont placés sous le même nom. Cependant, la plante qu'il avait sous les yeux quand il composa sa seconde edition du Species ptantarum, est sans contredit le cinnamomea du jardin d'Upsal. L'autre a été ajoutée depuis, et elle peut aroir été décrite d'après quelque plante de la Sucde. Le doctenr Afzelius, dans son premier essai, avance que le $R$. spinosissima de Linné est un cinnamomea, sans

(1) S. Jos. Smith en est lo posscsseur, l'ayant achclé avec ses manuscrits.

(Note de M. Joffrin.) 
expliquer pourequi. Mais cela re peut pais ètre car daus la 2:. idition du Flora suecica, il est dit que le fruit est noir, ce qui est particulier au $R$. spinosissima, et jamais à aucun individu de cette tribu.

La variété double est bien plus commune que l'espèce simplè, et présente même des différences notables; les rejetons ne sont ni aussi forts ni aussi droits; les feuilles sont aplaties et unn concaves, ce qui paraît venir de quelque altúration dans lc type, cumme nous voyons qu'il arrive ì d'autres espèces transportées de lcur lieu natal et cultivées.

M. Woods observe avec raison que le cinnamomea de Roth, est le lutea bicolor.

Je possède quelques échantillons du fuviatis de la Flora danica, recueillis en Suisse par $\mathbf{1 1}$. Hooker. La plante paraît plus petile, les feuilles plıs courtes, et les aiguillons plus rares que dans l'espece. Les fleurs sont aussi d'un rouge plus foncé. Plısieurs en forment ume espèce distincte; mais il est probable qu'il tient plus du cinnamomea que dumajalis, entre lesquels il peut être placé comme intermédiaire. Ic cinnamomea d'Hermann est un spinosissima.

M. Lindley place à la suite du cinnamomea les trois espéces suivantes, que je n'ai pas osé adinettre sur une simple description.

\section{ROSA TAURICA.}

P. Elatior cinerea. $\Lambda$ culeis sparsis, dehilibus ; ramis strictis, apicem versus inermibus; foliolis oblongis, rugosis, subtìs villasis; sepalis contrapositis; stylis perrectis, glabriusculis. Buв. Taur.-Cauc. 1. 394.Poir. Enc. bot. suppl.

\section{ROSA DAVUICA.}

R. Elatior ramosissima. Ramis tenuibus, coloratis; aculeis stipularibus, patentissimis, subrecurvis ; stipulis linearibus ; foliolis oblongis, rugosis, subtìs tomentosis, alté serratis. PALLAs. Russ. 61.

Se tronve en Daourie. Fl. en juin.

coveses

\section{ROSA ARISTATA.}

R. Foliis superioribns, subbijugis; petiolo in spina producto. Prcot LAPEXR. Fl. pyr. t. 105. Hist. 285.

Se trouye prés de Barèges. 


\section{ESPËCE.-ROSA MAJALIS. (LiNDr., Mon., p. 34.)}

R. Humilior, cæsia. Ramis strictis, coloratis; aculeis sparsis, subæqualibus; stipulis linearibus; foliolis oblongis, planis, subtus gla cis, tomentosis.

R. Majalis. Retz. Obs. bot. 3. 33. - WanL. Lapp. I 41.

R. Mutica. Fl. dan. ᄂ. 688 .

R. Spinosissima. Gorter. Ingr. 78.

R. Collincola. Err. Betr. 2 . Fo.

Var. f. Canescens, foliis albido-cæsiis.

Se trouve en Suède. Afz. (Swartz); en Laponie (WALt); en Danesnarck (Fl. dan.).

Var. $\beta$ en Suède. $\Lambda \mathrm{fz}$. (Swartz). ( $V$. v. c. et s. sp. )

Ce rosier a 3 ou 4 pieds d'élévation : il est très-glauque. Rameaux droits, en verge. Aiguillons faibles et droits, épars ou placés sous les stipules. Riejetons garnis, comme les autres espèces de la tribu, d'un grand nombre d'aiguillons presque égaux, et de soies. Stipules ètroites, marginales, a vec une expansion quelquefois arrondie. Pétioles un peu cotonneux et glandulaires, avec quelques aiguillons faibles, petits et droits. Folioles ordinairemen: 7 , ovales ou obovales, obtuses, simplement dentées, aplaties, très-glabres, glauques des 2. côtés, surtout en dessous où elles sont un peu cotonneuses. Fleurs solitaires, petites, en forme de coupe, d'un rouge pâle. Bractées solitaires, ovales, concaves, nues et pointues. Pédoncules et Calice cotonneux. Tube rond et petit. Sépales presque simples, pointues, plus longues que les Pétales. Ceux-ci émarginés. Disque peu apparent. Styles séparés. Fruit rouge-orangé, sphérique, nu, couronné par les sépales.

Cette espèce est confinée en Suède et en Danemarck, ou elle paraît croître abondaumment. Ses caractères sont apparens et son port diffère beaucoup du $R$. cinnamomea, a vec lequel on l'a confondue. D'après des échantillons qui ont été envoyés à M. Hooker, il paraît que Srrartz avait le dessein de la partager en 3 espèces qu'il appelait cinnamomea, cinerea et turbinella. Leur aspect a beancoup de similitude et je n'ai pus y décou vrir de différence remarquable.

Il n'existe d'autres figures de cette jolie petite rose que celle de la Flore danoise. 
- Je soupconne qu'un rosicr nommé carolina ct qui se troure au polager de Versilles, est un majalis ou sa varieté. Je le cultive depuis qu'il m'a été envoyé par M. Sabine. Il a fleuri en 1823 .

19. ESPECE. - ROSA MACROPIIYLLA. (LINDE., Mon. 1. 53 , avec fir.)

R. Inermis. Foliis Iongissimis, parcè glandulosis; foliolisque İaceolatis, sublùs lanatis; sepalis angustissimis; petalis apiculatis, longioribus.

be trouve dans le Gossan-Than. Waluich. ( $V$. s. sp. in Ilerb. PAXKS.

Rameaux sans aiguillons, d'un brun rougeâtre. Stipules concares, dilatees, arruées, aiguës, colorées, nues. Pétioles quelquelois de 9 pouces de longueur, garnis d'un coton épais, sans aiguillons, arec quelques glandes dans le duvet. Folioles de j̇ 11 , lancéolées, aplaties, veinées, simplement dentées, les dents très-pointues, toutes d'un vert foncé nuancé de pourpre, nues a leur surface et d'un blanc cotonneux en dessous. Bractées annelées el colorées de rouge, lancéolées, très-longues et larges à leur base, presque entières, nues excepté la nervure du milieu qui est velue des 2 en̂tés. Pédoncules velus, avec quelques soies colorér's inégales. Tube du calice oblong et nı. Sépales très-longues, étroites et triangulaires, simples, dilatées et denteléë à leur extrémité dont le revers offre quelques points colorés. Pétales obovales, plus courts que les sépales, avec une petite pointe coloréc de rouge. Anchères oblongues, larges. Disque très-grand, un peu élevé a l'orifice. Ovaires 28 , très-velus. Styles velus, saillans et séparés.

Cette plante est une des plus nouvelles acquisitions envoyées du Gossan-Than à S. Jos. Banks par le D. Wallich.

Il differe de l'alpina dans la forme de ses stipules et de ses grandes bractées, et de plus les feuilles sont très-cotonneuses, ce sont les plus grandes que j'aie encore rues. Il ne peut être confondu avec aucun autre, et doit être considéré comme le chaînon qui lie cette tribu arcc la suivante 


\section{Ve. TRIBU. - PIMPINELLIFOLIZE.}

Caractères particuliers.-Setigeræ; armisconfertis, subconformibus; v. inermes; ebracteatæ (rarissimè bracteatæ). Foliola ovata, v. oblonga. Sepala connirentia, persistentia. Discus subnullus.

Cette tribu diffère naturellement de la dernière par son port, ou sa physionomie habituelle, quoique dans les caractères artificiels, il faut l'avouer, elles approchent beaucoup l'une de l'autre. L'on peut toutcfois les distinguer par un plus grand nombre de folioles, qui varie de 5 à 13 et même à 15 , au lieu que les premières ne varient que de 5 ì 7 . Ces folioles sont ordinairement ovales, rarement oblongues, jamais lancéolées. Les fleurs sont presque toujours privées de bractées, excepté dans les $R$. alpina et Sabini. Ceux-ci, ayant les sépales conniventes el permanentes, ne peuvent être confondues avec les familles précédentes, ni avec celle qui va suivre, à cause de l'exiguitté de leur disque.

Le $R . W$. Wodsii diffère de ses co-espèces par la forme de ses feuilles; la variété du cinnamomea n'offre pas un aussi grand contraste.: mais l'un et l'autre ont des aiguillons stipulaires, et dont il n'y a pas d'exemple dans la présente tribu. Observons encore que tous lés péricarpes ont une surface différente.

\section{ESPE்CE. - $R O S A$ ALPINA.}

R. Inermis. Fructu elongato, pendulo; peduuculo lispido. Lisra. Non. p. $3 \%$.

R. Rubra, preeox flore simplici. Besi. Eyst. vern. ord. 6. fol. 5.

R. A Lpina. Linn. Sp. jo3. - JacQ. Austr. 3. 43. 2. 139. - WILLD.Sp. 2. 1075. - LAWR. Ros. t. 3o.-DeCaid. FI. fi. 4 . 4 6.536. - Pers. Syn. 2. 49. - Ait. Kew. ed. alt. 3. 265. - Smitn. in hees in 1. - Lindere. in Bot. reg. t. 424. - Prorv. Nom. 52. - idem. Somm. ז $\mathrm{g}$.

R. Rupestris. Chantz. Anstr. 85.

R. Monspeliaca. Govan. Monsp. 255.

R. No. 8107. HALL. Helv. $24 \mathrm{r}$.

R. Inermis. Mlle. Dict. no. 6.- Tun R Diar, act. 128.

R. Hybrida. Vitz. Delph. 3.554 .

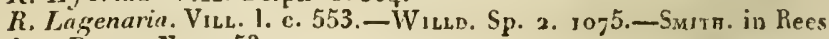
in l. - Pronv. Nom. 53.

K. Biflora. Krocr. Siles. 2. 157 . 
Var. R. Prrenaica: Calycis tubo pedunculoque hispidulis.

R. Prrenaïca. Gouan. Illustr. 31. t. 19. - Willd. Sp. 2. 10-6, DeCAxD. Fl. fr. 4. 446 . - Pers. Syn. 2. 49. - Sujth. in Rees in 1. - La-

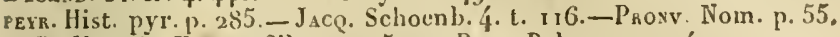

R. Hispirla. Krofк. Siles. 2. 152.- Pонц. Rohem. 2. 174.

R. Turbinata. Willd. Delph. 3. 55o?

R. Alpina d. Decand. Fl. fr. 6. 536.

Var. $\gamma$. Pendulina. Foliolis pluribus caulcque coloratis.

R. Pendulina. Lins. Herb. - Ait. Kew. ed. 1. - Wilıd. Sp. 3. so-6. - Pers. Syn. 2. 49. - Lawr. lios. t.91. - Mit. liew. ed.alt. 3. 265.- Sи1тн, in Rees in 1 .

R. Alpina pendulina. Redovt. Ros. s. 5 7. t. 17.-Prosv. Mon. p. 55.

Var. d. Pimpinellifolia. Omnibus partibus minor.

R. Pincpinellifolia. IVıL. Delph. 3. 53\%.

R. Glandulosa. Belland. in Act. taur. I79o. p. 230.

R. Pygmau. Вев. Taur.-Cauc. I. 397 .

R. Pyrenaïca. Var. B. Smiru, in Rees in 1.

Se trouve dans les $\Lambda$ lpes de l'Autriche (JAcQ.); montagnes de la France méridionale (Houenv., Decand.); en Silésie (Krooker); en Bohéme (PoHL); cn Dauphiné (VILlaRs); mais surtont en abondance en Suisse (HoOKER), où il croît à 6600 pieds d'élévation. ( $V$.v. et s. sp.)

Arbuste de 7 à 8 pieds de haut. Rameaux presque droits, d'un brun verdâtre, ordinairement d'une tcinte glauque; sans aiguillons ou rarement garnis de quelques aiguillons faibles i) la base des rejets. Stipules étroites, dilatées à leur extrémité, nues, frangées de glandes. Pétioles garnis de poils ou de glandes pédicellées. Folioles de 5 à. 9 , minces, ovales, aiguës aux deux extrémités, doublement et grossièrement dentelées, nues, mais la nervure du milieu quelquefois scabre, avec quelques courts aiguillons. Fleurs droites, rouges et solitaires. Pédoncules hispides, sans aiguillons. Tube du calice glabre ou hispide, ovale, très-allongè. Sćpales droites, étroites, simples, avec une légère pointe dilatée il leur extrémití, qui s'élìve au-dessus des pétales; les sépales sont velues au dehors; mais si le tube du calice est nu ou scabre, elles le sont aussi. Pétales obcordés, concaves. Disque large et comprimé. Styles velus, séparís. Fruit pendant, rougre-orangé, oblong, en forme d'amphore, et couronné par les sépales, qui sont convergentes.

Je crois que la plupart des auteurs s'accordent sur presque toutes les synonymies de cette plante. Sa grande abondance dans les pays où elle croît, et les différentes situations dans lesquelles on l'a trouvée, ont induit plus d'un botaniste en erreur, suivant les apparences diverses qu'elle présente, et les a 
déterminés ì en former plusieurs espèces. Ainsile $R$. hybricle de Villars eat été noınmé d'après ses pétales entiers. $R$. lagenaria repose sur l'autorité d'une seule plante trouvée dalus les environs d'Einbrun, dans les bois de Boseadon, avie un fruit plus long et élranglé supérieurement ; et dans le biflora de Krooker, on ne saurait tronver un firuit qui puisse caractériser une espèce. S. James Sinith sépare avec raison lat variété $\beta$ de l'Horıus kezvensis de celui-ci; mais il n'est pas aussi exact en lui donnant la place qu'il lui assigne.

Ma variété $\beta$ pent se reconnaître ì son fruit hispide (1). M. Decandolle, qui l'a considérée cunnme espèce dans la Flore francaise, s'est rétracté dans son supplément, où cette variété est notíe comme $R$. alpina, $\mathrm{n}^{\circ}$. 5. La figure de Jacquin est excellente.

On a voulu aussi faire une espèce du pendulina de Linné: c'est ma variété \%. La plante se trouve dans nos jarding comıne dans son herbier. Ses fenilles et ses tiges colorées, sa disposition à prendre plus de folioles que la commune, arec des fleurs plus foncées, ne sont pas des caractères suffisans pour en constituer une espèce. Jo ne sais non plus comment son fruit, étant pendant, écarlate, glabre, luisant, très-allongé, courbé et arqué, le distinguerait du premier alpina, comme on le remarque dans l'Encyclopédie de Rees.

D’après les caractères de Villars et de Bellardi, on a cru généralement que le pimpinella du premier, et le glandulosa du second se distinguent à peine de l'alpina. S. Jauses Sinith l'a déjà remarqué, et je me range rolontiers à ses opinions, en croyant qu'il convient de les réunir à l'espèce, comme le pyrenaïca de Gouan. Decandolle dans son voluıne supplémentaire de la Flore française les a notablement séparés de l'alpina, et je ne sais sur quel fondement il leur a appliqué une description qui paraît convenir tout-à-fait au rubiginosa. Le $p y g$ maea du savant auteur de la Flore tauri-caucasienne, auquel l'alpina de quelques index de Pallas est cité, paraît être le mêne que celui que ce grand voyageur appelle des montagnes, ou une variété du rubella, ì raison de summi ranuli floriferihispiduti. Quoi qu'il en soit, je ne saurais douter que l'aipine de Pallis, Elora Rossica, n'appartienne à l'espèce suivante.

(r) J'en possède un échantillon recueilli dans la vallée d'Aoste. Le fruit ressemble en grand a celui du phascena ampullaceum. 
" La variété $\delta$ dont il est question se trouve dans nos jardins; Inais la culture en a augmenté les dimensions infiniment petites à l'égard des autres variétés. Le fruit, qui est rond, est évidemment altéré par cette même culture, et peut-être le croisement avec une espèce voisine.s

\section{ESPÈCE. - ROSA RUBELLA.}

R. Armis confertis, aqualibus; fructu elongato, pendulo. Linoz. Mon. p. 40. - Pronv. Somm. p. 18 .

K. Pendula. Roth. Germ. 2. 5or.

R. Alpina. Palz. Inss. 6I.

R. Rubella. Eng. Bot. t. 2521. - Smitr. in Rees in 1. - Woods. in Act. lina. 12. 177.

R. Polyplyylla. Wiılo. Enum. suppl. 37.

R. Candolleana. Red. hos. 2. p. 45 . cum fig.

Var. $\beta$. Melanocarpa. Fructu nigro, fusco, breviore.

Se trouve en Angleterre (Smitu, Wincin); en Allemagne (Rотн); commune en Sibéric depuis la chaîne des monts Ourals jusqu'en Daourie (PALL.); dans les plaines d'lset, à Obo, Irtesch et Jenisch. idem. ( $V$. v. cuit. et sp.)

Tige de 3 à 4 p. Rameaux droits, rougeâtres, entièrement couverts jusqu'aux extrémités de soies et d'aiguilions faibles. Stipules élargies vers leur extrémité, échancrées à leur limbe, frangées de glandes, nues. Pétioles peu glandulaires et sans poils comme les folioles, qui sont de 7 à 11 , presque planes, ovales, pointues, simplement et quelquefois doublement dentées, d'un vert foncé en dessus, plus pâle en dessous. Fleurs solitaires sans bractées, pâles ou d'un roure foncé. Pédoncules plus hispides que le tube du calice. Sćpales droites, nues et plus courtes que les pétales qui sont concaves et émarginés. Disque trìs-mince. Fruit pendant, long, ovale, écarlate, couronné par des sépales courtes et convergentes.

Cette plante est probablement une de celles que Linné a confondues sous le nom de pendulina. Il n'y a pas de doute sur la synonymie de Roth, attendu que ses caractères se rapportent a toute autre rose européenne que le pendulinc. Je suis également persuadé que Pallas avait cette plante en vue en déterminant son alpina. Il dit qu'elle varie selon le lieu oủ elle croît. Il l'a observée avec des tiges et des pétioles armés d'aiguillons capillaires, minces, nombreux, ce qui conviendrait assez bien au rubella, mais aussi à l'alpina. Aussi, 
d'après mes conjectures, il en avait été fait mention longtemps avant qu'elle parat en Angleterre et arant qu'on lu publiât danz la Botanique anglaise, où on la donne comme nouvelle, arec une description remplie d'erreurs. Ce qui est dit dans l'Encyclopédic de hees au sujet du calice est également applicable au spinosissima, et je crois que l'observation de 11. Backhouse, que les feuilles se fermaient la nuit, est aussi une méprise, car je n'ai jama is pu découvrir un pareil phénomène, malgré les soins que j’ai pris à cet égard.

M. Woods est le premier qui ait remarqué que les tiges et les rameaux couverts de soies entremêlé es de quelques aiguillons le distinguent suffisamment du spinosissima. Je dois y ajouter le fruit long, rouge et penché qu'il n'a point vu. Il est plus difficile de le distinguer du stricta; mais leurs caractères principaux et différentiels seront indiqués à l'espèce suivante.

Le $R$. polyphylla de l'Énumération des plantes du jardin botanique de Berlin par Willdenow, que M. Ker a bien voulı me communiquer, ne paraît différer en rien de celui-ci, et le suavis du même ou vrage scmble se rapporter ì mon stricta. La variété $\beta$ est parfaitement intermédiaire du rubella et du spinosissima. Je l'ai obtenue de la pépinière de Ml. Lee sous le nom de rubella.

a Le rosier cultivé chez M. Noiselte se rapproche beaucoup plus de l'alpina de Pallas que du rubella; la culture et le changement de localité ont da lui faire subir de grandes altérations.

\section{atimencosectsetave}

\section{$22^{\circ}$. ESPE்CE. - ROSA STRICTA.}

R. Ramosissima. Piamulis inermibus; fructu elongato, pendulo. Linde. Mon. p. 42. c. fig.

R. Sanguisorba. Majori folio; fructu longo, pendulo. Ex Novâ- $\Lambda$ gliâ. DiLl. EIth. 315. t. 245. f. 317 .

R. Virginiana. Herm. Diss. 19?

R. Pendulina. Lins. Sp. 705.

H. Siricta. Noul. Cat. 50 .

R. Carolina. Aıt. Kew. ed. alt. 3. 260 .

f. Carolina. Lawn. Ros. t. 36. (Pessima).

R. Suavis. WıLt. Enum. suppl:' 37 .

R. Stricla. Provv. Somm. p. I9.

Se trouve dans l'Amérique septentrionale, Nouvelle-Angleterre (DıLesius); Pensylvanie (MuнL). ( $V$. v. cult.) 
Rameaux dioits, de 5 i 4 pieds, d'un vert pâle, couverts entierement de soies petites, faibles et presque égales, excepté less rxtrémités qui sont divisées en ramilles nombreuses, liahles et unes. Folioles de 9 ì 11 , arrondies, d'unc texture ferme, glauques, la paire inférieure plus petite que ley autres. Fleurs d'un rouge clair. Fruit nu avant sa maturité et couvert de quelques petites taches pâles. D'ailleur's les caractires ilu rubella.

Malegré li ressemblance frappante entre celui-ci et le rubella, je ne vois pas de difficulté de l'en séparer. Le rubella a ses rameaux faibles et penchés, ses rejetons courbés et hispiides a leur sommet; ses fenilles sont vertes, le fruit petit, les ovaires de 15 à 18 , le péricarpe ovale et un peu pointu à l'extrómité. Le stricta au contraire a les rameaux droits, les. rejetons et les ramilles (petites branclıes) sans aucune hispidité; les feuilles sont quclquefois glauqnes, le fruit long et couvert, avant si maturité, de petites taches pâles. Les ovaires de 25 a 35 ; les péricarpes sont ronds, gros et très-velus. Souvent le rubella est armé d'airuillons, et jamais le stricta. On m'objectera peut-être qu'en d'autres occasions j'ai rejeté de meilleurs caractires comme insuffisans pour distinguer des espèces avec unc apparence de raison. Mais quand on se rappelle qu'il n'y a pas un scul exemple qu'il se soit trouvé en Europe ancun rosier de l'Amérique septentrionale, et qu'il faut que celle-ci fasse exeeption si on ne la sípare point. du rubella, j'aurai pour moi l'importance de la distribution géographique.

Elle est counue en Augleterre depuis Dillen, qui la troura lans le jardin de Shérand, oú elle était venue de graines envoyées de la Nonvelle-Angleterre, et anssi par la figure qu'il a donnée dans son nombreux Hortus eltamensis. Fiute d'it voir donné l'attention nécessaire à sa description, il en est résulté beaucoup de confusion pourle stricta, car la figure a tonjours été citée depuis pour une variété d'uncespèce diffírente, probablement par l'effet de la culture; en sorte que mon alpina, var. \%, a été jugé une plante d'Amérique septentrionale. Grand einbarras pour les botanistes le ce pays qui l'ont cherchée long-temps sans aucun succès. Pour en déconrrir la cause il est nécessaire de remonter à l'origine de ce rosier.

Le specimen du'pendulina de l'H crbier de Linné appartient, romme je l'ai dej.ji olssepré, a la plante que nous cultirons sous ce nom dans nos jardins. L'extrémité des petites branches 
est assez semblable à la figure de Dillenius; il a'indique pas d'où elle lui est venue, et elle ne lui a peut-être été connue que dans l'état de siccité, ce qui explique suffisamment son erreur lorsqu'il cite l'Hortus elth., dans son Species plantarum, ${ }^{\text {ere }}$. édition. La phrase spécifique du penclulina est fructibus oblongis pendulis, qui sert à le distinguer le ses coespèces, parce qu'alors il ne connaissait pas l'alpina. Mais avant que la $2^{c}$. édition ne parat, il put se procurer cette dernière plante, et alors il jugea nécessaire de changer le caractere du pendulina par ces mots: Germinibus ovatis, glabris; pedunculis cauleque hispidis; petiolis inermibus; fructibus pendulis. Ce qui prouve complètement qu'il s'attache au Stipites innumeris spinis, tenuibus et innoxiis, deorsùm flexis, geminis de Dillenius, qui ne convient pas au pendutina d'Aïton, pour en faire une espèce particulière. Dans Ja $1^{\text {ere }}$, édition de l'Hortus kezwensis, sa définition se convertit en inermis;germinibus oblongis; pedunculis petiolisque Iispidis; caule ramisque glabris; fructibus pendulis, qui se rapporte évidemment au pendulina de nos jardins. Quelle fut la raison de ce changement? On ne la donne pas, car le Dr. Solander, dont les manuscrits ont servi à la classification du genre, savait très-bien que ce n'était pas la plante de Linné. Voilit pourtant l'origine de l'erreur, qui ne saurait diminuer l'estime que l'on doit à cet excellent ouvrage, et qui aurait da être connue plus tôt.

"Le rosier que je tiens de $M$. Sabine se rapporte en tout à la description de $\mathrm{M}$. Lindley; il a fleuri en 1825 . "

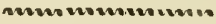 \\ 23․ ESPĖCE. - ROSA ACICULARIS.}

R. Elatior; aculeis acicularibus, inæqualibus; folinlis glaucis, rugosis, convexiusculis; fructu obampullaceo, cernuo. Lisol. Mon. p. 44 . c. fig.

R. Acicularis. Pronv. Somm. p. I9.

Se trouve en Sibérie (Bell). Pallas. ( $V$. v. cult. et s. sp. Herb. Cel. LAMBERT. )

Buisson épais, d'environ 8 pieds. Branchcs droites, les plus jeunes glanques, les adultes brunâtres, arnées de très-faibles aiguiltons droits et de quelques soies. Feuilles denses, opaques, trìs-glauques. Stipules étroites, nnes, frangées de glandes, un peu élargies a l'extrémité. Pétioles vert-pâle, nus 
ou un peu velus, faibles, avec des attaches très-longues. Folioles environ 7, ovales, convexes, divergentes, presque nues, bleuâtres en dessous. Fleurs solitaires, d'un ronge pâle. Bracties ovales, convexes, nues ainsi que le pédoncule. Tube du calice nu, elliptique. Sépates très-étroites, quelque peu divisées, velues, 3 lois aussi longues que le tube. Pélates obovales, émarginés, étalés, plus courts que les sépales. Disque large, un peu élevé. Styles velus, séparés. Fruit obovale, étranglé vers le haut, de couleur orangée jaunâtre, nu, un peu penché, couronné des sépales conniventes et épaissies à leur base.

Addition intéressante à la tribu des pimprenelles, et que nous devons à $\mathbf{l l}$. Bell, qui l'a découvert en Sibérie. A en juger d'après des plantes communiquées par feu M. Doon a M. Sabine, il paraît être le liamtschatica de son Hortus cantabrigiensis. L'inégalité de ses aiguillons le distingue des trois espèces précédentes; il diffère aussi des autres par la plus grande proportion de ses feuilles glauques et rugueuses. Sous le: premier rapport il est surpassé par le $R$. Sabini; mais les aiguillons forts et courbés de cetle dernière plante, qui paraissent parmi les soies, et en forme de poignard quand celles-ci manquent, ne permettent pas de les confondre, sans parler de leur différence complète à d'autres égards.

"C'est le premier rosier qui se couvre de feuilles, ct àcette époque il est remarquable par la couleur jaune de ses feuilles naissantes comme si on en avait enlevé l'épiderme.

"Ce rosier, que je cultive et qui se multiplie aisément, paraît très-rustique. Il fera un bon effet dans les jarúins paysagers par ses fleurs nombreuses et latérales.”

\section{ESPECE. - ROSA SULFUREA.}

R. Stipulis linearibus, apice dilatatis, divaricatis; foliolis glaucis, planiusculis; tubo hemispliarico. Linde. Mon. p. 46.

R. Flava pleno flore. Cuus. Cur. post. 6 .

R. Lutea maxima. f. pl. Less. Eyst. vern. ord. 6. fol. 2.

R. Lutea multiplex. Paks. Parad. 417. no. 17. t. 415. f. 6, - Gen. Exac. 1267 .

R. Luleaf. $p l$. Ras. Hist. r $\left\{7^{5}\right.$. no. 3 r.

R. Hemispliarica. He в. Diss. is.

R. Glaucophylla. EnR. Deilr. 2. 69.

R. Sulpluter. Ait. Kew. 2. 201. - Wirld. Sp. 2. y065.-Lawr. Ros. t. 72 . - Pers. Syn. 1. 47 - Gnez. Bad.-Als. 2. 404. - Kra. liewist. u. 46. - Siritu. in Rees in $1 .-$ Kedout. hos. I. 29. t. 3. 
R. Lutea. Вкот. Lus. 1. 33 .

Probablement originaire d'Orient (Cuusrus.) ( $V$. v. cult.)

De 4 à 5 pieds en Angleterre, mais de 8 à $g$ en France feuillé aux extrémités de la tige. Branches vert-jaunâtre ou brun, armées de soies et d'aiguillons pâles, épars et inégaux, les plus longs un peu courbés, les autres faibles et droits. Feuilles d'un vert glauque foncé. Stipules étroites, plates, dilatées en fourche et grossièrement dentelées i leur sommet, sans pubescence comme les folioles. Pétioles un peu glandulaires, garnis de quelques aiguillons petits et droits. Fotioles 7, obovales, plates, simplement dentées, d'un vert grisâtre en dessus, bleuâtres en dessous. Fleur's très-grandes, extrêmement délicates et transparentes, jaunes, toujours doubles. Point de: bractées. Pédoncules et Calices ou nus ou glandulcux. Tube hémisphérique.

Cette plante est parmi celles du genre une des plus riches en floraison. On ne l'a jamais vue simple ni disposée à le devenir. Sa patrie est encore inconnue. Les premières notions que nous en ayons eues viennent de Clusius, quile premier la remarqua en examinant un petit jardin artificiel en papier, orné d'arbustes de différentes sortes, parmi lesquels se trouvait une rose jaune double. Ce jardin artificiel arait été apporté de Constantinople, comme il le dit; et, par le moyen de ses nombrcux corresjiondans, il se procura bientôt des individus vivans qui ont probablement donné naissance anx rosiers que nous prossédons anjourd'hui. Il faut que Linné ne l'ait point connue lorsqu'il a pensé qu'elle était une variété de son R. eglantina (lutea $W$.)

On éprouve tonjours beaucoup de difficultés pourl'épanonissement de ses belles fleurs. S. Jos. Banks dit l'avoir vu dans le plus bel état de floraison dans un sol marécageux. Le bel échantillon qui a servi de modèle pour la figure qu'Édourard Sydenham a donnée dans le Register, venait du comté d'Oxford, et il était si parfait qu'une dame qui allait au spectacle, en ayant mis un bouton dans son sein, il s'y épanouit dans le cours de la soirée.

- Je suis étonné que les botanistes, et Lindley lui-même, n'aient point parlé de la variété minor, que l'on voit si rarement fleurir. Les rameaux sont couverts de soies jusqu'i l'extrémité, ct les feuilles très-rapprochées; cette variété n'est que de collection." 


\section{ESPECE. - ROSA LUTESCENS.}

R. Armis ramorum confertissimis, inacqualibus, gracilibus, reflexis ; ramulorum minimis, subiequalibus; foliolis planis, impubibus, simpliciter serratis. LINDL. Mon. p. 47. cum fig.

R. H:spida. Curt. Mag. t. 1570 . (Mala.)

R. Lulescens. Pursch. Am. sept. vol. 2. in suppl.

Originaire, vraisemblablement, de Sibéric. $(V$. v. cult. $)$

Arbuste élevé, viģoureux, mais peu agréable à l'œil. Branches perpendiculaires, presque droites, d'un brun sale, défendues par une innombrable quantité d'aiguillons faibles, inégaux, d'un brun pâle, penchés et mêlés. Rameaux avec un pareil nombre de soies décolorées, sans pubescence. St $i$ pules très-étroites, plates. Pétioles inermes. Folioles, de 7 i 9 , ovales, plates, dentelure simple. Fleurs moyennes, jaune-pâle, solitaires. Point de bractíes. Pédoncules et Calice nus. Tube orale, beaucoup plus court que les sépales qui sont entieres. Disque aplati. Ovaires, environ 5o. Styles velus, distincts. Fruit gros, ovale, noir, porté sur une queue charnue, et couronné par des sépales courtes et conniventes. Péricarpe volumineux, cramoisi et rugueux.

Pursh a été induit en erreur en comprenant cette plante parmi celles de l'Amérique septentrionale, parce qu'elle était connue dans les pépinières sous le nom de rose jaune d'Amérique, ce qui n'est foudé sur aucune autorité. Je partage plutôt l'opinion du savant éditeur du Magasin botanique, en la considérant comme native de la Sibérie, á cause de sa ressemblance avec les plantes de ce pays, ressemblance qui ne peut s'appliquer à celles dı nord de l'Aınérique. Il paraît qu'elle a été cultivée à Chelsea, piar M. Fairbairn, et c'est probablement de cette souche que sont venmes toutes les plantes que nous cultirons dans nos jardins. Peut-être une plante de si peu d'importance ne mérite pas un nom plus harmonieux qu'il n'est ancien, et je réclame à ce sujet l'indulgence des anıateurs.

Ce rosier se distingue parfaitement du spinosissima dans sa manière de croître, surtout par ses rejetons vigoureux, droits, couverts d’aignillons épars, par la couleur pourprée de ses feuilles en automne; les rameaux qui partent dcs fleurs se 
distinguent notamment des branches en ce qu'ils sont garnia de tubercules portant de faibles soies, et que l'on pourrait considérer comme des rudimens d'aiguillons non formés; mais cette particularité est constante.

«Le $K$. lutescens a fletri dans mon jardin en $182 \bar{j}$. J'ai vérifí tous les caractères que lui assigne M. Lindley."

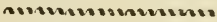 \\ $26^{\mathrm{e}}$. ESPE்CE. - ROSA VIMINEA.}

K. Ramis vimineis; armis setaceis, confertissimis, rectis, patentibus, inzerualibus; foliolis membr anaceis, planis, impubibus, simpliciter serratis. LIXDL. Non. p. 49.

li Viminea. Phosv. Somm. p. 20.

Patrie inconnue. Pallas. ( $V$. s. Herb. Lambert. (Lindl.)

Branches allongèes, faibles, délićes, qui ne ressemblent nullement i celles du spinosissima, totalement couvertes d'aiguillons sétacés, étalés, droits et inégaux, et de quelques soies. Feuilles très-longues. Folioles, de 5 à 7 , oblongues, dentelure simple, d'une teinture membraneuse. Stipules dilatées an sommet. Pétioles pédonculís. Calices nus. Tube ovale. Fleurs très grandes.

Je suis redevable de la connaissance de cette plante à M. le ch. Lambert, qui l'a trouvée dans le riche herbier de Pallas, dont il a fait l'acquisition. Son lien natal est inconnu. A en jugar par l'étiquette qui y est jointe, il est vraisemblable qu'il a été tiré de quelque jardin botanique. On ne pourrait le confondre qu'arec le spinosissima ou le tutescens, dont les rejetons faibles et minces, garnis d'aiguillons sétiformes très-épais, le distingment assez. Je ne connais aucun autre rosier ayant la même habitude. Si on devait en attribuer la cause à ce qu'il a végété dans un sol très-ounbragé et resserré, les rejetons ne seraient pas aussi couverts d'aiguillons, et les feuilles eussent été d'une texture plus mince. Son feuillage membranenx, toujours ferme et rigide, empêche qu'on ne le confonde avec aucune variété du spinosissima. Les pousses luxuriantes de ce dernier sont ordinairement couvertes d'aiguillons forts et courbés en fer de faux. Les pousses faibles n'en ont point.

"J'ai balancé si je ferais entrer cette espéce dans la monographie; mais elle peut se retrouver un jour, et l'exactitude de M1. lindley garantit qu'il a assez observé la plante de M. Lambert pour ne pas la confondre avec ses voisines.* 


\section{3․ ESPÈCE. - ROSA SPINOSISSIMA.}

R. Armis inacqualibus; foliolis planis, impubibus, simpliciter serra.

tis. LiNd. Non. 1'.50.

R. Dumeusis. Dodov. Stirp. hist. 187 . 1. 3.

R. Crnerrhadi species, etc. THal. Sylv. hirc. 35.

R. Campestris ollom. Cuus Hist. I. 116.

R. Pracox spinosa. FI. albo. Bess. Eyst. vern. ord. 6. fol. 5.

R. Cnmpestris, clc. Bavh. Pin. 483.

1. Pimpinellifolia. Ger. En. 1270 .

R. Pumiln spinosissima. A. J. Bavn. Hist. 2. 40. ג-Ra1. Hist. 1472 . no. 15. Syn. 455 .

Var. $\Lambda$. Pumila. Armis horizontalibus; fructu ovato.

* Pedunculo glanduloso vel setoso.

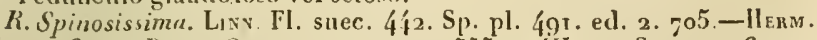
Diss. 1762 . - Котн. Germ. т. 2 17. 2.555. - WiLld. Sp. 2. 1 о67. Pers. Syn. 2. 48. - Gub. Taur.-Canc. 2. 395. - Pronv. Nom. p. 24. Itlem. Somm. [. 20.

R. Cinnamomea. Ilerni. Diss. 7.

R. no. $1106 . H_{\text {ALL }}$. Helv. 2 fo.

R. Chamarledon. Viut. Delplı, 3. 555.

17. Pimpinellifolia. V. D. - Redour. Ros. 1. 119. t. 44 .

** Pedunculo nudo.

R. Spinosissimn. Fu. Das. t. 39S. - Hoos. Angl. 21 S. - Bdzl. Par. t. 277. - All. Pedem. 2. 138. - Lawr. Ros. It. 18. 48 . - Smith. Bril. -

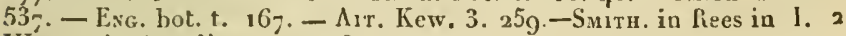
Woons. in $\Lambda$ ct. linn. 12. $17 \mathrm{~S}$.

R. Pimpinellifolia. Lisv. Syst. nat. ed. 10. ro62. Sp. pl. zo3.Moensh. Meth. GS. - Roessig. lios. t. 9 t. 25. fig. 2-Decand. FI. fr. 4 . 438. - GMel. Bad.-Als. 2. 415. - JAcQ. Fragm. 21. t. 10\%, f. 1. - ReDou'r. Ros. 1. 83. t. 29.85. t. 3o.

R. Scntica. Mill. Dict. no. 5.

R. Collina. Sснranck. Baiers, fl. no. 724. fide Rau.

Variétés du spinosissima.

R. Var. B. Reversa. Pumila; armis gracillimis, inferioribus, deflexis; fructu ovato. Lind. I. c.

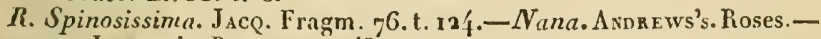
Reversa. LiNde. in Bat. reg. t. 431.

R. Var. C. Platycarpa. Pumila; fructu depresso et pedunculo setoso. Lixpl. 1. c. p. 5ı.-R. hispida. Pronv. Nom. p. 34.-Idem. ThoRY. Prodr.

R. Var. D. Prlosa. Pumila; foliis acutis, infrà pilosis.

R. Var. E. Terbinata. Pumila; fructu turbinato.

li. Var. F. PaLlas. Elatior; armis subxqualibus, confertis. $L_{1 \text { Nol. }}$. cit. p. 5 r.

R. Pimpinellifolia. Pazl. Fioss. 6a. t. 75. - Redont. Fios. 1, 84.

R. Alläica. Willo. Enum. 5q́3.

R. Var. G. Rossica. Elatior; aculeis longis, gracillimis.

f. Var. $\Lambda$. Istandica. Elatior; aculeis maximis, falcatis. 
K. Iybernica. Hoon в Irland. in app.

h. Var. I. Sanguisorbefolia. Elatior; fuliolis $9^{-11}$, oblongis; fructu depresso, globoso. Norsette. Cat-Doon. Canti. ed. 8. 169.

h. Var. I. Redutea. Llatior; armis confertissimis; pedunculis setosis; foliis glaucis; floribus geminatis. Pronv. Nom. p. 2 r.-R. Reduteana. Trony. Prod. p. 43.

Sc trouve en abondance dans toute l"Europe, sur les montagne's boisées et aussi sur celles qui ivoisinent la mer, ainsi que sur le Caucase (Bubersteiv); les variétés D, E en Islandle (Hookfr); la varićté F en Pussie (Pallas); var. H en Irlande (Ilooker); var. I observée cliez M. Noisette, à Paris; var. L., observée chez M. Thory, d Clamart. ( $V$.v. cult. et s. sp.)-OEs. Cétte espéce varie beaucoup pour la dimension et la surface du pédoneule et du fruit. Les branches sont tantôt nues ou presque nues, tortueuses, minces, élancées, couvertes d'aiguillons pressés suitnut dans les jeunes pousses. La var. D offre plusieurs aberrations. Lind. loc. cit.

Arbuste nain, compacte, sombre (quelquefois rougeâtre); râcines traçantes. Branches courtes, raides, très-divisces, couvertes d'aiguillons épais, inégaux, et de soies; quelques aiguillons courbés en laux. Feuilles rapprochées en faisceaux sans aucune pubescence. Stipules ou étroites ou délitées, d'égale largeur ou à peu près. Pétioles soyeux et aiguillomés. Fotioles, cnviron 7 , d'un rert clair, aplaties, dentées simplement, orbiculaires ou à pen près. Fleurs solitaires, sans bractées, cyathiformes (en bassin). Pédoncules nus ou garnis de soies ou de glandes, comme les sépales qui sont courtes et entières. Tube nu, ovale, on presque rond. Pélales émarginés, concaves. Disque sans épaisseur. Styles velus, distincts. Fruit ovale ou presque rond, noir on presque foncé, couronné par les séjales conniventes ou quelquefois étalées.

J'ai déjì dit pourquoi je ne pouvais être d'accord arec le docteur Afzelius, qui considére le $R$. spinosissima prima et vera de Linnée comme le cinnamomea, et je ne suis pas plus de l'avis de $\mathbf{H}$. Woods, qui veut que le pimpinellifolia de l'Jerbier de Linnée soit le rubella. Je n'hésite pas ì me prononcer, comme s. J. Smith l'a fait depuis long-temps, en laveur du spinosissima var. A. L'opinion de Schranck, qui en fait le $R$. collina de cet anteur, ne vaut pas mieux, quoiqu'elle repose sur l'autorité de Rau. Le spinosissima de Gorter semble être un cinnamomea, tandis que la plante ainsi nomınée par Hermann semble un spinosissima, à rajson de ses feuilles orbiculaires et de ses pédoneules soyeux. Les figures de Bulliard et de la Flore danoise ne sunt pas sufisamment caractérisées. 
Nutre spinosissima ne samait être confondu avec le vimisea et le grandiflora. Les rameaux droits, vigoureux, et les forts atguillens du premier le distingraent suffisamment. Il en est de même des soies nombreuses parmi lesquelles se trouvint les aiguillons dims le second.

Lit variécí li reversa a les aiguillons fitbles et réfléchis; les feuilles sont trés-glatques et la plante se couvre au printernps d'une grande quantité de fleurs blanches comme la neige. bien des auteurs en feraient une espéce distincte. Son lieu natal est inconnu, à moins que le spinosissima de Jacqui!n, trouvé indigène en Autriche et figuré dans ses Fragmenta, ne soit le même; il en differe peu si ce n'est par les aignuillons qui sont horizontaux.

Les variétés platycarpe, iilosa et turbinata ne sont connues que par des échantillans recueillis en Islande par mon ami M. Hooker. Leurs caractères déterminent suffisamment leurs différences respectives. Le pilosa ne párâit se distinguer de l'involuta que par les dents simples de ses feuilles. Il est zéanmoins très-différent.

Le Pallasii croît dans les plaines élevíes et dans les pentes raitles de la partie septentrionale des monts Altä̈ques qui s'étendent jusqu'en Sibérie. Son habitude vigoureuse et la dimension égale de ses aiguillons sont ses principaux traits.

Le rossica a ses aiguillons longs et faibles. Il se Irouve dans le vaste herbier de S. J. Smith.

L'islandica est le seul rosier trouvé en Islande. Ses raIneaux forts et vigoureux ont induit M. Hooker en erreur lorsqu'il en a fait un hibernica qui, je crois, n'a jamais été découvert aillcurs qu'anx environs de Belfast.

Le sanguisorbcfolia a une apparence différente des autres variétés. Ses pédoncules sont très-courts, les folioles plus nombreuses (de 7 ù 11 ). Sa patrie esi inconnue.

"Le reduteana de II. Thory me paraît une variété du spi. nosissima. On le prendrait pour un rubrifolia a une certaine distance. Ses fleur's sont quelquefois géminées : ce qui est remarquable dans l'especce."

Aucune espèce n'est aussi embarrassante que celle-ci, parce que, croissant dans une grande partie de l'Europe et de l'Asie, elle offre des nuances très-variées. La culture a achevé de l'écarter du type originaire; mais on le retrouve à Fontainebleau, en Écosse et ailleurs. 


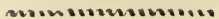 \\ 2Se. ESPECE. - ROSA GRANDIFLORA.}

R. Setis ramorum nullis; aculeis, subxqualibus, distantibus; foliolis planis, impuhibus, simpliciter serralis. LuNov. Nom. p. 53. c. fig.

li. Grandiflora. Provv. Somm. p. 22.

R Pimpinellifolia. Bus. Taur.-Cauc. 2. 39\%.

Se trouve en Sibérie (Hovт.); sur les parties sous-alpines et stériles du Caucase (Buterstein). ( $V$. s. sp. Herb. Lyell, Subine.)

C'est particulièrement ì la sollicitation de H. Sabine que je me suis décilé à séparer cette plante du spinosissima. $\mathrm{Hls}$ different presque de ia même maniere que l’innovata et le Sabini, excepté que ce dernier est plus gigantesque, même dans sa varicté $\mathbf{B}$, que celui-ci. Les caractères différentiels entre notre plante et le spinosissima sont, de plus grandes flcurs, -l'absence des soies parmi les aiguillons de ses jeunes rameaux, caractères qui paraissent ici constans, quoique je ne les aie pas admis en unissant le Sabini avec le doniana; c'est pourtant une plante trop remarquable pour qu'il n'en soit pas lait menion, et si par la suite elle devait être réunie au spinosissina, elle en serait toujours une des plus notables variétés. Je ne doute pas, d'après les détails de Buberstein, que son pimpinellifolia ne soit eelui-ci, surtout en observant qu'il le sépare lu spinosissima, ce.qu'un bolaniste aussi exact que lui n'aurait pas frit, s'il n'avait pas aperçu de différence entre eux.

" Fidéle à la loi que je me suis imposée de n'admettre aucune espèce qui n'est fondée que sur une description écrite, je passe sous silence le $R$. nantinensis de Loureiro jusqu'à ce que cette plante nous soit parvenue soit vivante soit en spécimen bien conservé. „

\section{$29^{\mathrm{e}}$ ESPÈCE. - ROSA MYRIACANTHA.}

R. Armis inæqualibus, majoribus, pugioniformibus; foliis glandulosis, impubibas, orbiculatis. Linol. Mon. p. 55 cum fig.

R. Alyrincantha. Prosv. Somm. p. 22.

R. Parvifolia. P Pll. Ross. 62.

R. Provincialis. Buв. Taur.-Caue. 1. 39G.

R. Myriacantha. Defand. Fl. fr. 4.439 .

Se trouve à Osset, dans les buissons (Pallas); en Daurdhiné (Dr- 
caxdoulz); pris Hontpellier (RLQuies). ( $\zeta^{\prime}$. s. sp. in Lerb. Hoorke, LANBERT.)

Petit arbuste rabnugri ; avec des rameaux simples, presque drnits, brunâtres et défendus par des chiguillons laibles, nombreux, inégnux et dioits. Ils sont mêlés de soies. Feuiltes porlées vêrs l'extréuité des romeaux et sans pubescence. Stipules étroites, grlandulenses au revers. Pétioles glanduleux et soyeux, garnis de quelques pelits aiguillons droits et épars. Folioles de 5 i 7 , elliptiques ou orbiculaires, doublement dentées, glanduleuses et rouillées en dessous. Fleurs solitaires, en coupe, parmi les feuilles, et inunies de bractées. Pédoncules et calices garnis de glandes et de soies nombreuses excepté la partie supérieure du tube qui est globuleux. Sépales réfléchies apress la floraison, et plus longues que le fruit avant la maturité. Disque un peu relevé; les parties saillantes des styles et stigmates un peu velues.

Cette petite plante n'a été trouvée jusqu'à prẻsent que dans le Midi de la France, à moins que le synonyure cité par Pallas ne lui appartienne. Toutelois ce qu'il en dit est trop incomplet pour nous décider affirınativement. Les habitudes différentes sont toujours une objection de fait. Les glandes de ses feuilles suffisent pour empêcher qu'on ne la conlonde avec le spinosissima nain, dont elle porte quelques caractères.

Le provincialis de Buberstein répond précisément à notre plante, et confirme mon idée que le synonyme de Pallas s'y rapporte également.

Nous avons dans nos pépinières un micranthas qui approche de celui-ci; mais les aiguillons ne sont ni aussi longs ni aussi nombreux. Peut-être a-t-il été considérablement altéré par la culture; il ne faut pas confondre cette petite plante avec le micrantha de Smith, qui est un rubiginosa.

\section{0\%. ESPĖCE. - ROSA INVOLUTA.}

R. $\Lambda$ rmis valdè inæqualibus, confertissimis; foliolis duplò serratis, pubescentibus; petalis convolutis; fructu aculeato. LiNDL. Mon. p. 56.

R. Spinosissima. MoensH. Meth, 687 .

R. Involuta. ENg. Eot. t. 2068.-A1т. Kew. ed. all. 3. 26o. - S in Rees in 1. - Woods. in Act. linn. 12. - Pronv. Somm, p. 23.

R. Nivalis. Doos. Cant. ed. 8 . 170 .

Se trouve sur les montagnes de l'Écosse (WALKER). ( $V$. v. cult. et s. sp.)

De 2 à 3 pieds de haut, touffu, d'un gris rougeâtre. Bran- 
ches peu dirisées, droites, arec des aiguillons et des soies nombreuses; les aiguillons forts, droits et inégaux. Feuilles serrées qui répandent une odeur de térébenthine quand on les froisse. Stipules étroites, quelquefois concaves, aiguës, non dentelées, mais frangées de glandes. Pétioles velus, glanduleux et soyeux, mêlés de quelques aiguillons sétiformes. Folioles de 5 à 7 , concaves, ovales, aiguës ou obtuses à double dentelure, nues en dessus et presque opaques, velues en dessous avec quelques glandes pâles à peine perceptibles à la surface. Fleurs solitaires, sans bractées, rouges et blanches. $P \dot{E}$ doncute nu.Tube du calice ovale, presque sphérique. Sépales hérissées d'aiguillons sétiforthes et de glandes visqueuses. $P \dot{E}$ tales obcordiformes (presqu'en cœur). Disque peu élevé.Fruit couronné par les sépales convergentes avant la maturation.

On est redevable de la découverte de cette plante à $\mathbf{~ I I}$. Walker qui l'a trouvée dans lcs montagnes de l'Écosse, et il ne paraît pas qu'elle ait été vue ailleurs. Du moins tous les échantillons que j'ai vus étiquetés Rosa involuta étaient le Sabini ou sa variélé doniana. Il n'est pas aisé, comme l'on sait, de déterminer des caractères distinctifs sur des plantes déssécliées, et si diflérentes en pleine végétation. Le R. involuta est un arbuste sombre, avec des pétales roulés et des aiguillons tries-rapprochés, presque nus en dessus, et son fruit ue mûrit pas dans l'état cultivé. Le Sabini, au contraire, est une plante élevée de 5 à 10 pieds, et quand ses aiguillons sont entremêlés de soies, les plus grands sont en faux, ce qui n'arrive point lorsqu'ils en sont dépourvus. Les feuilles sont velues des deux côtés et chargées quelquefois d'une poussière blanchâtre; son liruit marit très-bien dans nos jardins.

"Nota. Le $R$. involuta a fleuri à Versailles en 1825 . Malgré quelques rapports généraux de physionomie, on ne peut le confondre avec le spinosissima. D

Je n'ai pas adopté le $R$. reversa, qui n'est connu jusqu'a présent que par la description qu'en a laite Waldestein. Voicila phrase de Lindley:

R. Armis setaceis; subæqualibus, reflexis; foliolis duplicato-serratis, pubescentibus; fructu hispido. Lisd. Mon. p. 57.

1. Rever'sa. WaLDest. et KitalB. Hung. 3. 293 . t. 26 f́.

Se trouve dans les endroits pierreux des montagnes de Natra. W. et $\mathrm{K}$. 
J'indiquerai de même le marginata de Walrave.

R. Pumila. Ramis tortuosis, junioribus pruinosis ; foliolis ovatis, cordatis, triplo-serratis, slaberrimis; sepalis muricatis. LINDL. Non. p. 58 .

R. MIarginata. WALLR. An. bot. 68 .

Se trouve dans les champs, pris de Beunstadt.

\section{$3 I^{\circ}$. ESPECE. - ROSA SABINI.}

R. Setis raris aculeisque inequalibus, distantibus; foliolis dupli- erratis, tomentosis; sep.lis compositis. Livor. Mon. 1. 59 .

R. Subini. Woods. in Act. linn. 12. 188. - Pronv, Somm. p. 23.

R. Involuta. Winch. Ess. geogr. 41 .

Var. \&. Donsana. Sctis subnullis; aculeis rectiusculis.

K. Dontana. Woovs. 1. c. 12.185.

Se tronve ıu nord de l'Angleterre; la variété $f$ dans le Sussex (BоRR.r). ( $\mathscr{V}$. v. cult. et s. sp.)

Arbuste de 8 à 10 pieds de haut. Branches droites, brunfoncé, armées d'aiguillons distans, cuurbés un liutux, et de quelques soies. Feuilles grises, distantes. Stipules étroites, firangées de glandes. Pétioles colonneux, glandulitres et armés de quelques petits aiguillons. Folioles de 5 à 7 , oviales, à scrrature double, plates, velues des 2 côtés, un peu glandulaires en dessous. Flcurs ordinairement solitaires, quelquefois ramassées. Pédoncules ef Calice très-hispides. Tube rond. Sípales composées. Fruit rond,écarlate, couvert de soies.

D'après des échantillons de M. Winch, je me suis convain. cu que celui-ci est son involuta. C'est une charmante plante, et comme elle l'einporte de beaucoup sur les espèces anglaises. Il. Woods a bien fait de la dédier à M. Sabine, notre ami commun.

Il diffiere de l'involuta par son habilude robuste et ses forts aiguillons. Les péiloncules sont solitaires on aggrégés, et, dans ce dernier cas, garnis de bractées. Les sépalessont anssi composées. Il tient si précisément l'iutermédicire entre cette tribu et la suicante, qu'on pourrait le placer indifféremment dans l'une ou dans l'autre; mais, comme plante indigène et alliée à l'involuta, j'ai préléré le placer dans celte tribu, malgré ses sépaltes diviséeset son disque quelquefois épaissi.

La variété $\beta$ doniana est moins éleyée; elle a des aiguillons droits, suns soies sur les rameanx. Cie dernier pourrait être aussi une production du tomentosa moltis?

"La varieté domiana a neuri a Versailles en 1823 . Je cultive également le Sabini que j'avais perdu. 


\section{VIe. 'TRIDU. - CENTIFOLIAE.}

Caractères particuliers. - Setigeræ ; armis difiormibus. Folia oblonga vel urati, rugosa. Discus incrassatus, faucem claudens; sepala composita.

Obs. - Celle division renferme la parlie la plus intéressante du genre pour les amateurs de Flore. 11 est probable que les roses les plus anciennes dont il ait été fait mention apparliennent à quelques-unes de ces espèces, mais auxquelies les espèces particulieres, comme ielle de Cyréne ou du mont Pangeus, doivont être rapportées : il cst mainienant trop tard pour entreprendre ces recherches. Un me permellra pourtant de conjecturer que toutes descendent d'unc souche commune, et que par une longue culture elles ont été conduites à prendre les apparences sur lesquelles les botanisles ont pu déterminer les caractères différentiels.

L'essence de roses. qui est un objet de commerce si important, s'obtient indifléremment de toutes ces roses, daus le laboratoire de Florence élabli dans un ancien couvent, ou de quelques espèces particulières connues dans l'Inde. D'après des échantillons del'herbier de I.ambert, apportés de Ghispere parle c. Hardwicke, il praraîl que le $R$.damascena y est exclusivement employé nour la fabrication de l'huile essentielle, et les l'ersans en emploient une espece gue Kæmpfer appelle chirasensis, parce qu'elle croît dans les environs de Chiras, de preférenco à loute autre : celle-ci peut être la même, comme j'aurai sans doute oceasion de l'expliquer. Il est bien connu que l'essence de roses de différontus contrées a des qualités de lurce et d'énergie bien différentes; que celle qui vient de la Turquie est toujours préfírée ; et je suis porté à penser que le $R$. moschata pent êtrc employé seul ou mélangé a vec d'autres, particulièrencent à Hogariur, où, comme je l'apprends du docteur Schuter, il s'en fabrique leaucoup mais d'une qualité inlérieure.

Les innomiurabies variétés cullivéts dans nos jardins se rapportent aux quatre ou cinq espèces suivantes. Comme il n'entre point dans uon plan de parler de celles qui n'intéressent point les botunistes, je laisse volontiers aux soins de 11. Subine de decrive le raritís doublez, aliendu qu'il se 


$$
6 ;
$$

ponose de donner d̀ cet égard les plus ampli's détails (1). II 111 suffira, en altemlant, de tracer les caracteres distinetifs las espèces de cette tribu, avant de donner la description particulière de chacune d'elles.

Ces espices sont toutes soyenses, en quoi elles se distiuguent des divisions suivantes. Leur disque charnu et leurs sépales diviséesservent à les séparer des précédentes. I.e port ile ces plantes, la dimension de leurs fleurs, et la nature de leurs glandes pédicellées, empêchent qu'on ne les contonde avec les rubiginosa et les tribus suivanles.

\section{2\%. ESPECCE. ROSA DAMASCENA.}

R. Armis inæqualibus, majoribus falcatis; sepalis reflexis; fructu elongato. Lind. Mon. G2.

K. Damascena. MiLl. Dict. n. 15. - Doro1. Harbk. 2. 36ł.- iVILLD? Sp. 2. $1072 .-A_{1 x}$ Kew. ed. alt. 3. 263.

Rosier de Damas. Bosc. Dict. d'agr. t. 11. $25 \%$.

R. Bifera. Poin. Enc. 6. 2;6.-Pers. Syn. 2. 49. - Piedodt. Ros. I. 137. t. 53. - Prosv. Nnm. p. 79 .

R. Cenlifolia. Var. ל bifera. Pork. Fnc.

R. Calendarum. Monch. Hausv. - Bork. Holz. 33o. - Rorss. Pios. t. 8. Var. 6. Purlundica. Pansv. Noin. p. 79 .

R. Gallique, variété de Portland. Bosc. Dict. d'agr. 11. 332.

Se trouve en Syrie (Smirn); la variélé 6 vient d'Inglcsore. ( $V$. v cult. et s. sp.)

"Quoique jepersiste à distinguer ce rosier du suivant, comme l'ont fiat, d'aprés Miller, MII. Bosc et Dumont de Courset, je conviens que j'ai commi; une erreur de urın, cn ôtant dans ura Nomenclature de 18,8 au véritalsle damascena celui que les premiers botinisles lui avaient dumé ".

“D'après de nouvelles observalions, il paraît que ce beau rosier, qui nous vient de l'Orient, était comn et célébré par les anciens. Sprengel pense qu'il pourrait être celui que Virgi'e cite dans ses Georgiques (bifera rosaria P'asti), quoique les bolanistes ne l'aient pas encore relrouvé depuis aux environs de Pæstum. S. Janres Smith croit qu'il a élé introduit en Europe par le comte de Brie, compagnon d'armes de St. Louis,

(1) "Depuis quelques annécs, les essais multipliés die nos jardinicl's ont fait obtenir des variétés doubles d'un plus grand nombrc d'espèces. C'est ce dont on pourra se convaincre en lisant l'appendix qui termine cet ouvrage." 
à son retour d'Égypte. Ainsi ces chevaliers auraient mbelli nos parterres de leur plus bel ornement, comme ils auraient auginenté le nombre de nos fruits et de nos légumes; s'il est. vrai que nous leur devons la prune de Damas et l'échalotte (allium ascalonicum). Mais Nicolas Monardi, qui a fait un assez long séjour en Syrie, ne fait dater leur introtluction en Europe que de 15,5 . La description qu'il fait du $R$. damascena se rapporte à notre plante. Elle acquiert en Syrie de plus grandes dimensions; ses fleurs sont plus nombreuses et d'un rouge pâle.»

"Je remarquerai qu'il s'élère quelquefois ì une grande hauteur. J'en possède un non gruffé qui a quinze pieds d'élévation $v$.

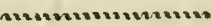

\section{ESPECE. - ROSA BELGI CA.}

R. Armis incequalibus, majoribus falcatis; sepalis reflexis; fructu ovato, peduuculo longiore. Prosv. Somm. p. 25

R. Belgica. Hilu. Dict. n. 17.

Rosier beigique. Dom. Cours. Bot. cult. III. p. 3/7. - Bosc. Dict. d'agr. II. p. 255.

R. Damascena. Dorol. Harbk. 2. p. $36_{9}$ - Redort. Pos. 121. t. 45.Pronv. Nom. p. $7^{3}$.

R. Alba. Var. damascena. Polr. Enc. 24 .

Rose de Puteaux, des env. de Paris.

Linné conlondait le damascena et le centifotia, parce qu'en effet ils ont des rapports communs, et qu'à une époque déjà loin de nous, on avait pu croire qu'ils sortaient l'un de l'autre; mais la forme des fleurs, celle des fruits, l'aspect général de ces plantes motivaient une distinction. Quelques auteurs, et $\mathbf{H}$. Lindley lui-même, ne font qu'une espèce du R. damascence et du belgica. M. Poiret les a séparés avec raison, mais il en a formé dcux varićtés, l'une du centifolia, l'autre de l'alba. Cependant il est facile de se convaiucre, d'après une observation exacte, que ces deux rosit rs sont des espèces très-distinctes. Le $R$. belgica offre un luisson touffu, beancoup moins élevé que le dumascena; des rameaux et pétioles inoins garnis d'aiguillons; des fulioles ovales, aiguës, dentées, tomentenses, et plus ou moins blanchâtres en dessous. Des fletr's terminales, souvent at nombre de 10 d 12 sur le même corymbe, moins grandes que dans le damascena, portées sur de longs pédoncules hispides, écartés les uns des autres; ses fruits sont ovales et renflés (ovatis, turgidis), ce 
qui les distingue notablement des prenières (seminibus infundibutiformibus, Porr.): les sépales sont pinnées et rétléchies, mais les corymbes sont érigés, ce qui les sépare de l'espèce suivante.

C'est ce rosier que les habitans de Puteaux cultivent au pied du mont Valerien pour les prarlumeur's de Paris. Je pense qu'il tire sun origine des pays mérirlionaux, d'où il aura été transporté en France ct en Angletcre. Les jardiniers en ont obtenu de belles variétés, et la rose d'Yorck et Lancastre, citee par Miller dans son Dictionnaire, est une des plus remarquables.

\section{3 f. ESPECE. - ROSA CENTIFOLIA.}

R. Armis inaqualibus, majoribus falcatis; foliolis glanduloso-ciliatis; floribus cernuis; calycibus viscosis; fructu oblongo. - Linde. Mon. p. 64 .

R. n. l. Liss. Cliff. 191.

R. Centifolia. Lins. Sp. gof-Dorol. Harbk. 2. 36j.-Bull. Par. t. 275. - Lour. Coch. t. 323 ?-Roess. Ros. t. 1. - Bes. Taur.-Cauc. 1. 397. - Rav. Enum. iog. - Redodt. Ros. 1. 25. t. 1. - 37. t. 7. -72 .

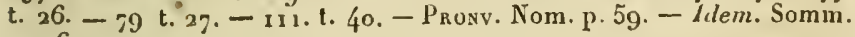
p. 26.

R. Provincialis. Miller. Dict. n. 18. - Doror. Harbk. 2. 349.Willo. Sp. 2. 1070. - Pers. Syn. 2. 48. - A IT. Kew. ed. alt. 3. 261.GMel. Bad.-Als. 2, 429.-Smith. in hiees in l.

R. Polranthos. Roess. Ros. t. 25.

R. Caryophyllea. Poir. Enc. 6. $2 ; 6$.

R. Inguiculata. Desf. Cat. 175 .

$R$. Virians. Ронг. Bohem. 2 izi.

Var. $\beta$. Muscosa. Calycibus pedunculisque muscosis.

R. Riulira plena, spinosissima; pedunculo muscoso. MILL. Ic. 221 . f. I. R. Muscosa. Mill. Dict. n. 22. - Doror. Harbk. 2. 368. - Wrín. Sp. 2. $1074 .-$ Lour. t. 14 - Roess. Ros. t. 6. - P $\mathrm{E}_{\mathrm{R}}$ S Syn. 2. 49. Ait. Kew. ed. alt. 3. 26 \% - Ker. Regist. tt. 53-102. - R̈enout. Ros. I. 39. t. 8. -4 i. t. 9.8 t. $3 \mathrm{r}$.

R. Provincialis b. Smitr. in Rees in 1.

Var. C. Pomponia. Omuibus partibus minor.

K. Centifolia minor. Rers. Rios.ti. 20. 37 .

R. Divionensis. Roess. 1. c. t. 2 '

R. Pomponia. Dec. Fl. fr. 4. 433.-Redodt. lios. 1. 65. t. 21.

R. Burgundiaca. Pers. Syn. 2. 48.

R. Provincialis $\gamma$. Smitu. in Rees in 1 .

$R$. Centifolia $\tau$. Redout. Ros. 1. I13. t. 4r.

Var. D. Bipinvata. Foliis hipinnatis.

R. Centifolia bipinnala. Pers. Syn. 2. 48. - Rejout. Ros. 2. It. t. 4.

Se trouve dans les forêts de la partie orientale du Caucase ( $\mathrm{Bu}-$ MESTEIRI). 
Ce beau ruoitr a beaucoup de rapports arec le précédent; unais il peut en être distingué par sés sépales, qui ne sont réflechies en ancun temps; ses fleurs très-doubles et ses larges pétales, dont la disposition a lait que l'on nommait cette !)lante en Angleterre rose-chou, ce que Linné a désigné d'une limnière plus élengante en disallt: flos quasi tornatus. Son fruit est chblong vu presque rond, mais jamais allongé (1). Le R. cent leuiltes se distingue aussi des suivans par ses fleurs penchées, soil port habituel, stes rameaux diffus et rehrousses (retrofracti). Ce rosier peut se inultiplier de boutures, unais pas allssi facilement que le dumascena et le gallica.

S. Jauses smith est porté a croire avec plusieurs autses que ce rosier est originaire du uidi de l'Lurope; mais rien de ce qui a été dit à cet égard ne pouvant faire auturité pour désigner le lieu natal d'une plante aussi généralement cultivée, c'est pourquoi je prélère placer son origrine en Asie, parce que Buberstein l'a trouvé dans l'état sauvage sur le côté oriental du mont Caucase, d'où il aura vraiscunblablement passé dans nos jardins. Peut-être la célébre rose de Chiras, dont Kæmpfer fait un si grand éloge, n'est que celle-ci ou le damascena : nous ne pouvons que le conjecturer; au reste, les fleurs de la rose de Chiras sont employées à la distillation ainsi que celles du gallica.

Polh, dans sa Flora bohemica, a considérí les $R$. provincialis et gallica comme varietés l'un de l'autre. Je suis, plus disposé à adopter l'opinion de Borckhansen et de quelques buianistes francais, en regardant le provincialis de Miller et le centifolia de Limans comme la même espéce. C'est pourtant ce que l'inspection de l'Herbier de Linié ne proure pas; il faut ayoir recours a d'autres moyens pour confirmer la justesse de cette opinion.

" Qu'un use permette de passer une longue discussion d'après laquelle on pourritit douter que Linne ait eu en vue le véritablé centifotia dans la première édition de l'Hortus cliffortianus, et il y a toute apparence que le centifolia et le galtica ont été conlondus ensemble. Le $R$. officinatis des Français est bien cclui de Provins; mais l'erreur de Linaé, réparée depuis dans la secunde édition du Sp. plant., vient de Miller; elle a été suivie par quelques botanistes allemands.

(1) Cependant les fruits da R. inguiculata ou caryophyllea qui es: notre rose oxiliet, sont sensiblement ovales et aliovges vers ltur ex1 rémilé suycéricurs. 
I. rose monsseuse est une pure variélí qui a bmites las appareures in provins (1). MII. Lce et Kennerly posactent une phante yn produit l'un et l'antro sur la même tige, et s. Jumes Smith a appris en Italic que la mousse qui recouvre le calice et le pédoncule de celle piante disparaissanit bientôt daus cet climat.

Lat val. C pomponice, gue l'on a siggrulierement confondue avec la ruse de Bourgorne, est plus petite daus tomtes ses parties, ct la variéti a feuilles le céleri (bipinnata) des jardins francalis est une singulière manstrmusité. J'ai ru la II) êmı: diffirmicé sur un canina dıns le jardinde M. Sabine.

* Il existe une singulièro varictí du cencifolia chez madame la contesse de Chimmois, pres de Sezanne en Brie. Celte plante ne sielive qu'a un pied au plus; clle irace beaucoup, et ses fleurs duubles sont d'une proportion mnyenuc. On en fait des lourdures dans les jardins de cette dame, qui en a envoyé à Versailles où je l'ai observée. „

\section{ESPECE. - ROSA PIOVINCIALIS.}

Ћ. Armis ramorum inimqualibus, sparsis, ratunion!n subuullis; foliolis oblongis, arguté duplo-serratis; peisulis aculeatis; tloribus ercetis; sepalis sefleris; fructu ovato. Pron. înm. p 43.-Ldentiomn. p. 26.

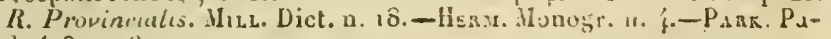
r.il. $413 . \mathrm{n}$ (i.

Rose ile Pruvence. Bosc. Dict. d'agr. t. 1 r. - Lawk. Ros. A, 32.

R. Centifolict. Linol. Mon. p. 6$\}$.

K. Gallica. V.A. Poir. Enc. 6. p. 278 .

Var. $\beta$ Incarvata. Floribus pallidis.

R. Incarnata. Mill. Dict. 11. 19- - PARK. Parad.412. 11. a.

Blush Provence rose. Lawr. Rios. n. 1.

Se trouve en prorence (hiнтуі). ( $/$. r.cuit. )

Il est possible que ce rosier, qui vient du midi, ait ajouté a la confision que les anciens botanistes ont prouluite cntre le K. centifolia et le gailica, avee lesquels il a taut de rapports. Lst-ce notre plante on le damascena que Beslier a appeli R. milesia dans son Hortus eysteucensis, et que l'on a pu prendre pour le centifolia, ce yui a lait croire à quelrues anteurs que ce dernier iatait origrinare rlu sad? Quni qu'il en soit, si le provincialis, qui a pu être produit daus des temps bien éloignés du croisement du cencifotia et du gallica,

(I) II y a peut-être ici une erreur, car la rose inousseuse est lien "1̄ centifolia, ct quoiqu'elle soit originaire de Provence, suivant la locteur Vartyo, on re peut la confondre arec le $k$. provineicáis. 
devait être réduit au titre de variété, je ne balancerais pas á le joindre au gallica, dont ses feuilles non glanduleuses et ses fleurs érigèes le rapprochent; mais il peut être considéré coınme une espèce intermédiaire.

Ses branches sont droites, armées de peu d'aiguillons; les rameaux en sont privés, mais les pélioles en portent un petit nombre. Les feuilles sont plus graudes que celles du gallica; les fotiotes larges, pointues; les dentelures doubles, trèsaiguës; les rameaux sont tcrminés par des rorymbis de 3 ou 4 fleurs rolges ou carnées, grundes, semi-iluubles; les pédoncules et tube du calice peu glanıluleux; les sépates tièsdivisées et réfléchies, ce qui le sépare encore phıs du gatlica. Cet arluste, que l'on cultivait à l'école du Jardin ruyal des Plantes de Paris, où il n'existe plus, s'élève quelquelus à 8 ou 9 pieds de hauteur. Son croisement avec le gallica a produit dans nos pépinières des variétés hybrides connues sous le nom d'agathes, probablement parce que leur corolle extrêmement double est marqué d'une ou plusirurs taches foncées, comme on le remarque dans les quartz qui portent ce nom.

Un rosier nain qui porte dans les pépinières le nom de rose de Champagne, de jompon des Alpes, ou cglanteria pumila, semblerait une variété du provincialis par ses feuilles à serratures aigriës et ses sépales dirisées et cotnnnenses dans toute la parie extérieure, et je ne remarque aucune pubescence sur notre plante. On ne peut pas plus le confondre arec la varićté pomponia et le $R$. parvifolia que le provincialis avec ses deux roisins.

\section{ESPËCE. - ROSA GALLICA.}

R. Armis subxcqualibus, conformibus, debilihus; foliolis rigidis, ellipticis; floribus erectis; sepalis ovalis; fructu subgloboso. LiNDz. Mon. p. 68.

R. Rubra, etc. BADH. Hist. 2. 24 .

R. n. 3. Liss. H. cliff. 19 t.

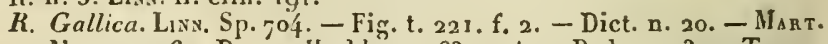

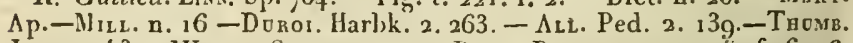
Jap. 214 ? - Willn. Sp. 2. roj1. - Roess. Ros. tt. I5-22-25. f. 6.26.

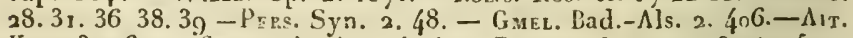
Kew. 3. 262. - Sм1тн. in liees in 1. - Redodt. hos. 1. 73. t. $25 .-$ 135. t. 52. - 2. 17. t. 7. -19 . t. 8. 10. - Prosv. Nom. p. $4 \mathrm{r}-1 d$. Somm. p. 27 .

R. Centifolia. Milz. Dict. n. 11.-Willd. Sp. 2. 1071:-Pers. Syn. 2. 48 . 


\section{3}

Roster de Provins. Reg. Bot.

R. Sylvutica. Gatт. Montaub. 9'.

R. Rubra. Las. Fl. fr. 3. 130.

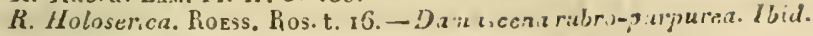

t. 18 .

R. Belgica. Brat. Lus. 1. 338.

IR. Blanelu. Brat Ioc. cit?

R. Cuprea. JacQ. Fragin. 3i. t. 3' f. 4.

Var. q. Pumila. Horibus simplicibus; ladicibus repentibus. LiNol.

R. Punci'a etc. Bavh. Hist. 235

K. Pumila. Linn. Suppl. $262 .-$ Jare. Austr. 2. 59 . 1. $19^{8}$ - Alu.

Pcd. 2. ŕfo. - Wicho. 2. 1072. - Pres Syn. 2. 49. - Bub. Tiall.Canc. 1. 397.-Arr. Kew. 3. 263 - Puill. Boliem. 2. 172. - Vullu. Canc. $150 .-$ Smith. in Rees in $1 .-R_{A u}$. Enum. 112. - Provv. Nom. p. 90 .

K. Nain d'Autrichs. Mart. Ap.-Dilill. 19.

R. 110 f. I $_{\text {ALL. Helv. }}$

R. Repens. Miunch. Hausv 5. 28 r.

R. ilispida. MuUnc1r. Joc. cit.

R. Austriaca. Chantz. Austr. 86.-Poluch. Palat. 5o.

K. Olympica. Doos. Cant. ed. 8. 170.

Var. $\gamma$. Arvina. Foliis utrinqué nudis.

R. Arvina. Kкоск. Siles. 2. 156. - Rav. Enum. 106.

Se tronve dans les haics, près de Montauban (Gatteresu); dans les buissons, prés de Wurtzbourg (IVIBeL); var. b prés de Genève ( $\Lambda_{L}$ Lovi); la var. $\gamma$ sur les bords des champs, près de Retbbaclı (Ridu). ( $V$. v. cult. et s. sp.)

Puisque le pumila de Jacquin doit être considéré comme le type de cette espèce dans l'état sauvage, il aurait peut-être mieux valu le placer comme variété; cependant le nom bien connu de gallica doit avoir été donné í un autre dont le gisement s'étend ì peine hors du lien ou il se tronve indigene.

La Suisse et l'Autriche le produisent (le pumila) en abondance, et toutefois Buberstein l'a trouvé en Asie. Ru nous dit que daus les environs de Wurzbourg il crô̂t en telle quantité, qu'il nuit aux gruines céríules par ses racines traçantes, comme le rubus ccesius. Il est plus connu dans nos jardins sous le nown de $R$. olympica que Doon lui a donné, tandis que le nom pumila est improprement appliqué au majalis.

Les nombreuses variétésloubles, connues sous les noms de géant, velours, évéque, sont de la plus grande heauté(r), et elles seraient sans pareilles dans le régne végétal, si elles

(1) "Je re rois plus la rose géant sur nos catalogues, pas pline que la beauté arientale, la peinture, la donceur et antres variétés y"ii sont. signalées dans les collections de flarlem. Il y aura bientôt fard de noms, qu'il faudra s'en tenir aux numéros." 
étaient douées du parfum qui caractérise des espèces moin: brillantes. La plus magnifique de toutes est la rose de Toscane, dont M. Édouard Sydenham a donné une excellente firnure qui ne tardera pas à paraître dans le Bolanical register.

La variété o arvina de la Flore silésienne de Krocker ditfère tris-peu de l'espéce, ainsi que Rau lui-même l'assure, si ce n'est que le tube du calice et les lolioles sont glibres.

J'ai dejà dit que le $R$. galtica avait beancoup de rapport arec le centifolia; mais ses pédoncules courts et rigides, l'absence de grands aiguillons, ses pétales plus petits et sés sepales ovales, le firent toujours distinguer. Son ensemble est plus touffu, et ses dimensions moilıs grandes (à quelques exceptions près ). Ses l'euilles sont presque glanduleuses, caractère très-marqué dans le centifotia, et souvent les serratures sont i peine visibles.

Le $R$. galtica de Forsckhat, qu'il dit croître à Constantinople à la hauteur des maisons, el qui porte des fleurs blanches, ne peut pas être celui-ci. Le prendrait-il pour le moschata, qu'on y cultive?

\section{$37^{\circ}$. - ESP'ECE. ROSA PARVIFOLIA.}

R. Nana; armis subxqualibus ; foliolis rigidis, ovatis, acutis, argutè serratis; sepalis ovatis. Lind. Non. p. jo.

fi. Parvifolia. Eнr. Beitr. 6.97. -Willd. Sp. 2. 1078. - Pers. Syn. 2. 5o. - Smith in Rees in 1. - Вот. lieg. t. 452. - Prosv. Nom. p. 53. - Lilem. Somm. p. 27. - Makt. P. $_{\text {. }}$ - MLL. Sp. 34.

R. Burgundiaca. Roess. Ros. t. 4. - Gmes. Lad.-Als. 2. 43 1.- - Dیt. Lus. 1. 339 .

R. Remensis. Desf. Cat. I-5. - Decandolle. Fl. fr. 4 433. - Mer. Par. 191 .

Se trouve dans les montagnes, prés de Dijon (Durande). ( $V$. v. cult.)

Petit arbuste sombre, épais, d'un gris bleuâtre. Branches quelquefois glanques, droites, relevées, faibles, garuies de quelques aiguillons inégaux, épars, laibles, un peu courbés, avec des soies. Feuilles des plus gros rameaux deux fois aussi longues que celles des extrénités, qui sont tres-rapprochées. Stipules linéaires, presque nues, lrangées de grlandes verdâtres. Pétiotes velus, arıés de quelques petits aiguillons épars, droits et glanduleux. Folioles de 3 à 7 , ordinairement 5 , petites, raides, ovales, airguës, plates, simplement et finement 


\section{5}

dentées; serratures avec des glandes d'un vert foncé, d'une couleur cendrée, arec une nervore velue et des veines saillantes : la paire inférieure, quand il y a plus de trois folioles. genéralement plus petite. Fleurs solitaires, surmontées par les jeunes pousses, sans bractíes, couleur pourjere, tonjours trés-doubles. Pédoncules sans poils, mais avec quelques soies (1). Tube du calice nu on ovale. Sépales orales, nues, réfléchies, concaves et sans divisions, munies de quelques glandes, beancoup plus courtes que la corolle. Pétales étalés, excepté ceux da centre, qui sont formés par les oraires, et très-imbriqués. Styles velus, un peu saillans et adhírens par leur duret.

J'hésite peu à le distinguer du dernier, appuyé de l'autorité et des observations exactes d'Ehrhart. Il difiere certainement du gallica comme celui-ci differe du centifolia. Comme je n'ai aucune variété à citer, il est moins diflicile de fxer les caractères qui le distinguent. Je l'ai vu crôtre dans le terrain le plus ingrat comme dans le pius fertile; et dans ce cas, sans aucuue altération de part et d'autre dans son habitusle, et notamment sans prendre la moindre tendance à rece. voir les carilcteres dı gallica. Sur l'autorité de M. Decandolle, on assure qu'il a étí tronré en état sauvagre dans les montarnes des euvirons de Dijon.

"J'ai déj olser"è que le R. parvifolia est celui que nos jardiniers appeltent le petit St.-Francois. Je cultive, sous le non de Bourgogne. un petit rosier il fleurs simples, qui porte tous les curacteres de la variélé $y$ pomponia, du rosicr à cent feuilles. „

$$
\text { VIE. THBU. - VILLOSZE. }
$$

Caractères particuliers -Surculistricti; acnlei rectiusculi. Foliola ovala vel oblonga, serraturis divergeulibus. Sepala conuiventia, persistenii.ı. Discus incrassatus, fitucem clauden:

Obs. Cette tribu " de grands rapports a vec celles des rubiginosie et de-canin.e. Elle se distingue de l'une et de l'autre par el's jets dioits et vigoureux qui ne se courbent point avec

(1) Je crois avoir fuit déjà sentir la difićrence entre soyeux nu pourva de soies (setigerus). Les soies du rosier sont des poil, trèsi igides et droits commo less sisies du sanglier. (JoF FAIX.) 
grâce, exceptè dans le véritable tomentosa. Le caractère distinctif entre les villosa et les canince, se trouve dans la forme des aiguillons, lesquels sont droits dans la première, et crochus dans la seconde; et aussi dans la divergence des serratures des folioles, qui n'a pas lieu dans les canina. Je ne connais pas un seul exemple qui souffre exception. Si, comme il arrive quelquefois, les aiguillons de celte tribu sont un peu courbés, les serratures ne divergent que plus évilemmeut; lorsque celles-ci convergent, les aiguillons deviennent plus droits. Le premier élat a pour cause lia luxuriation, et le second la débilité. Le contraire arrive dans les canina: la persintance des sépales est un autre caractère qui distingue cette fantlle de celle des canince.

Les rubiginose ne peuvent être confondus avec les villosce en raison de leurs aiguillons crochus et inégaux, et de leurs feuilles glanduleuses. La rudesse du fruit et la persistance des sépales sont communes à l'une et à l'autre; le rosier villosa porte toujours des liruits hispides.

\section{ESPÈCE. - ROSA TURBINATA.}

R. Calycis tubo turlinato. Linox. Mon. 73 .

R. Francofurtiana. Hunca. Hausw. 5. 24. - Bork. Holez. 312.Gmel. Bad.-Als. 2. 405 .

R. Turbinala. $\Lambda_{1 \mathrm{~T}}$. Kew. 2. 406. - Willn. Lo. 2. rom3, - Lour. 1. 63. - JACQ. Schoenbr. 4. t. 415. - PErs. Syn. 2. 49.- JACQ Fragm. 71. t. 107 . f. 2 ? - Sмiтн. in Rees in 1. - Rau. Enum. 48. - Redout. Ros. 1. 127 . t. 48 . - Provv. Nom. r. 36. - Idem. Somm. p. 29.

R. Compunulata. EHr. Beitr. 6. 97 .

R. Francofurtensis. Roes. Ros. t. i i, - Desf. Cat. 175.

Se trouve en Allemagne, où il croit dans les vignes et les buissons. (V. v. cult.)

Arbuste de la grandeur et de l'aspect général du damascena, dont il differe en ce qu'il n'a point de soirs. Des aiguittons égaux et courts, des sépates ovales et entières, et le tube du calice turbiné, tels sont ses caractires. On ne connaît pas bien son lieu natal. Rau assure qu'il croît en Allemagne, et cite les lieux où il se trouve, tels que les hords des vignes et les buissons. 'Toutefois comment une fleur si double peut-elle se propager par ses graines, ou bien comment se rencontre-t-elle ainsi placée à moins qu'elle n'y ait passé des jardins? 
Jacquin, daus ses Fragmens, a figuré le fruit comme venant de l'état simple de ce rosier; mais il est oblong et d'un spécimen appartenant it une autre espéce.

a J'ai va cultiver ce rosier dans l'état simple à Trianon; une très-belle variété obtenue à Lille est nommée grande pivoine. Je ne vois pas le $R$. turbinata fort recherché par les pépiniéristes."

\section{כy". ESPECE. - ROSA VILLOSA.}

R. Foliolis ellipticis, obtusis ; fuctu maximo, armis rigidis, confertis horridn; sep.lis viscosis, hispidis. Lisnc. Mon. 7ł.

R. villosa Livм. Sp. pl. 7of. - Wıи. Sp. 2, 106g. - Sмлтн. Brit.

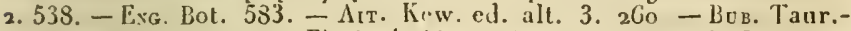

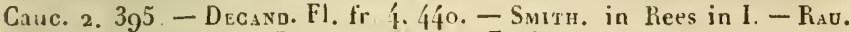
Enum. 15n.-Rrpout. Ros. t. 67. t. 22. Excl. lig. fruct. - Lawr. Ros. t. 39. - Pronv. Nom. 3. - Mlart.-Ap. - Mill. Dict. Sp. 12. - Pron. Somin. P. 29.

R. Ponifera. Herm. Diss. 16.-Bork. Halz. 3og. - Gmez. Bad.-Als. 2.

R. Gracilis. Wouds. in Act. linn. 12. 186.

Se trouve dans le nord de l'Angletcrre (Wooos); en France (DECANDOLLE); prés de Wurtzhourg ( $\mathrm{K}_{\mathrm{Au}}$ ); montagnes boisécs de la Tauride (Bubensteis). ( $V$. v. cult. et s. sp.)

Le plus grand arbuste du genre, formant quelquefois un petit arbre dont la lige est grosse comme le bras. Branches d'un aspect peu agréable, tres-glauques, sans aucune teinte rougeâtre ou à peu près, armées de quelques aiguillons forts, droits, quelques-uns en faux, et épars ou placés sous les stipules avec peu ou point de soies. Feuilles ordinairement grandes, grises, couvertes partout d'un duvet assez dense. Stipulcs étroites, aignës, finement dentées et frangées de glaniles. Pétioles glanduleux avec des aiguillons petits et courbés. Folioles environ 5, très-inégales, elliptiques, plates, rugueuses, exhalant, Iorsqu'on les froisse, une odeur. de térébenthinc, grossièrement et doublement dentées, les serratures divergeutes. Fleurs disposées par paires, blanches ou rouge-foncé. Bractées grandes, ovales, concaves, rugueuses, sauproudrées de blanc, glabres en dessus. Pédoncules trèsconrts, défendus, ainsi que les calices, par des soies raides, inégales, et des glandes visqueuses. Tube ovale, glauque. Sépales ćtroites, composées, étalées. Pétales plus longs que larges, presque en cœur, un peu crénelés à leur limbe. Disque élevé, peu épais. Styles velus, séparés, ordinaire- 
ment plus courls que le truit mor. Fruit ou pourpre ous orangé funcé, rond, cunvert de suies, et couronné par les sépales d'un brun pâle, conniventes ct visqueuses.

La diffirence entre cette plante 't la suivante ayant été mal comprise par la plupart des hotanistes, il est très-diflicile, et quelquefois inêmo impossible de se tirer dẻ leur synonymie sans avoir des échantillons authentiques.

Là défuition caractéristique de Linné (germinibus globosis aculeatis, peduuculis hispidis, eti.), par laquelle if prétend faire contraster la rigidité et l'armure du villosa avec la faiblesse de crlles du tomentosa, ne nous permet pas de douter qu'il n'ait voulu caractériser la première ; car il n'y a point de circonstance d'âye où l'on puisse dire que le fruit du tomentosa snit aiguillonné. Toutefois 11 . Woods, jugeant d'après des spécimens marqués villosa dans l'Hierbier de Linné, a considéré, malgré son caractère spécifique, comme espèce distincte celte variélé du tomentosa que j'it appelée mollis; mais le possesseur actuel de cette collection m'a assuré que le spécimen en question ne pouvait faire autorité, parce qu'il y fut placé aprés la publication de la $1^{\text {ra }}$. édition dus Species plantarum. Néanmoins cela me confirme dans l'opinion que Linté ne distinguait pas les deux plantes; du moins on ne le trouve ni dans ses ouvrages ni dans son Herbier; et Afzelius appuie encore cette opinion en nous assurant que le tomentosa seul crnit dans lés lieux indiqués par Linné comme produisant le viliosa.

Le point de différence le plus essentiel entre ces deux plantes se trouve dans le fruit, qui est couvert, dans le viltosa, d'un grand nombre de soies et mênıe d'aiguillons, tandis que le tomentosa ne peut janais être qualifié que d'hispide. Le fruit est heaucoup plus gros et charnn dans le premier que dans le second. Les leuilles du villosa sont plus grandes, plus exactement elliptiques et plus profondément dentelées. Les fleurs paraissent ordinairement par paires arec des supports ineigaux, le plus long s'inclinant élégamment a mesure que le fruit grossit. Ses jeunes pousses sont remarquablement glanduleuses, comme dans l'alba, et l'on remarque une tendince moins forte à produire des soies et des glandes sur les rameaux. La plante curieuse que $\mathbf{M}$. Woods appelle gracilis, porte des soies nombruses, entremêlées parmi les aiguillons, ayant aussi beaucoup de caractères de la tribu des centifotice. M. Sabine l'a découverte prarui do 
jeunes plantes élerécs de graines du rosier commun (vitlos $($ ) dans la pépiniére de $H$. Lee, a Hammersinith. "Co rosier, que j'ai vil cultive chez M. Noisette, tient beaucoup de l'alba."

"Le villosa de Pallas semble être plutôt une variété du rubiginosu."

Cie rosier croit dians les contrées septentrionales et tempirées de l'Europe, et dans le tord de l'Asie, intis non en abondance. "Milgré sa forte végétalion, les pépiniéristes n'ont pas trouvé qu'il pat servir de sujet pour gr'effes.

\section{ESPECE. - R. TOMENTOSA.}

R. Foliolis ovatis, acutiusculis; fructu hispido nudove. Lisor. Mon. p. 77 .

Var. a Vira. Surculis arcuatis; sepalis compositis.

R n. Iro5. HaLl. Belv.

R. Villusn. Duror. Harbk. 2. 34r. - Iluds. Angl. 219.

Var. b. Mofnich. Mell. 688. - Af\%. Tent. prim, - Mer. Par. 190. FL. dan. 1. 1458. - Desv. Journ. bot. 2. 1 17 .

R. Mollissma. Bокк. Halz. 3oz. - WiLLd. Prodr. fl. boreal. 1237. GMEL. Bad.-Als. 2. 409 .

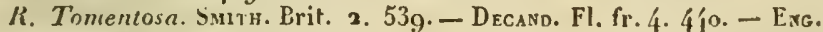

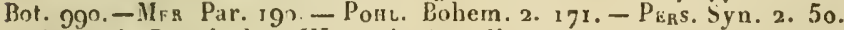
- SMith. in Fees in I. - Woons in Act. linn. 12. 197. - Redout. Kos.

2. 39. t. 17. - Prosv. Nom. p. 97-Idem. Soum. p. 3o.

R. Dubia. Wiвеz Wirth. 263.

R. Scabriuscula. ENG. Bot. t. $18 g 6 .-S_{M I T}$. in Rees in 1. - Woods. in Act. liun. 12. $19^{3}-$ Wisch Ess. geogr. 43.

R. Fetida. ba r. Suppl. 29.-Decand. Suppl. 534. - Redout. Ros. 1. x 3 r. t. 50 .

Var. b. Mlocts. Sirculis strictissimis; sepalis subsimplicibus.

R. Villosa. ViL. Delph. 355r. - Woons. 1. c. 12. I89.

R. Mollis. Eag. Lot. t. 2459 - Ммитн, in Rees in 1. - Wisch. Eis. geogr. 42.

R. H.terophilla. Woovs. I. c. г2. in5.

R. Pulchella. Woovs. in Act. linn. 12. $19^{6 .}$

R. Villosa minuta. Rav. Enum. 160 .

Var. y. Resinosa. Pumila, cæsia; foliolis angustis; floribus ruberrimis.

Se trouve dans toute l'Europe, dans les haies sauvages. En Irlande (Drosimond) : dans les bois de Ilcudon (ThORY). ( $V$. v. cult. et s. sp.) Var. y. Herb. Hooker. (Lindi.).

Arbuste te $z$ it $S$ pieds de haut, étalé, très-gris. Branches quelçuefois glauques, armées d'aiguillons droits, rarement arqués, ṫaux, épars et sans soies. Feuilles, couveries d'un du*et hianchâtre. Stipules concaves, dilatées, dentelées et fran- 
gées de glandes. Pétioles largement aiguillonnés et glanduleux. Folioles environ 5 , oblongues ou ovales, obtuses, doublement dentés, serratures diver gentes, rarement convergentes, denses et rugueuses, plıs pâles en dessous, et quelquefois glanduleuses, exhalant par la pression une odeur de térébenthine. Fleurs pręque solitaircs, rouges, en coupe, avec de courts supports. Bractées ovales, colcnneuses, plus longues cu plus courtes que les pédoncules, qui sont hispides avec des soies inégrales ou des glandes. Tube du calice ovale, nu obrond, ordinairement hispide, quelquelois glalirc. Sépales composécs, étalies, tuujours hispides en delsors. Pétales entiers, presque en cour, concaves. Disque épaissi et plat. Styles tris-velus, distincts. Fruit pourpré, rond on presque ovale, dépriné, ordinairement hispide, courontí par les sépales convergentes, mais celles-ci se détachant immédiatement après la maturité du fruit.

Si j'ai cru devoir rapporter le $R$. mollissima de Borkhausen à celte espèce, j'aurais da peut-être lui donner la priorité sur le tomentosa de S. J. Smith; mais comme cela ne peut se faire à moins d'avoir sous les yeux des échantillons authentiques, j’ai préférí lui laisser le nom plus généralement adopté.

Si l'on en excepte le canina, cette espèce est une des plus variables du genre. Et les variétés principales offrent peu de caractires pour assigner i chacune d'elles une définition palpable, telle qu'aux $R$. tomentosa et moltis de la Bolanique anglaise, et au resinosa des manuscrits de M. Lyell.

Le premier a les feuilles glabres en dessus (tomentosa 6 de Woods, et le foetida de Bosc), ou glabres des 2 côtés (tomentosa $\psi$ de Woods), ou sans glandes (tomentosi o de Woods). Le fruit est long, rond, deprimé on turbiné, bispide, glabre ou à peu près. Lès fleurs sont pâles, carnées, rougefoucé ou mouchetées comme dans la figure scabriuscula de la Botanique anglaise. Celte plante est très-commune dans le couté de Suffolk, et peut a voir embarrassé $\mathrm{Ml}$. Woods pour discerner en quoi elle diffère du tomentosa; dans le fait il y a une dissimilarité indicible dans leur aspect général, et en particulier dans les feuilles du premier, qui sont plus grandes; bien loin que la pubescence soit plus prononcée dans le tomentosa,c'esi précisiment le contraire. La plante que $\mathbf{H}$. Winch a trouvće près de Newcastle a les folioles plus aiguës que celle 
de Suffulk, qui d'ailleurs est très bien Rirutée dans la Botanique anglaise.

Le foetida du supplément de H. Bastard i la Flore de Maineet-Loire est une fiuble variété, avec des fenilles glabres en lessous; son fruit répand, dit-on, une odeur fétide lorsqu'on l'écrase. Le Reynieri rapporté ici par Woods me parait être le rubiginosa flexuosa.

La valiété \& mollis a certainement un caractère bien saillant dans son mode de croissance pour la distinguer de la première. Ses rejetons ou drageons sont très-droits et non arqués comme ceux de la première. Toutefois je ne sais si ce caractère paraîtra suffisant sans y ajouter d'autres particularités. Lès sépales non divisées sont assez constantes. Mais j'ai des spécimens que 11 . Lyell m'a procurés d'une plante du Northumberland qui produit l'un et l'autre. Elles sont un peu divisées dans le $R$. heterophylla et très-entières dans le pulchella, jui a toute l'apparence du mollis dans l'état rabougri firguré dans la Botanique anglaise.

Plusieurs spécimens du tomentosa ont des sépales parfaitement intermédiaires entre le simple et le composé. On ne doutera pas que lis distinction entre simple et presque simple soit un caractère trop vague pour établir une distinction entre les variétés d'une même espèce. J'ai examiné les spécimens du pulchella de $\mathbf{M}$. Woods sans pouvoir découvrir les crénelures des pétales ( 1 ), sur lesquelles il a établi son espèce, et l'or? ne peut les considérer comine caractère suffisant, à inoins que la proportion comparative des fleurs ne soit également admise, puisqu'il arrive que des pétales mutilés ont leur limbe plus ou moins crénelé. Les plantes de l'hibernica du jardin de 11. Lyell avaient des pétales crénelés pendant une saison, et des pétales marginés dans la suivante.

La variété ఫ resinosa est une plante fort intéressanie, et l'on peut croire qu'elle a pour type le tomentosa de Rau, de même que l'aciphylla vient dı canina. Les échantillons, pris dans l'état sauvage, sont plus petits dans toutes leurs parties, avec un feuillage très-épais, des feuilles grises. ou des fleurs d'uu rouge clair. J'ai cependant un spécimen intermédiaire d'me plante provenue de l'Irlande dans le jardin de M. Lyell, qui devient tous les ans plus robuste et moins blanchatre. Elle a été trouvée dans le uidi de l'Irlande, par M. Drummond, di-

(1) M. Norsette cultive un tomentosa i neurs blanches semi-lonbles et à pétales crénelés. 
recteur du jardin botanique de Cork, et j'en ai reçu un échan tillon par M. Hooker.

Le tomentosa et le canina se rapprochent tellement, que pour établir un caractère différentiel, j'ai proposé de prendre les aiguillons droits, les serratures divergentes, les calices hispides ainsi que les sépales, les pédoncules du premier qui contrastent avec les aiguillons crochus, les serratures convergentes et le calice glabre du second. Les sépales caduques du canina et ses feuilles rarement pubescentes peuvent aussi le distinguer. Une variété du canina à feuilles cotonneuses, que l'on trouve à Fontainebleau, a été prise pour un villosa par M. Thuillier. Le tomentosa a les sépales droites pendant la floraison. Toutefois j'ai des spécimens de Chamouni rapportés par M. Hooker qui les ont réfléchies.

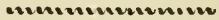 \\ $41^{\circ}$ ESPÈCE. - ROSA SPINULIFOLIA.}

R. Foliolis ovatis, acutis, spinulosis ; fructu hispido-muricato. N.

R. Germinibus ovatis pedunculisque spinosis; calycibus pinnatis, pinnis linearibus; pedunculis villosis, aculeatis; foliolis suprà glabris, infrà spinulosis. Dematra. Essai. p. 8. sp. 10.

Var. a. R. Spinulifolia dematratiana. Tнопу. Prodr. p. 115. fig. Z.

Var. $\beta$. Aculeis valdè falcatis; fructu hispido nudove. N.

R. Spinulifolia foxiana. Tnony. Prodr. p. 116. fig. 2.

R. Pseudo-rubiginosa. Lejeune. Fl. de Spa. 1. p. 229.

Se trouve près de Fribourg en Suisse (Dematra); la var. $\varepsilon_{\text {. aux envi- }}$ rons de Malmedy. ( $V$. v. cult.)

Ce rosier, que M. Thory cultive à Clamart sous Meudon, et dont il a formé son $21^{e}$. groupe sous le nom de spinosulce, a été découvert en Suisse par M. le doyen Dematra, curé de Corbière. Je ne l'ai pas vu franc et dans l'état sauvage; mais sa croissance paraît vigoureuse. Les aiguillons de la tige et des branches sont droits, forts, quoique peu épaissis à leur base, quelquefois opposés; les feuilles coinposées de 5 à 7 , ovales, aiguës, fortement et doublement dentées, serratures divergentes, d'un vert clair en dessus, plus pâles en dessous; chaque nervure couverte de petites épines ou aiguillons droits, sensibles au tact, et munis de glandes. Pétioles velus et aiguillonnés. Stipules oblongues, concaves, bifides, velues et frangẻes de glandes. Pédoncutes et calice très-hispides, avec quelques aignillons. Sépales hispides, presque simples, mais très-longues et subulées. Tube du calice ovale, 


\section{.85}

nhrond. Fleurs moyennes, d'un rouge pâle. Pélates en cneur. Styles séparés. Les fleur's et les teuilles supérieures exhatent une forte odenir de térébenthine; je n'ai pas va le fruit mno, et ne puis assurer s'il reste couronné par les sépales, mais celles-ci sont un peu réfléchies.

Lit variété $\beta$, d'aprés la figure qu'en a donnée M. Thory dans̀ son Prodrome, a les folioles plus petites et plus aiguēs, le tube du calice ovale, et les pédongules plus allongés. Sies aiguillons sont aussi plus courbés que dans l'espèce. Cette plante. qui me paraît avoir de grands rapports avec le villosa et le tomentosa, mérite d'être cultivée à cause du caractère singulier de ses folioles épineuses.

\section{$42^{\circ}$. ESPE்CE. - ROSA ALBA.}

R. Foliolis oblongis, glaucis, suprà nndiusculis, simpliciter srrratis; sepalis reflexis; fructu inermi. LINDL. Nom. p. $8 \mathrm{r}$.

K. Sativa. Dodon. Pempt. i 86.t. I.

R. Candida plenn et semi-plena. Bava. IIist. 2. Lí.

R. Damascena. FI. pl. albo. Beist. Eyst. vern. ord. 6. fol. 1 .

R. Alba. Linn. Sp j05.- Milu. Diet. n. 16.- Allion. Ped. 2. 139.

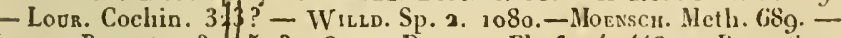
Lawr. Ros. tt. 23.25. 32. 3-. - Deennu. Fl. fr. 4. 4í8. - D'fas. Synn. 3. 49. $-\Lambda$ rr. Kew. ed. alt. 3. 267. - Gmel. Bad.-Als. 3. 427. - Fu. dan. r215. - Surmir. in hees in 1. - Rav. Enum. 9i. - Redou's. Ros. 1. 37.t. 34. 117. t. 43. - Provv. Nom. p. 81. - Ideme. Somm. p. 3o. R. Usitatissinza. Gat. Montaub. 94.

Se trouve en Piénont ( $A_{\text {LLIONI}}$ ); en Cochinchine (Lotreiro); dans les haies en Fionie (FL. dan); en France (Decandolle); dans la Hesse et en Saxe (Котн). ( $V$. v. cult et sp.)

Arbuste de 6 à 7 pieds, étalé, d'apparence grise. Branches vigoureuses, mais sans ćlégance, glauques ou vert-clair, quelquefois rougeâtres sur le côté non exposé au soleil, avec des aiguillons droits ou un peu arqués, faibles ou peu furts, inégaux, épars. Feuilles tristes (1), glauques. Stipules étroites, plates, allongées à l'ẹtrémité, presque nues, dentelées et frangées de glandes. Pétioles cotonneux, glanduleux et aiguillonnés. Fotioles de 5 à $\boldsymbol{\eta}$, grandes, rugueuses, ovales et presque rondes, obtuses ou terminées par.une petite pointe, siniplement dentées, dentelıre effilée, nues en dessus, cotonveuses et très-pâles en dessous. Fleurs grandes, nom-

(1) Dull en anglais, coulear sans éelat. 
breuses, blanches ou d'une jolie teinte carnéc, ordinairement doubles. Bractées lancícolées, cotonneuses, droites, concaves. Pédoncules munis le soies luibles èt inégales. Tube du calice oblong, III oll soyeux à la base. Sépales longues, pinnées, hispides en dehors, réfléchics et caduques. Yétales concaves, énarginés. Disque épais et aplati. Sityies relus, séparés. Fruit oblong, écarlate ou sanguin.

si le Rosa gallica est un des plus magnifiques de nos jardins, cette espèce ne lui cède guère en beauté pour ses fleurs nombreuses et doubles, lavées d'une charmante teintc rosée, ou ilublanc le plus pur. Selon $\mathbf{M}$. Winch, il est naluralise sur les bords du Tyne, quoiqu'on ne l'ait pas encore trou vé dans les campagnes de ce pays; mais il n'est pas dillicile de le rencontrer en France, en Allemagne et dans le Piémont. Il est pro. bable que celui de Loureiro est le nême.

Les feuilles rugueuses et très-glauques (I), à simple dentelure, toujuurs réfléchies; les sépales caduques ordinaircment aciculaires; les aiguillons inćgaux le séparent du tomentosab et du canina. Le tomentosa lui ressemble plus dans ses cal'actères botaniques qu'en réalité.

\section{now..... \\ $43^{\circ}$. ESPECE. - ROSA EVRATINA.}

R. Elatior. Aculeis rectis; sparsis, ramulorum suhnullis; foliulis oblongis, simpliciter se?ratis; tloribus corymboso-umbellatis; peduaculis calycibusque hispidis. N.

R. Evatina. Boss. Dict. d'agr. 1 1. p. 256. - Provs. Nom. p. 87. laem. Somm. p. 3 1. - Rosier d'Evrath. Poik. Enc. suppl. p. 21 à.

Var. ß. BKEVISP1Na.-Aculeis ramulorum brevissimis, rectis.

R. Riubrispina. Nonset're.

Se trouve en Caroline (Losc) $(V$. v. cult. et s. sp. Ilerb. Eosc.)

Arbrisseau très-fort, d'un vert obscur. Branches élalées, munies d'aiguillons presque droits, courts, quelquefois géminés, mais distans. liameaux presque entièrement dépourvus d'aiguillons et sans soies. Stipules étroites, loifurquées, frangées et velues en leurs bords. Fotiotes de 5 a 7 , d'un vert sombre en dessus, plus pâles et velues en dessous, ublongues, obtuses et simplement dentées. Pétioles velus, arisés de quelques petits aiguillons. Pédoncules très-hispi-

(1) Daus plusieurs variétés les folioles ne sont point glauques, mais d'un vert tendie. 
les ainsi que le tube du calice qui est ovale. Sépales treslongues, hispides et subulées. Bractées assez grandes, lancéolés, l'angées, rélınies 2 i 2 i claque division des pédonrules. Fleur's noinbrenses, innyennes, d'un rouge pille, ordinairement doubles.

C'est probablement la description des fleurs et la force de vérétatiou de ce rosier qui lui ant fait donner par quelques jarlinicrs le nom de $\boldsymbol{R}$. muscade rouge, quoique sous les rapports hotaniques il s'éloigne absolument da $R$. moschata. Nous le devons à M. Bosc qui l'ia apporté de la Caroline (1), :- l'a dédié à son ami M. Evrath. La surabondance des fleurs est un inconvénient, car beaucoup n'ouvrent pas ou s'épanonissent mal. Toutefois ce rosier produit un hon eflet das les jarỏius d'ayrément.

- La variété $\beta$ brevispina, a les rameaux couverts de petit. aiguillons courts et presque droits; ses folioles sont un pen lancéolées, les dentelures plus fortes et plus aiguës. Je l'ai observé chéz M. Noisette et an jardin du Luxemboures. Le nom de rubrispina ne saurait lui convenir, c'est celui d'une espèce de H. Bosc, laquelle, selon toute apparence, est le nitida le M. Lindley."

\section{$44^{\circ}$. ESPËCE. - ROSA HIBERRIICA.}

R. Aculeis inaqualibus, minoribus setiformibus; foliclis ovatis, acutis, nudiusculis, simpliciter serratis. Linol. Mon. p. 82.

R. Hibernica. Eva. Bot. t. $2196 .-\Lambda$ ï. Kew. ed. alt. 3, 26r. Sмiтн. in Rees in 1. - Woops. in $\Lambda$ ct. liun. 12.222 - l'rovv. Somm. P. 3 I.

Se tronve en Jrlande ( $T$ Fapletor ). ( $V$. v. cult. et $s . s p$. in llerb. Banks, Lоoкek, Suitu, ef(s)

Arbuste épais, de 3 à quatre pieds. Branches droites, d'un brun rougeâtre, avec des aiguillons égaux et droits sans soies. Rameaux étendus, aves des aiguillons inégaux, faibles. quelquefois très-petits. Riejets presque soyeix, couverts entièrement d'aiguillons plus longrs mais incigaux. Jont quelques-urs en hamecon. Feuilles comme celles du spinosissima sanguisorbafolin, mais plus grandes et plus aiguës. Folioles généralement 5, velues en dessous, surtont à la

(1) Po1r. Enc. suppl. M. Bosc l'a trouvé chez M, Ératu, el il ignore sou pays nafal. Bict. d’agr., z*a cil, p. $2 ; 9$. 
nervure principale, simplement dentées. Fleurs solitaires, presque tonjours sans bractées. Pédoncules ronds ou cylindriques. Tube du calice à sépales nues, celles-ci composées et réfléchies après la floraison. Pétales concaves, émarginés. Disque aplati, saillant. Fruit d'un rouge sombre et ioncé, couronné par les sépales.

Il est plus difficile d'assigner une place convenable à cette espèce qu'i aucune autre du genre. Son aspect dans l'état laible et mincur est celui du spinosissima; plus vigoureux il ressemble au canina; et s'il est trés-robuste, au tomentosa molLis; il a plus des caractères de la tribu oú je l'ai placé que des autres, et il pourrait être cunsidéré comme un chaînon du tomentosa au canina, s'il n'y avait entre ces deux tribus celle des rubiginosa. M. Woods, avec sa prétention ordinaire, lui donne comme caractère le plus important, le mélange de petits aiguillons droits sur les branches et rejetons. Il est vrai qu'il a ce caractère commun avec le rubiginosa. mais le défiut absolu de glandes, ses dentelures simples et la forme de son l'ruit suffraicnt pour éviter toute confusion.

Si ce rôsier n'est pas très-intéressant, il a paru digne d'être recherché par $\mathbf{H}$. Templeton qui l'a découvert, attendu qu'il Ini a valu 50 guinées qui lui avaient été promises par les patrons ce la botanique de Dublin, pour la découverte d'une nouvelle. plante iudigine en Irlande. Les environs de Belfast sont le seul endroit du monde oì on l'ait encore trouvé.

\section{VHĭ". TRIBU. - RUBIGINOSA.}

Aculei inæquales, nunc setiformes, rarò (an unquàm) nulli. Foliola ovala, v. oblonga. glandulusa, serraturis divergentibus. Sepala persistentia. Discus incrassatus. Surculi allcuati. - Obs. Les glandes nombrenses qui couvrent la surface inférieure des fenilles dans cette tribu snflisent assez, ie crois, pour la distinguer a'aver les autres. Quelquefois le $R$. tomentosa a des leuilles glanduleuses ; mais la forme, l'inégalité et la couleur roure des fruits des rubiginos cétablissent toujours une assez grande différence entre les deux familles. 


\section{ESHECE. - ROSA LUTEA.}

R. Aculeis rectis; foliolis planis, concavis; calycibus subinermilsts, integris. Lind. Mon. p. 84 .

R. Lutea. Dodon. Pempt. 187.- Bava. Hist. 2. 47.

R. Lutea simplex. BaUn. Pin. 4S3. - Berss. Eyst. vern. ord. 6. fol. 5.

R. Eglanleria. I ins. Sp. 203.-Wiebl. Wort. 263. - Roth. Germ. 1. $217-2.553$. - Decand. Fl. fr. 4. 437.-Pens. Syn. 2. 47.-Mfr. Par. 1Sg. - lienout. Ros. 1. ô. t. 23.

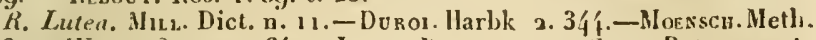
688. - WilLd. Sp. 2. 1064. - LaWr. Kos. t. 12. - Curt. Bot. mag. t. 363. - Aıt. Kew. 3. 258. - Gmer. Bad.-Als. 2. 463. - S мitr. in Réz in 1. - Rav. Enum. 15\%.- Pronv. Somm.

R. Fetida. Herm. Diss. 18. - Allion. Ped. 2. 138 :

R. Chlorophylla. Enr. Beitr. 260.

11. Cerea. Roessig. hos. t. 2.

Var. $\beta$. Punicea. Floribus bicoloribus.

R. Sylpestris custriaca. Flore puniceo. Новт. Angl. 66.

R. Punicea. Mitl. Dict. n. 12. - Dorol Harbk. 3. 3ín. - Rioess. Ros. t. 5 .

R. Cinnaniomea. Rотн. Germ. т. 21 \%. et 2. 554 .

R. Lutea bicolor. Jace. Vind. 1. t. 1.-Lawr. hos, t. 6-Sinus. Bot. mag. t. ro77.-A1т. Kew. ed. alt. 3. 258. - Sm1'11. in fiees in 1.

h. Eglanteria punica. Pienout. Ros, 1. 71. t. 24.

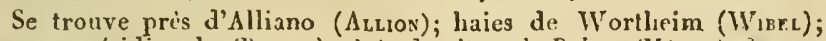
France méridionale (KequIN); à Andresis, prés Poissy (Mérat); la var. Ben Autriche (Jaceurs). (V. v. cult. et s. sj.)

Arbuste pen ramenx, de quatre pieds de hauteur (le même en a de sept à huit) dans l'état sauvage. Branches un peu droites, luisantes, d'un brun foncé, défendues par des a $i$ guillons pâles, droits, presque égaux, épais et sans soies; l'armure des rejetons est plus épaisse. Feuilles quelquefiis luisantes et visqueuses (surtont au printemps). Stipules étroites, dilatées et divariquées à leur extrémité avec dentclures fines, frangées de glandes, un peu pulsescentes ou point du tout. Pétioles nus ou cotonnenx, rarement glanduleux. Fotioles, de cinq à sept, elliptiques ou ovales, un peu pointues, en cuillère, simplement ou doublement dentelées, noires en dessus, plus ou moins velues et glinduleuses en descous. Fleurs grandes, jaune-foncé (jonquille), en godets, solitaires. l'oint de bractées. Pédoncules et tube du calice inermes; ce dernier est ovale. Sćpates ovales, pointues, un peu divisécs, soyeuses, et comme aiguilionnées en dehors. Pétales presqu'en cœur. Hisque épaissi. Styles rolus, séparés. Fruil rond (dans sa maturité). 
C'est bien mal à propos que quelques botanistes ont confonducette espice a recle sulfurca(1) comme l'observe S. Ja nes Smith; il n'existe entre ces deux rosiers que la similarité de couleur. Le sulfurea est allié anx sabinea, tutescens, etc., et celui-ci se rapproche du rubiginosa. On sait que Linné ne les distingue point d'abord et les réunit sous le nom d'eglantevia. M. de Theìs nous dit que ce nom devrait être écrit $a i-$ glanteria, étant dérivé de aig du celtique ac, qui signifie pointu. Les bolanistes français s'accordent pour considérer le tutea comme le vérilable églanteria, de Linné; et, en conservant ce dernier nom, ils ont rejeté celui de Miller. En tant que l'on peut accorder d'autorité à l'herbier de Linné, il est en leur faveur; quant à moi je m'en rapporte à Willdenow et autres qui ont conservé la dénomination de lutea, de préférence à une autre qui u'est nullement expressive, ni généralement adoptie; et si nous pouvons juger d'après ce que dit linné dans la première édition de son Species, il avait d'abord en vue le rubiginosa.

On le reconnaît au premier coup d'ocil à ses branches qui ne portent des feuilles qu'à leur extrémité, ordinairement plusieurs aiguillors sous les stipules, et les folioles concaves ou en cuillère. Les seuls échantillons spontanés que j'ai vus, avaient été recueillis près d'Avignon par M. Requin : ils appartiennent a l'herbier de H. Hooker.

- Mon $R$. Lutea pallida vient du grand potager de Versailles; il a éci oblenu de la graine du lutea bicolor. Ses rameaux sont trìs-épineux, et ses fleurs couleur de soúre. Le rosier mal nommé par ll. Vibert, spinosissima a fleurs jaunes, n'est lui-même qu'unè variété hybride dı lutea dont les folioles sont plus petites et les branches plus fétillées .

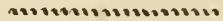 \\ 46. ESPECE-ROSA RUBIGINOSA.}

li. Aculeis aduncis. Foliolis rugosis, opacis; calycibus pedunculisque lispidis. Jinde. Mon. p. 86.

Var. a. Vulgaris. Aculeis fortibus, valdè iniequalibus; stylis villosis; fruclibus ovatis, v. oblongis.

lí Sylvestris orlorata. Dodon. Pempt. 186. Ll. 2.

(1. Les grems du monde et les jurdiniers peu inslruits ne manyuent junats de confondre ces denx especes, en prenant le sulphurea pour une variété double du luea. 
R. S'yestris, filits olluratis. lisbm. Pin. 483

R. Foliis oiloritis, ete. BaUn. llist. 2 1.

Ii. Sylivestris odurre. GER, 108\%. 1.

R. - R. succisa. $4 \mathfrak{i}^{3}$.

li. Esclanteria. Mill. Dict. n. 4.-Dunor. Harbk. 2. 336. - IJuss.

Augl. 2 IS. - Afz. Tent. r. - IVuods. in Aet. limn. 12. 206.

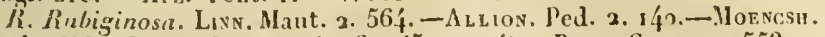
Meth. 688.-LAwr. Ros. (t. 41.61.65.72. 7f́).-Rotu. Germ. 2. 555.Willd. Sp. 2. 1073. - Suitu. Brit. 2. 54o. - Scukunr. Bot. liandb. t.

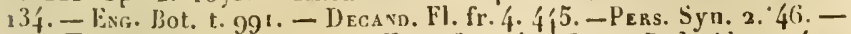

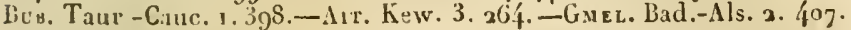

- Smitur. in Rees in I.

R. Suarifolin. Litatroot. Scot. 1. 26. Scot. 1. 262. - Fl. dan. t. 870.

11. Pseuilo-rubingiansa. Lejevne. FI. dea env. de Spa. M. Desvaux.

R. Rubiginosa vulgaris. Rav. Enum. 130. -Glabra. Lsu. I. c. 137.

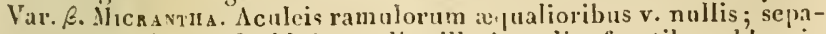
lis ante maturitatem deciduis; stylis villosiusculis; fructibus oblongis, v. obovatis.

R. ()Noratissima. Scap. Carn. т. 35 i.

Ii. $\left({ }^{* *}\right)$. CRantz. Stirp. austr. 1. 82 .

R. Eglunteria rubial. Liosss. hos. t. 10.

R. liubiginosn. JACQ. Austr. 1. 3 1. t. 5o.

li. Cruntzii. Schultes. Olss. g'́

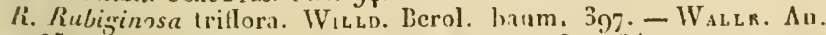
hot. 65. - Rad. Enum. 134. - Redout. lios. 1. 93. t. 3 \%

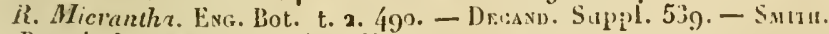
in Rees in 1. - Woops. in $\Lambda$ ct. liun. 12. 209.

R. Eiglanteria americana. $\Lambda$ xorews's hose's. c. fig.

R. Sunveolens. Puksa. An. scpt. n. 11.- Sivitu. in Rees in I.

1R. Nemorosa. Le Jeune. Spi, 2. 311. ex lienout.

Ii. Rubiginsa resinosa. IValln. An. I, it. 65. - Irlem parvifolia. Fiu. Enum. 135.

R. Rubiyinosa nemoralis. Renout. Ros. 2. 23. t. 10.

Var. $\gamma$ Unbelata. Inflorescentia ramo!is aculeatissimis; fructions elongatis.

$R$. Umbellata. Leens. Herb. I g. - $\Lambda$ dD. 2 S6.—GMel. Had. - $\Lambda$ ls. 2. 435.

-D. C. Suppl. fl. fr. 332. - Rau. Enum. 1 ́o.

R. Sempervirens. Roth. Germ. 1. 218. 2.536.

R. Tenuiglandulosa. Mer. Par. 189 .

R. Eslanleria cyricosn. Woous. in Act. linn. I. c.

Var. \&. Grandiflona? Foliis undiusculis; lloribus maximis; fruchu parpureo.

R. Grandiflora. IVALLn. An. bot. 66 .

Var. E Frfxuos.s. Ramis valde flexuosis; foliulis sulurbiculatis; bracteis deciduis; floribus subsolilaribus; stylis impulibus.

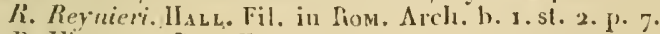

li. Irexuost. Iiav. Enum, 127 .

K. Mreuritaza. Decavn. Sinpli. 53\%.

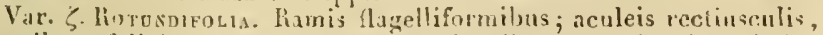
feurbus; foliolis subrotundis, dupliominorihus; calycis tubo subytoJoso, sla!mo.

li. Lubiginssa rulundifolia. lisu. Lnun. 136, 
Var n. Sertm. Fanis debilibns, flexuosis; foliolis utrinquè acutis; floribus subsolitariis; fructibus glaberrimis, sepalorum laciniis angustissimis.

J. Helvetica. II tu. Fil. in Kom. atch. b. r. st. 2. p. 6. MSS.

I. Myrtifolia. HAst. Fil.

'i. Canina f. DC. Fl. fr. ed. 3. 3־16. ex DC.

Ji. Sacpium. Tн. Par. 252. - Nr.. Par. 192. - Decand. Suppl. 538.

Ni. Agrestis. $\mathrm{S}_{\mathrm{Av}}$. Pis. 1. $475 .-\mathrm{N}_{\Delta \mathrm{T}}$. Mend. t. 27. ex DC.

R. Bisinata. MER. Par. 190.

Ii. Nucrocarpa. Ih. Fide Desvat.

R. Stipularis. Id. Yide Drsv.

Var. P. INodora. Aculeis valdè aduncis, subrertualibus; foliolis minùs glandulosis; sepalis ante maturitatem deciduis.

R. l'illosa. Pal L. Piass. 63.

R. Inorlora. A gak th. Novit. 0 .

fi. Dumetorum. Exg. Bot. t. 25/9. - Sм1тн. in Rees in 1.

li. Bomeri.Woods. in $\Lambda$ ct. Jinn. 12. 210.

Var. 4. Tendig and losa. Aculcis aduncis; minoribus, rectiusculis; foliolis villosis; jeduticulis brevissinis; frucibus subrotundis, glabris.

R. Tenuiglaniulosa. Mrk. Par. ed 2. p. 363.

Var. i. Parvifurı. Pumila; ramis setigeris; foliolis subrotandis.

Se trouve dans les halliers des montagnes de la Tauride. (V.s. sp. llerb. Cel. Lameest.)

Singulière variété naine du rubiginosa trourée par Pallas dans les montagues de la Tauride. Branches nues, légèrement soyeuses, et ses feuilles petites et rondes comme celles du micracantha. Lride.

Sc iroure en alundince dans toute l'Europe; au Caucase (Boв.) : $r . n$. en Angleterre, mais rarement en Allemagne (Rотн); en Franre (Drcasnolle); var. n. pris Wurtzbourg ( $\mathrm{R}_{A \mathrm{U}}$ ); var $\mathrm{n}$ en Franee (DEC); var. n. près W Vurłzbourg (hav); var. d. en Angleterre, Pussie (PALlas); Sucde (Agardi); var. n elvirons de Paris (MÉRAT).( $V$. v. cult. et s. sp)

Arbuste très-branchu, de trois à six pieds de haut, plus compacte dans son port que le canina; branches d'u menu clair, flexibles, armées de forts aiguillons, nombreux, en hamecon, inćgilux et épars; les rejetons sont garnis de plus petits aiguillons et de quelques glandes; fcuillage triste. verl, odorant, convert en dessous de glandes rousses; stiputes dilatées, dentelées, velucs en dessous; pétioles garnis de quelques aiguilluns forts ct arqués; folioles de cinq à septr arrondies et pointues, doublement dentelées, quelque peu concaves, ordinairement nues en dessus, relues, trüs-pâles et rugrueuses endessuus; flcurs solitaires ou deux i trois réunies, concaves, d’une cuuleur pâle; bractées pâles, lancéolées, aignës, concaves. lingerenent velues et glandulenses; pédoncules il calice hispides arec de failıles soies; tubc 
nvale; sépales réfléchies, pinnées; pétales presqu'en cour; disque peu épaissi; ovaires trente à quarante; siyles velus, sipilrés. Fruit rouge-orangé, presque rond, oblong ou obovile, hispide ou glabre, couronné par lessépales ascendantes.

Je me réserve à l'article suivant d'expliquer pourquoi je ne saurais être del'avis de $\mathbf{M}$. Woods, qui llonne au rubiginosa l: nom d'eglanteria, quoique ce ne soit pas celui de Linné. Si ce nom doit ĉtre conservé, ce n'est point assurément à l'espéce dont il est ici question

L'apparence la plus ordinaire de cet arbuste est un buisson épais et tris-branchu, couvert de fleurs réunies au nombre de trois, d'un rouge pâle, d'un truit écarlate, soyeux ou d'un vert clair. Les leuilles ne sont pas luisantes, mais elles exhalent assez généralement une fort agréable odeur. Tous ces caractires sont pourtant sujets à de grandes variations, ce qui a conduit à former une inultitude de prétendues espèces. Plusieurs ont été abandonnées par leurs auteurs, et celles qui restent peuvent être réduites à sept groupes naturels auxquels j'ai attaché les meilleurs caractères que j'ii pu trouver.

La var. $\beta$. micrantha fut d'aborl proposée comnie espéec par. S. James Sinith dans le Bot. angl. et adoptée depuis par M. Woods, qui essaya de le caractériser par ses fruits longs et l'égalité de ses aiguillous ; mais ces varactères sont peu constans et l'on peut souvent los remarquer sur lo véritable rubiginosa. On le voit souvent dans le sud de l'Angleterre avec de très-petites flcurs; mais M. Lyell, qui a journellement l'occasion d: l'observer, tle peut le distinguer essentiellement du sweet briar ordinaire, qui est l'eglunteria rubiginosa. L'odeur des feuilles varie dans l'une et l'autre. Il y a cependant quelques particularités qui, sans être d'une grande importance, aideront à le distingucr aveo assez de cerlitude: souvent il proluit de longs rejetons trésépars et sans armure, ce quel'on voit rarement dans le rubiginosa. Ses styles sent souvent sans pubescence, et ses sépales se détachent ivant la maturité du fruil; il paraît être le rubiginosa biflora les botanistes allemands, et la variété nemoratis de Redouté. La fizmre de Jacquin, dans la Flora austriaca, parait s'y accorder; mais lo fruit détaché scmble ître plus rond que je ne l'ai jamais vu. Crantz décrit son R. ${ }^{* *}$ dans ses stirpes austriace arec des sépales entieres, et à ce!a près je n'apercois aucune différence avec le typc.

On trourait dans l'école de l'lianon un rubiginosa cana 
elensis, qui est bien celui dont il est ici question. L'eglanteria americana (rubiginosa) Rosu suaveolens de Pursh est admis par les botanistes amciricains, comme une espèce impostée et naturalisée aujourd'hui dans plusieurs provinces des États-Unis. J'ai examiné les spécimens dont S. J. Smith a liré ses délails dans l'Encyclopédie de Rees; ils avaient été envoyés de l'ensylvanie par M. Muhlemberg, et j'ai rn quils ne différaient en rien des plantes d'Europe. Les folioles ne sont pas moins rondes que celles de ce pays. Pursh n'avait pas d'échantillons, c'est pourquoi ce qu'il dit Ju calice non divisé a élé tiré vraisemblablement de la misérable figure d'Audrews, à laquelle on ne peut avoir aucune confiance.

L'umbelluta est très-commun dans les jardins, avec des fleurs semi-doubles. Son aspect est celui du caucasea: J'ai rompte jusqu'i 40 fleurs sur une branche, et tontes produisirent du fruit. Sa maniere de croître plus vigoureuse, et les ramifications de son inflorescence couvèrtes de soies el d'aižuillons droits, serrés et répandus également sur les sépales et Je tube du calice, sulihsent pour le faire reconnaître.

Ma variétésuivante $n^{\circ} . \delta$, grandiflora de Wallrave est raplportée ici sans aucune hésilation. Sa description (de Wallr.) répond assez bien à la variéte $\mathrm{D}$; maisil dit que le fruit est de mum atro-purpureais, et les fleurs d'ulı rouge pourpré. De Ilus, Ja grande din:ension de celui-ci et ses feuilles très-rertes ct presque sans luret ne sont pas le carartère du Rosa rubiginosainodora.

"M. Noiselte cultive dans ses pépinières de Paris un rósier que je crois bien être celui-ci, augmenté dans toutes ses partics par la richesse du sol. n

borsque je decus de M. Ilooker, quila tenait de Seez, lavariitéa, je ne fis ancun doute qu'elle ue devînt une nouvelle espice raractériséc yal les rameaux en zig-zagg, laibles, ronds, tris-allongés, et les styles parfaitement glahres. Cette derniere particularite n'est cependan! pas rare dans le micrantha, qui a toujours moius de pubescence sur cette partie que. l'églantier ordinaire. Des obserrations plus suiries nous ort ronvaincu de l'insuffisance dn caractère de la forme des fenilles et des ranifications, qui peut être due à des circonsances accidentelles. I.a description du R. montana de 1)candulle, dens le supplément de la Flore francaise, est cntièrement applicabie a notre variété, spécialement par sos aiguilluas lides, ijus, druits, assez grếles. Oraire oroîle. 


\section{ijj}

presque spliérience, un peu liérissé, surtout à sa base. Le meontence de Villars doii difërer de beaucoup comme espiece. ciar ect atuteur le reprisente comme ayant les styles columnteres. (Voy. R. arvensis.)

Je ne cennais la variété ¿́ rolundifolia que par la description de linu. Il dit que ce rosier s'éleve à 2 ou 3 pieds avee moins de branches que les autres. Les aiguillons des rameaux sont giminés on ojposés, grêles et assez droits. Folioles arrondies, à peine pluslongues que les aiguillons. Tube du ealice glabre et arrondi. Sépales divisées, glandulaires. Fleur's solitilires, pelites, ruse-foncé.

Nota. De toutes les variétés cultivées que je connais, celle que l'on nomme la petite hessoise a plus de rapporls avec la plante de Ran, mais les leuilles ne sont pas orbiculares. J'en possède d'antres it folioles arrondies, mais qui s'éloignent de cette variété $\zeta$ pour les autres caractères.

Je désire bien qu'il puisse se tronver un caractère constant bien pronongé pour le R. sepium de M. Thuillier. C'est li plante que M. Woods veut désigner sous le nom d'eglanteria, comme ayant été apportée dı midi de la France par AI. Hooker. Il l'a trourée en grand nombre dins deg lieux arides, sur le bord des chemins. C'est à tous égards une plante plus petite, aree des folioles d'un vert foncé, presque toujours aiguës à chaque extrémité, des aiguillons grêles et des hranchesen zig-zag; le fruit est parfaitement glabre aussi-bien que les pédoncules, et les divisions des sépales sont en nombre et en dimensions peu ordinaires. Mais malheureusement, dins un échantillon des environs de Nîmes, la transition de celuici au rubiginosa vulgaris est si complète, qu'il est impossible de déterminer auquel des cleux il ressemble le plus. Quelques folioles sont rondes et d'autres aiguës; pourtant il est surti de la même souche dont les apparences sont si dissemblables. M. Desvaux est mon autorité pour les traits synonymes de Hírat.

"Nota. Certainement le sepium de Thuillier, qui a, selon Mérat, 8 à 10 pieds de haut, et que les pépiniéristes choisissent pour grefler à une grande élévation, ne peut être celui do 11. Hooker, décrit par Lindley. Je possède des variétés cultivées qui lui ressemblent davantage."

Le $R$. Borreri de Woods, qui parait être le même que le $\boldsymbol{R}$. inodora des Novitiæ d'Agarudh, m'it donné encore plus de peine que les indéterminables variélés du canina. Il y a 
quelque embarras i placer quelques variétís entre ce dernier et le rubiginosa, et je pense que l'on peut également rapporter celui-ci (incdora) à l'un et à l'autre comme chaînon intermédiaire. Il n'est pais rare dans le voisinage de Halesworth, avec de plus petites feuilles qu'ì l'ordinaire, mais visiblement nuancées de rouge vurs le limbe. Son mode de croissance et ses aiguillons sont semblables à ceux du rubiginosa; mais les sépales sont caduques et les folioles souvent sans glandes. Quelquefois les serratur'es divergent, d'autres fois elles dirigent leurs pointes vers les extrémités des folioles. M. Lyell a un $R$. Borreri de $\mathrm{M}$. Borrer, qui végète à côté d'un $R$. micrantha, et la différence entre eux est presque nulle. C'est à la persuasion de $\mathbf{M}$. Lyell que je l'ai placé ici, car je croyais fermement qu'il se rapportait davantage avec le canina, var. A.

Le villosa de Pallas répond précisément à celui-ci, et l'inodora d'Agarudh ne paraîl en différer que parses l'ruits d'une couleur pourpre.

Nota. Je cultive le $R$. inodora que j'ai reçu de M. Sabine sous le nom de Borreri. Ses pétioleset folioles sont velus, mais surtout les pédoncules et les sépales. Il végrète vigoureusement, et a fleuri en 1825 .

La variété $\theta$ tenuiglandulosa n'était pas connue de $\mathbf{M}$. Lindley lorsqu'il publia sa monographie; elle se tronve dans la $2^{e}$. édition de la Flore parisienne de Hérat; et, d'après l'inspection d'exemplaires que je liens de M. Barre, botaniste fort zélé, je crois qu'elle mérite la place que je Jui donne ici. Si elle est le tenuiglandulosa de Mérat, on ne peut la confondre avec la variété $\chi$ intervelluta, car les fruits sont presque orbiculaires et non pas allongés comme dans la variété de Lindley.

\section{7". ESPÈCE. - ROSA PULVERULENTA.}

R. Ramulis glandulosis; foliis utrinquè pruinosis, superioribus subverticillatis. Linde. Mon. $\}^{3} \cdot 9^{3}$.

R. Pulverulenta. Bub. Taur.-Cauc. 1. 399. - Poir. Suppl. encycl. l. c. - Pronv. Somm. p. 34 .

Se trouve sur les collines près le pic de Mazana, au Caucase. (Boherstein.) ( $\boldsymbol{V}$. v. cult.) (après la chute des flcurs) Hort. Sabine. LINDLEX.

Arlouste raide et peu élevé: Aiguillons assez droits, forts, arec des rameaux entremêlés de soies nombreuses, courtes, 
inégales, avee une glande de couleur grise. Feuilles assez velues. Stipules étroites, déployées, glanduleuses, in peu ondulées. Pétioles aiguillonnés, glanduleux. Folioles de 5 à 7 , ovales, pointues, mamelounées et comme couvertes de glandes gélatitornes; leur odeur huileuse assez senblable a celle du rubiginosa (suigeneris). Fleurs solitaires, rougepâle, presque sessiles, involucrées ou entourées de quatre petites folioles horizontiles. Bractées roses. Pétoncules légèrement pubeseens. Tube da calice nu, arrondi. Sépales étalées, foliacées, a vec les divisions très-nombreuses, étroites et droites. Fruit ovale, glabre, rouge - clair, couronué par les sépales conniventes et glanduleuses. Disque presque oblitéré en bouche ronde. Styles très-velıs. Pédoncules sans poils. MS. de M. Lyell.

Plante très-remarquable, ılont je suis redevable à $\mathbf{M}$. Lyell. Elle a été introduite par M. Joddiges, sous le nom de $\boldsymbol{R}$. procox. Elle est anssi distincte du rubiginosa que de tout autre. Les feuilles florales rapprocliées qui enveloppent les fleurs, couvertes de glaules grises sur la sirface supérieure, sa petitesse et son hibitude rabougrie, la lont aisément dislinguer.

Elle est native des montagnes subalpines du Caucase, où elle fut reconnue par Buberstein.

Après le $R$. pulverulenta, M. Lindley décrit l'espèce sui. vante d'après Buberstein.

\section{ROSA CUSPIDATA.}

R. Sepalis hispidis in cuspide lineari, lanceolito, serrato, ipsis longiore productis. Bub. Taur.-Cauc. 1. 39 6.-Poir. Enc. bot. suppl.-Se trouve prés de Kisljar. Fl, en juin. Вов.

\section{ESPĖCE. - ROSA GLUTINOSA.}

R. Ramulis pilosis; foliolis incanis, suborbiculatis, viscosis. Livoz. Mon. p. 95. - Provr. Samm. p. 35.

R. Pınica, alpina. Pimpinella exact: foliis sparsis spinis incurvis, requatè purpurer. Cupas. Pampli. irl. 6r. t. ז. ex Smith.

$R$. Cretica montana. Foliis subrotundis, gtandulosis ct villosis. Tourn. Col. 43.

R. Glutinosa. Sмiтн. Prodr. Fl. grac. 1. 3 亿́.

R. Rubiginosa cretica. Rèdout. hos. 1.93.135. 1. 47. 


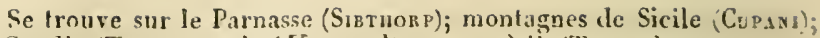
à Candie (Toenaetont). ( $\boldsymbol{V}$. v. cult. et s. sp.) Ii. ('Tounn.).

Tige basse et cn huisson. Branehes fortes et nombreuscs (Smin). Rameaux le la grosseur d'une plume d'vie, sans duvet, armés d'aiguillons lorts, serrés, inégaux et aryués; les plus jeunes cotonneux, avec des aiguillons plus petits, plus grêles et aggrégés sous les stipules. Fcuilles d'un aspect blanchâtre. Stipules fort dilitées à leur extrénité, concaves, sans glandes, excepté en leur bord qui est presque enticr. Pélioles, avec quelques petits aiguilions et des glandes. Fotioles de 5 à 7 , plates, arrondies, petites, serratures grossières, presque simples, et quelques glandes en dessous, gianduleuses et visqueuses des deux côtés. Fleurs petites, rougeJîle, solitaires sur des pédoncules courts, snjenx et grlutineux ( Бмитн). Frait sans étranglewent, obrund, écarlate, couvert en entier, comme les pédoncules, de petits aiguillons raides, couronné par les sépales grisâtres, conniventes et presque simples.

Je m'en rapporte a S. J. Smith, pour le synonyme de Cupani. Je nai janais vu de son lanpluytun que la copic de la '2. 6ı. Celui-ci șe rapproche beaucoup du rubiginosa, mais il en diffé e par ses feuilles, quasi saupoudrees de glandes grisîtres, etses rameaux pubescens, caracteres assez importans. II paraît par la figure de kedouté, oì il est moins exact que de contume, qu'on le cultive en France; mais il n'est pas encore parviulu jusqu'a nous.

"Nota. Il est assez surprenant, en effet, qu un rosier cultivé iri lepuis nombre d'années ne soit pas encore dans les collections anglaises. Les feuilles ne sont pas aussi grisâtres que dans l'etat sauvage, et la culture l'élève à environ 2 a 5 picds. Je persiste à croire qu'il ue peut être confondu avec le rubiginoste. Toutc la plante, en hiver, est d'un ronge foncé."

\section{$49^{c}$. ESPECL. - KOSA HONTEZUMZE.}

f. Pamis incrmibus. Linde. Mon. p. 1,6.-Prosv. Som. p. 35.

R. Montezumce. Hume. et Bomr. Nov. gen. et sp. tom. 3. med. - Fi:Dovt. Pios. I.ss. t. 16.

Se trouve au sonmet des moutagnes de Mlexico, latitude uord de 19 degrés, à plus de $3^{300}$ picds d'éćvation, notamment a la cime du Mnnte-Venloso, prís Saint-Pierre. (Ilumbold ét bompand.) ( $V$.v. cult. in hort. Eoursaut. Parisiis.) 
Sans armure. Branches glabres. Sliputes frangées de glandes. Pétioles cotonneux, armés de plusieurs petits aiguillons. Folioles 5 , uvales aiguës, nues des deux côtés, vertfoncé en dessus, jlus pâles en dessous. Fitcurs rouges pâles, solitaires, sans bractées, odorantes. Pédoncules nus, ailsi que le tube calicinal, qui est elliptique. Sépales composies, dilatées à l'extrémité. (Redouté, loc. eit.)

Ia description de cet intéressaut arbuste est si incomplète dans l'ouvrage de Redouté, qu'il est impossible de déternine. avec certitude dans quelle division il doit être place. Lat figure a été, selon toute apparence, dessinée d'après un spécimen desséché, et ressemble beaucoup au rubiginosa. Cependant les feuilles sont décrites comme nues des deux côtés. Si c'est réellement une espèce inerme, elle sera facile à caractériser; mais si, comme je le crois, on n'a figuré qu'une branche inerme, et si elle n'appartieut pas à la présente division, elle doit être renvoyée à la suivante, alors je ne conçis pas comment on la distinguera du camina. On dit que les pétioles sont aiguillonnés, et je n'ai pas encore vu une sersle. espèce dépolirvue d'aiguillons sur les branches, sans qu'ils ne se trouvent sur les autres parties de la plante.

Elle a été trouvée sur la chaîne des montagnes porphyritiques qui terminent au nord la vallée de Mexico, à une élévation de 1400 toises sur le sommet de Cerro-Ventoso, près la ville de S.-Pedro. Le thermomètre de M. May y était à 10 ou 11 degrés, échelle de Réaumur.

Nota. "M. Joffrin observe avec raison que M. Lindley aurait do spécifier si c'est au-dessus ou au-dessous de 0 ; mais je pense que c'est au-dessus, car les feuilles ne résisteraient pas à 11 degrés de congélation. "

\section{IX'. TRIBU. - C.ANIN EE.}

Aculei æquales; adunci ; foliola ovati, eglandulosa, serraturis convergentibus; sepala decidua. Discus incrassatus, faucem claudens. Surculi majorum armati.

Obs. La séparation et la divergence des styles empêche que l'on ue confonde cette tribu avec la suivante. Les caracteres essentiels qui la distinguent des précédentes ont été expliqués ci-dessus. M. Sabine possẻde un rosa canina hispide dans 
Jes rameaux; mais c'est une exception unique, qui ne peut diminuer l'importance des caractères que j'ai assignés à celle division.

\section{0 ${ }^{\circ}$ ESPËCE. - ROSA CAUCASEA.}

h Foliolis mollibus, oratis; ovariis 5o-6o. Lindi. Mon. p.97--PRonv. Somm. p. 36.

R. Caucusica. Palr. Ross. 62.-Bub. Taur.-Cauc. 1. 400.-A1t. Kew. ed. all. 5. 266. - Sнгтн. in Rees in 1.

Se trouve en Sibérie. (STEven). ( $V$. v. cult.)

Cette plante a une si grande ressemblance, à bien des égards, arec celle qui suit, que je doute fort qu'elles soient différentes. Celle ci se distingue ordinairement par une habitude très-vigoureuse, ses ficuilles larges et molles, ses fleurs en bouquet, ses fruits trés-gros et leur pulpe molle. Le canina, à la vérité, possède quelquefois une grande partie de ces caractères; mais les feuilles ne sont pas douces; et, au contraire, leur pubescence est rude. Le caractère le plus constant de cette espece semble etre le nombre extraordinaire de ses ovaires, lequel, dans la fleur centrale, n'est pas moindre de 50 et fréquemment près de 6o, tandis que le canina n'en contient pas plus de 25. Pallas dit n'avoir eu sous les yeux qu'un spécimen très-incomplet, puisqu'il décrit cette plante comme n'ayant point d'aiguillons. Buberstein parait en a voir apercu l'inexactitude, et il la corrige avec raison.

Les serratures sont toujours doubles.

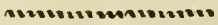 \\ $51^{\circ}$. ESPE்CE. - ROSA CANINA.}

R. Foliolis rigidis, ovatis; ovariis 20-3o. Lindu. Mon. p. 98.-Pnosv. Sonm. p. 36 .

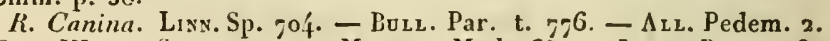
r39.-WiLld. Sp. 2. 1077. - Moensch. Meth. 689. - LaWr. Kos. tt. 6o

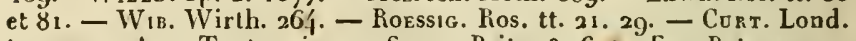
t. $299 .-$ Arz. Tent. prim. - SMirn. Brit. ${ }^{\circ}$. 6. - ENG. Bot. $99^{2} .-$ Svensk. Bot. t. 29. - GMel. Bad.-Als. 2. 422 . - Brat. Lus. 1. $340 .-$ Boв. Taur.-Cauc. 1. 399. - Schrancr. Mon. c. fig. - Fr. dan. t. 555. - Smith. in Rees in I.-Woods. in Act. Jinn. 12. 223. Rav.-Enum. 71.

R. Dumalis. Bechsт. Forstb. 241 et 939 . ex. Piad. L'églantier. Regn. bot. c. fig. Suppl. 29. - RiEDOUT. Ros. 2.9. t. 3.

R. Andegavensis. Bot. Maine-et-Loire. 189 .

R. Glauca. Lors. in Desv. journ?

il. Arvensis. Schranck, Mon. c. fig. 
R. Glaucescens, Mler. Par, 193, Id.-Canina, var, C. ed. 2. p. 362.

R. Nitens. MER. L. c. Lll. - Canina. Var. 6. ed. 2. 362.

R. Teneriffersis. Doos. Cant. ed. 8. 169 .

K. Senticosa. Aenar. liongl. veteask. acad. Haudl. 34. 91. t. 3.

R. Surculosa. Woods. in Aet. liun. 12, 228.

R. Sarmataced. IVoons. in Act. linu. 12. 213.

R. Nuda. Woods. L. c. 12. 205.

R. Afinis. RAv. Enum. 79.

R. Glaucophrlla. IV inch. Ess. geogr. 45 .

Var. 6 Aciphylla pumila. Foliis utrinquè impubibus floribusque multò minoribus.

R. Aciphylla. Rav. Enum. 66 c. fig.-Redotr. Ros. 2. 31 . t. 3.

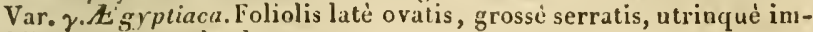
pubibus; receptaculo elongato.

R. Indica. Fonsא. p. cxill?

Var. \& Collina. Foliolis infra. v. petiolo hirsutis; sepalis pedunculisque hispidis; disco conico.

R. Collina. J $\mathrm{ACQ}$. Austr. 2. 58. t. 197. - $\Lambda_{\mathrm{LL}}$. Ped. 1. I fo.-WLLLD.

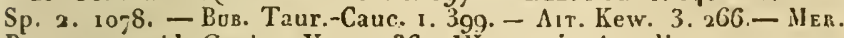
Par. 191. - ill. Canina. Var. \&. 362. Woods. in Act. lina. 12. $219 .-$ Ru. Enum. 163. - Redout. Ros. 2. 13. t. 5.

R. Unbellata. Lexser. Pal. 433 .

R. Fastigiata. Dat. Maine-et-Loire. Suppl.3o.-Dfrarn. Suppl. 535.

R. Platyphylla. Rav. Enum. Sa.

R. Psilopliylla. Rad. L. c. 19נ.

R. Solstutirlis. Eesser. Galic. primit. 1.324.

Var. \& Dumetorum. Foliolis utrinquè hirsutis ; sepalis pedunculisque glabris.

R. Sepium. Bоккн. Forstb. 1527. ex Ruv. Enum. $9^{\circ}$.

R. Dumetorum. Th. Par. 250.- Decand. Suppl.53ł.-Raz. Eaum. 85. - WOods. in Act. linn. 12. 217.

R. Corymbifera. Gmez. Bad.-Als. 2. 424 .

R. Leucanilea. Lois. Nat. S2. - BAT. L. c. 32. - Mer. Par. 193. Id. Var. H. ed. p. 263. - DC. Sappl. 535. - Redour. Ros. 1. 129. t. 49 .

K. Obtusifolia. Desv. Journ. 2. 317.

R. Leucochroa. DEsv. L. c. t. 15. - DC. IJort. Monsp. 138.

R. Siylosa. D. Desv. l. c.

R. Braclescens. Woons. in Act. linn. 12. 216.

Var. '. Casia. Foliolis cæsiis, utrinquè pilosis; tubocalycis elliptico.

R. Casir. ENG. Bot. t. 2367. - Smith, in Rees in 1. - Woods, in $\Lambda$ ct. linn. 12. 212.

R. Vilzosa? Thollz. Par. - R. Camina tomentosa des pépinières.

Se trouve dans tous les lieux incultes en Europe et dans le nord de l'Asie où elle change de forme, selon le sol où elle croît : à Ténérifle (Masson); var. 6 à Wurtzbourg. (R $\mathrm{R}_{\mathrm{A}}$ ) et probablement dans d'autres lieux stúriles. Var. $\gamma$ en Egypte (FonsKHaL). Var. $\zeta$ montagnes di norl de l'Écosse (Boriver Jackson); et à Fontainebleau (TuUnllier). Var. $\delta$. hauteur de Sévres. $i d$. ( $V$. v. Air, et s. sp.)

Buisson de six à sept pieds (et davantage). Branches vertclair, d'un brun rougeâtre du côté du soleil, armées d'ai- 
guiltons forts, épais, en hamecons presque égaux, rareınent iroits et alor's pressés l'un contre l'autre et sans soies. Feuilles distantes, pâles ou vert-foncé, souvent teintes de rouge, et (exposées au soleil) ordinairement cautérisées à leur surface supériemre, sans pubescence. Stipules dilatées, un peu réfléclies et portant une pointe aiguë. Pétioles armés de quelques petits aiguillons crochus. Fotioles de 5 à 7 , ovales ou oblongues, aiguës ou arrondies, sessiles ou presque sessiles, plates ou concaves, unies ou ridées grossièrement, ou finement dentées, on simplement ou doublement. Scrratures tonjours aiguës, sans glandes et convergentes. Cimes d'une nu plusicurs fleurs. Bractées ovales, lancéolées, pressées, aiguës, concaves ou plates, finement dentées et glanduleuses à l'extrémité. Pédonculcs et calices glabres. Tube ovale.Sépales étalées, pointues, caduques, quelquefois divisées. Pétales obcordés, concaves. Disquc épais, élevé. Ovaircs de 20 à 30. Styles presque glabres, séparés, renlermés on peu saillans. Fruit ovale, oblong, écarlate, luisant, saus fleur (1). Péricarpe gros, de forme irréguliere.

On ne trouve point dans tont le système vígétal un exemple plus frappant de caracières insignifiaus pour déterminer une espèce que dans la liste des synonymes qui précèdent. Il n'est pas surprenant que l'espece la plus commune du genre, dont le fiuit est à peine unar avant d'a voir été dévoré par les oiseaux et déposé par eux dans toutes les variétés de sol ct d'expositions possibles, soil susceptible de prendre des différences considérables dans son habitude générale. Les écrivains modernes ont constitué des espèces sur de semblables différences, lesquelles, dans de's genres moins variables, auraient i peinc fixe l'attention du botaniste. On a pris en considération la pubescence selon son absence, sa présence et sa quantité. Lesrosescollina, dumctorum, le canina des auteurs et le bractescens de $\mathbf{M l}$. Woods sout séparés les uns des autres; et cependant que l'on examine un instant le canina comme on le trouve dans toutes les haies, et l'observateur attentif verra sur la même plante quelques feuilles entièrement nues, et d'autres sur lesquelles la nervure principale, et d'autres, les plus prononcées, sont garnies de poils sur la partie inférieure. Voila donc un acheminement à la pubescence qui,en augmentant

(1) Filhout any bloom. Duvet coloré qui tapissait l'extéricux du finit. (JOFFRIN.) 
en quantiti, distingue le collina. Celui-ci a ordinairement des poils à la surlice supérieure de ses jeunes feuilles et aucun sur les plus anciennes. Dans le dumetorum il y a encore augmentation de pubescence qui recouvre l'une et l'inutre surfice des leuilles et qui, devenant très-épaisse et permanente, peut former le bractcscens de Woods. Quant au caractére établi dans cette espèce sur la double ou simple serrature, j'a voue franchement que je suis incapable de l'entendre; j'ai essayé de tracer une ligne de démarcation entre eux, mais sills succès; ils n'ont point de limites ; eitr personne ne peut dire avee assurance si les serratures de telle feuille sont simples ou doubles. Mlias la valeur de ees caractices et d'autres semblables a déjà été mise en discussion; il est donc superlu de les examiner de nouveau.

La description qui suit s'applique rigourensement an canina var. A. Lorsque celui-ci est fiaible et qu'il cruît dans les bois ou les lieux ombragés parni les gramiuées, il porte des aignillons droits et devient le nuda de Woods. Arec des aiguillons plus écartés, c'est l'andegavensis de H. Batard; lorsqu'ils sont très-épais, il est le canina of de Rau. La tige est légèrement sétifère dans une plante du jardin de Ml. Sabine. Une autre de la même collection a ses folioles bipinnées. Les lolioles sont tristes (dull) dans le surculosa ou li canina 3 de Wouds, firt arrondies et à serratures obluses dans une plante de M. Lyell. Lilles sont inégalernent dentées dans le surculosa de Wonds et l'affinis de Rau; pubescentes à la surface supérieure dans l'affiris. Les sépales sont érigées et disposées à la persistance dans le glucophylla de H. Winch, presque simples dans le canina D. de Woods, et glanduleuses en dehors dans le glaucophylla. Le disque est plat dans le surcalosa $\beta$ de M. Woods. Le fruit est presque rond dins le canina e et le: surculosa sde Woods, tandis quil est triss-petit dans le teneriffensis de Doon, et rude dans le canina $\beta$ de Woods et l'andegavensis de Batard, Dans ce dernier les pédoncules sont hispides. Tel est le canina le plus eommun et celui que Linni connaissait le mieux. La planie de Tenériffe est sensible au froif, fleurit pou ct produit de petits liruits différens. Le glaucopinylla de i. W. Wincls est une variété reinarquable par son fruit obovale et ses sépales persistantes.

Le caractere de la var. $\beta$ aciphylla semble consister en une proportion moindre dans toutes ses parties, apparence qui n'est pas ralle dans ce pays quoique je ne l'aie jamais re- 
marquée au degré que le signe de Rau la présente. Le spécímen figuré par Redonté montre évidemment un pas de plus. vers une croissance plus vigoureuse. Peut-être cette plante estelle au canina ce que le rosier nain de Chine cultivé dans nos jardins est au $R$. indica.

Je ne connais la var. y agyptiaca que par un échantillon de l'herbier de S. Jos. Banks, de Forskahl, marqué aegyptiaca. Il se distingue par la profondeur peu ordinaire des serratures dans ses leuilles, et son très-long réceptacle. Forskahl ne parle d'aucun rosier qu'il ait trouvé en Égypte; serait-il donc colui qu'il appelle indica et qu'il a apporté des montagnes de l'Arabie-Heureuse?

La var. $\Delta$ collina a le revers des feuilles velu, le dessus luisant, les sépales et lespédoncules ordinairement hispides. Sous aucun autre rapport il ne diftère pas du canina a. Le canina de la Bot. ang. appartient a une plante très-différente qui est le systyla. Le platyphylla de Rau a ses pédoncules glabres, tt le psylophylla du même auteur a les pétioles cotonneux 't la surface inférieure nue jusqu'aux feuilies. Le fastigiata de Bataud n'a aucun caractère qui puisse servir à le faire distinguer?

Quant i la var. \& dumetorum de Thuillier, on peut consulter la liste des synonymes suivans : il se distingue du canina \& par sa nuance d'un gris triste, occasionée par l'épaisseur de sa pubescence dans toutes les parties des leuilles et pas autrement. Il est aussi commun dans le nord de l'Angleterre qu'ailleurs. Les pétioles sont quelquefois sans armure comme dans le sepium de Rau. Le teucochroa a les styles un peu saillans et unis par leur duret. Il faut prendre garle de confondre celui-ci avec le stylosa dont les styles sont glabres et par conséquent sont cohérens par quelque autre cause que par l'intexture de leurs poils. J'ai des échantillons, qi:e je dois à 11. Lyell, d'un rosier très-gris qui se troure a Kinmordy avec des sépales presque simples, qui doit se rapporter ici. Le bractescens de Woods a de très-courts pédoncules et de grandes bractées; mais j'ai examiné le spécinten de MI. Woods dans la collection de la société linnéenne sans pouvoir le distinguer du dumetorum. Chaque diversité se forme dans les bractées, et la longueur du pédoncule peut se remarquer dans les canina d'Ecosse ì feuilles velues.

La var. Ł. Cossia cst une plante remarquible dont il est fait mertion pom la premiere fois dans la Botanique Anglaise. Il 
est difficile de la trouver ailleurs que dans les montagnes d'Lcosse, où elle est encore fort rare. Sa teinte glauque la distingue suffisamment.

Il y a une particularití singulitre dans le canina, qui est que les styles sont plus velus en raison que cette plante est recueillie dans le Nord. Ils deviennent glabres à mesure qu'on les recueille au Sud. Les poils disparaissent totalement de ces organes sur les individus pris a Mladère. Les longs rameaux epar's servent quelquefois aux mêmes objets que ceux du rubus fruticosus; mais ils lemr sont infërieurs étant plus fragiles. Les Tartares font du thé avec ses sommités et ses f:uilles; quelques liusses en font aussi le même usage, notamınent en Siberie, où ils vantent beaucoup ses vertus stomachiques. Ceux du Volga tirent de ses fleurs une liqueur. spiritneuse par lit fermentation. Dans l'Ukraine on les confit an miel et au sucre.

"Nola. Parmi les especes de rosicrs qui présentent le plus d'aberrations, aucune ne peut se comparer au canina, et l'on doit louer la circonspection avec laquelle M. Lindley a limité des variétés tris-rapprochées, dont on a voulu farre des espéces distinctes. l'eut-être nos botanistes modernes ne seront-ils pas satisfaits de ce travail ; maisil ètait indispensable pour jeter quelque clarté dans sa nomenclature spécifique si nombreusir. MM. Desvaux et Mérat ont eux-mêues senti l'inconvénient de l'abus des espéces en replacant comme variétés celles qu'ils avaient constituées d'abord. Hais que l'on ait voulu établir une classification du genre sur le nombre et la forme des serratures, c'est ce qu'il y a de plus surprenant; ce caractère accessoire et assez vague serait bon tout au plus à classer d.s variétés cultivées, telles que celles du centifolia et du gatlica, dans lesquelles je pourrais distinguer trois et quatre sortes de serratures. Je ne parle de ce systeme qu'en passant, et je doute qu'il puisse jamais être adoptí. "

\section{$52^{\circ}$. ESPECE. - RUBRIFOLIA.}

R. Aculeis parvis, distantibus; foliolis ovatis ramisque glaucis, opacis, discoloribus; ovariis $20-30$. LaND. Mon.

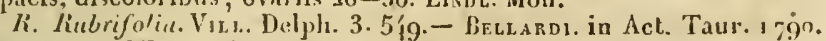

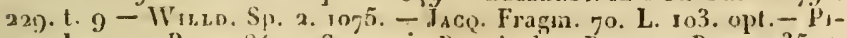

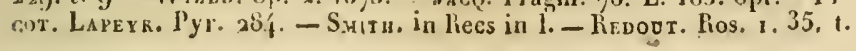




\section{$10 \%$}

4. - Linol. in Bot. veg. t. 3fo.-Provr Mon. p. 35.-Id. Somm. p. 38. R. n. 1ror. 6. HaL s. Ilelv.

R. Multifoin. Rexn. Met. Lans. r. -o. t. 6.

R. Rubricauda. II LL. Fil. in Ross. Areh. b. r. st. 2. p. 6.

R. Canina 6. Suter. lielv. 1. 302.

R. Glauca. Desf Cat. H P. 175 .

R. Glaucescens. Wolf, in Ross. Arch. 3. 3-6

R. Lurida. A ппв Ews's lioses

R. Cinnamomea $\gamma$. Riedout. Fios. $1 \quad 134$

Se trouve dans les bois près de Lans (VIL zens); dans les $\Lambda$ pes sabaudiennes (Brllardi); en Suisse (H H Ler); en Autriche à Guttenstein (JacQuin); aux Pyrénées (LA Pérouse); montagnes d'Auvergue (Redauté). ( $V$. v. c. et s. s[1.)

Tige rouge-foncé ou pourpre, couverte d'une substance farineuse (telle qu'on la voit sur les prumes), pâle et armée d'aiguillons égaux, petits, courts, pâles et courbés, qui sont tous épars, mais pas plus grands sur les rejetons. Feuilles teintes de rouge, trés-glanques, ridées, opaques. Fleurs petites, rouge-foncé. Disque lort épaissi, fermant presque l'orifice. Fruit oblong; pulpe fort tendre. Sous les autres rapports, les caractères du canina, dont elle diffère néanmoins par les caractères et son apparence. Si l'on faithien attention àlatriste nuance rougc-glauque de ses branches, i leurs petits aiguillons et aux longues sépales, on ne la conloudra jamais arec le canina. Elle a été étrangement rapportée au cimamomea par H. Thory. Quelle en est la raison? c'est ce qu'il ne in'est pas possible de conjecturer.

Nota. "Probablement parce que la tige et les branches sont fortement colorées, et qu'il se trouve quelques aiguillons stipulaires géminés. Mais ces considéralions ne sont pas d'un assez grand poids pour distraire une espèce qui appartient aux canines à tant de litres."

\section{ESPECCE. - R. MICROPHYLLA.}

R. Foliolis ovatis. minoribus; bracteis appressis, pectinatis ; fuctu aculeato. Lindz. Mon. p. 19. Adr.

$R$. Microphylla. Foxв. Fl. ind. ined.

Uai-tong-liong Sinensium.

Se trouve en Chine. Poxburg, IVallich. ( $V$. s.sp. H. Lameri.)

Pelite plante compacte, d'un vert clair. Branches grêles ct souvent fl:xibles. Aiguillons stipulaires droits. Stipules très-étroites, ilargrit's au sommet. Pétioles un peu aicuillon- 
nés, très-faibles. Folioles 5-9, tris-petites, luisantes, arrondies, ovales, aignës, sans amenne pubeseence et finement denlées. Fleurs solitairès, avec une tractée étroite, aiguï, rouge pâle, très-doubles. Calice tout couverl d'diguillons droits, trèsserrés. Tubes ronds. Sépales très-convertes, dilatées, poinlues, cotonneuses sur les bords, forme de ceuxdu bracteata.

Charmant petit arbuste ressemblant dans sa forme générale à celui de Macartney, et particulierement dans la lorme des divisions de son calice. Il differe de tons ceux de la tribu par son calice excessivement muriqué at par ses stipules étroites.

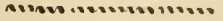 \\ 54. ESPECE. - ROSA SERICEA.}

IV. Aculeis stipularibus compressis, superioribus runcinatis; foliolis oblongis, obtusis, apice serratis, sublùs sericeis. Livd. Mon. p, 105 c. fig. - Prosv. Somur. p. 38.

Se trouve dans le Gossan-Than. W Walich. ( $V$. s. sp. m. Herb. Banks. LINDL.)

Branches brunes, paides, droites, les anciennes très-ridées. Aiguillons très-grands, ovales, comprimés, leur pointe tournée vers le ciel, plus pressés sous les stipules. Teuilles très-rapprochées. Stipules longues, étroites, concaves, sans pulescence, nues et frangées ì leur extrémité, aiguës et dentées au sommet. Pélioles légèrement cotonneux ou uus, sans armure ou garnis de quelques aiguillons droits, dont la base est large, et de quelques soies. Folioles de $;$ d̀ 11 , oblongues, aplaties, vertes et nues en dessus, plus pâles, et les nervures principales soyeuses en dessous. Leur extrémité, qui est obtuse, simplement et profondément dentée, et les serratures aiguës. Le pétiole dans quelques échantillons est extraordinairement aliongé avant que les premières folioles l'atteignent. Fleurs solitaires, concaves, sans bractées, droites ou penchées. Pédoncules et calice nus. Tube ovale. Sépales ovales avec une pointe trés-étroitc, légírement pubescente.

- C'est le premier d'une séric d'espèces trouvées dans les climats bralans de l'Asie, mas qui ne s'éloigne pas essentielle-

(1) Lc mot soyeux dont jai fait usage jusqu ici, a maintenant et pour celte espice une autre signicication; il est pris ici pour le lissu satiné et reloutéde la soie. 
ment des caractères assignés à cette tribu. Il est remarquable par la texture soyeuse du dessous de ses létilles oblongues, obtuses anx extremités, et profondément dentées dans cette partie.

Il a été découvert récemment au Gossan-Than avec le macropylta trouvé dans le même canton. Il forme le châ̂non entre les roses de l'Inde et celles de l'Europe. Les échantillons d'après lesquels ma description et la figure ont été tracées appartiennent à la ricle collection de S. Jos. Banks.

\section{5". ESPECE. - ROSA INDICA.}

li. Foliis ellipticis, acuminatis, subbasatis, acute seratis; ovarios 4050.

Var. a. Vulgaris. Fructu turbinato.

R. Cleinensis. Var. E. Cansan. Jronv. Nom p. 105.

R. Seniperflorens carnea. Roess. lios. t. ig.

Var. R. Oduratissima. Fructu ovato; floribus odoratissimis.

K. Odoratissima. Swelt. Hort. sub. Lond.

R. Indica fiagrans. Pindout. hos. 1. 61, t. I9.

Var. n Pumila. Fruticulus omniparte minor. Linda.

R. Indica pumila. Hedout. Kos. 1. 115. t. 42 .

Se trouve à Ja Chine, près du golfe de Cantou (Staunton.) ( $V$.v. cult. et s. sp. in herb. Bavks, LindeEy.)

Branches fortes, d'un vert clair, armées d'aiguillons bruns, épars, 'comprinés et crochus. Fcuilles un peu luisantes, d'un vert clair, plus pâles et pubescentes en dessous. Stiputes très-étroites, subulées et un peu glanduleuses en leurs bords. Fotioles de 3 ì 5 , presque égales, elliptiquesaiguës, funement dentées, les serratures deviennent crenclées dius les feuilles anciennes, vert-clair en dessus, plus pâles "t légèrement veloutées en dessous. Filears solitaires ou 2 à 5 au plus grandes, semi-doubles, carnées. Braclées presque mulles. Pédoncules, scabres, allongrés. Tube du calice ovale, turbiuć, court, nu. Sépalés presque simples, velues, quelţuefois persistantes. Pélales lauges, concaves, sans échancrures. Elamines nombreuses, environ 120. Disque épais. Ovaircs de 40 à 50. Styles saillans, presque nus, séparés. Fruil turibiné, aplati, trés-gros, écarlate ou pourpre foncé.

11 r'est peut-être plus temps dis deuander ce que linné a voulu désigner par indica puisque son caractère spécifique et sa description ne conviennent à ancune des espéces indigenes -de liı Clibe, du moius à celles qui composent cetle tribu. La 
figure de Petirer sur laquelle il se fonde et en quoi il a écé suivi par Willdenow, Poiret et plísienrs autres, appartient à une plante bien diflérente qui a beacoup de ripport avec le Banlisia et que j'ai appelée microcarpa. J'ai toute fois examini les échantillons de Linné et je ne doute pas qu'ils n'appartiennent à cette espéce; mais ayant étudié pareillement le spécimen dont S. James Smith forme le type du $R$. sinica (estce celui de Linné ou celui de M. Lindley?), je n'hésite pas à prononcer que cet échantillon est une monstruosité de l'indicı de nos jardins. Les stipules sont étroites, pointues, finement dentèes en leurs bandes; les aiguillons droits, faibles, inégaux, ce que l'on doit trouver dans l'indiea, et ne convient pas au fort ou a l'état laible du sinica. Ce nom m'inant paru suranné, je l'ai réservé pour la plante qui le porle dans l'Hortus hervensis.

La Rose de Chine odorante (odoratissina ), dont le parfum est si agréable, est une varièté dont les fruits sont ovales. La plante est des plus petites. Les cultivateurs doivent savoir qu'il y en a deux variètés: l'une, la plus ordinaire, est d'une odeur faible et moins suave que l'autre, dont la propagation cst plus dillicile.

Nota. "Si l'on compare celte monographie à celle de M. Lindley, on s'apercevra facilement que la description du $R$. indica n'est pás la même; elle s'applique au $R$. semperflorens, quoiqu'il ait danné ce nom à une autre plante comme on le verra tout à l'heure à l'art $R$. chincnsis. Je stipprime ce que dit M. Lindley au sujet de la variété longifolia, qui me paraît, comme à $\mathrm{M}$. Thory, appartenir an semperflorcus, que d'ailleurs l'éditeur des Roses de liedouté appelie indica, ainsi que II. Lindley.

\section{$56^{\circ}$. ESPECL. - ROSA NOISETTIANA. Busc.}

R. Folinlis ovatis, acutis, simpliciter serratis; pedunculis rillosis; tluribus numerosis, umbellato-corymbosis.

R. Merlica.

Var. Norsetrian. Renoct. Ros.

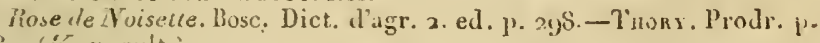
I3 . (ir. v. cult.)

a Buisson serri, ilevé de 3 i 5 p. Brancines d'un vert bunâtre. Aiguillons furts, épars, crochus. Feuilles d'un vert brillant, assez rupprocluces. Stipules très-étroites, i 2 segmens 
subulés, cilices finement en leurs bandes. Folioles presque toujours $\%$, ovales-aiguës, finement denties, serrutures aiguës, sans pubescence, vertes et un peu luisantes en dessus, plus pâles en dessous. Pétioles armés de petits aiguillons courts, en fir de faux, et velus. Fleurs nombreuses, moyennes, doubles, couleur de chair. Pétales entiers. Pédoncules, tubes du calice et sépales relus; ces dernì̀res simples, larges à leur base, aiguës et non dilatées à leur sommet et réféchies. Braclées lancéolées, cunıares, ciliées en leurs bords.

* Ce rosier qui fait l'ornement de tous nos jardins, a été considéré par M. Thory comme une liybride de l'indica et du moschata. Il serait possible qu'il le fat de la première avec notre multiflora, qui peut-ĉtre lui-même n'est pas le véritable multiflora de 'Thumber, mais le florida d'Aiton. Quoi qu'il en soit, je me suis décidé à cause de ses caractères, quile rapprochent de cette tribu, tels que les serratures convergentes, les sépales rífléchies. Je conviens ne l'avoir pas vu multiplié par les graines; mais la culture en obtiendra probablement des individus presque simples el qui produiront des fruits mars, car ce rosier est déja presque naturalisé dans le climat de Paris.

- C'est M. Philippe Noisette qui l'a envoyé des États-Unis à son frère en $18 .$. , ainsi qu'à $M^{*} \ldots . .$. , pépiniériste à Rouen. 11 était convenable de donner à ce Rosier le nom d'une famille de cultivateurs aussi distingués par leurs connaissances horticulturales.”

\section{$5 \%$ F. ESPECE. - ROSA SEMPERFLORENS.}

R. Foliolis ellipticis, acuminatis, glabris, cruato-serratis ; sulutus glaucis; pedunculis glabris; orariis $20-30$.

Var. z. Vulgaris. Fructu ovato.

R. Chinensis. Willd. Sp. 2. 1078 .

R. Bengalensis. Pe.r. Syn. 2. 3o.

R. Indica. Linde. Monogr. p. 106.

K. Indica. Tie doe t. Ilos. 1. .. 1. t. 14. - 2. 35. 1. 35.

R. Semperforens. Prosv. Fomencl. p. $10 \mathrm{r}$.

R. du bengule. Вот. culi. 3. 3 I.

R. Flemiri. Poir. Enc. ro.

Var. R. Diversifolia. Frirtu elongato.

R. Diversifolia. Vent. Cels. t. 35 .

R.Semperforens. Livol. Wnn. p. ios.

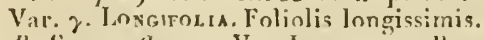

f. Semperflorens. Var. Longifolia. l'Roxp. Nonı. p. M1.

R. Indica. Var. m. Luscirolia. Lixde. Mou. P. IC.G. 
R. Longifolia. WILLd. Sp. 2. 1067. - hedout. Ros.22. 27. t. 12.

Se trouve a la Chine et au Bengale. $Y$. v. cult. )

Arbuste très-vigoureux, italé. Branches furtes, d'un vert clair quelquelois un peu rougeâtre, armées d'aiguilions presque triangulaires, erochus, comprimés, un peu bruns, épirs. Stipules trés-étroites, à 2 segmens suluulés, étant ciliées tt glanduleuses en leurs bords. Fétioles presyue glibres, mais arués de petits aiguillons courts, distans. un peu crochus. Folioles de 5 a 5 , inégales, elliptiques, lancéulées, tendres et simplement dentées dans les jeunes rameaux, coriaces et erénelées dans celles de la base, vert-foncé en dessus, un pea ylauques en lessous, parfaitement glabres. Fleurs solitaires vu de 2 d 5 , ordinairement semi - doubles, l'un rouge clair, inais peu éclatint; odeur presque nulle. Pélules souvent échancrés. Bractécs étroites, lancéolées, sans pubescence, glanduleuses et ciliées. Pédoncutes lungrs et giabres ainsi que le tube du calice ovale. Sépalcs simples avec une pointe un peu élargie au sommet, et velıes en leurs borls. Étamines de 80 à ıo. Disque épais, en cône. Ovaires de 20 à 5o. Styles presque nus, saillaus, très-rrêles, séparés. Fruit ovale, un peu turisiné (étroit à sa base), couronné par les sépales réfléchies, ćcarlate.

* Il me semble que la descripion détaillée que je viens de faire de ce rosier suffit pour le distinguer de l'indica, yuoiqu'ils soient tres-rapprochés; mais celui-ci est infuiment plus vigoureux, plus rustique, plus florifëre. Iltporte deux caractéres bien prononcés, comme le dit avec raison M. Poiret, dont l'un est de produire des fleurs sans interruption pendant toute l'année, la mauvaise saison exceptée, l'autre d'avojir la plupart de ses feuilles ternées ou géminées, mais dont les deux folioles inférieures sont très-petites et l'impaire du sommet trèsgrande.

- Le semperflorens de Willdenow semble s'appliquer à une espèce des Banksiance, le sinica de Lindley qui est le terrata de Bosc.

„La variété 6 diversifolia est très-singulière. C'est peutêtre une monstruosité. Elle porte plus de feuilles ternées que de quinées et jusqu'à des feuilles simples; elles sont dentées et non crénelécs. Tube du calice ovale-oblong, les sépales prolongées à leur pointe en une foliole dentée. Cette variété appartient bien certainement a notre semperflorens et non a l'indica de M. Lindley. 
n J'en dirai autant de la variété y longifolia. Ses feuilles ressemblent à celles dia saule; mais les pètales sont aussi singulières que les feuilles, car ils sont linguiformes; les sépales très-étroites terminées en pointe aiguë. Les tubes sont aussi prolongés relativement aux autres parties de la plante, qui n'est que de collection.

"Le croisement des graines de cette plante arec l'indica, le chinensis (voyez plus bas) et d'autres espèces, a produit ce grand nombre de variétés hybrides que l'on admire dans nos jardins, et dont parle 11. Lindley à son article Semperflorens. C'est à 'Mlonza en Lombardie, à Rennes en Bretagne, dans les pépinières du Luxembourg, de Chenevières, etc., que l'on a multiplié ces variétés d'ıne manière surprenante; on les voit presque toutes réunies dans la collection de M. Noisette à Paris.

- Je ne dois pas oublier un rosier que $\mathbf{M}$. Hardy cultive au Luxembourg, et qu'il a reçu d'Amérique sons le nom de chamnagana. Ce nom aurait pourtant plus de rapport aves ceusde sibérie. Il tient du semperflorens et du noisettiana. mais plus de ce dernier par ses fleurs et les parties de la fructification; mais il faut remarquer que les corymbes sont axillaires. Les folioles de 5 à $z$, ovales, aiguës, crénelées autant qu'incisées, vertes en dessus, pâles en dessous. Les aiguillons rares, courts et crochus. Peut-être cette plante, dont les fleurs sont semi-doubles et carnées, est-elle le type du noisettiana; c'est ce que d'itératives observations pourraient éclaircir.

\section{LSPÈCE. - ROSA LATITRENCEANA.}

R. Nana. Foliolis ovatis, lanceolatis, argutè serratis, subtùs glaucis; petalis acuminatis ; ovariis $7-8$.

R. Semperflorens minima. Suxss. Bot. mag. n. 1762.

li. Pusilla. Madrutics. Cut. n. i5.

K. Lanrenceana. Swetr. Hort. sub. Lond.

Se trouve vraisemblablement en Chine. ( $V$. v. cult.)

Arbuste très-petit, serré, qui s'èlève rarement à un pied; ses aiguillons sont larges, forts et presque droils. Folioles ovales, lancéolées, fort aiguës, finement dentées, conniventes, vertes en dessus, glauques en dessous, sans pubescence. $P \dot{c}-$ tales petits, couleur carnée, limbe acuminé vers le milieu. Dvaires $z-8$. D'ailleurs les caractères du semperflorens, dont 
cependant je crois devoir lc séparer. La difference du nombre des ovaires dans cette tribu paraît constatée, ce qui lui donne de l'importance. M. Swelt l'a apportée de l'île Maurice (de Bourbon) il y a quclques années, et peut-être n'est-ce que le pusilla du catalogue du jardin botanique te cette île.

La Chine est probablement la patrie de ce joli rosier; à raison de ses rapports avec le semperflorens, c'est lui que nos jardiniers appellent Bengale pompon; mais il ne supporte pas encore les hivers rigonreux, et si ce n'étaient les différences assez notables que nous avons présentées, sa faculté de produire comme la première des fleurs pendant toute la belle saison pourrait faire croire qu'il n'en est qu'une variété. "C'est probablement M. Swelt qui l'a dédié à mademoiselle Lawrence, auteur d'une belle suite de passiflora et d'autres plintes dessinées et coloriées d'après nature. M. Noisette possède dans sa collection le tazurenceana à fleurs simples."

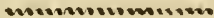

\section{ESPËCE. - ROSA CHINENSIS.}

h. Humilior; foliolis ovato-lanceolatis, arguté serratis, sulbtùs rubro coloratis; petalis integris; ovariis 15.

Ii. Chinensis. JAcQ. Obs. 3. 5. t. 55. - Bosc. Dict. d’agr. p. 26 '. Lawr. Kos. t. 28. - Pronv. Nom. p. 104.

R. Semperflorens. IVILx. Sp. 2 1or 8. - Idem. Var. Sinfinsis. Pers. Syn. 2. 42.-Mart. in Mill. Dict 34.-Prorv. Somm. p. 39. - Livdr. Mon. p. 108.

R. Indica. Redoot. Ros. 1, 49, t.13, 123.t. 46.2, 27, t. 16.

Se trouve a la Chine (ÉkeberG). ( $V$. v. cult. et s. sp. Lindér.)

Arbrisseau étalé, élégant. Branches faibles, d'un vert prononcé, armées d'aiguillons épais, counprinés et crochus, et de quelques glandes. Feuilles luisantes, distantes. Stiputes étroites, plates, glanduleuses et légèrement soycuses. Fotioles de 3 à 5 , ovales, lancéolées, simplement et lortement dentées, quelquefois crénelées, minces, plates en dessus, plus pîles en dessous, arec une forte teinte de pourpre, la paire inférieure petite, mais pas autant que dans le semperflorens, et manquant surtout aux extrémités des rameaux. Fleurs solitaires, cramoisi-foncé. Bractées étroites, lancéolées, dentées et frangéts de glandes. Pédoncules très-longs, scabres avec de petites glandes. Tube du calice oblong, nu. Sépales réfléchies, caduques.Disque coniqne, épais.Ovaires 
15. Stytes très-grêles, presque nus, saillans, sèparés. Pétales entiers. Fruit sphérique.

Celte espèce est remarquable pour être du nombre de celles dont les étamines tombent presqu'en même temps que les pétales, ce qui ne se trouve point applicable à l'état semidouble de l'indica. On peut le distinguer de celui-ci par ses branches plus faibles, ses fleurs d'un rouge foncé, ses feuilles plus nombreuses, et ordinairement panachées de cramoisi. II y a aussi une différence dans le nombre des ovaires qui ne dépasse guìre celui de 15 , tandis que le fruit de l'indica en renlerme 40 à 50.

Nous avons plusieurs magnifiques variétés à fleurs semidoubles crimoisies dans nos jardins. Les Français paraissent en avoir quelques-unes beaucoup plus belles qui ne nous sont. pas encore parvenues.

Nota. "Il y a certainement ici une confusion de noms. La description que fait M. Lindley de son R. semperflorens convient si bien à notre chinensis que je n'y ai presque pas changé un seul mot; car c'est une petite plante délicate à fleur's semi-doubles cramoisies, qui ne fleurit qu'une fois dans l'année; ce qui ne peut s'appliquer au semperflorens des Françis, arbuste vigoureux dont les fleurs d'un pourpre clair se renouvellent presque sans interruption, et qui ne sont pas solitaires, du moins pendant la culture. Alurs plusieurs variétés de l'indica (Lind.) doivent se rapporter au semperflorens, et surtout le tongifolia. Ainsi M. Thory n'avait point tert de rapporter le tongifolia au semperflorens, quoiqu'il ait réuni celte espèce à l'indica de Linnous, que nous ne pouvons adopter d'après les phrases, lesquelles, comme nous l'avons dit, se rapportent à d'autres plantes.

"C'est probablement des semences du chinensis el de quelques croisemens que sont sorties ces magnifiques variétés cramoisies qui présentent des couleurs si vives. Les plus belles dans ce genre sont venues de liennes, et sont. cultivées à Trianon par M. Barrier, concicrge de ce château royal.»

\section{TRIBU. - SYSTYLAE.}

Styli in coluınnam elongatam cohærentes; stipulæ adnatx, Obs. Leur port est à peu près le même que celui de la deraicre tribu; les feuilles sont presque toujours vertes. 


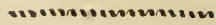 \\ 60. ESPËCE. - ROSA SYSTYLA.}

R. Surculis assurgentibus; aculeis validis, aduncis. Lindu. Mon. p. 111 . Pronv. Somm. p. 40.

Var. a. Ovata. Foliolis ovatis; fructu oblongo.

R. Collina. Eng. Bot. n. 18,5 . - Smirn in hees in 1.

R.Systyla.Bot. Haine-et-Loire.Suppl.31.-Woods. in Act. linn.12.230.

R. Strlosa. Desv. Journ. 2. 31 7. - Decano. Ilort. monsp. 138.

R. Brevisyla. D. C. Suppl. Fl. fr. 537. lac. cit.

R. Bibracteata. D C.

Var. $\beta$. Lanceonata. Foliolis ovato-lanceolatis; fructu spherico.

Var. $\gamma$. Mossonı. Caule humiliore, florifero, erecto, multilloro; ramis raro setigeris.

Se trouve, var. a, en Angleterre, en France (Batard et DrcanDoLLE); var. $\beta$ en Irlande méridionale (Drumond); var. $\gamma$ en Angleterre, près IVatfand (Mlle, Munro); et en France, à Attichy. ( $V$. r. cult. et s. sp.)

Buisson qui a l'habitude et la plupart des caractères du R. canina, mais qui en differe principalement par ses styles unis en une longue colonne, et un plus grand numbre de fleurs rassemblées en cime.

La variété $\beta$ fut envoyée à $\mathbf{I I}$. Hooker du midi de l'Irlande, par M. Druminond; elle differe de la première par ses fruits presque ronds, ses longues feuilles luisantes et ridées.

Le Monsonice, variété $\gamma$. qui est une plante charmante, trouvée dans une haie, pres Wallord, par Mlle. Munro. M. Sabine a voulu qu'elle portât le nom de lady Monson, à raison de ce qu'elle fut apportée de ses jardins, d'où elle s'est répandue. Il peut être considéré comme une variété hybride du systyla croisé avec l'arvensis. Il se fait distingruer des deux autres variétés par son habitude plus petite, ses rameaux florifères plus droits, raides et terminés par une cime ou corymbe de fleurs des plus élégans. La couleur de son fruit est d'un rouge orangé plus prononcé que dansle systyla. Nota. M. Leroux m'a rapporté cette plante d'Attichy; mais aux caractères près, qui sont les mêmes, je la crois plus grande que celle de Watford.

\section{ESPËCE. - ROSA ARVENSIS.}

R. Surculis flagelliformibus; aculeis inæqualibus, falcatis; foliolis subtùs glaucis. Lind. Mon. 12. - Pronv. Somm. p. 41.

R. Campestris, repens, alba. ЕАн. Pin. 184. 


\section{4}

R. Sylvestris, etc. Bacr. Hist. 2.24⿰亻

R. Candida. Scop. Carn. 1. 35作.

R. Arvensis. Huds. Angl. cu. 1. 192, - Lisx. Mant. 2. 2f́j. - Alu. Pedem. 2. 139. - VILLD. Sp. 3. $1066 .-$ LaWr. Ros. t. 86. - S Mith. Brit. 2. 538. - ling. Bot. 188. - Pers. Syn. 2. 47- - Ait. Kew. 3. 259. - Sмпт H, in liees in 1. - Woous. in Act. linn. 12.232. - Redout. Ros. 1. Sg. t. 33.-Bot. Mag. t. 2054. - Pronv. Nomencl. p. 21.

R. n. I102. HiLl. Helv.

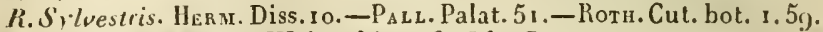

R. Sicandens. Monch. Weis. phl. 118 . fide. Pall.

Ii. Ilerporhoulon. EHr. Beitr. 2. 69.

R. Halleri. Krock. Siles. 2. 150 .

h. Fusca. Moensch. Meth. 688 .

R. Serpens. Eir. Orb. 35. - Wibel. IVerkl, 265.

R. Sempervirens. Rioess. Ros. t. $3_{2}$.

R. Repens. Gmel. Bad.-Als. 2. 418. - Willd. Enuin. 547.-Jer. Fragnı. 69 t. $104 .-$ R $\Delta$. Enum. 40.

Var. B. Mostana. Puinila; fructu hispidulo.

R. Montana. Will. Delpli. 3.547. - Suter. Helv. 1. 300.-Willd. Sp. 2. $40-5$. - Smitu. in liees in 1.

Var. $\gamma$. Hy ERJA. Surculis crassioribus; brevioribus florifero erecto, multifloro; ramis sparsim setigeris; stylis discretis.

R. Mrbride. Scun exich. Cut.

R. Geminata. Kad. Enum. 39

R. Gallica hybrida. SER. Mel. bot. n. 4. p. 39.

Se trouve en Angleterre el en France, dans les buissnns, en Pićmont (Alloni); Palatinat (Pollich); Allemagne (Rотн); Silésie (Kroker); lieux plats de la Suisse (Hooker); var. $\beta$. montagnes du Datuphiné (Villars); en Suisse (Suter); var. $\gamma$. environs de Versailles (Pronv.). ( $V$. v. cult. et s. sp.)

Branches allongées, flexibles on grêles, d'une couleurpresque glanque, un peu triste, armées d'aiguillons égaux, épars, arqués ou droits; ceux des vieilles branches presque blancs, petits, de couleur rouge dans les plus jeunes, et très-souvent nuls dans les spécimens pris sur de faibles individus. Feuilles distantes, d'un vert foncé ou jaunâtre dans un sol caleaire. Stipules étroites, plates, nues, l'rangées de glandes, rouges dans leur milieu. Pétioles pubescens, avec des glandes éparses et des aiguillons arqués, petits, épars et dorsaux. Folioles 5 à 7 , aplaties, ovales, quelquefois ondulées, simplement dentées, très-glauques en dessous, la principale nervure un peu velue. Flcur's solitaires sur les rameaux, nombreuses sur les rejetons, blanches et jaunâtres à la base, ayant peu d'odeur, d'abord en forme de coupe, et plus ouvertes ensuite. Pédoncules scabres, aree des glandes et très-peu de soies. Tube du calice ovale, uu. Sépales courtes, ovales, concares, un peu divisées, ct celles qui lc sont, scabres avec glandes. Pétales 


\section{5}

oblongs, ovales, émarginés. Étamines persistantes. Disque relevé, charnu. Ovaires 15 à 25 . Styles unis en une lon rue colonne glibre. Fruit écurlate, rond ou oblong.

Plante trés-commune en plusiem's parties de l'Angleterre, l'ornement des haies pendant les mois d'été par ses fleurs élérantes de couleur d'albâtre. Ces fleurs ont bien plus la forme d'une coupe que celle du systyla, et certainement beaucoup plus qu'aucune autre fleur indigène. M. Sabine en a une variété à fleurs roses (elles sont carnées, du moins dans ma collection ).

Le docteur Afzélius considère l'arvensis de Linné comme une plante différente de la nôtre, qui ne croît point en Suède. C'est peut-être une variété du cinnamomea qui est figurée dans la Flora danica, sous le nom de fluvialis. L'herbier de Linné ne donne aucun éclaircissement à ce sujet, et je ne trouve rien qui puisse résoudre la difficulté.

Les styles réunis en une longue colonne glabre, indirectement décrits par S. James Smith, comme s'allongeant après la floraison, distinguent celle-ci de toutes les antres especes de la Grande-Bretagne, excepté la dernière. Ses raneaux longrs et traînans, couleur glauque et triste, empreints de pourpre, le distinguent lu stylosa, dont les branches sont d'un vert clair.

Il n'y a pas de doute que l'on ne doive rapporter à cette espèce la rose du comtéd'Ayr de nos jardins (ayrshiverose) (1), comme l'a déji fait entendre le docteur Sims. Il y a pourtant deux variétés de cette mềne plante; l'une qui se vend dans les pépinieres des environs de Londres, que M. Sabine cultive, et que je suis porté à considérer comme la véritable variété de l'arvensis; l'autre, qui se cultive à Kew, vient du sempervirens dont elle paraît ne différer en rien. Cette plante a été regardée comme le véritable ayrshire, et publiée comme telle sous le nom de capreolata, dans le Journal philosophique d'Édimbourg, par M. Neill, qui nous assure que ce nom lui a été donné de l'ruits rapportés de l'Amérique septentrionale, et cultivés au châteaiı de Loudon dans le comté d'Ayr. Sans discuter sur l'exactitude du fuit, je me contenterai de dire que si ces graines ont été apportées de l'Amérique, elles y auraient été originairement portées d'Europe.

(1) "Je l'avais placée aux sempervirens dans le sommaire; mais clle a plus de rapports avec l'arvensis. Elle a fleuri en 1823 ." 
Il n'est pas difficile de distinguer l'arvensis du sempervirens. Les feuilles du dernier sont luisantes, persistantes, toujours vertes, à peu de distance les unes des autres; celles du premier sont opaques, glauques en dessous, caduques, et ne couvrant pas bien les rameaux. Les bractées de l'arvensis sont courtes et droites, et les fleurs solitaires ; celles du sempervirens sont réfléchies, avec une pointe étroite, un peu luisantes et les fleurs sont en bouquets. Ie dernier produit dans ses ramifications une callosité qui prend racine dans de certaines circonstances farorables, et le premier jamais.

Le montana de Villars est une plante excessivement obscure; un auteur le décrit avec les styles de l'arvensis, et sa description répond assez bien aux échantillons ràpportés des montagnes de la Suisse par M. Hooker, excepté que le fruit n'est pas hispide. Si cependant le montana de Villars et de Suter n'est pas différent de l'arvensis, il y a peu de raison de supposer que ce que les autres botanistes ont pris pour lui soit de même ; les échantillons de Schleicher, que j'ai eu occasion d'examiner, autant qu'on peut le faire de matériaux aussi imparfaits, paraissent être le rubiginosa; et comme S. J. Smith donne beaucoup de confiance il cette autorité, il n'est pas impossible que la plante d'après laquelle la description de l'Encyclopédie de liees a été faite, ne soit la même. Les détails donnés au $R$. montana dans le supplément de la Flore française, ressemblent beaucoup à ceux du rubiginosa dont nous parlons.

La variétè \% m'a paru long-temps devoir être considérée comme une espèce distincte: sous le point de vue de son port habituel elle pourrait être regarlée comme une hybride tenant du provincialis et de l'arvensis (1); car, à cause de ses fleurs, de ses feuilles aiguillonnées, et de son mode de croissance, elle semble se partager entre l'un et l'autre. Mais après avoir vu la varićté Monsonice de la dernière espèce, j'ui été convaincu que la plante dont il est question, avait le même rapport avec l'arvensis, que l'espèce sous laquelle le $M$ onsonice est placé; je l'ai rapporté en conséquence à l'arvensis; mais il est essentiel d'énoucer ce qui le distingue : ses branches portent quelques soies mêlies avec les aiguillons; les folioles, plus grandes, sont ovales-oblongues; les plus jeunes teintes de rouge; les fleurs en bouquets, très-grandes, semi-

(1) "M. Lindley n'ayant pas adopté le provincialis comme espèce distincte, je pense que c'est celui de Smith qu'il veut désigner ici. » 
doubles, de la couleur de chair la plus tendre; les styles longi, saillans, mais uon unis. Elle a été trouvée par Rau, dans les environs de Wurtzbourg.

La réunion des styles a été remarquée depuis long-temps, dans l'arvensis, par Lachenal, et adoptée par Haller et Villars. Cette particularité fut long-temps négligée, et ce n'est que depuis quelques années qu'elle a fixé l'attention. M. Decandolle fut le premier qui considéra ce caractère comme le moyen de foriner une famille ou série naturelle, parmi les roses, dus son IIortus monspeliensis, oì il détermine six espèces, dont la dernière doit être exclue; j'en ai quatre à y ajouter : le $R$. setigera de l'Aınérique septentrionale a le même caractère, mais il appartient, à cause de ses stipules subulées, à ına dernière fanille dite des banksiance.

Nota. \& II. Sabine culive un $R$. arvensis, à grandes fleurs doubles; e'est peut-être celui que J. Bauhin. Jesigne aiısi : R. silvestris; fol.glabris; $l$. pleno, albo. Hisl. 2, 任, 1. II a quelques rapports avec la variété $\gamma$ hybride de Lindley.

\section{ESPECE ROSA ABYSSINICA.}

R. Surculis scandentibus; aculeis confertissimis, falcatis; foliolis ovatis, sempervirentibus; calycibus pedunculisque toun sntosis. Lixdo. Mon. p. ri6. c. fig. - Pronv. Somm. p. 4r.

R. Abyssinica. Liows. in Salt's Abyssin. App. laiv. Linde.

Se trouve en Abyssinie (jaLt). ( $V . s$. sp. in Ilerb. Banks et Lamb).

Voici une plante d'un trés-petit nombre de roses de l'Afrique. M. Brown l'a signalíe le premier comme une espèce distincte, dans son appendix aux Voyages en Abyssinie par II. Salt qui en a lait la découverte; on ne peut la eonfondre qu'avee le sempervirens ront elle differe parliculierement par ce qui suit: ses folioles sont plus courtes, a vec uII petit support plus large ver's la pointe que vers la base; ses pélioles sont excessirement scabres, arec des glandes et des suies inégales; les calices et les pédoncules sont couverts d'un duvet épais; les aiguillons sont forts et trèz-nombrux.

\section{3․ ESPÈCE. - ROSA SEMPERVIRENS.}

F. Surculis scandentibus; aculeis subæqualibus, falcatis; foliis sem. pervirentibus. Linde. Mon. p. 117.-Pkonv. Somm. p. 42. 
R. Sempervirens Jingermaumi. Clos. Hist, 2. - Dill. Eltl. 326 . t. 214. fig. 318 .

R. Semperivens. Livis. Sp. 705. - Mal. Dict. n. 9. - Wilid. Sp. 2. 1072 - - Lawk. Ros. t. 45. - Pers. Syn. 2. 49- D.C. FI. fr. 4.416. - Arr. Kew. 3. 263. - D.C. Monsp. 133. - Sмпт. in Rees in 1. - Ken. Bot. reg. t. 459.-Prosv. Nomencl. p. 89 .

i. Scandens. MiLı. Dict. n. S.

R. Balearica. Desf. Cat. hort. par. - Pers. Syn. 2. 49.

R. Atrovirens. Viv. Fl. ital. 4. t. $G$.

R. Capreolata. Neıl. in Edimb. philos. journ. 3. 104.

Var. B. Micropny lia. Foliolis suborbiculatis.

R. Microphyla. Desf. Atl. I. 401.

Var. $\gamma$. Prostrata. Surculis prostratis. Prowv. Somm. p 42.

R. Prostrata. Lind. Mon. p. 118 . - D.C. Hort. Mons1. 138. Suppl. 536.

Se trouve dans Ja France méridionale (Decasdoule); en Portugal (Drotero); en Italie, commune à Prstum (VIviani); aux îles Baléares (Requien); en Gréce (Sibthorp); var. $\beta$ prés de Tuuis (DesforTAINes); var. $\gamma$ France méridionale (Degandolee. ( $/$. v. cult. et s. sp.)

Plante grimpante, avec des rameaux très-longs, vertclair, faibles, très-divisés, rougreâtres d'un côté, armés d'aiguillons grêles, quelque peu crochus et rouges. Fcuilles ordinairement réfléchies, très-luisantes, toujours vertes, sans aucune pubescence. Stipulcs étroites, rouges, réfléchies sur les bords. Pétioles armés de petits aiguillons crochus. Folioles 5-7, ovales, ou ovales-lancéolées, plates, simplement dentées, vert-clair des leux côtés, mais plus pâles en dessous. Fleurs très-nombreuses, blanches, odorantes. Bractées nues, !ancéolées, réfléchies, tachées de rouge. Pédoncules nus et glanduleux. Tube du calice nu ou glanduleux. Sépales cadu. ques, ovales-aiguës, presque simples, plus courtes que les pétales, scabres et glanduleuses.Pétalcs obcordés, convexes. Etamines 158-140, qui tombent bientôt. Disque conique, trèsépais. Ovaires 5o. Styles réunis en une longue colonne velue. Fruit rond, petit, couleur orange.

Plante d'agrément qui garnit en peu d'années un treillage ou un mur d'espalier, au pied desquels on la place; ses rameaux étalés et ses styles velus la distinguent du prostrata. I.e $R$. atro-virens de Viviani est décrit avec un fruit hérissé, et figuré avec un fruit glabre.

La rose d'Ayrshire décrite par M. Neill dans le Journal philosophique d'Édimbourg sous le nom de $R$. capreolata, ne paraît pas différer de celle-ci, qui n'est pas indigène d'Amérique, mais de l'Europe méridionale ou des côtes de la Barbarie. 
Nota. "La variété $\beta$. microphylla est décrite dans la Flore atlantique de M. Desfontaines; je ne l'ai pis encore vue cultivée. Quant au prostrata de Decandolle, la diffírence des styles glabres ne m'a point paru assez importante pour le distinguer du sempervirens; ses fenilles sont ovales-lancéolées, et il ne s'élève pas à plus de deux pieds; peut-être le prostratı de M. Sabine offre-t-il de légères différences; je persiste à croire que le R. d'Ayrshire tient plus de l'arvensis que du sempervirens; il perd ses feuilles vers le milieu de I'hiver. Si je counaissais l'origine de la lielle variété sempervirens latifolia, cultivée au Luxembourg, je l'aurais placée parni les indigènes; elle sera mentionnée dans l'appendix."

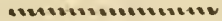 \\ 6łe. ESPÈCE. - ROSA MULTIFLORA.}

R. Ramulis, pedunculis, calycibusque iomentosis; foliolis mollibus, lanceolatis rugosis; stipulis pectinatis. Lixd. Moo. p. 119.-Prosv. Somm. p. $\dot{3}$

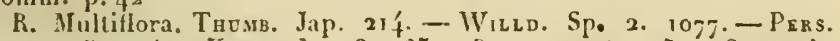

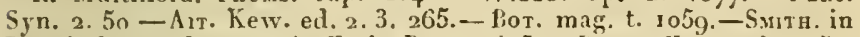
Rees in loc. - LiNdery. in Ker's. Ficg. t. 425. - Phorv. Nomenrl. 1. 57.

R. Flawa. Dons. Cant. ed.4. 121.

R. Forida. Poir. Enc. suppl. in loc.

R. Diffusa. Rnxr. Fl. ind. ined.

Se tronve au Japon (Thumberg); a la Chine (Staustov). ( $V$. v. cult. et sp. io herb. Linn. et Lambert.) Lixolex.

Arbuste de 12 a 15 pieds de hautenr. Branches Aingelliformes, nues et flexibles. Aiguillons crochus, placés par paires suus les stipules. Stipules élargies à la base, ınais pointues, adhérentes, très-dentées, cotomneuses en dessous. Pétioles très-velus. Folioles, 5 à 7 , rapprochées, ridées, lancéulées, obtuses, crénelées, d'un vert triste, el velues des deux côtés. Fieurs d'un rose pâle, nombreuses, petites, ell houquets, toujours doubles. Bractées linéilires, dentées, trèscaduques, cotonneuses, ainsi que les pédoncules. Tube du calice turbiné. Sépales ovales. Styles cotonneux 18-25, mais en colonne, plus longrs que les pétales intéricurs. Fruit turbiné, rouge-clair, non couronné par les sépales.

Nous ne connaissons cette plante dans nos jardins qu'i fleurs doubles, qui ressemblent si fort à quelques-unes de celles dı genre rubus, qu'on la connaît vulgairement sous le noun de Kose de la Chine, à fleurs de ronce. Son fruit n'a jamais été 
décrit. Je suis redevable à M. Lambert d'avoir pu l'examiner. Il en a un échantillon apporté de la Chine par sir Georges Staunton, tiré de la plante véritable, où l'on ne remarque point la pubescence des pédoncules et calices, dont les sépales sont caduques. Il est si différent des autres plantes de la même tribu, que je ne sais avec laquelle on pourrait le confondre, si ce n'est avec l'espèce suivante, dont pourtant il diffẻre essentiellement.

Le R. Grevillii, connu aussi sous le nom de R. Roxburgii, est une légère variété dı multiflora.

Nota. "J'observerai encore que la rose de Thumberg porte des fleurs blanches, et que celle de Staunton pourrait bien être une variété de la première; elle n'est connue que par la description de M. Lindley. "

\section{ISPECCE. - ROSA BRUNONII.}

R. Ramulis; foliolis lanceolatis, calycibusque tomentosis, glandulosis; stipulis integris. Lindu. Mon. p. 120. c. fig. - Pronv. Somm. p. 43.

Se trouve all Nepaul (IVAllicil, Buchavas). ( $V . s . s p$. in Herb. Banks et Lambert.) Lindeley.

Arbuste qui a l'apparence du moschata. Ses anciennes branches un peu velues, vigoureuses, armèes d'aiguillons épars, courts, forts et crochus. Les plus jeunes sont cotonneuses et glanduleuses, leurs aignillons arqués. Stipulcs linéaires, adhérentes, subulées, allongécs à leur extrémité, et glanduleuses en dehors, ainsi que les pétioles qui sont velus et garnis d'un petit nombre d'aiguillons arqués. Folioles 5- 7 , lancéolées, plates, sinplement dentées, relues sur toute la surface supérieure et vert-foncé, plus pâles et glanduleuses en dessous. Serratures très-convergentes. Fileurs en bouquets. Bractées droiles, lancéolées, velues, roulées en dedans et légèrement glanduleuses sur le revers. Pédon-. cutes velus, bruns, couverts de soies et de glandes qui sont plus épaisses sur le tube du calice, oblong et velu, mais en moindre quantité sur les sépales réfléchies. Ces dernières semblent plus longues que les pétales, et sont presque simples. Pétales blancs. Étamines et styles comme ceux du moschata.

Cette précieusc addition à la tribu des roses dont les styles sont réunis, est native du Népaul, d'oì elle a été en- 
voyée par le docteur Wallich. Elle a été trouvée par le docteur Buchanan, qui en a envoyé des íchantillons à M. Lambert. Je ne crois pas pouvoir la rapporter à aucune espece de la Flore indienne de Roxburgh, at moins que ce ne soit son $p u-$ bescens dont j'ai eu occasion de voir un dessin. Tous les euractères les plus importans du Brunonii manquent à ce dernier, et il n'en est pas plus fitit inention dans la description de Roxburghh. An reste, s'il arrivait que ce fôt la même plante, le nom vague de pubescent devrait céder à celui que j’ai préféré.

Il diffère dı moschata par ses feuilles velıes et glandılenses, ainsi que ses rameaux et son calice; les folioles sont aussi fort différentes.

"C'est avec justice que M. Lindley a dédié ce rosier au célèbre botaniste Robert Brown, auquel on doit Ia connaissance d'un grand nom're de plantes de l'Australasie. 》

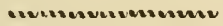 \\ 66. ESPİCE. - ROSA MOSCHATA.}

R. Ramulis nudiusculis; foliolis ellipticis, acuminatis, subtùs glancis; serraturis conniventibus; stipulis integris; sepalis compositis , acuminatis. LiNol. Mlon. p. 121. - Pronv. Somin. p. 43.

R. Moschata minor, etc. BavH. Hist. 2. 45. a 47.

Rose Mluscate. Riegn. Bot. c. ic.

R. Moschata. Nill. Dict. n. 13. - Durnt. Ilarbk. 2. 365. - Quer. Fl. Esp. 6. 205. - JACQ. Schœenbr. 3. t. $280 .-$ Wic LD. Sp. 2. $1074 .-$ Disf. AtI. 1. 400.-Latwr. loos. tt. 53-6 f.-Pers. Syn. 2. 49. Guel. Bad-Als. 2. $429-J$ AcQ. Fragm. 3r. t. 3\%.f. 3. - Ait. Kew. ed. 2. 3. 164. - DC. Cat. lorti. monsp. 135. - Sмпп. in Rees in 1. - Redout. Kos. I. 33. t. 5. 99. t. 35 -Provv. Nomencl. p. 9 1.

R Opsostemma. Eur. Meitr. $27^{2}$.

R. Glandulifera. Roxв. Fl. ind. ined.

Var. B. Nupuescera. Foliolis oblongis, acutis, impubibus; petiolis, pedicellis caly cibusque glandulosis. Livol. in l.-Provv. Somm. Idem.

Se trouve dans les champs de Tunis oil il est cultive (Desfontalnes); dans l'Espagne méridionale (Qoer, Alstroemer); à Madère (Staexton). Schuter ( $\boldsymbol{V}$. v. cult. et s. sp. Herb. Smitu, Lameert.)

Arbuste droit, très-rameux. Branches très-pei glanduleuses, armées d'aiguillons presque égaux, forts, épars et rochus. Stipules linéaires, adhérentes, divisées et subulées a lcur extrémité, frangées de glandes et velues en dessous. Pétioles velus, aiguillonnés et glanduleux. Folioles elliptiques, lancéolées, déployées simplement et finement dentées, nues 
en dessus, glauques en dessous, la nervure principale velue. Cimes très-nombreuses, d'environ 7 fleurs et davantage, corymbes arec des ramifications cotonnenses. Bractées trèscaduques, concaves, réfléchies, velues et glanduleuses. $P$ édoncules quelquefois cotonneux comme le tube glanduleux qui est ovale et les sépales réfléchies. Celles-ci allongées, un peu laciniées, tombant bientôt après les pétales. Pétales d'un blanc pur, exhalant une légère odeur de musc, presque entiers, étalés, et un peu connivens. Étamines de 80 à 85 , très-caduques. Disque élevé, épais, presque plat. Ovaires 20. Styles velus, unis en une colonne longue et gréle. Fruit petit et rouge.

Cette rose est du petit nombre de celles de l'Afrique septentrionale; on la trouve sur toute la partie de ce continent, depuis l'Égypte jusqu'à Mogador, et de là à Madere, d'où clle a été apportée par sir Georges Staunton, et par lui comınuniquée à M. Lambert. D'après le témoignage de Quer, on la trouve également dans les provinces méridionales et tempérées de l'Espagne; et dans l'herbier de Linné se trouve un échantillon d'Espagne donné par Alstroëmer. Mais l'assertion de M. 'Thory, qui la dit incligène de l'Indostan, n'est nullement fondée. Roxburg, qui la déerit sous le nom de glandutifera dans son manuscrit, n'osait décider comment elle était parvenue au Jardin de Calcutta. Il sounconne qu'elle renait de la Chine.

Elle est généralement cultivée à cause du parfum agréable et un peu musqué de ses fleurs, d'où elle tire sun nom. Toutefois nos hivers sont trol rigourenx pour cette plante. Elle prisente l'inflorescence lil plus composée du genre. Más je suis disposé à croire que la masse de fleurs qu'elle produit a été formée par l'aggrégation d'un grand numbre de rameaux très-faibles, floriféres, lesquels cousidérés séparément, ne se trouvent pas plus ramifiés qu'à l'ordinaire. Plusieurs autresespèces de roses disposées de mêne peuvent confirmer notre opinion.

Outre ces dissemblances dans l'hahitude, ce rosier diffère du sempervirens à peu près de la même manière que l'abyssinica, et ce n'est pas une observation à dédaigner que ces rosiers sont indigènes en Afrique; qu'ils ont du duvet sur leurs rameaux, des ramifications florescentes porlant un jeune fruit, caractères qui ne se retracent que sur quelques espèces de l'Asie. 
Nota. "Le Rosa nivea de M. Dupont est une plante tris-vigoureuse; ses nombreux corimbes de fleurs simples, mais tris-grandes, et d'un blanc pur, font de l'effet. P'rovenant de rraines du moschata, il conserve ses feuilles dans les hivers modérés, comme je l'ai observé cette année(1ત̇24). Ce n'est pas la première fois que je le recommande aux cultivateurs.

\section{7 $7^{\circ}$ ESPECE. - ROSA RUBIFOLIA.}

F. Ramulis impubibus; foliolis ovato-lanceolatis ; serraturis divaricatis; stipulis integris; sepalis ovatis; fructibus pisiformibus. LiNd. Mion. 1. 123. - Pronv. Somm. p. 4i.

R. Rubifolia. Lrown in $\Lambda_{\mathrm{r}}$. Kew, ed. alt. 3. $260 .-P$ unsн. $\Lambda \mathrm{m}$. sept. 1. n. 9. $-S_{31}$ ru. in Recs in 1.1

Var. $\beta$. Fexestralis. Foliolis utrinquè impubibus; floribus subsolitariis. LiNoL. fig. 15.

R. Fenestrata. Dons. Cant. ed. S. 1 go.

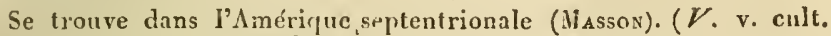
lort. SABINE. et s. sp. IIcrb. PaNKS )

Arbuste élevé de 5 ì 4 pieds ; rejetons ascendans. Brancíes vert-clair, sans duvet, ornees de quelques aignillons épars et arqués. Féuilles distantes. Stipules très-longues, étroites, nues, frangées de glandes. Pétioles nus, avec peu d'aiguillons. Folioles de 3 ì 5 , ovales, aiguës, simplement dentées. Serratures divergentes, vert-clair, mais un peu luisantes en dessus, beaucoup plus pâles et cotonneuses en dessous. Fleurs petites, rouge-pâle, environ 5 ensemble. Pédoncules et calice sans pubescence. les premiers glanduleux. Sćpales simples, ovales, velues, réléchies, caduques. Etamines caduques. Styles unis en une colonne ou massue, cotonneux. Fruit nu, de la grosseur et de la forme d'un pois.

Cette plante a toujours étémal connue, n'ayant d'autre autorité que l'Hortus Kerwensis. Dans la dernière édition, elle a été décrite par M. Brown comme une des plantes élevées de graines enroyées du nord de l'Amérique par M. Masson. C'est une espéce très-distincte qui n'a d'affinité qu'arec le moschata. Ses rameaux nus, ses pédoncules el son calice, le distinguent aisément ile celui-ci sans avoir recours à d'antres caractères. Son habitude est la même, mais ses proportions moindres. De plıs, les fleur's, qui sont rouge-pâle et petites, se distinguent tout-it-fait de celles du moschata. 
La variété $\beta$ fenestralis diffère du vrai rubifotia par l'absence totale de pubescence sur les feuilles, jar leur couleur pâle et leur texture plus mince. Une figure a été dessinèe d'après un échantillon extrêmement faible, et il ne présente pas la moindre apparence de la plante en question. Les fleurs sont ordinairement réunies au nombre de 3 à 4 ensemble.

Nota. "Je ne pourais affirıner si c'est le véritable rubifotia ou la varieté fenestratis que je cultive, cette plante n'ayant pas encore fleuri."

\section{$\mathrm{XI}^{\circ}$. TRIBU. - BANKSIANA.}

Stipula subliberæ, subula!æ, angustissimæ, sæpiùs deciduæ. Foliola sæpiùs ternata, nitida. Caules scandentes.

Obs. I.es espèces qui constituent cette dernière tribu sont remarquables par leurs longs rejets élégamment courbés pour la plupart, et couverts de fleurs blanches; les fleurs sont ternées et luisantes. Le caractère distinctif de cette tribu est dans les stipules caduques, subulées et très-ćtroites. Les fruits varient beaucoup. Le $R$. hystrix a ses rameaux soyeus, et le setigera a ses styles ríunis.

\section{ESPËCE. - ROSA LAVIGATA.}

R. Stipulis lineari-lanceolatis, semiadnatis; petiolis inermibus; fructibus inuricalis. Linde. Mlon. P. 125. - Provv. Somm. p. 45.

R. Lavigala. Mich Bor. Am. 1. 295. - Pers. Syn. 2. 49. - Pursu. An sept. 1. n. 10.-Smitr. in Rees in 1. - Posv. Nomencl. p. S9.

Se trouve dans les bois ombragés de la Géorgie (Porsn). Fraser. ( $I$. s. sp. Herb Sabine, Lindu. Herb. Nichavx. II. R.. N.)

Tige grimpante (Jvrsn.). Aiguillons épars, arqués. Stipules très-étroites, attachées aux pétioles par leur partie infërieure, non caduques en apparence, et frangées de glandes. Pétioles nus. Folioles 3 , ovales, lancéolées; les anciennes sont coriaces et luisantes, simplement dentées, sans aucune pubescence. Fleurs solitaires, grandes, hlanches. Pédoncules et tube du ealice couverts de soies trés-denses, faibles et inégales. Sépales ovales, étalées avec une pointe, entières, dilatées au sommet avec quelques soies sur les bords. Pétates dépassant ceux-ci, presque entiers. Étamines nombreuses: 


\section{5}

masse de stigmates grosse et cotonneuse. Disque épais. Fruit oblong, rouge, muriqué ou armé d'aiguillons raides et couronné par les sépales endurcies.

Native des bois de la Géorgie, où l'on dit qu'elle grimpe autour des arbres les plus élevís. Sa ressemblance a vec l'espéce suivante est très-grande, et a fait prendre par erreur dans unc circonstance la plante chinoise pour l'américaine, ce qui lui donne le nom de cherokensis. Il est pourtant facile de les distinguer à l'aide des caractères suivans. Le lavigata a une tige grimpante, persistante, des stipules demi-adhérentes, des pétioles nus et des nervures aux feuilles. Le sinica a une tige trainante, des stipules subulćes et caduques, des pétioles et des nervures très-aiguillonés; mais leurs fruits sont si semblables qu'il est difficile de les distinguer.

l.e seul échantillon que j'ai vu n'a été communiqué par M. Sabine.

Nota. « J'ai examiné les échantillons de Michaux dont on voit l'herbier at musium d'histoire naturelle de Paris; mais j'ai dẹjà fait remarquer qu'on ne pourait pas en faire une description exacte, vu leur dégradation. ”

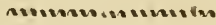 \\ $69^{\circ}$. ESPECE. - ROSA SINICA.}

R. Stipulis setaceis, deciduis; petiolis costâque aculeatis; fructibus muricatis. Lisor. Mlon. p. 126. cum fig. - Prosv. Somm. p. $\{5$.

$k$. Alba. Chersonensis; foliorun margine et rachi medid spinosis. Procks. Amalth. 185.

R. Sinica. $\Lambda_{\mathrm{IT}}$. Kew. ed. alt. 3. ${ }_{2} \mathrm{G}$.

R. Trifoliata. Bosc. Dict. fide Porr.

R. Ternata. Polr. Enc. bot. 6.284 - - Provv. Nomencl.p. 100.

R. Cherokensis. Doos. Cant. ed. 8. 172.

R. Nivea. D. C. llorti. Monsp. 137 .

( $V$. v. cult. et s. sp. Ilerb. Danks). Lindu. ( $/$. s. sp. Herb. du Dresnay. N.).

Branches traînantes, armées d'aiguillons épars, rouges, égaux et crochus. Feuiltes très-luisantes. Stipules sétacées, caduques, frangées de glandes. Pétioles non cotonneux, armés de nombreux et petits aiguillons. Folioles ternées, ovales, lancéolécs, finement dentées, très-vertes en dessus, plus pâles en dessous, avcc une côte aiguillonnée. Fleurs blanches solitaires. Sépales raides, entières. Fruit rougeorangé, muriqué, couronné par les sépales étalées et raides. 
Cette plante est assez souvent cultivée dans les pépinières, où elle n'a jamais donné de fleurs. Mais à Montpellier où elle a fleuri, M. Decandolle l'a prise pour une nouvelle espéce, et l'a publiée sous le nom de $R$. nivea. Il n'y a pas de doute que c'est la même plante donné z dans l'Hortus Kewensis pour sinica, nom que j'ai conservé. Il est bon d'observer que Linnć avait en vue une autre plante dans son spécimen, comme je l'ai déjil fait voir dans mes remarques sur le $R$. indica.

J'ai déjà fait observer à l'article précédent les différences entre cette plante et le lavigata. Leurs fruits ont tant de rapports qu'il est bien difficile de les distinguer. Le fruit du sinica, cueilli près de Macao où il est très-commun, m'a été donné par M. Sabine, et celui du lavigata par M. Fraser. La planche 16 est copiéęd'après un dessin chinois que possède S. Jos. Banks.

Nota. J'ai observé l'échantillon de Il. du Dremay, recueilli dans le jardin royal de Caserte, près de Naples. I, fruit est assez gros et entièrement couvert ainsi que le pédoncule d'aiguillons on de soies très-raides, roussâtres, qui m'ont rappelé ceux du robinia hispida.

“Je passe le $R$. recurva jusqu'à ce qu'il soit plus connu; il n'est cité par Lindleỹ que d'après le manuscrit de Roxburgh.

\section{ESPECE. - ROSA SETIGERA.}

R. Sepalis pennatifido-setigeris; stylis coafitis; fructibus muricatis. Lindu. Mon. p. 128. - Pronv. Somm. p. 45.

R. Seligera. Mich. Dor. $M$ m. 1. 295. - Pers. Syn. 2. 4S.-Pursh. Am. sept. 1. n. 7. - Sмлтн. in Rees in loco. - Puln. Dict. p. 295.

Se trouve dans I'Amérique septentrionale (Mchaux). ( $V$.s. sp, in Jlerb. Mich. H. R. N.)

Arbuste droit, divisé en rameanx glabres, cylindriques, armés de deux aiguillons stipnlaires à la base des feuilles et de quelques autres épars le long des branches. Stipules subulées. Pétioles nus, recouverts de soies et de petits aiguillons. Folioles 3 , rarement 5 , ovales et ordinairement pointues, glabres, à serratures aiğuës. Flcurs nombreuses, quelquefois solitaires, rouges. Pédoncules longs, glabres, munis de soies. Tube du calice rond et glabre. Sitpules très-étroites avec une pointe aiguë, souvent pinnatifides, cotonneuses ct couvertes de soies en leurs bords comme les barbes d'unc. 
plume. Pélates larges, obcordés, Slyles deux fois aussi longs que le tubeducalice, et réunis en une colonne glabre. Fruit glohuleux, oglabre. (Tiré du manuscrit de M. Achille Richard.)

Si l'espece précédente est très-peu connue (recurva), je snis an moins redevable a $\mathbf{H}$. Achille kichard, qui a pris la peine d'examiner pour mon compte l'Herbier de Michaux, et qui m'a fourni la description que je présente ici. La réunion des styles dans le setigera doit le distinguer du reste de la tribu.

Il est encore ì examiner si la variété $\beta$ eitée par Michaux dans sa Flore Boréale, ne serait pas une espéce distincte. Les plantes ont été trouvées dans la Caroline-Inférieure. (Poiret.)

\section{7}

R. Armis ramulorum confertis; majoribus falcatis; foliolis ovalis; fructibus hispido-muricatis. Lind. Mín. p, i 29. cum fig. - Provv. Somm. p. 45 .

Setrouve en Chine, province de Kung-Si. (STauvroy), et au Japon (Herb. Lamb.) ( $V$. s. sp. Herb. Banks et Lambert).

Branches vertes, fiagelliformes, armées de nombreux aiguiltons raides et très - petits, inégaux et droits aver; quelques-uns plus grands et arqués. Miris les premiers sont extrêmement petits sur les vieilles tiges. Feuilles séparées. Stipules très-étroites, adhérentes par leur moitié. La partie libre caduque et laissant par son absence une longue escarre. Pétioles sans duvet avec quelques aiguillons crochus. Fotioles 3 ensemble, ovales, plates, luisantes, a scrralures sunples, pédicellées, vert-foncé en dessus, pâles en dessous arec une côte aiguillonnée. Bractíes nulles. Pédoncules à fruit oblong, pourpre, avec des soies et des aimuillons raides très-droits. Sépales persistantes, raides, convergentes, ovales, pointues; presque entières avec quelques aiguillons grêles et raides, dont quelques-uns sont à la marge. Disque plat, charnu. Styles velus, renfermés. Fleurs grandes.

Je n'ai été à portée que d'examiner deux échantillons de cette espèce rare: l'un avec du fruit, qui a servi de modèle á la Ggure, est tiré du Muséum de sir Jos. Banks; l'antre en fleur, mais dans un état très-imparfait, dans l'Herbier de M. Lambert, qui l'a obtemu atvec un grand nombre de plan- 
tes du Japon, provenant, dit-on, de la prise d'un bâtiment hollandais dans le cours de la dernière guerre. Les branches sont couvertes de sojes petites, courtes et raides et entremêlées de quelques aiguillons plus forts et arqués. Il paraît par l'étiquette de l'échantillon de $\mathrm{H}$. Lambert que le collecteur a. pris cette plante pour le canina de Thumberg.

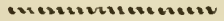 \\ 72. ESPËCE. - ROSA MICROCARPA.}

R. Floribus corymbosis; fructibus pisiformibus, inermibus. LINOL. Mon. p. 13o. cum fig. - Provv. Somm. p. 45.

R. Cheusana glabra, Juniperi fructu. РЕт. Gaz. 57. 1. 35. f. I.

R. Indica. Lisv. $\mathrm{Sp}$. pl. p ;05.

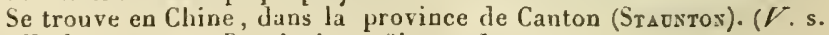
sp. Herb. Banks, et Pict in icon. Sinens. Linda.)

Branches flagelliformes, grêles, défendues par quelques $a i$ guillons petits, épars, caducs et crochus, souvent un peu cotonneux. Feuilles distantes, caduques. Stipules subulées, se détachant bientôt. Pétioles cotonneux et nus. Fotioles 3-5, oblongues ou ovales, lancéolées, nues, simplement créneliés ou dentées, luisantes et vert-foncé en dessus, plus pâles en dessous. Fleurs trés-nombreuses, petites, blanches. Bractées caduques, supports glabres. Fruit écarlate, de la grosseur et de la forme de celui du Cratagus oxyacaniha. Styles 15, velus, très-peu saillans. Sépales caduques. Disque plat. Péricarpe $2-5$, arrondi, nu, très-luisant.

Il ne peut y avoir une preuve plus frappante de la connaissance imparfaite que Linné avait des roses asiatiques, qu'en le voyant citer celle-ci bien figurée par Petiver comme étant l'indica. Willdenow a reproduit cette erreur, parce que probablement il regardait le $R$. indica de Linné comme une plante qui lui était inconnue.

Il a beaucoup d'affinité avec le banksice, dont sa tige garnie d'aiguillons, très-cotonneuse étant jeune, et ses folioles d'une forme différente, le distinguent suffisamment.

\section{7\%". ET DERNIERE ESPECE. - ROSA BANKSIAE.}

R. Ramis et fructibus inermibus. Linel. Mon. I3 I. - Pronv. Summ. 1). 46.

K. Banksia. Lrows. in Arr. Kcw. ed. alt. 3. p. 258. - Smitr. in 
Rees in 1. - Curt. Mag. t. Ig54. - Lisnz. in Ken's Reg. 1. 39-. - Pisnout. Ros. - Puir. Enc. Suppl. p. $; 16$.

R. Bankiana. Abel. Clin. 160.

R. Inermis. Roxв. MS.

Se trouve en Chine (KER). ( $V$. v. cult.)

Branches sans armures, fuibles, grimpantes, vert-loncé. Stipules subulées, quelquefois velues, tombant bientôt. Pétioles nus, rarement velus. Folioles 1-5, plates, ublongues, - lancéolées, obluses, souvent ondulées, simplement dentées, sans pubescence, excepté à la base de la nervure dorsale, où il y a des poils. Fleurs penchées, nombreuses, petites, trèsdoubles, d'une odeur faible, mais tris-suave (elles sentent la violette). Bractées monues, bientôt caduques. Péloncules nus, très-grêles, un peu épaissis à la partie supéricure. Tube dı calice hémisphérique. Sépales ovales, pointues, entières. Styles séparés, un peu saillans. Fruit inconnu.

Cette plante est une des plus élégantes du grenre, d'une végétation vigoureuse en plein air, et produisant ses charmantes fleurs avec la plus grande profusion. M. Brown est le premier qui en ait parlé dans la dernière édition de l'Hortus liezvensis, et il en lail hommage à lady Banks; on en troure une excellente figure dans le Botanieal Register.

Le $R$. inermis de Roxburghl, dans sa Flora indiea, qui n'a pas encore paru, est probablement cette espéce; et s'il en est ainsi, une variété appelée $W$ ong-mouc-henong a fleurs jaunes doubles, se cultive dans le jardin botanique de Calcutta.

"Le rosier de Banks est une acquisition d'autant plus intéressante pour les amateurs de jardins qu'il paraît devoir s'acclimater aux environs de Paris, et sous ce rapport il est moins délicat que le multiflora. 11 a fleuri en pleine terre le printemps dernier $(1825)$, dans les pépinières de H. Noiselle.

„-Ici se termine cette longue série d'espèces, et l'on me fera peut-être le reproche d'en avoir augmenté le nombre, si l'on compare cet ourrage avec les derniers qui ont paru sur ce beau genre. Mais si j'arais osé admettre toutes celles que nous présentent les prodromes et les flores particulieres, le nombre en eât été doublé. Au lieu d'augrmenter, je me suis permis de nombreuses éliminations; mais, pour compléter en quelque sorte le travail du botaniste anglais dont je donne la traduction, je dois mentionner ici non-seulement les plan- 
tes qu'il n'a pu placer parmi ses familles naturelles, mais encore celles qui lui semblent douteuses et paraissent se rapporter à d'autres espèces déjà connues. "

\section{ESP'ECE de LINDLEY.-ROSA PSEUD'INDICA.}

Se trouve cn Chine. (8. Ріст.) Dibl. Lambert.

exweencheresvest

28. ESPËCE, idem. - ROSA X XNTHINA.

Se trouve en Chine (V. Ріст.) Bibl. Lambert.

\section{ESPÈCES DOUTEUSES}

\section{Qui ont beaucoup de rapports avec les pre- mières.}

$79^{\mathrm{e}}$. ROSA AGRESTIS. (GMEL. Bad.-Als. 2. 416.)

R. Germinibus subglobosis, pedunculisque hispidulis; foliolis rotondis, obtusis, æqualiter dentatis, subtùs venosis, albiclo-tomentosis ; caulc aculcolis sparsis, rectis; floribus solitariis. GMEL.

Hab. in agris argillaceo-lutosis, calcarcis, apricis, inter segetes nec alibi. Gmes.

Arbuste droit, d'un pied à un pied et demi. Branches menues, glabres, vertes, sans armure à la base. La partie supéricure couverte d'aiguillons, mais épars, petits, inégaux et droits. Folioles sessiles, rondes, obluses, égalemenıdentées, glabres et d'un vert foncé à leur surface, veinées et couvertes l'un duvet blanchâtre en dessous.Pétioles presque glabres.Stipules étroites, lancéolées, glabres et entiéres. Fleurs solitaires, grêles, blanches. Tube du calice scabre, arrondi. $S \dot{-}-$ pales composées, hispides, bordées de duvet cotonneux et blanchâtre, plus courtes que les pétales. Fruit arrondi, glabre, rouge-fauve. Gmes.

Peut-être ayant des rapports avec le tomentosa. 


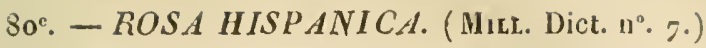

R. Foliis utrinquè villosis; calycis foliolis acutè serratis; fructu onl.1bro. Misl.

IIab. in llispanit.

Tige de quatre pieds de haut. Aiguillons forts. Ftcurs rouge-clair, paraissant au mois de mai. Mituer.

\section{1 ${ }^{\mathrm{e}}$.-ROSA GEMELLA. (Wirld. Enum. 544.)}

R. Germinibus depresso-globosis, pedunculisque glabris; floribus subgeminatis; foliis oblongis, acutis; petiolis venisque subtus pubescentibus; aculeis caulinis geminatis.

Hab. in Anericî boreali.

Aculei breves, unciuati, geminati, infrà axillares. Non stipulares. Petala rubra. Media inter R. Lucidam et Carolinam, sed folia nullo modo nitida. WILLD.

Ceci est adopté par Pursh sans autre addition. On remarque que c'est un arbuste nain, portant de grandes fleurs, qu'on voit dans les montagnes arides depuis la NourelleAngleterie jusqu'à la Caroline.

le gemella peut être une espèce distincte; mais, d'aprìs les détails précédens, il ne se distingue du carolina que par sun fruit glabre.

Le lieu natal du spécimen de Linné, décrit par S. James Smith dans l'Encyclopédie de Rees, est inconnu; ils sont tresincomplets; mais, autant que l'on peut s'en former une opinion, ils sont européens et appartiemnent au cinnamomer, les folioles n'étant qu'un peu plus grandes qu’à l'ordinatire. Ils ne répondent cerlainement pas à la description de Willdenow., inter tucidam et carolinam. "Je soupconne furt qu'un rosier que j'ai reģ de $\mathbf{M}$. Noisette, et qui veuait de Dupont comme carolina, est le genella de Wiruo. J'en formerai une variété. Voyez Rosa carolina."

$$
\mathrm{S}_{2} \text { e. - h. LYONII. (Pursh. An. sept. 1. 575.) }
$$

R. Germinibus subglobosis, slabriusculis; pedunculis hispirlis; prtiolis subaculeatis; caule glabro; aculeis sparsis, rectis; foliclis $3-5$, 
ovato-oblongis, acutis, serratis, suprà glabriusculis, subtùs tomentosis, superioribus simplicibus; floribus subternatis; stipulis linearibus; calycis laciniis tomentosis; linearibus, vix lacinianis. PORSH. loc. cit.

Se trouve ì Ténessée (Lrov).

Fleurs rouge-pâle. Feuilles petites, avec des nervures colorées, décrites par Pursh d'après des échantillons de l'herbier de H. Lyon.

Voici encore une autre plante qui a des rapports évidens avec le carolina, quoique peut-être assez distincte à raison de ses aiguillons épars. Miịs quand M. Pursh eut vu les roses de M. Sabine à llimms, il en distingua une qu'il dit être le Lyonii. Je n'ai pas été assez heureux pour la voir avec ses feuilles; nais dans cet état elle ne diflére en rien du carolina si ce n'est par son fruit glabre et quelques aiguillons arqués.

Nota. J'ai vu aussi dans la collection de M. Noisette un rosier qui répond assez au Lyonii, inais il n'avait pas encore fleuri.

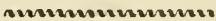

83e. R. POLLINARIA. (SPReNg. Plant. min. cogn. pag. 2, p. 66. )

R. Calycum tubis ovatis, pedunculisque hispido-glandulosis; petiolis aculeato-glandulosis; foliolis ovato-subrotundis, utrinqué glabris, serratis; deutibus glanduloso-serrulatis ; trunco aculeato. PoLLEN. Plant. veron, $\mathrm{r}$ 3. ex Polret.

Cette espèce, qui a des fleurs blanches, a de l'affinité avec le sempervirens; ses feuilles sont simplement dentées, les pétioles glabres et les divisions du calice entières. Elle a une tige de 4 à 6 pieds de haut, couverte d'aiguillons crochus. Les rameaux sont hispides, rougeâtres, paniculés et portent 3 fleurs au plus. Pétioles soyeux et glanduleux. Folioles 5-5, ovales, arrondies, quelquefois obtuses, vertes, luisantes on dessus, plus pâtes en dessous. Les serratures glinduleases. Stipules cilices. Sépales pinnatifides. Fleurs grandes, pourpres. Pétales nvales, arrondis, légèrement odorans. Tubes ovales, hispides. Styles séparés, deux fois aussi courts que les étamines. Fruit ovale, globulcux. Il crô̂t dans les haies au pied du mont Ballo. Polun ex Poiret.

C'est une pure variété du rubiginosa. Pollin a eu sans doute le dessein le la comparer avec le sempervirens de quelques botanistes allemands, mais non de Linné.

Nota. Le mont Baldo est un licu cher aux botanistes; il est 
situé près de Vérone. Plusieurs plantes qui lui sont propres, un carex par exemple, ont gardé son nom.

\section{n.................... \\ 84c. - R. HISPIDA. (Poir. Enc. bot. n. 15.)}

R. Germinibus globosis pelunculisque hispido-aculeatis; foliolis ovatis, subiùs albido-tomentosis; caule aculeis sparsis; floribus solitariis. Polr. l. c.

A l'occasion de celle-ci, M. Poiret cite le $R$. pomo spinoso, folio hirsuto de J. Bauh. Hist. 2. 38. avec quelques doutes. Cette figure semble être le $R$. villosa, et j'aurais été tenté de dire aussi l'hispide. Mais on le décrit avec des feuilles glabres, ce qui ne peut appartenir au villosa. Il est possible que ce soit une variété dutomentosa, mais dans ce cas la synonymie de Bauhin est citée mal ì propos. Nota. Le rosier que j'avais pris pour l'hispida de Poiret appartient évidemment anspinosis. sima de Lindley. Cette variété est devenuê très-rare, et l'on ne peut recourir aujardin Royal de Paris où elle ne se trouve plus.

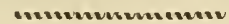 \\ 85e. - R. EVRATINA. (Bosc. Dict.)}

R. Germinibus ovatis, hispidissinis ; ramis petiolisque subinermibus; foliolis quinatis ternatisve; pedunculis hispidis, fasciculatosubumbellatis, terminalibus. Pork. Enc. suppl. 714 .

Se trouve en Caroline. Poik.

Cette espèce a de l'affinité avec le multiflora et plus encore a rec $\ell^{\prime} a l b a$ par la forme de ses feuilles. Ses tiges et ses branches sont glabres, ordinairement inermes ainsi que les pétioles. Les feuilles sont composées de 5 et quelquefois de 5 folioles assez grandes, ovales, obtuses, presque également dentées, vertes en dessus, plus pîles et quelquefois glauques en dessous. Stipules entières avec deux dents aiguës. Les fleurs sont ordinairement terminales, en bouquet presqu'en ombelle. Pédoncules droits, uniflores, très-soyeux et glanduleux ainsi que le tube du calice qui est ovale, et aussi ses divisions, qui sont ovales, entières, arquées et terminées par une longue pointe. Les fleurs sont moyennes et d'un rouge pâle.

Cette plante croît dans la Caroline; elle est cultivée dans la plupart des jardins de l'Europe. (Poır.)

Si on ne l'avait pas comparée au multiflora et à l'alba, je 
l'aurais prise pour quelque variété presque inerme du carolina, qui varie prodigieusement scus les rapports de ses proportions, de la forme de ses feuilles, de ses aiguillons et de la pubescence. ( IINDLEY.)

" $N$. Je ne puis être de cet avis; il y a toute apparence que l'evratina n'est pas une plante américaine, mais qu'elle a pu être apportée aux États-Unis comme plusieurs autres (l'A $\mathrm{yr}$ shire rose). Elle a plus de rapports avec l'alba qu'arec toute autre, et sa nombreuse inflorescence la rapproche du moschata, ce cruil'a fait nommer par les jardiniers muscade rouge des Hollandais; mais je persiste à la considérer comme espèce bien distincte. "

86". - R. REDUTEA GLAUCA. (Trony. Ired. Roses. I. p. 101 . t. 38.)

Celui-ci, comme II. Thory le remarque, ressemble ì une production hybride entre le rubrifolia et le spinosissima, ayant la couleur du premier et quelque chose les habitudes dusecond.'Toutefois, ces deux variétés remar(quables du systyla et de l'arvensis que j'ai décrites, me font pencher à la rapporter au rubrifolia dont, en réalité, il ne différe poirt, excepté qu'il est plus petit et qu'ila moins de soies. Les aiguillons à la base du rejet, dans la figure de Redouté, sont très-semublables a ceux du rubrifolia. (Lisd)

"Nola. Il y a un si grand contraste entre les caractères des pimpinellifolice et des canince, que je suis surpris que M. Lindley ait ici donné la préférence au rubrifolia. Je cultive aussi le reduteana, dans lequel je remarque les caractères principaux du spinosissima; et parmi le grand nombre de R. pimpinella que je possèle, plusieurs ont le feuillage presque aussi glauque que celui-ci, notamment une variété que j'ai observéc à Malmaison. »

8; . - R. CLINOPHYLLA. (Thory in Pied. lios. 1.43. t. 10, )

Tige en buisson, soyeuse avec des poils. Branches grêles, velues. Aiguillons stipulaires, géminés. Feuilles penchées. Fotiotes oblongues, elliptiques, doublement dentées, lui- 
santes à la surface, velues au revers. Pétioles glanduleux, velus, quelquefois aiguillonnés. Stipules étroites, frangées, aiguës. Flcurs solitaires. Pédoncules très-courts, velus. Tubc du calice arrondi, velı, quelquefois garni de feuilles florales. Sépales indivisées, aiguës, soyeuses. Pétales blanes, un peu cordiformes, jaunâtres à la basc. Fruit arrondi. Tı̊nY, loc. cit.

Je ne puis en juger que par le dessin de Redouté et la description de Thory. Il appartient certainement à la tribu des bracteate, et je ne balancerais pas de le rapporter àl'involucrata, si je n'avais l'autorité de M. Sabine pour établir leur différence. Je ne vois pas dans le dessin en quoi il s'en éloigne, si ce n'est dans l'absence des bractées dans le clinophylla, et comme on n'en parle pas dans la description, je dois penser qu'elles n'existent point. ( Linde.)

"Nota. Je sais bien que H. Thory ne parle pas des bractées, mais il indique des feuilles florales à la base du calice, et il place cette plante dans son groupe 25 des bractéatées. Il rapporte comme synonyme, mais avec un point de donte, l'iuvolucrata de Doon Hort. Cantabr. Reste à savoir si ce deruier est le même que celui de Roxburgh."

SS - - R. TRI PHYLLA. (Roxв. Fl. ind. ined.)

Grimpant et armé. Feuilles ternées: folioles lancéolées.

Plante apportée de la Chino au jardin de Calcutta, où elle croît vigoureusement et s'étale beaucoup. Elle est connuc daus les jardins des cultivateurs chinois sous le nom de Tshateboy-fa. Roxburgh n'en a point envoyé de figure. Il est possible que ce soit le microcarpa, et je ne connais aucune es. péce de li Chiue à laquetle on puisse appliquer lis deseription précédente.

"Nola. Je forme le vou que ce rosier, lorsqu'il sera assez connu pour en former une espéce distincte, comprise dans la tribu des banksiance, soit dédiéà M. Redouté, dont lesconnaissanees et le beau talent méritent bien qu'une belle espéce orientale paraisse sous son nom. 》

$$
89^{\circ} \text { - R. CINNAMOMEA. (Lour. Coch. 333.) }
$$

Iloa-Koê. Cochinchinens, - Mui-Hoâ. Sinens. 


\section{6}

Se trouve indigène et cultive à la Cochinchine et à la Chine: LOUREIRO.

Tige en buisson, touffue, de 5 pieds de haut. Branches aiguillonées de même que les pétioles. Fleur's très-rouges, solitaires. Tube du calice rond. Pédoncules inerınes. P'resque point d'odeur. Lour.

\section{0. - SPINOSISSIMA. (LOUR. Coch. 325.)}

Hoa-llùng-Tau. Cochinchinens.

Se trouve également en Cochiuchine (Lodreıro).

Tige en arhuste de 6 pieds de haut, un peu grimpante, trisaiguillonnée. Fleurs carnées, sans odeur. Tube du calice arrondi. glabre. Pélioles et pédoncules aiguillonnés. Peutêtre le sinica. Lour.

Il est très-évident, parla description ci-dessus, que la plante de Loureiro n'est pas bien nommice, et il n'y a aucune plante chinoise à laquelle on puisse la rapporter.

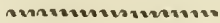 \\ 91. - R. ADENOPHYLLA. (WILld. Exam. 526.)}

F. Germinibus ovatis; calycibus pedunculisque glanduloso-hispidis; petiolis glanduloso-pubescentibus, incrnibus; foliolis simpliciter serralis, subtis glaucis, margine glandulosis; aculeis ramorum sparsis. Willaenow. loc. cit.

Se trouve

Flos magnus ruber, petalis emarginatis. Hæc flore simplici est. $A$ duabus procedentibus (R. turbinata et pulchella) fisuâ germinis, joliis rigidioribus minutè simpliciter serratis diver'sa. WILlo.

Peut être alliée au parvifolia, siol'on peut l'en distinguer. Mais on $n^{3}$ a encore aucune connaissance de cette plante, si ce n'est par un échantillon.

\section{$9^{2^{\mathrm{e}}}$. - R. TUGURIORUM. (Willd. Enum. 544.)}

P. Germinibus subrotondis, glabris; calycibus pilosis; pedunculis hispidis; petiolis villosis, aculeatis; caule aculeis sparsis. WıLb. l. c.

Habitat.....

Specics ad eatruendum casas $\dot{v}$. tuguria aptissima : in vernaculà linguâ Tapeten rose audit. WiLL. 1. c. 
J'aurais deviné que c'est l'arvensis; mais il n'est rien dit de ses styles, et Willdenow ne voudrait pas sans doute décrire deux fois le même individu.

\section{$9^{5 \text { e. }}$-R. PULCHELLA. (Willd. Enum. 545.)}

R. Germinibus subrotondo-oboratis; pedunculis calycibusque glanduloso-hıspidis; petiolis glanduloso-pubescentibus, inermibus; aculeis caulinis sparsis. Willoenow. loc. eit.

Habitat....

Affinis pracedenti (R. turbinala), sed caulis triplo minor ; flores parvi; germinis forma diversa, petioli non aculeati et falcata subrotunda qua in precedente subrotondo-ovato. WiLLD.

Est-ce la rose de Meaux de nos jardins ou quelque variété du gallica?

\section{4.}

Fruit rond. Feuilles cotonneuses en dessous, leurs bords glanduleux. Cr.

Se troure en Suisse aux environs de Bruel et de Winterthour.

Peut-être le $R$. myriacantha de Decandolle. (Clanrv.) Mais cela ne peut pas être, car cette espèce a lcs feuilles glabres des deux côtés.

\section{5 $5^{\mathrm{e}}$ - R. GLANDULOSA. (Decand. Suppl. 559.)}

Cette espèce élégante forme un buisson épais de 7 à 8 pieds de haut. Les aiguillons sont peu nombreux, droits et asse z grêles; ceux des pétioles sont petits et arqués, entremêlés de poils glanduleux. Folioles de 5 à 7 , très-glabres, un peu gliuques, ovales, obtuses, petites, ì doubles serratures, avec des dents glanduleuses comme celles de l'églantier. Fleurs solitaires, rouge-clair. Pédoncules et tube du calice couverts de longues soies spiniformes et glauduleuses. Stiputes frangées de glandes. Tube ovale. Sépalés presque toujours entières, un peu glanduleuses en dessous. Cette belle rose croît dans les liaies et les halliers aux environs de Briancon, surtout au-dessous de la ville et dans la vallée qui conduit au Lantaret. Elle fleurit en juillet. Decand. I. c. 
Doit-elle être distinguće du rubiginosà ? ou est - ce une variété du tomentosa à feuilles glabres? Linde.

$$
\text { 96. }- \text { R. ARBOREA. (Pers. Syn. 2. 50.) }
$$

R. Caule arboreo; foliis pinnatis; foliolis ovatis.

Se trouve en Perse (OLIVIER).

Plantulas juveniles et seminibus apportatis tantùn modò vidi. Persoon. 1. c.

Nota. Je tiens de M. Bose que le $R$. arborea de Persoon n'est autre chose que le moschata qui parvient en Perse à une grande hauteur.

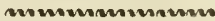

$$
\text { 97" - R. FARINOSA. (RAU. Enum. 147•) }
$$

R. Calycis tubo oviformi pedunculisque supernè glabris; foliolis ovalibus, utrinquè villosis, mollissimis, duplicato-serratis ; petiolis tomentosis cauleque aculeatis; aculeis rectiusculis. Rau. l. c.

R. Farinosa. Bechst. Forstb. p. 243. n. 159 et p. 1646.

Se trouve aux environs de Wurtzbourg. liau.

De trois à quatre pieds de haut. Aiguillons forts, droits; les rameaux armés d'aiguillons laibles, droits et quelquefois réfléchis, mais sans armure sur les extrémités. Pétioles couverts de glandes blanchâtres. Folioles blanches et douces des denx côtés, luisantes en dessus comme de la soie, et grlanduleuses à la nervure principale. Pédoncules 1-3, nus ì leurs extrémités, cotonneux à la base. Sépales composées et glanduleuses. Fleurs rouge-pâle. Fruit en toupie, rouge foncé. Rau. loc. cit.

Serait-elle une espèce distincte et très-remarquable ? ne serait-elle pas plutôt une variété naine du tomentosa, on peut-être la même que notre rose velue de Sussex, variété du R. Salini?

\section{8 $8^{\mathrm{e}}$ - R. SEMIPERVIRENS. (Rav. Enum. 120.)}

Probablement une variété du múiginosa avec des rejets rampans, des feuilles et des stipules nues. Il est étonnant qu'une plante aussi bien connue par ses fenilles persistantes et Inisantes, des styles réunis et des fleurs blanches, que 
le sempervirens, se tronve confondue arec une plante dont les feuilles sont eaduques, les styles séparés et les fleurs rouges. Lindu.

\section{9'. 一 R. TRACHYPHYLLA. (Rav. Enum. 124.)}

Elle doit sans contredit être rapportée à quelque variété du mubiginosa, dont elle diffère cependant par les serratures peu communes de ses fenilles, et ses airuillons stipulaires. Il est difficile que co soit le sepium, dont les feuilles ne sont pas arrondies à la base.

\section{0'. - R. ORBESSANEA. (Redout. Ros. 2. 21. C. fig.)}

Il paraît que ce rosier est le produit d'une plante cultivée daus les jardins et peut-être une variété du gallica, avec lequel il a des rapports par ses sépales, son labitude et même ses aiguillons; mais le fruit diffère dans sa forme, et quoique sous ce rapport il se rapproche du turbinata, il s'en éloigne tellement sous les autres que l'on ne peut en faire une seule egt mêue espèce. Lixur.

$$
\text { 101. }- \text { R. FRAXINEA. (WiLld. Enum. Suppl. 57.) }
$$

R. Germinibus ellipticis, glabris; pedunculis glanduloso-hispidis; petiolis subaculeatis, glanduloso-hispidis; foliis glabris; caule aculeis sparsis. Wılld.

Petala obcordata, saturaté rubra.

"Ce serait grossir inutilement ce volume déjábien fort que de rapporter avec détail toutes les espèces non connues ou seulement indiquées dans les eatalogues du jardin botanique de·l'île de Bourbon, dans l'Encyelopédie méthodique el autres ouvrages modernes. Depuis que M. Lindley a composé sa Monographie, le nombre en est encore augmenté. " 


\section{SPECIES NOMINE TANTUM NOTE.}

I. R. macrocarpa.

2. R. mutabilis, MEUR. AIT. 15.

3. R. lutetiana, Leman. Journ. phys, vol. 87 .

4. R. urbica. id.

5. R. rustica. id.

6. R. tomentella.

7. R. pubescens. id.

8. R. hystrix. id. n. Lindu.

9. R. nemoralis. id.

10. R. subvillosa. id.

11. R. cymbifolia.

12. R. foliosa, id.

3. R. ambigua.

14. R. poterium. $i d$.

5. K. Crlsii.

36. R. eriocarpa. id.

7. R. parvifolia. id.
18. R. ancistrum. LinnL.

19 R. neglecta. id.

20. R. balsamica. Willd. Enum. suppl. 38 .

2 I. R. apiifolia. W. 1. c.

22. R. corallina, id.

23. R. millesia. Linv. Amœn. acad. 4. 48\%.

24. R. rosenbergiana. Tnor. prodr. p. I2 1 .

25. R. malmundariensis. id.

26. R. verticillacantha. $i d$. et $\mathrm{ME}_{\mathrm{E}-}$ Rat. ed. $I$.

27. R. bibracteata. Poiret. ex Bot. fl. Andegav.

28. R. candolleana. Tror, in. Redout. Ros. 2. p. 45 . c. fig. 


\section{CATALOGUE ALPHABÉTIQUE}

\section{DES ESPÈCES ET SYNONYMIES.}

$\Lambda$.

Abyssinica. Brown.

Acicularis. Lindu.

Aciphylla. Rao.

Adenophrlla. IVILLD.

Affinis. Kav.

Agrestis. GMEL.

Alba. Lin.

Alpina. id.

Alpina PaLL.

Alpina lavis. RED.

Alpina pendulina. id.

Alpina $\beta$. Аї.

Altaica. IViled.

Ambigua. LINDL.

Ancistrum. ial.

Andegavensis. Bsst.

Apiifolia. WILD.

Aiborea. Pers.

Aristata. LaPEYR.

Arvensis. Huds.

Arvensis. Schranck.

Arvensis. Linn.

Arvina. Krock.

Atrovirens. VIVIan.

Austriaca. Cranzz.

B.

Balearica. Desf.

Balsamica. IViled.

Buntisice. Brown.

Banksiana.

Belgica. Pronv. .

Belgica. Poir.

Belgica. Brat.

Dengalensis. Pers.

Berberifolla. Pall.

Biflora. Krock.

Eilera. Poir.

Biserrata. Herat.

Blancla. Aï.

Blanda. Soland.

Blanda. Brat.

Blanda. Pursh.

Borreri. IVoods .

\begin{tabular}{r|lr} 
& Pages. \\
Pages. & Bracteata. Wend. & 30 \\
117 & Bracteata. Mornsh. & id. \\
54 & Bractescens. Woods. & 99 \\
99 & Brevispina. Pronv. & 84 \\
136 & Brevistyla. D. C. & 113 \\
99 & Branonii. Lindz. & 120 \\
130 & Burgundiaca. Pers. & 69 \\
83 & Burgundiaca. Roess. & 74 \\
48 & &
\end{tabular}

49 Cæsia. Sмгтн.

C.

42 Calendarum. Moensch.

59 Campanulata. Ена.

140 Candida. Scop.

140 Candolleana. Thоrт.

98 Canina. Lisw.

1 fo Canina. Tиомв.

138 Canina $\beta$. Suter.

45 Canina $\beta$. D. C.

$I 14$ Capreolata. Neill. .

98 Carolina. Linde.

43 Carolina. Durol.

73 Carolina $\gamma$ et $\delta$. Aiт.

I 8 Carolina \&. Аїт.

$7^{3}$ Carolina ל. ill. Lawr.

Caroliniana. Mıch.

Caroliniana. Big.

Caryophyllea. Poir.

118

Caucasea. Lindu.

- 140 Caucasica. PalL.

128 Celsii. Lem.

129

68

6

Centifolia. Linda.

Centifolia. IINN.

Centifolia minor. Roess.

Centifolia $\tau$. Redout.

Cerea. Roess.

Chamærhodon. IViLz.

Chamnagana. Pronv.

Cherokensis. Doon.

Chinensis. Provv.

Chinensis. JacQ.

Chlorophylla. Enr.

Cinnamomea. Linn.

Cinnamomea. Rотн .

Cinnamomea. LourelR.
99
67
-6

51,140

114

98

128

Iof

$9^{\circ}$

118

39

37

37

52

37

39

69

95

id.

140

$7 \mathrm{I}$

69

69

69

87

59

110

125

106

III.

87

43

87

I35 
Cinnamomea. Hermann.

Cinnamomea $\gamma$. Rev.

Clinophylla. Red.

Collina. Jace.

Collina. Sмитн.

Collina. Schr.

Collincola. Err.

Corallina. WILld.

Corymbifera. GMEL.

Cory mbosa. Bosc.

Corymbosa. EHR.

Crantzii. Schultes.

Cuprea. Jace.

Cuspidata. Ровв.

Cymbifolia. Lem.

b.

Damascena. Mirx.,

Daurica. PALl.

Dibracteata. D. C.

Diffusa. Roxв.

Diversifolia. Vent.

Divionensis. Roess.

Doniana. Woops.

Dubia. Wabel.

Dumalis. Becist.

Dumetorum. ThUill.

Dumetorum. Sмгтн:

Dumensis. Dod.

E.

Eglanteria rubra. Roess.

Eglanteria. Micl.

Eglanteria. Linv.

Eglanteria punica. $R$ ED.

Enneophylla. RaF' N.

Eriocarpa. Linde.

Evratina. Bosc. Pronv.

F.

Fecundissima. Moenscr.

Fotida. llerm.

Foticla. Bat.

Fasligiata. id.

Farinosa. Rau.

Fenestrata. Doon.

fierox. Lawrence.

Flava. Doon.

Flexuosia. Rau.
Page.

59

104

134

99

II3

59

140 Fraxinifolia. Dum. C.

99 Fraxinea. Willd.

42 Fusca. MoEnsGh.

39

89

$2^{3}$

$9^{5}$

140

Gallica. Lins.

Gallica hybrida. SER.

Gemella. WiLLd.

Geminata. Rav.

Glandulifera. Rохв.

G.

67

45

113

119

108

69

65

59

$9^{8}$

99

90

59

Glandulosa. Bf LL.

Glandulosa.DC.

Glauca. Dese.

Glauca. Lo1sel.

Glauca. Lo1SEL. Woucens. Wr. Wor, 998

Glaucophylla. Vincr.

Glaucophylla. Eнr.

Clutizosa. Smitu.

Gracilis. Woons.

Grandiflora. LINDL.

Grandiflora. WALr.

Grevillii. HeNT.

H.

S9

Hai-Tong-Hong. Sinens.

$40,13 i$

114

121

49

137

104

99

55

$9^{5}$

77

62

89

120

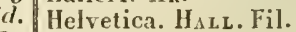

87 Hcmisphrerica. Her M.

87 Herporhodon. EHR.

40 Heterophylla. Woons.

1 qo Hibernica. Sмпти.

Hibernica. Іоок.

Hispanica. MiLL.

Hispida. Pour.

llispida. Moensch.

43 Hispida. Cunt.

87 Hispida. Krock.

79 Holoserica. lioess.

99 Hudsoniana. Thor. Renoute. 33 ,

138 Ilumilís. Marsh.

123 Hybrida. Vize.

25 Hybrida. Schleicn.

-119 Hystrix.

8y llystrix. Linul.
104

114

90

95

114

79

85

Go

13 I

133

53

40

73

39

37

48

114

127

1 ? 
I.

Indica. Liñ.

Indica. Burm.

Indica pumila. RED.

Indica. Forsk.

Indica. Provv.

Inermis. Rox B.

Inermis. MiLL.

Inndora. $\Lambda_{G A R T H}$.

Involucrata. Thory.

Involucrata. Roxi.

Involuta. Sмıтн.

Involuta. Vinch.

h.

Kamtschatica. VENT.

Kamtscliatica. Doos.

Kamtschatica. Red.

L.

Levigata. Мıсн. Lagenaria. IVizL.

Laurenceana. Swelt.

Laxa. Liviv.

Leucanthi.. Loss.

Leucocliroa. Desv.

Longifolia. W Wúd.

Lucidlu. ЕнR.

Lucida. LAwr.

Lucida. $\Lambda$ NDR.

Lutea. Mizu.

Lutea. Brat.

Lutea bicolor. JACQ.

Luteo-nigra. Pronv.

Lutescens. Pursh.

Lutetiana. Lem.

Lyellii. LINDL.

Lyonii. Pursi.

M.

Macartnea. Dum. C.

Macrocarpa. Meur. Cat.

Macrocarpa. Mer.

Macropliylla. LiNDz.

Majalis. heтz.

Majalis. Herm.

Malmundariensis. THов

Marginata. IVALR。

Nicrantha. Smitr.
145

Pages.

1,8

104

128

Millesia. Linn. 140

Mollis. Smith. 79

Mollissima. Bork. 79

Monspcliaca. Gouan. 48

I) 4

Montana. DC. • 89

3о Montezumce. Hомв. et b. 97

29 Moschata. Mill. 121

63 Multiflora. Thомв.

65 Multiflora. Reyn. I 40

Muscosa. Milz. $\quad 69$

Mutabilis. Meur. Cat. I 40

Mutica. Fl. dan. $\quad 46$

27

Mrriacantha. DC. Pronv. $\quad 62$

27 Myrtifolia. IJaLl. 90

25

N.

Nankinensis. LouR. $\quad 6_{2}$

124 Neglecta. Lind. $\quad 140$

48 Nemoralis, Ill. Id.

I10 Nemorosa. Lejeune. 89

36 Nitens. Merat. 99

99 Nitida. WViLLD. 32

id. Nivalis. Doon. 63

rog Niven. DC. 125

35 Noisettiana. Bosc. 107

3o Nuda. Woods. . 99

I of

87

87 Obtusifolia. DEsv.

38 Odoratissima. SweEr. 106

57 Odoratissima. Scal. 89

140 Olympica. Doon. $7^{3}$

3 I Opsostemma. EнR. 12,

13 I Orbessanea. Redout. т 39

P.

3o Palustris. Moenscir. 39

140 Palustuis. Висн.

9o Parviflora. Eur. 37

47 Parvifolia. Id. Lindu. 37,74

46 Parvifolia. Linde.

43 Parvifolia. Palz.

14 Pendula. Rотн. 51

65 Pendulina. Liñs. 19

89 Pensylvanica. Mín. 3y 
Pimpinellifolia. Linn. Pimpinellifolia. PALL. Pimpinellifolia. VILI. Pimpinellifolia. Boв. Pimpinellifolia $\delta$. Red. Platyphylla. Rau.

Pollinaria. Spreng.

Polyanthos. Rioess.

Polyphylla. Wized.

Pomiferá. Herm.

Pomponia. D.C.

Poterium. Lind.

Prostrata Lindi.

Provincialıs. Mill. Pronv.

Provincialis, Lamarck.

Provincialis B. Sмитн.

Provincialis $\gamma$. id .

Pseud'Indica. Linde.

Pseudo-rubiginosa. Lez.

Psilopliylla. Kau.

Pubescens Leman.

Pulchella. Wilud.

Pulchella. IVoods.

Pulverulenta. Buв.

Pumila. Linn.

Punicea. Milz.

Pusilla. M I u r. Cat.

Pygmaa. Buв.

Pyrenaïca. Gouan.

Pyrenaïa 6. Smiti.

I.

Ramanas. Japon. TEUмв.

Rapa. Bosc.

Recurva. Kox.

Reduteana. Thor.

Redutea rubescens, id.

Remensis. Desf.

Repens. GMel.

Repens. Moensch.

Reversa. IV , et K.

Reynieri. HAL.

Rosenbergiana. Thor.

lioxburgliii. Hont.

Rubella. Sмгтн.

ñubicunda. $1 I$.

Rubifolia. Brown.

Rubiginosa. Lins.

Rubiginosa cretica. RED.

Rubra. LaM.

Rubra lucida. Roess.

\section{4}

\begin{tabular}{r|rr} 
Pages. & & Prges. \\
59 & Rubrifolia. Vil. & 103 \\
id. & Rubrispina. Dosc. Nors. & 32,84 \\
49 & Rugosa. Tiumb & 20 \\
62 & Rupestris. Crantz. & 48 \\
59 & Rustica. Leman. & 140
\end{tabular}

S.

132

69

51 Sabini. Woods.

65

77 Sanguisorbxfolia. Doos. 6c

69 Sarmatarea. IVoods. $\quad 99$

140 Sativa. Dodon.

11 Scabriuscula. Sмгтп. 79

51 Scandens. Moensch: 114

69 Scandens. MiL. $\quad$ II8

id. Scotica. id. $\quad 59$

id. Semperflorens. Pronv. 108

130 semperflorens carnea. Roess. Io6

82 Sempertlorens minima. $i d$. $\quad 110$

99 Sempervirerts. LinN. II8

I40 Sempervirens. hau. I38

137 Sempervirens. Rioess. $\quad 114$

79 Sempervirens. Котн. 89

9 Senticosa. Асн. 99

$7^{3}$ Sepium Tnull. $\quad$ 9o

87 Sepium. Tionk. $\quad 99$

110 Sericea. LINDL. 105

49 Serpens. EHR. $\quad 114$

- ill. Seligera. Mich. 126

49 Shirazensis. Kоем PF。 66

Simplicifolia. SALISB. $\quad 23$

Sinica. AIt. 125

Sinica. Livn. 107

26 Solstitialis. Bess. 99

33 Spinosissima. LinN.

$1_{2} 6$ Spinosissima. Lotr. $\quad 136$

6o, 134 Spinosissima. Gort. $\quad 46$

32 Spinosissima. Noensch. 63

74 Sjinulifolia. THOR. $\quad 8_{2}$

I 4 Stipularis. Merat. 90

3 Siricta. Munz. 52

64 Sitylosa. Drsv. 1 i 3

89 Siylosa B. id. $\quad 99$

I 40 Suaveolens. Porsh. $\quad 89$

120 Suavifolia. Ligntfoot. $\quad 89$

5 I Suavis. WILLd. 52

104 Sulphurea. A IтоN. $\quad 55$

123 Subvillosa. Lind. $\quad 140$

89 Surculosa. Woods. $\quad 99$

95 Sylvatica. GAT.

73 SyIvestris. HERM. II

35 Systyla. Lind. II3 
$\boldsymbol{x}$.

Taurica. Bu в.

Teneriffensis. Doon.

Tenuiglandulosa. MER.

Ternata. Polr.

Tomentella. LÉ MaN.

Tomentosa. Smiti.

Trachyphylla. Rau.

Trifoliata. Bosc.

Triphylla. Roxв.

Tuguriorum. IVILLD.

Turbinata. Als.

Turbinata. WiLx.

Turgida. PErs.

V.

Varians. Pонц.

Verticillacantha. Méat.

Velutina. Crainy.

Villosa. Liña.

\section{5}

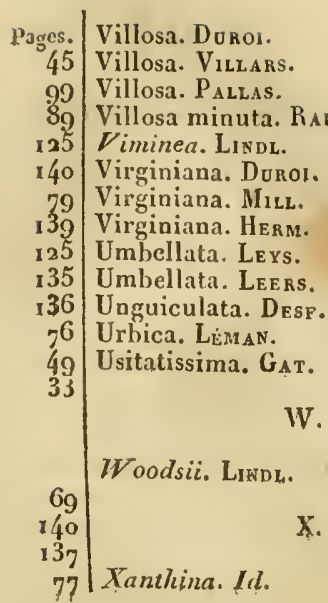

Poges.

79

$9^{\circ}$

79

39

42

52

99

89

69

140

83

38

$13 a$ 


\section{APPENDICE}

SUR LES ESPÈCES, VARIÉTÉS ET SOUS-VARIĖTÉS

CULTIVÉS DANS LES ENVIRONS DE PARIS. - 1824 .

M. Lindley donne une nomenclature des roses doubles cultirées aux environs de Londres, et il l'a placée à la fin de sa monographie; ce n'est, dit-il, qu'à la sollicitation et d'après les instances de ses amis qu'il a publié ce catalogue, car il savait que 11 . Sabine prépare depuis loug-temps un travail sur ce sujet, trarail qui devra intéresser tous les amateurs, vu l'innombrable quantité de variétés qu'il cultive à North-Mims dans le comté d'Hereiord. J'ai cru devoir imiter MI. Lindley arec d'autant plus de raison que nous sommes très-riches en variétés cultirées; l’appendice que je présente ici est également le liuit de: Jongues observations, et quoiqu'il soit plus de la compélence du fleuriste que du botaniste, il ne me paraît point déplacé à la suite de cet ourrage, et il peut être considéré comme le précurseur du grand travail que l'on attend de M. Sabine.

Mais je dois entrer auparavant dans quelques détails sur les principales collections de Rosiers.

Je crois que l'on ne s'est occupé de la multiplication des espieces et des variétés du rosier, que depuis cinquante ans environ; du moins nous ne royons pas de catalogues de roses donbles, arant celui que Miller a publié sur les plantes cultivées au jardin royal de Clielsea; et, parmi les collections des amateurs, on remaryuait alors $(1-64$ ) relle de $M$. Telson prés de Londres. Les pépinieristes de Harlem en Hollande avaient obtcnu des scmis du gallica et de quelques autres espéces, un très-gratid nombre de variélés, comme on peut le remarquer dans le catalogue des frires Van-Eden. On peut présumer que la Hollande et l'Angleterre ont fourni les premières collections de rosiers a fleurs doubles, avec ces noms bizarres et quelquefois ridicules dont les jardiniers bollandais les ont baptisés.

Cependant MM. Richard père et fils établissaient à Trianon une belle collection de rosiers, tandis que M. Dupont 
les faisait connaître à Paris. M. Dumont de Courset n'avait pas oublié de rassembler les espèces et variétés de ce charmant arbuste, dans son jardin botanique situé près de Boulogne. L'on voyait s'accroître de jour en jour cette belle réunion de rosiers, qui se firit admirer au jardin du Luxembourg. angmentée par les semis de M. Charpentier, et suivie avec plus de zèle encore par M. Hardy, chef de ce bel établissement. Depuis environ vingt-cinq ans, le goat pour les rosiers s'est porté à un tel point, qu'indépendamment de la capitale et de ses entours il n'est pas une province, un canton même en France, où l'on ne puisse trouver une collection digne d'arrêter les regards de l'amateur. Il serait impossible de donner des indications sur toutes; je me contenterai de citer les principaux élablissemens de rosiers, les jardins royaux et particuliers, et les pépinières où le public pent s'en procurer.

$1^{\circ}$. Le Jardin royal des Plantes de Paris : Mil. Thouin, directeurs. On y remarque quelques especces étrangères d'une grande dimension, telles que le $\boldsymbol{R}$. bracterta.

$2^{\circ}$. Le Jardin royal du Luxembourg: M. Hardy jardinier en chef. Il renferme la collection la plus complète que je connaisse, soit en espèces botaniques, soit en variétés cultivées.

$5^{\circ}$. Le Jardiu fleuriste du hoi à Sivres, parc de Saint-Cloud: M. Lécolfé jardinier en chef. La collecion a prospéré longtemps sous la direction de II. Lelieur de Ville-sur-Arce; elle renterme plusicurs variélés de rosiers encore peu connues.

$4^{\circ}$. Succursale du Jardin fleuriste, porte Jaune, parc de SaintCloud : M. Putaux jardinier en chel. L'immense collection de rosiers que l'on y remarque, est, je crois, un duplicata des autres pépinières; inais j’y ai remarqué des varićtés assez rares.

5\%. La pépinière de Trianon : jardinier en chef M. Gondouin. Lorsque l'école des arbres et arbustes de cette pépinière sera définitivement établie, je ne doute pas qu'elle ne devienne une des plus intéressantes pour l'amateur comme pour le botaniste : l'espérance que j'en conçois suspend les regrels que m'a causée la destruc!ion de l'ancienne école, si bien dirigée par M. Bosc.

Parmi les collections particulieres, on remarque. celle du château de Malmaison, que Dupontavait formée, et qui a été l'une des plus considérables; elle s'était accrue des espèces et variétés que II. Kennedy avait apportées d'Angleterre.

II. le duc d'Orléans, amateur de l'horticulture, a fait planter une belle suite de rosiers dans ses jardins de Neuilly-sur- 
Scine. Hi. Jacques en est le jardinier en chef. L'on roit également de nombrenses collections chez MM. Thory à Clamart, liedouté à Fleury, Lelieur, Lixon et Deschiens ì Versailles, Dubourg à Vaucresson. Il serait facile d'en citer beaucoup d'autres: passons aux pépinières.

Je ne crois pas que sous le rapport de la botanique comme sous celui de l'agrément, il y ait une collection comparable à cclle de $\mathrm{M}$. Noisette; elle réunit les espéces nouvellement récouvertes aux anciennes, et quoique M. Noisette n'ait pas encore pubiié un catalogue de ses richesses végétales, on peut trouver chez lui dans le genre rosier comme dans d'autres, tout ce que l'art du jardinage offre de plus séduisant pour les amateurs.

il. Boursault possède dans sa magnifique collection de la rue Blanche des rosiers fort rares et qu'il a cultivés avant les grands pépinéristes.

Celle de H. Godefroy de Villedarray, formée avec soin ct rangée avec méthode, est aussi l'une des plus complètes que je connaisse; mais la plus considérable, sous le rapport des varictís de semis, cot sans contredit celle de M. Vibert à Chènevières-sur-Marne. Le l'ond de cette immense réunion de rosiers est l'ancienne collection de Descemet à Saint-Denis, qui passait alors, et à juste titre, pour la plus reinarquable des environs de Paris. Les variétés que H. Vibert à obtenues de scmences sont en bien plus grand nombre; et chaque année, à l'époque de la floraison, tous les jardiniers et amateurs ne manquent point de se rendre à Chènevières, où ils n'éprouvent d'autre embarras que celui du choix.

Les environs de Paris ne sont pas les seuls où se trouvent des pépinières de rosicrs; ou parle beaucoup de celles de Rouen, et parmi celles-ci de la collection de M. Calvert. Dans les départemens dı Haut et du Bas-Rhin, les frères Baumann en cultivent un grand nombre (1), et l'on n'a pas cncore tout observé en fait de roses, si l'on n'a visité les jardins de Gand, de Bruxelles et d'Enghien où II. Parmenticr a rassemblé une si grande collection de plantes direrses, que leur réunion peut se comparer à celle de M. de Courset près de Boulogne.

Mais les grands pépinéristes qui veulent procurer aux ama-

(1) Nous arons un catalogue des roses cultivées à Dôle en FranclieCointé par M. Lerouge, propriétaire et organiste de cette ville. MN. Nicllez, jardiniers élablis à Esquermes près de Lille, possèdent une des plus belles collections que j'aie vues. 
teurs français des plantes rares, et qui se rendent en Angleterre pour y chercher les productions du Calp et de la Nouvelle-Hollande, dans les vastes collections de MM. Kentredy et Lée, et surtout dans le nouvel établissement de M. Loddiges, y trouveront aussi des rosiers de l'Inde et de la Chine qui n'vnt jamais paru en France, comme ils y ont trouvé des variétés singulières, telles que le pompon mousseux, dont te prix est eucore assez élevé pour ne pas être universelleınent répandu. Le eatalogue des rosiers de cette pépinière se monte, dit-on, it louze cents variétés. Pour peu que les jardinier's continuent leurs semis, je ne vois point de terme i cette nomenclature; et, après avoir réussi pendant quelque temps, et par une observation minutieuse, i laire voin les rapports inlimes qui existent entre la plupirrt de ces variétéș, ce genre de travail deriendra tout-it-fait impossible. Je me borne donc a celui que je soumets ici aux amis du genre rosier. Pour un botaniste, l'on conviendra yue j'ai fait une assez grande part aux roses a fleurs doubles, et l'un me saura peut-être quelque gré d'une recherche longuc, souvent monotone, et qui m'a mis parfois sur les épines. 


\title{
ABRÉVIATIONS DE L'APPENDICE.
}

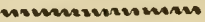 \\ (Hort.) De mon jardin à Versailles. \\ (H. R. école.) École du jardin royal à Paris . \\ (Pot. R.) Potager du roi à Versailles. \\ (Lux.) Jardin fleuriste du Luxembourg. \\ (Sèvres.) Jardin fleuriste du roi à Sèvres. \\ (Pép. Trian.) Pépinière du jardin de Trianon. \\ (St.-Cloud.) Pépinière de St.-Cloud, porte jaune. \\ (Pép. Nois.) Pépinière de M. Noisette à Paris. \\ (Pép. God.) Pépinière de M. Godefroy à Villedavray . \\ (Pép. Vib.) Pépinière de II. Vibert à Chènevières. \\ (H. Bours.) Jardin de M. Boursault à Paris. \\ (H. Lix.) Jardin de M. Lixon à Versailles. \\ (H, Thor.) Jardin de M. Thory à Clamart. \\ (H. Malm.) Jardin de la Malmaison.
}




\section{SOM M A I R E}

DES ESPECES, VARIÉTES ET SOUS-VARIÉTÉS

DU GENRE ROSIER. - JUILIET 1823.

\section{PLANTES INDIGENES ET CULTIVEES.}

PREMIERE SECTION.-FRUITS GLOBULEUX OU OBRONDS.

$1^{\mathrm{re}}$. 'Tribu. - Rosiers à feuilles simples. Feuilles simples, sans stipules. Réceptacle nu.

เ. IRosa berberifolia. - ROSIER A FLEURS SIMPLES. Perse. H. R. Id. Malmaison.

Cc rosier, dont l'espèce est unique, est fort rare dans les collections à oause de la difficulté de le multiplier.

$$
2^{\circ} \text {. TRIBU. - Rosiers féroces. }
$$

Rameaux couverts d'un duvet persistant. Fruits nus. II. I R. ferox. Lindl: - R. FÉROCF. -

R. du Kamtschatka des jardins.

2 Id. Variété double. Luxembourg.

Il est plus commun que le véritable kamtschatica.

4 III. I R. rugosa. Thumb. Jap. - R. A FliULLES liIDEES. Somm. p. 9. Herb. Banks.

Il n'a pas encore été cultivé en Angfeterre ni en France.

5 Iv. I R.kamtschatica.-R.DUKAMTSCHATKA. Somm. p. 9. Hort. bine.

Celui-ci m'a été envoyé d'Angleterrc par M.Sa- 
3. TriBu. - Rosiers bractéolés.

Fruit et rameaux couverts d'un duvet persistant. Bractées presque verticillées et pectinées.

v. I R. involucrata. Somm. p. 10. Népaul. Jard, de Sabine.

Ce n'est pas le R. involucrata de M. Thory.

VI. I R. bracteata. W. - R. BRACTÉOLÉ. R. Macartnea. Dum. Courset. Chine.

Ce rosier est du Boutan, et sa variété $\beta$ de la

2 Id. Var. scabriusculis. Lindl. H. R. - Id. H. Noisette. tions.

Celle-ci est plus connue dans les grandes collec-

9 vil. I R. Lyellii. Lindl.-R. DE LYELL. Népaul. Herb. de Bauks.

Ce rosier n'a pas encore été cultivé en $\Lambda$ ngleterre.

$$
4^{e} \text {. TribU. - Les cannelles. . }
$$

A écorce rougeâtre, couverts de soies ou à tige nue, munis de bractées ; folioles lancéolées, privées de glandes, de 5 à 7 . Disque très-petit quoiqu'épais. Aiguillons axillaires géminés ou opposés. Obs. Cette tribu est presque toute américaine.

vII. I Rosa nitida. - R. BRILLANTE. A Terre

Neuve. Somm. p. 12.

Cultivée dans le jardin de M. Sabine.

1 1x. I R. rapa. - R. TURNEPS. Bosc. Somm. p. 12. Provinces méridionales des ÉtatsUnis.

Nous n'avons pas d'individus à fleurs simples.

2 Variété A FL. PLUS Grandes. Luxembourgę. Jard. fleuriste. 
x. I R. lucida. - R. LUISANTE. Caroline; près Boston. Somm. p. I2.

Fleurs șimples, les dernières en corymbes nombreux.

2 R. hudsoniana. - R. d'Hudson. Baie d'Hudson. Thory. Prodr.

Pép. Vibert; fleurs doubles, peu éclatantes; corymbes nombreux et terminaux.

R. Iaxa. - R. ÉTALÉ. Somm. p. x 3. Amérique sept.

Chez .1. Noisette; il me vient de M. Sabine.

xu. I K. parviflora. - R. A PETITES FLEURS.

A Neubourg; en Caroline. Somm. p. 13.

Fleurs doubles. R. pensylvanica des pépinières; est délicat; il a existé à fleurs simples dans l'ancienne école de Trianon?

17 xill. I R. Woodsii. - de Woods Lindl. Mon. Somm. p. 13.

Près du Missouri. Am. sept.

f. luten nigra de Noisctte; folioles arrondies ct fortement dentées; fleurs simples.

xiv. I R. Carolina. - R. DE CAROLINE. Somm. p. 14. V. palustris.

C'est le R. corymbosa des pépinières.

2 - Grandes fleurs. R. virginica de Godefioy.

Perdue à Trianon. Il est bifère ; buisson épais.

4 R. gemella. - R. Garcuive à 2 leurs. Pép. Noisette. Am. sept.

losier nain ; fleurit en juillet. Baie d'lludson.

liosicr élevé; se trouve en $\Lambda$ ngleterre, jardin de Sabine. V. S. dans l'Herlier général. II. R. 
23 Xvı. I R. fraxinifolia. - R. A FEUILLES DE FRÊNE.Somm.p. 15. - A Terre-Neuve. Lindl.

Élevé; tige rouge : c'est le cinnamomea des pépinières.

24 xwI. I R. cinnamomea. - R. CANNELLE. Somm. p. 15. en Suede, Auvergne.

N'est cultivé dans aucun jardin?

2 - Var. fluvialis. Danemarck.

Feuilles aiguës; Fl: dan.

26 xvilr. I R. majalis. - R. DE MAY. - DU SAINTSacrement. Somm. p. I6. Suède.

Notre R. du St.-Sacrement, qui est double, aptient-elle au cinnamomea?

2 - Var. B. canescens. Ibid. p. 16. En Lapo. nie. Hort. Sabine.

Cette variété $\beta$ a fleuri chez moi cette année.

3 - A fleurs panachéEs. H. Noisette. Variété de semis.

29 xIx. I R. macrocarpa. - R. A GROS FRUITS. Somm. p. 16. Gossan-Than. Indes or. Wallich cité par Lindley.

Herb. de Banks; a été confondı avec le majalis sous le nom de R. du Nissouri; Hort., Noisette.

$$
5^{\mathrm{e}} . \mathrm{T}_{\mathrm{RIBU}} \text { - Les pimprenelles. }
$$

Couverts de soies. - Tiges nues ou munies d'aiguillons très-rapprochés et presque semblables. Point de bractées ou très-rarement. Folioles ovales ou oblongues de 5 à 13 . Sépales conniventes et persistantes. Disque presque nul.

3o xx. I K. alpina. - R. DES ALPES. L. Somm. p. 17. France mérid.

Je pense que le pendulina est le mème que le type.

2 - Var. B. pyrenaïca. R. Des Pyrentés. Sornm. p. 18 . Lapeyr. Hist. P. Cullivé à Attichy, chez M. Pelletier. 


\section{5}

3 - Var. C. lagenaria. - R. en forme de bouteille. Alpes du Piémont.

Je l'ai reçu des environs d'Aost. Villars Delph.

4 - Var. D. pimpinellifolia. Idenı.

Plus petit; il a les fruits ronds, et me vient de M. Vibert.

34

5 - Var. E. sinensis de Dupont.

Fleurs doubles; très-élevé.

35

6 - Var. F. rossica. - R. DEs Alpes de Russie. R. de Pallas. Pép. Noisette.

Il porte quelques aiguillons épars.

xxI. I R. rubella. - R. A FLEURS ROUGES. Somm. p. 1S. Angleterre; Sibérie.

37

2 - Var. $\beta$. melanocarpa. Idem.

Cultivé ehez M. Noisette; c'est le R. candolleana. La var. D chez M. Sabine.

xxil. I R. stricla. - R. A RAMEAUX GRELES.

Sornm. p. 19. Pensylvanie; Am. sept.

Fleurs petites, serrées contre les rameaus; il a fleuri cette annce $18_{2} 3$.

39 xxi!r. $\times$ R. acicularis. - R. ACICULAIRE. Somm. p. 19. De Sibérie.

Aiguillons en forme d'épingles; fleurit à la fin d'avril ; je l'ai communiqué à plusieurs établissemens.

40 xxiv. I R. sulfurea. - R. JAUNE DE SOUFRE. Somm. p. 19. Orig. d'Orient.

41

2 - Var. B. minor. Pourpre jaune.

La var. B ne fleurit que greffée, et rarement; elle est double comme la première.

42 xxv. I R. lutescens. - R. JAUNATRE. Lindl. Somm. p. 20. Am. sept. En Sibérie.

Fleurs simples, jaune très-pále; je ne l'ai pas encore multipliée.

43 xxvr. I R. viminea. - R. SARMENTEUX. Somm. p. 20. Lindl. Mon. Patrie inconnue.

Peut-être le rosier du Fleuriste de Sévres est-il celui-ci ? 


\section{6}

44 XxvIr. I R. spinosissina. R. TRES-ÉPINEUX. -

R. Pimprenelle. Montagnes et forêts d'Europe et d'Asie. Somm. p. 20. Var. I. Espèce très-nombreuse en variétés naturelles et cultivées.

$\mathbb{S}$ I. Pédoncules soyeux ou épineux.

45

46

2 - Pimprenelle a fleurs blanches. Somm. p. 2r. A Fontainebleau.

Commun dans les montagnes de l'Écosse.

3 - Petite écossaise blascie double. Assez commune dans les pépinières. 4 - Carnea bifera. Estelle de Vibert.

$\$$ 2. Var. Pédoncules nus.

5 - Var. carnea. Pimpipenelle carnée. Fleurs simples.

Touffle basse ; fleurs simples.

6 - Idem, fleurs semi-doubles. Fleurs plus grandes; tige élcvée.

7 - Austriaca. Pimprenelle d'Autriche. R. pimpinellifolia. H. R.

Très-grand; rejetons couverts de soies; les rameaux nus; fleurs blanches striées de rougc.

8 - Var. a rleuns elanches maculćes de rouge. Belle Laure de Dupont.

Assez grand ; aiguilloṇs mêlés de soies.

52

9 - Var. reversa. Somm. p. 21. R. reversa. Noisette. idem. Lux.

Fleurs simples, blanclies; buisson épais, trèsépineux.

xo - Var. platycarpa. Luxembourg.

R. Hispida. Poiret. Thory. Prodr.

Feuilles luisantes; pédoncules et tubes hérissés; il appartient à la var. 1.

54 II - Var. pilosa. Somm.p. 21. 
J'ai reçu cette variété de M. Sabine; n'a pas fleuri cette année.

56

57

58

59

60

61

62

63

64

65

i6

o

6 i

2

$$
13 \text { - Var. rossica. Idem. }
$$

14 - Var. islandica. Idem. N'est pas le R. hybernica de Lindley.

15 - Var. sanguisorbafolia. Idem.

Je n'ai pu vérifier si c'est le sanguisorbafolia de H. Noisettc.

16 - Var. redutea. - R. red. glauca. Thory. Prodr. p. 43. Toutes ces variétés sont décrites dans la Monographie de Lindley. Il a l'aspect d'un rubrifolia, mais il ne me parait pas devoir constituer une espèce.

17 - Pimpinellifolia. Var. a fleurs pourpres foncé. Vibert.

Feuillage d'un vert foncé; fleurs simples.

18 - Var. a fleurs jaunes doubles.

Jardin Sabine en $\Lambda$ ngleterre. Le rosier de Vibert n'appartient pas à cette espèce.
19 - Pimprenelle camella. Jardin du Luxembourg.
Fleurs doubles, blanches, très-régulières.
$320-$ Micrantha. - R. A PETITES FIEURS POUR- PRES nuancées.
Très-jolie variété, qui a fleuri clıcz moi.

421 - A petites fl. Blancies, panachées de rouge. LEs 40 Écus. Luxembourg.

22 - Pimprenelue Nankin. Lux.

Fleurs carnćes, sensiblement jaunâtres, simples. 
68 xxix. I R. myriacantha. - R. A MILLE ÉPINES. Somm. p. 12. Sibérie. Pall. et Dauphiné. Decandolle.

Herbier de Hooker et Lambert; n'est pas celui de M. Desvaux.

69 xx. I R. involuta. Sibthorp. Écosse. Somm. p. 23. - R. A FLEURS EN CUUPE.

Très-épineux; pédoncules et fruits armés de soies; fleurs blanches, simples.

7o xxxi. I R.Sabini.-R. DE SABINE. Somm. p. 23. Nord de l'Angleterre. L.

La var. 2 a fleuri cette année.

2. - Var. doniana. Idem. Wood.

Cette espèce a des rapports avec la précédente.

DEUXIEME SECTION. - FRUITS OVALES ou VARIESS DE FORME.

6:. Tribu. - Les centfeuilles.

Munis de soies. Aiguillons de plusieurs formes. Pédoncules bractéolés. Folioles 3-5-7 ovales ou oblongues, ridées. Disque épais couvrant l'entrée ou l'orifice du réceptacle. Sépales multifides.

$7^{2}$ xxxil. I R. damascena. - R. DE DAMAS. Somm. p. 24. La Syrie. Lindl. - R. bifera. Pers. syn. - R. 4 salsons. Fl. rouges.

Très-commun dans les jardins, mais confondu mal à propos avec l'espèce suivante.

732 - Var. A FL. Blanches. Hort. Beaucoup plus grand que le précédent.

74 3 - Var maxima. - La gracieuse de Vibert. Hort.

Var. de semis, dificile à multiplier.

y5 4 - Var. inermis. - Le Damas sans Épines. Hort. Vibert. Nouvelle variété. 

Nouvelle variété. Vibert.

6 - Damascena Portlandica. - R. De PontLand. Somm. p. 25. Angleterre. Semidouble.

Fleurs pourpre-ćcarlate, se succédant jusqu'en novembre, ainsi que les suivantes.

7 R. damascena. Var. porllandica rosea. R. Perpétuflle. Godefroy.

Fleurs roses, semi-doubles; fcuilles d'un vert clair.

8 - Portlandica maxima. - R. DU Rot. Fleuriste de Sèvres.

Bellc variété , fleurs pourpres, très-doubles.

So xxxill. I R. belgica. - R. BELGIQUE. - Damas des jardiniers. - R. de Puteaux. Brabant. Somm. p. 24. Cultivée au pied du mont Valérien.

8,

2 - Var. argentea. - Damas argenté. Rose à bouquets des jardins.

Donquets nombreux: Floraison assez longue par la succession.

82. 3 - Var. purpurca. - LE DAMAS POURPRÉ de Vibert. Hort.

Fleurs-éclatantes mais peu nombreuses.

4 - Var. carnea. - Belgrque carné, de Godefroy. Hort.

Plus vigoureux; fleurit beaucoup et long-temps.

5 - Var. violacea.-Belgique riolet.Trianon. Hort.

Blanches, et pourpre - violet intérieurement; très-doubles.

6 - Var. nutabilis. - R. DE CELs. - BeLle Covronnée. Hort. Cels.

Fleurs nombreuses, carnées, blanches ensuite.

86

7 - Var. variegala. - FÉlicité.

Fleurs roses avec des nuances carnées; veut être greffé. 
8 - Var. eicolor. - Yorck et Lancastre. Angleterre. Miller.

Ancienne variété; toujours recherchée.

88

9 - Var. La belle auguste. Vibert.

Variété de semis; très-double, régulière, carnée; a du rapport avec le $\mathrm{N} \circ .4$.

10 - Var. La petite couronnée. Fleuriste de Sèvres.

Fleurs moyennes, plus doubles, plus colorées, mais ayant des rapports avec le No. 6 .

90 xxxiv. I R. centifolia. - R. A CENT FEUILLES, Somm. p. 26. Caucase. Rau. Énum. rose.

C'est celui que les jardiniers appellent unique

2 - Var. hollandica. - R. DE Hollande. Gros centfeuilles. Hort.

La plus belle quoique la plus commune des variétés du rosier.

$y^{2}$

3 - Var. maxima. - R. Souchet. - R. DEs peintres. Trianon, - Cent feuilles écarlate. Godefroy.

Semi-double, très-grande, couleur vive.

$9^{3}$

4 - Var. carnea. - R. Vilmorin.

Double; a une sous-variété d'une nuance plus foncée.

94

5 -Var. extùs rubra. - R. nutabilis. Persoon; - R. UNIQUe BLANCHE. lica.

Inflorescence moins nombreuse qque celle du gal-

6 - Var. glauca. - Centfeulules de Hesse. Godefroy.

$9^{6}$

7 - Var. multiflora. D. Courset. - Petite Hollande hâtive des jard.

Les fleurs, trop nombreuses, n'ouvrent pas toujours hien.

8 - Muscosa. - R. muscosa. Willd. - R. MOUSSEUSE, ROUGE. Somm. 26.

Originaire de Provence; a une sous-varićté carnéc et une autre ì fleurs simples. 
9 - Muscosa alba. - R. mousseuse blavche. Angleterre.

Très-délicate.

10 - Var. foliacea.-R. Folí́. Bosc. Dict. Duchesse de Berri. Tamponet.

Originaire de llollande, et donnée depuis trois ans comme nouveauté.

II - Var. prolifera. - La mère Gigogne. R. MovstrueUse.

Variété plus singulière qu'agréable.

IoI

12 - Var.caryophiyllata. - Rose oeILlet et sa sous-variété. R. apetala. R. SANS PÉtALES, Fruits en amphores, ou cruches antiques; provient de la $R$. des peintres, Nंo. 3 .

13 - V. bullata. - R. A feuilles DE latue. Fleurs doubles, bien faites, mais peu nombreuses.

14 - Var. belgica. - R. centfeuilles de Bruxelles. Hort.

On compte aussi parmi ces variétés : 1 . Centfeuilles de Nancy, God.; 2. Centfeuilles gaufrée, Noisette.

15 - Var. anemonceflora. - Centreuilles ANÉMonf. H. Noiselte.

Semi-double; rose tendre; très-notable variété.

16 - Var. incarnala. - La Constance. - Centfeuilles d'Avranches.

llybride du gallica; l'une des plus grandes du genre.

17 - Var. Cumberland. Angleterre. Hybride; rose clair; bouquets nombreux. A du rapport a vec la précédente. Hybride; la plus grande de l'espèce. 
21 - Var. burdigalensis. - LE GROS POMPON. - R. de Bordeaux.

Aucunc variété; moins commune aujourd'hui.

111

112

113

114

115

I 16

117

118 xxxv. I R.provincialis. -R. DE PROVENCE.Somm.
p. 26. Niller. Dict. H. Noisette.
Fleurs blanches un peu carnées; peu répandu.

118 xxxv. I R.prowincialis. R. DE PROVENCE.Somm.
p. 26. Niller. Dict. H. Noisette.
Fleurs blanches un peu carnées; peu répandu.

118 xxxv. I R.prowincialis. R. DE PROVENCE.Somm.
p. 26. Niller. Dict. H. Noisette.
Fleurs blanches un peu carnées; peu répandu.

119

22 - Var. Kincston. R. de Portugal.

Feuilles de l'espèce; fleurs de pompon.

23 - Var. C. pomponia. Somm. p. 26. - R. pompon . Bourgogne, Durande. Fleurs simples. Hort.

Ce n'est pas le vrai R. burgundiaca, qui est le parvifolia de Lindley.

24 - Var. a fleurs doubles. - R. pompon des jardins.

On l'élève à Paris comme primeur.

25 - Var. lusilanica. - Ponpon de Portugal. - Lisbon rose. Angleterre.

Charmante sous-variété peu répandue.

26 -Var. muscosa. - Ponipon mousseux. Angleterre.

27 -Var. AglaÉAdanson de Vibert.

Grande, scmi-double, d'un rose purpurin, panaché de rose pâle.

28 - Var. R. Charrois. A Versailles. Petite

- plante, traçante; fl. moyennes, doubles, rouges comme la R. de Bordeaux.

$N$. B. Je ne conmais pas le R. centifolia putidula. - R. à odeur de punaise. Thory. Prodr. v. 10.p. 75. R. centif. Junonis. - R. centfeuilles Junon. ND. Idem, p. 7. Et R. centif. perpetua. - R. centfeuilles de Deschallois. Id. p. 96 .

2 - Var. carnea. - R. DE Provence, couleur de chair. H. Noisette.

-Fleurs simples comme le précédent; je les cultive tous deur. 


\section{3}

120

121

122

123

124

125

126

127

I 2.9

128 I - Var. Agatre pyramidale. Saint-Cloud.

3 - Var. inermis. - R. Pomme de grenade. Pép. Godefroy. Hort.

Élevé; fleurs semi-doubles, en corymbes érigés; rose vif.

4 - Var. multiflora. - Agathe royale. Bouquet parfait.

Très-commun ; fleurs nombreuses; rose taché de rouge.

5 -Var. nutabilis. - Duchesse d'Angourène. - Marie-I Louise.

Fleurs très-nomhreuses, blanches et carnées; buisson épais.

6 R. provincialis. Var. Hybride. - Agathe DE Portugal. Hort.

Corymbes nombreux; fleurs très-doubles, roses et carnées; vient du centifolia.

7 R. prolifera. - R. Acatue prolifére. - Précieuse agate. - Agathe favorite.

C'est la même variété connue sous plusieurs noms.

8 R. hybride. - R. agathe de Francfort. Hort.

Fleurs nombreuses et très-doubles; elles ne s'ouvrent pas toujours.

9 - Var. alba. R. de Provence blanche. Fleuriste de Sèvres.

Fleurs trés-doubles; corymbes serrés ; sous-variété un peu carnée; au Luxembourg.

o - Var. spectabilis. - R. De Provence violette remarquable. Saint-Gloud. Porle jaune.

Très-belle variété foliacée et prolifère. Porte jaune.

Fleurs doubles, prolifères, d'un incarnat très-vif.

2.9 - Var. la Parisienne. Au Luxembourg.

Fleurs doubles, noyennes, carnées; sépales et pédoneules allongés. 


\section{4}

130

131

133

134

132 xxxvı. I R. parvifolia. R. A PETITES FEUILLES. - R. de Bourgogne. Somm. p. 27. tion.

A Trianon. Ce rosier est perdu dans la collec-

2 R. flore pleno. - LE petit Saint-Francois. Hort.

Fleurs nombreuses, pourpres; moins connu que le centifolia pompon.

3 - Var. remensis. - R. DE Champagne pomPoN. Des Alpes. Trianon.

Me paraît un hybride du provincialis, mais peutêtre du Tomentosa.

135 xxxvir. I R. gallica. - R. DE PROVINS. - R. GALLIQUE. Bosc. Somm. p. 27.

$$
\text { \I. - Les pourpres. }
$$

136

2 - Var. POLRPRE DOUBLE. - Lústre d'ÉGLise. - D uchesse d'Orléans.

Élevé; fleurs moyennes, peu nombreuses.

137

138

139

3 - Var. Poivt pourpá. Godefroy.

4 - Var. la Junon. Dupont. - CRamoisi triomphant. Polager royal.

5 - Var. LE rot des pourPres. Godefroy. Rexoxcele NoIratre. Lille.

Fleurs moyennes, très-doubles, se succédant long-temps.

140
6 - Var. grand cranioisi. Trianon. - PourPRE SANS ÉPINE. Godefroy. N'est pas la Clémentine de MI. Vibert. 
$14 \pi$

142

I 43

344

145

146

47

148

38

149

150

151

152

7 - Var. pourpre pe Tyr. Potager. Ancienne variété de Hollande.

8 - Var. la copuette. Trianon. - Ancienne ROSE ANEMLNE. Godefroy.

9 - Var. macrocarpa. - Provins a gros fruit. Fleuriste de Sevres.

Fruit oblong, aussi gros que celui du R. pomifera.

10 - Var. gloria mundi, à fleurs rouges. Sa variété à fleurs roses.

A Trianon et ailleurs. Variétés de Dupont.

I) - Var. Bouquet POURPRE.

Obtenue en 1814; a fleuri à Sèvres.

12 - Var. la petite paxachíe. Saint-Cloud. Porte jaune.

Fleurs nombreuses, très-doubles, nuancées et marbrées.

13 - Var. maximus pourpre des Hollandais. Saint-Cloud. Porte jaune. Grandes fleurs, semi-pleines.

14 - Var. punctala. -R. Ponctuée. Belle HerMuvie de Vibert.

Fleurs semi-doubles, pourpre-vif, ponctuées de blanc.

15 - Var. maxima. - Eucharis du Luxembourg.

Grandes fleurs semi-doubles, pourpre-vif, nombreuses.

16 - Var. pourpré charmant, strié de blanc. Fl. du Luxembourg. Variété de semis.

17 - Var. pourpre charmant du Luxembourg. Fleurs doubles; n'est pas le graud Pompadour.

18 - Var. Le RoI de Fraxce. Au Luxembourg. Fleurs très-grandes, pourpres, coulcur égale ; boutons allongés. 


\section{6}

\section{3}

154

155

156

157

158

159

160

161

${ }_{102}$

163

164

165
19 - Var maculata - Provins pulmonaire de Noisette.

Fleurs moyennes, semi-doubles, pourpres, tachées en clair à l'onglet des pétales.

20 - Var. papaverina. - R. PAvot. - GRanDESSE RoYale. Godefroy.

Les plus grandes fleurs de l'espèce.

21 - Var. Manteau pourpre. - Le manteau RoYal. Hort.

22 - Var. ThÉRÈSE. Bar-sur-Aube. H. Lixon. Grandes fleurs pourpre-violet, très-doubles, nombreuses.

\$2. - Les violettes.

23 - Var. violacea. - L'ardoisée. - BüonaParte. - Grand - Alexandre. Godefroy. Fleurs doubles, violet intense, peu nombreuses.

24 - Var. bishops. - R. EVÊQUE. Ancienne variété. Pétales violets, ponctués de blanc.

25 - Var. kayseriun.- R. IMPÉRATRICE.-PhLÉGÉTox. H. Deschiens. Potager du Roi.

26 - Var. nolre de Hollande. Godefroy. Pourpre-violet très-foncé.

27 - Var. la terminale de Vibert.

Semis du variegata; une fleur à l'extrémité de chaque rameau.

28 - Var. L'amable violetre. H. La Haye.

29 - Var. regine dicta. - R. DE LA REINE. Pépinière de Godefroy.

30 - Var. almable de Storr. Vibert.

3 I - Var. enfant de France. Godefroy. Le rol DE RoMe. Trianon.

Fleurs moyenncs, trés-doubles, régulières; pétales ondulés. 


\section{7}

166

167

168

169

170

17 I

172

$17^{3}$

174

$17^{5}$

32 - Var. Joséphine de Saint-Cloud. Semis du fleuriste de Sèvres.

Corymbes de 4 à 5 ; fleurs très-doubles, régulières.

33 - Var. de Pronvilite. Semis du Fleuriste de Sèvres.

Grandes fleurs violet-brillant, nuancées en clair sur les bords; pétales ondulés.

34 - Var. grande ardoisśe striée de blanc. Semis du Luxembourg.

Semis de la R. évêque; a du rapport avec le No. 23 .

35 - Var. Hertwy. Idem. Luxembourg.

Fleurs presque violettes en leurs bords; feuilles d'un vert sombre.

36 - Var. Ninon ue l'Enclos. Vibert.

Fleurs très-grandes, régulières, pourpre-violet nuancé.

37 - Var. Rose de Parade. Idem.

Fleurs grandes, semi-doubles, pourpre-violet.

33 - Var. AglaÉ de Marsilly. Idem.

Fleurs moyennes, lilas nuancé de violet, nombreuses.

39 - Var. la belle Equermorse. Lille. H. Lixon.

Violettes nuancées; ont du rapport avec le No. 33.

\section{\$3. Les veloutées.}

40 - Var. Maheca nova. Godefroy. - L'AIGLE nolr. Noisette.

L'ancien maheca est l'aigle rouge de Dupont; celui-ci est plus foncé en couleur.

41 - Var. pourpre charmant. Grand PompaDour. Godefroy. Hort.

Fleurit beaucoup; variété constante. 
$1-6$

177

178

179

i8o

181

182

183

184

185

186

188

$18753 \mathrm{R}$. inermis. - R. Cuénentive de Vibert.

42 - Var. négrette. Godefroy. - Cramots! Éblouissant. Potager du roi.

Veloutée et foncée. ADv. La négrette du Luxembourg:

43 - Var. le velours pourpre. Godef. - CraMolsi incomparable. Potager.

Semis de Descemet.

44 - Var. LA SUPERbe en bruv. Godef.

Taches brunes sur des pétales d'un crampisi foncé.

45 - Var. LE Graxd sultan. Trianon.

46 - Var. Le velours Noir. Lille.-Le benu veLours. Fleuriste de Sèvres.

Double; velours-puce.

47 - Var. L'obscurité. Trianon.

Semi-double; a du rapport arec le précédent.

48 - Var. cramosissimo amplo de Lille. 'TeMrle d'A pollon. Rouen.

Grandes fleurs doubles, velours éblouissant, très-belles.

49 - Var. carhin brillant. Iille.

Peu connue; fleurs moyennes, très-doutles, nuancées.

50 - Var. Blood. - R. Sanguine d'AngleTERRE. Trianon.

Le R. gallica cruenta du Luxembourg en est une sous-variété.

51 R. holosericea nova. Luxembourg.

52 - Var. mairmorea. Var. de Dupunt.

$\$ 4$. Les roses el carnées. Hort.

Fleurs roses et carnées sur la même tige.

5. - Var. ornement de parade. Godef.

Flcurs très-grandes, un peu plates dans l'épanonissement. 
55 - Var. le grand monarque. Godef. Rose PIvolve de Trianon.

Préférable à la précédente; couleur plus vive.

190

56 - Var. variegata. - Le provins PANACiÉ.

Il n'est encore que semi-double.

19!

57 - Var. argentea. Cent feuilles de ChateNaY. Cat. de Godefroy.

Ancien semis croisé avec le centfeuilles; fleurs carnées, assez nombreuses.

192

58 - Var. porcelaine à bords blancs. H. Godefioy.

Ancicnne variété de Dupont.

$19^{3}$

59 - Var. belle sans flatterte.

Fleurs moyennes, rose-clair, très-doubles, ondulées régulièrement.

60 - Var. amable rovge. - R. hortensia. Godefr. Hort.

Variété assez répandue; fleurs nuancées vers le centre.

6r - Var. striata. - R. mauve. Ancienne pivoine. Hort.

Semi-doublc; fleurs moyennes, roses, striées de pourpre.

63 - Var. la tendresse. Cat. de Godefroy. Ancienne variété de Dupont.

6/4 - Var. Henry iv. Fleuriste de Sevres. Fleurs grandes, semi-doubles, pourpre-clair, tirant sur l'incarnat.

65 - Var. LA DAUPHINE, idem..

Semis de 181 ; f fleurs moyennes, très-doubles, incarnat-clair.

66 - Var. LE nuc de Guiche, idem.

Grandes fleurs très-doubles, rose pourpré.

200

67 - Var. LE bovnuet rose des Hollandais. Sain!-Cloud. Porte jaune: 
68 - Var. R. agréable.-Proviys car.ye, idem.

202

$6 y$ - Var. LA CBÉRIE. Luxembourg.

203

70 - Var. la Pontatowser. A examiner. Fleur du Laxembourg.

7I - Var. R. intalie, idem.

Fleurs grandes, semi-doubles, rose-clair nuancé et marbré.

72 - Var. R. G $\mathrm{G}_{\mathrm{T}}$, idem.

206

73 - Var. R. Favyy Bias. Vibert. Fleurs grandes, très-doubles, incarnates.

74 - Var. R. belle de Descemet, idem.

$§ 5$. Les blanches.

208

75 R. - alba. La fatsse twiQle.

Commune dans les pépinières, mais très-remarquable.

76 - Var. le pojpon bazard. Vibert.

Fleurs petites, trẻ-doubles, blanches un peu rosées.

N. B. Plusieurs autres variétés méritent d'être citées, mais elles ont des rapports si intimes avec celles-ci, que je crains les doubles emplois. J'ai remarqué, toutefois, la PERLE D'ORIENT, le bizarre triomphant. Rose NAPOLÉOY de M. Lixon.

\section{7 . Tribu. Les roses velues.}

Rejetons redressés. Aiguillons presque droits. Folioles ovales ou oblongues. Dentelures divergentes. Sépales conniventes et persistantes. Disque épais, formant l'entrée ou le centre du réceptacle.
210 xxxyu. I R. turbinata. $-\mathrm{R}$. TURBINÉ. P. R. de,Francrort. Somu. p. 29. 


$$
1>1
$$

211

212 Laye.

2 - Var. fl. pleno. - R. dE Frarcfort a FL. DOCBLES.

Commune dans les jardins à Saint-Germain-en-

3 - Var. maxima. - R. GRAsde PIFolve de Lille. Hort. forte.

Fleurs très-grandes, pourpre peu éclatant; tige

213 xxxix, 1 R. villosa. - R. VELU. - R. BISPIDE. R. PoyIfìre. Somm. p. 29.

L'un des plus grands du genre; on fait confire ses fruits.

214

2 - Var. a fl. SEMI-Docbles. Pép. Noisette.

Fruits oblongs, moins gros que dans le précédent.

215 xL. I R. tomentosa. Smith. - R. COTONNEUX. Somm. p. 30 .

Le Tomentosa relutina de Woods en est une rariété.

216

2 - Var. B. mollis. Somm. p. 30.

h. mollissima de Willdenom.

217

3 - Var. microcarpa. - R. 1 PETITS FRUITS. Trianon.

215

4 - Tar. fl.pleno. - R. cotoriecse à fleurs doubles. Lille. Noisette.

M. Noisette possède une sous-rariété à pétales crénelés.

219

5 - Var. C. resinosa. Somm. p. 3o. - R. villosa terebinthia. Thory. Prodr. Var. D.

220

6 - Var. a fl. planches. Noisette.

Jolie variẻté; fleurs moyennes, semi-doubles.

221

7 - Tar. A Gr. Fletrs carvées dotbles. SaintCloud. Porte jaune.

C'est uue sous-rariété du 10. 4 .

222

S - Tar. reversa de Tibert.

Grandes fleurs carnées, très-doubles. 
223 xLI. I R. spinulifera. Thor. Prodr.-R. A FEUILLESEPINEUSES. A Clamart.Ind. en Suisse. Ce rosier n'a encore été publié que dans le Prodrome de M. Thory.

224 xuI. I R. alba.-R. BLANC. Somm. p. 3o. d'Italie. Fr. mér.

Potager du roi et autres jardins.

2 R. alba. Var. belle Hentette. - La cocarde. Potager du roi.

Fleurs simples, boutons roses; corym bes nombreux.

3 - Var. le bouquet blaxc. Fl. Sèvres. SaintCloud. Porte jaune.

Corymbes allongés, 5 à 6 ; fleurs très-grandes, presque simples, d'un blanc éclatant.

4 - Var. albavirginalis. Saint-Cloud. Porte jaune.

louquets nombreux de fleurs semi-doubles.

5 - Var. regia.-La royale. Bot. cult. $n^{\circ}$. 2 . Dans tous les jardins.

Issu du No. I.

6 - Var. incarnala. - GRANdE CuISSE DE ryMphe. Bot. Cult. no. 3. Très-connue.

230

7 - Var. LE dUC D'Yorck. Lille. C'est une sous-variété de la précédente; fleurs entièrement carnées.

23 I

8 - Var. regia carnea. - Petite cuISsE DE NYMPHE. Bot. cult. $n^{\circ} .4$.

Moins commune que le $N^{\circ}$. 6 .

232

9 -Var.albamedia. - Jennne D'Arc. Vibert. - Alba centifolia. Trianon.

Fleurs régulières, bassinées, moyennes.

10 - Var. alba gracilis. Noisette. $A$ étudier; rameaux très-minces.

1 - Var. nova celestis. - La celeste. Fleurs doubles d'un blanc azuré très-éclatant. 
12 - Var. purpurascens. N.LLA BELLE AURURE. Lille. - Ex albo rosea. Vibert.

Fleurs peu nombreuses, semi-doubles, légèrement pourprées ; feuilles glauques.

13 - Var. Elisa. Godefroy. Trianon.

Plus double que la précédente et moins pourprée.

14 - Var. cannabina. - Blanche A F. DE CHANvre. Godefroy. Trianon.

Monstruosité. Fleurs petites, doubles, d'un blanc mat.

I5 - Var. virens. N. - Blanche a coeur vert. Hort.

F. vertes dans le centre; ouvre rarement bien.

16 - Var. camellia. Fl. de Sèvres.

Fleurs blanches, régulières, ont quelques rapports de forme avec celles du camellia alba.

17 - Var. hybride. - La Senonvinle. Au Luxembourg. Charpentier.

Tient de l'alba et de la suivante; fleurs d'un carné jaunâtre, semi-doubles.

241 xul1. IR. evratina. Bosc.-R. D'ÉRRAT. Trianon.

Le type de ce rosier a été perdu dans la destructiou de l'école.

242

2 - Var. plena. - R. D’Évrat à fl. doubles. Muscade rouge des Holl.

Corymbes très-nombreux; toutes les fleurs n'ouvrent pas.

3 - Var. brevispina. N. -R. A ÉPINES LOURTES. - R. rubispina. Noisette.

N'est pas le rubispina de bosc, lequel même se rapporte à une autre espèce américaine. Ce rosier a fleuri chez moi cette année. Fleurs moyennes, rose vif.

$2 / 4$ xuIv. I R. hibernica. - R. D'IRLANDE. Somm. p. 3x. H. Sabine. Hort.

A fleuri cette année. 
8. Tribu. Roses rouillées. Vulg. églantiers odorans.

Aiguillons inégaux, sétiformes et rarement nuls. Folioles ovales ou oblongues, glanduleuses ( en dessous), dentelures divergentes. Sépales persistantes. Disque épais. Rejetons courbés (à la base).

245 xLv. I R. Lutea. W. - Eglantera. Lin. - R. JAUNE. Mérat par. à Andresy.

M. Parmentier d'Enghien dit le posséder à fleurs doubles.

2 - Var.flore pallido. - R. a fleurs pales. Trianon, potager du roi.

Souchet l'a obtenu de graines du bicolor.

3 - Var. BICOLOR. - R. PUNICEA. - R. CAPUCINE. Autriche. Jacq.

Odeur fétide des fleurs; les fcuilles sentent l'ananas.

248 XLVI. I R. rubiginosa. - R. EGLANTIER ODORANT. Sweet briar des Anglais.

Environs de Versailles; feuilles très-glanduleuses; fleurs moyennes.

2 - Var. a fledrs blanches semi-doubles. $\Lambda$ Chambourcy; il est rare.

3 - Var. a grandes fl. simples. Noisette. Var. D. Somm. p. 33.

La plante de Noisette vient peut-être de celle de Deeandolle.

4 - Var. variegala. - Clénentine de Descenet. - R. J $\mathrm{J}_{\mathrm{Y}}$ de Vibert.

Fleurs grandes, simples, panachées et striées de blanc; bellc variété.

5 - Var. a grandes fleurs semi-doubles roses. Pép. Trianon.

6 - Var. a petites fleuns semi-doubles rose vif. - La petite Hessolse.

Fleurit mieux greflée.

2547 - Var. zabeti de Dupont. H. Thory à Clamart. 
Fleurs petites, plus donbles et plus nombreuses; feuilles moins longues.

8 - Var. ferox. - R. Églantier trés-épineux. Vibert. Noisette. Simple.

Il a fleuri chez moi cette annce. Croissance tardive.

9 - Var. tenuiglandulosa de Mérat, à Yerres. Fruits arrondis comme dans la petite Ilessoise.

ro - Var. muscosa. Églantier odorant mousseux. Angleterre. H. Lodigges.

M. le capitaine Earre m'a procuré cettc variété notable.

I - Var. sepium. - Écrantier odorant des haies.-R. Sepium. Thuillier, Mérat Par. Ce rosier s'éléve à 10 pieds, et s'emploie rarement comme sujet.

$N$. $B$. Je ne cite pas les variétés décrites par Martyn et dessinées par mademoiselle Lawrence; car que je sache, nous n'en possédons allcun.

259 xivu. I R. pulverulenta. Somm. p. 34. - R. PULVÉRULENT. Montagnes du Caucase. Liudley.

Ce rosier est cultivé dans le jardin de M. Sabine, et dans le mien.

260 xLvil. I R. glutinosa.-R. VISQUEUSE. - R. cretica. Somm. p. 35.

An Parnasse, à Candie; ses fruits sont hispides.

261 xuIx. I R. Montezumce. - R. de MONTÉZUME. Somm. p. 35. Nonte-Ventoso près Mexico. Ce rosier se trouve chez M. Boursault.

9. Tribu. Ros iers de chien. Cynorrhodons.

Aiguillons égaux et crochus. Folioles ovales, privées de glandes. Dentelures conniventes. Sépales caduques. Disque épais, couvrant le centre du réceptacle. Rejetons arqués pour la plupart. 
262 L. I R. caucasia. - R. DU CAUCASE. Somm. p. 36. En Sibérie.

Cultivé chez M. Sabine; fruits très-gros.

263 u. I R. canina. - R. DE CHIEN. - R. ÉGLANTIER. Somm. p. 36.

Le plus commun de tous. Sujet pour greffer les autres.

2 - Var. Fl. Semi-dudbles. P. Godefroy.

265

3 - Var. D. collina. - R. oes coteadx. Poir. Haut. de Sèvres. Mérat.

M. Thory a formé un groupe de cette rariété; il lui associe le R. centifolia.

266

4 - Var. E. dumetorum. - R. DES buissons. Th. Pro. 25o. Fontainebleau.

267

5 - Var. casia. Somm. p. 37. - R. canina villosa. F.

Il ne faut pas confondre cette variété avec le tomentosa de Smith.

$N$. B. Je ne parle pas de la var. C rgyptiaca et de plusieurs autres qui ne soṇt pas à portée des amateurs.

${ }_{2} 68$

ı. I R. rubrifolia. - R. A FEUILLES ROU-

GLATRES. - R. gladque. Somm. p. 38. Alpes, Pyrénées.

Devient très-fort; mais n'a pas encore de rariété double.

269 LH. I R. sericea. - R. SOYEUX. id. p. 38. Gossan-Than, Indes-Orientales.

Herbiers de Banks et Lambert $\epsilon n$ Angleterre.

270 LIv. I R. microphylla. - R. A PETITES FEUILLES, Lindl. Mon. - Roxb. Fl. indica. Se trouve en Chine.

N'est pas encore parvenu en Europe; on le voit dans l'llerbier du Clı. Lambert à Londres.

271 Lv. I R. indica. - R. DES INDES. Somm. p. 38. Var. A indica vulgaris. Dans toutes les pépinières. 
Fleurs carnées; iaussement nommé liengale cramoisi. C’est le Eengale thé.

272

2 - Var. B. odoratissima. - R. 'Т

Mal à propos Bengale thć; fleurs très-grandes, odorantes.

${ }_{2} 7^{3}$ LVI. I R. Noiselliana. - R. NOISETTE. N. R. indica. Var. Redouté. Ros.

Envoyée d'A mérique jar $\mathrm{M}$. Ph. Noisette, mais originaire de l'Inde ou de la Chine.

274

2. - Var. B. a fleurs rouges. Hort. 'I'ermaux.

275

2 I - Var. C. pumila. Somm. p. 39.

${ }_{27} 6$

22 - Var. D. longifolia. - R. DEs INDES à feuilles longues. id. p. 39. En Chine.

Cultivé chez Mur. Noisette, Cels, à Malmaison.

277 LVI. I R. semperflorens, - R. FLEURI. - R. vU Bengale. Somm. p. 39.

Répandu partout à cause de sa constante el perpétuelle floraison.

2 - Var. diversifolia. - R. A F. Diverses. Ventenat. H. R. École.

3 - Var. Gr. Fl. Doubles. Trianon. Fleurs pourpre-violet, pleines; n'est pas la rar 4 li. indica.

4 - Var. longifolia. Malmaison.

$28 \mathrm{r}$

5 - Var. Fl: blanches rosétes. Bengale bianc. H. Cels.

Var. médiocre, n'étant pas constante.

6 - Var. a fl. blayches doubles. - Bengale A BouQuets. Trianon.

Corymbes multillores; très-remarquable.

7 - Var. inermis. - Bengale sans Épines. Noisette.

8 - Var. ROUge a pétales houlés. - La CHIFFONNE. 


\section{8}

Variété médiocre; les nouveaux semis l'ont fait oublier.

285

286

287

288

289

290

$29^{I}$

$29^{2}$

$29^{3}$

294

$29^{5}$

296

97
298

9 - Var. hibiscus. Noisette.

Fleurs pourpres; préférable au $\mathrm{n}^{\circ} .8$.

10 - Var. cruenta. - La sanguine. Belle couleur qui varie. Elle sera rapportée au chimasis. II - Var. lavis. - Bengale mousseline.

12 - Var. cerise Éclatante. Vibert. Grandes fleurs semi-doubles, couleur vive.

13 - Var. veloutéE rouge double. Monza. H. Noisette. Semis de Ml. Villarest.

I 4 - Var. bengale LIE DE vin. Idem. Idem.

15 - Var. a fl. panachées. Monza.

16 - Var. la THisbÉ. Vibert.

Fleurs blanches, doubles, très-nombreuses, un peu carnées.

17 - Var. bengale élégant. Noisette. Corymbes nombreux; fleurs doubles, pourpres.

18 - Var. bengale Ternaux. Idem. 19 - Var. Anemating. D'Angleterre.

20 - Var. criamnagana. Fl. Luxembourg. Est peut-être une espèce.

N. B. Toutes les variétés de semis du bengale ne fleurissent qu'une fois l'année.

LVii. I R. laurenceana. - R. DE LAURENCE. Somm. p. 39 . Chine. H. Noisette.

2 - Var. A FL. doubles. IIlem. Bengale pompon; cst bifíre. 
299 LIX. I R. chinensis.-R. DE LA CHINE N.-R. DE la Gifine d'un rouge foncé. Martyn. Dict. des jard. ap. Miller.

Se trouve dans toutes les pépinières; craint la gclée ; espèce naine.

300

2 - Var. B. A fl. doubles. Rose sanguine.

La couleur est plus ou moins intense. Pépinière Noisette.

3 - Var. C. A FL. TRÈS-DOUbles CRAMoisies. Obtenue de semis à Rennes.

Cultivée à Trianon par M. Barrier.

$\mathrm{X}^{\mathrm{e}}$. TRIBU. - Rosiers à styles soudés. Systylés.

Styles adhérens et ramassés en colonne allongée. - Stipules confluentes.

302 Lx. I R.systyla.Decandolle.-R. A STYLES soudés. France. Angleterre.

2 - Var. B. lanceolata. Irlande meridionale. Drummond.

On la trouve dans les environs de Saint-Germain. en Laye.

3 - Var. C. monsonia. Angleterre. Madame Movso.

Envoyé par M. Sabine sous le nom de R. mon sonice.

305 LXI. I R. arvensis. R. DES CHAMPS.

A Velizy près Versailles; était au Jardin du. Roi.

$3 \circ 6$

2 - Var. montana. Villars. Delph.

Le R. serpens est sarmenteux, et couvre les murs; la variété double de Bauhin est perdue.

3 - Var. hybrida. Env. de Versailles.

4 - Var. carnea. - R. DES CHAMPS A FL. ROSÉEs. Angleterre. Sabine.

Il a fleuri chez moi cette année. 
180

$3 \circ 9$

5 - Var. maxima. R. A GR. FL. BLANCHES semi-doubles. Angleterre. Sabine.

Celui-ci fleurit depuis deux ans dans mon jardin.

310 LXII. I R. D’ABYSSINIE. Somm. p. 41 . Abyssinie. Salt.

Herbiers de lanks et Lambert.

31 LXII. I R. sempervirens, - R. TOUJOURS VERT. Somm. p. 42. Provence.

Résiste aux fortes gelées des environs de Paris.

312

2 - Var. latifolia. - R. A LARGES FeUILLES. Luxembourg.

Belle variété peu connue.

3 - Var. prostrala. - R. Rampant. Montpellier. Decandolle.

3 I 4

4 - Var. bulcarica. - R. DE MAHON, ou R. pumila? Willd.

Plus délicat que le précédent.

315

5 - Var. hybrida. - R. du comté d'Ayr; AYRSHire Rose. Écosse. H. Sabine.

Propre à couvrir des murs; fleurs blanches, nombreuses. Il doit être rapporté à l'aromates.

316 Lxiv. I R. multiflora. - R. MULTIFLORE. Thumberg. Japon. Somm. p. 42.

Fleurs blanches, suivant Thumb; est très-rare.

2 - V. carnea. - R. M. CARNÉ. - R. FLORIDA. Ait. Kew. dans les jardins.

C'est le plus commun ; il est sensible aux fortes gelées.

3 - Var. rubra. - R. I. A FL. HoUges. Angleterre et dans nos jardins.

3 I9 Lxv. I R. Brunonii.- - R. DE BROWN. Nepaul. Indes or. Somm. p. 43.

Ilerbiers de Banks et Lambert.

320 LXVI. I R. moschata. - R. MUSCADE. Somm. P. 
43. Barbarie. France méridionale. Iil. semi-doubles.

Les tiges sont attaquées par les fortes gelées.

2 - Var. A FL. Doubles. Hort.

Veut être greffé pour résister à nos grandes gelées.

3 - Var. a grandes fleurs simples. - R. nivea. Dupont. Hort.

Buisson élevé, rustique, remarquable par sa floraisou.

323 LXVIr. I R. rubifolia. - $\mathrm{K}$. A FEUILES DE RONCE. Lindi. Somm. p. 44.

Je l'ai reçu d'Angleterre; fruits pisi formes.

II $^{\mathrm{e}}$. Tribu. - Rosiers banksiens.

Stipules subulées, en alène ou très-étroites, souvent caduques. - Folioles souvent ternées, éclatantes. - Tiges grimpantes.

324 Lxvir. IR. lavigata. - R. DE MICHAUX. Somm. relle.

p. 45. Georgie, Am. sept.

Herbier de Michaux, Muséum d'histoire natu.

325 LxIX. I R. sinica. - R. TRIFOLIÉ. - R. ternata.

Poir. Somm. p. 45. En Chine. H. Noisette.

Sensible aux froids intenses. V. S. avec le fruit Herbier du Dresnay.

326 Lxx: I R. setigera. - R. SATINÉ. Mich. Am. bor. Somm. p. 46.

Amérique septentrionale. V. S. Hérbier $\mathrm{Mi}$ chaux, Muséum d'histoire naturelle.

327 Lxxi. I.R. hystrix. - R. Somm. p. 46. Près de Kiangsi.

En Chine. Herbiers Banks et Lambert. Lindley.

328 LXxI. , R. macrocarpa. - R. A GROS FRUIT. Somm. p. 46. En Chine, prov. de Canton. Staunton.

V. S. Herbiers Banks et Pictet. 


$$
182
$$

329 LXXII. I li. Bancksie, - R. DE BANKS. Somm. p. Fleurs blanches, moyennes, doubles, odeur de violette; naturalisé chez M. Noisetle.

Total : 73 espèces, 329 variétés et sous-variétés. 


Lindley, John/Monographie du genre rosie

di

|

35185000744779 
